Portland State University

PDXScholar

Winter 3-18-2019

\title{
Hospital-Based Services for Opioid Use Disorder: a Study of Supply-Side Attributes
}

Kelsey Caroline Priest

Portland State University

Follow this and additional works at: https://pdxscholar.library.pdx.edu/open_access_etds

Part of the Health Services Administration Commons, and the Substance Abuse and Addiction Commons

Let us know how access to this document benefits you.

\section{Recommended Citation}

Priest, Kelsey Caroline, "Hospital-Based Services for Opioid Use Disorder: a Study of Supply-Side Attributes" (2019). Dissertations and Theses. Paper 4829.

https://doi.org/10.15760/etd.6705

This Dissertation is brought to you for free and open access. It has been accepted for inclusion in Dissertations and Theses by an authorized administrator of PDXScholar. Please contact us if we can make this document more accessible: pdxscholar@pdx.edu. 
Hospital-Based Services for Opioid Use Disorder:

A Study of Supply-Side Attributes

by

Kelsey Caroline Priest

A dissertation submitted in partial fulfillment of the requirements for the degree of

Doctor of Philosophy

in

Health Systems and Policy

\author{
Dissertation Committee: \\ Dennis McCarty, Chair \\ Sherril B. Gelmon \\ K. John McConnell \\ Stephanie Wahab \\ Neal Wallace
}

Portland State University

2019 
(C) 2019 Kelsey Caroline Priest 


\begin{abstract}
The United States (U.S.) is in the midst of an opioid overdose epidemic. In the U.S., overdose deaths related to opioid exposure are the leading cause of accidental death, yet life-saving treatments, such as methadone or buprenorphine (opioid agonist therapy [OAT]), are underused. OAT underuse is due, in part, to complex regulatory and health services delivery environments. Public health officials and policymakers have focused on expanding OAT access in the community (e.g. office-based buprenorphine treatment, and opioid treatment programs); however, an often-overlooked component of the treatment pathway is the acute care delivery setting, in particular hospitals.

Opioid use disorder (OUD)-related hospitalizations are increasing, and incurring significant costs; care delivered in this setting is likely sub-optimal. This study examined hospital-based services for OUD using a conceptual framework based on an interdisciplinary review of policy, organizational behavior, systems science, economics, and health services delivery scholarship. The study's primary research question was: How do supply-side attributes influence hospital OAT delivery, health outcomes, and health services utilization for persons hospitalized with OUD? Supply-side attributes refer to the contextual elements inside and outside of a hospital that may be associated with hospital OAT delivery performance, such as social structures (e.g., hospital standards of care, societal values) and resources and technologies (e.g., hospital staffing, federal treatment policies).
\end{abstract}


A mixed methods study described, explored, and identified how patients with OUD are cared for in the hospital and the barriers and facilitators to delivering OAT during hospitalization. The sequential mixed methods approach (i.e., qualitative followed by quantitative analyses) included analysis of 17 key informant interviews with addiction medicine physicians from 16 non-federal U.S. hospitals, 25 hospital guidance documents from 10 non-federal U.S. hospitals, and administrative data from 12,407 OUD-related hospital admissions from the Veterans Health Administration (VHA) health system.

The findings from the study's three aims and 16 research sub-questions were integrated to reach seven conclusions: 1) OAT is underused in the hospital; 2) OAT delivery varies within and across hospitals; 3) OAT is used ineffectively; 4) non-OAT modalities are inappropriately used during and after hospitalization; 5) supply-side attributes inside and outside the hospital facilitate and impede hospital OAT delivery; 6) demand-side attributes facilitate and impede hospital OAT delivery; and 7) the hospital is an important service delivery mechanism in the OUD care continuum.

The study's findings could be extrapolated to improve policy and practice by implementing education and health service delivery interventions through regulatory and allocative policy mechanisms focused on physicians, medical trainees, and hospital and health system administrators. Understanding how OAT delivery may be improved within the acute care delivery system is an important element to support efforts to curb the ongoing drug poisoning crisis. 


\section{Dedication \\ To my love Christian Eickelberg}

Thanks for sticking with me. Together anything is possible.

And thanks for making the coffee.

To people who use substances

It is a privilege to learn from you, and to work for and with you. 


\section{Acknowledgements}

The completion of my dissertation reflects a decades long personal and professional journey that is possible because of the belief, support, and encouragement of my family, friends, educators, and mentors. I went into medicine and science to become a neurologist and neuroscientist. I was inspired to act because of my dad's early-onset Parkinson's disease diagnosis. My desire was further fueled by working as a research assistant at Oregon Health \& Science University's (OHSU) Balance Disorders Laboratory with Drs. Fay Horak, Laurie King, Patty Carlson-Kuhta, and Triana Nagel Nelson. During this time, my brother James planted the idea that I should obtain an MPH. In 2011, I followed up on that encouragement and enrolled in the MPH program in Health Management \& Policy at Portland State University (PSU). In this program I learned about the broader societal contributions to health and how health care could be improved through systems thinking. This training catalyzed my interest in continuing to learn about how society more broadly shapes health outcomes.

The first time I considered pursuing a PhD was at the completion of my MPH, in the Spring of 2014. While sitting at an MPH awards ceremony my mom leaned over and whispered in my ear and asked me "Why aren't you getting a PhD?" I was intrigued, but I was hesitant because I was about to start medical school, and I wasn't sure that I wanted to add more schooling on top of what was already going to be a long road. I also didn't think I was competitive enough for acceptance into the dual-degree MD/PhD program at OHSU. Despite these misgivings, I ended up applying, and to my surprise, I 
was accepted. I would have never applied without the encouragement of my mentors and advisors from public health and medicine: Drs. Sherril Gelmon, David Jacoby, Neal Wallace, and Johanna Colgrove. They believed I could do it.

My interest in addiction medicine, drug policy, and recovery is a confluence of the personal and professional. My in-laws, Veva Eickelberg and Dr. Steven Eickelberg, show me what a life centered in recovery looks like, with over 60 years of recovery between the two of them. My pursuit of addiction medicine is further guided by my personal values of service, justice, compassion, and the fire-in-my-belly belief that we can do better for people who are frequently marginalized from society. I have seen what better care looks like through the clinical mentorship of Drs. Honora Englander, Jessica Gregg, Todd Korthuis, and Daniel Morris. With these expert clinicians I have had the privilege to participate in and observe compassionate care for patients with substance use disorders using $21^{\text {st }}$ century approaches. It is the efforts of these clinical champions, and the stories of the patients they serve, that inspired the pursuit of the research questions of this dissertation study.

Beyond inspiration, is dedication. I am truly fortunate to have crossed paths with Dr. Dennis McCarty as he concludes his decades-long commitment to working in academia and public service. My dissertation experience would have undoubtedly been different without his relentless support, advocacy, and belief in me. I am forever grateful for his wisdom, his deep knowledge of drug and addiction treatment policy, his 
tenacious editing skills, and his patience. His steadiness and experience guided this dissertation to completion. Thank you, Dennis.

I am privileged to have had so many mentors along the way. From my alma mater, Willamette University, Drs. Peter Harmer, Stas Stavrianeas, and Michael Lockard, who introduced me to what it means to be a rigorous scientist. The members of my dissertation committee Drs. Sherril Gelmon, John McConnell, Stephanie Wahab, and Neal Wallace have provided invaluable insight throughout the dissertation process. The quantitative portion of my dissertation would not have been possible without the generous support and dedication of Dr. Travis Lovejoy, Sarah Shull, and Aldona Herrndorf from the U.S. Department of Veterans Affairs. Other mentors who have meaningfully contributed to my growth and learning include Drs. David Bangsberg, Curtis Harrod, Valerie King, Allison Leof, Miguel Marino, Christina Nicolaidis, and Marta Mellinger. Thank you.

My friends, thank you for your generosity of spirit, encouragement, and understanding of my absence throughout the dissertation process: Janelle Huston, Bailey Parsons, Kaitlyn Selser, Michelle Way, and Kate Williams. A special thank you to Andrea Jones for generously hosting me in spirit and in person in Vancouver, B.C., to Caroline King for proofing and supporting the submission of my dissertation proposal, and to Linda Weick for reminding me how to knit. My classmates from the MPH program, the doctoral program, and from medical school are a source of inspiration: Sarah Andrea, Bekah Bally, Jordan Byers, Kelly Coates, Chad Johnson, Cally Johnson, Kim 
Lepin, Shauna Petchel, Justine Pope, Mary Frances Ritchie, Dave Toffey, Erin ArmondReid, and Laura Zeigen. And to my friends and colleagues at the Center for Evidencebased Policy, the Institute for Healthcare Improvement, and the Relational Leadership Institute; your support was invaluable. Thank you.

Finally, this project was possible because of the generosity of my research participants and the financial support of the National Institute on Drug Abuse (F30 DA044700), the Greenlick Family Scholarship, and Dr. Lovejoy's VA award (VA HSR\&D, 1IK2HX001516). The views expressed in this document are mine and do not represent the views of the U.S. Department of Veterans Affairs, the U.S. Government, or the National Institutes of Health.

Last but not least is my family. You are my bedrock. Thank you Christian, Ben, Nancy, James, Patrick, Laurel, Sarah, Hannah, Alan, Veva, Steve, Garrett, Rufus, and Bart-I love you. 


\section{Table of Contents}

Ackstract

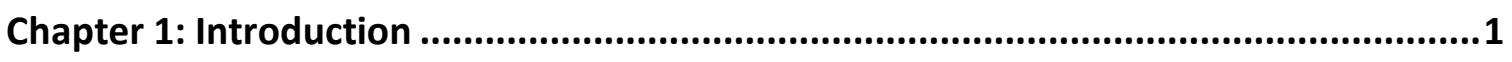

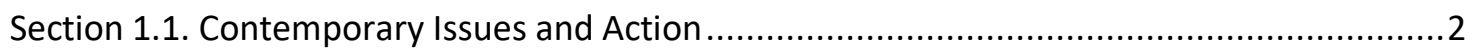

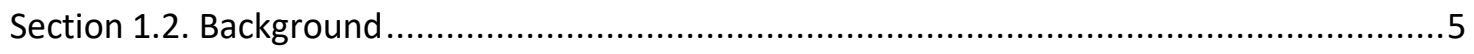

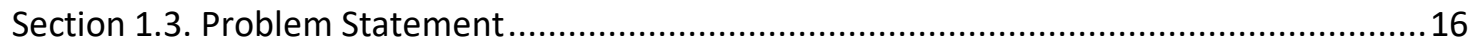

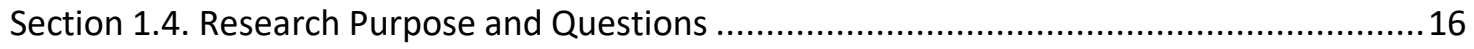

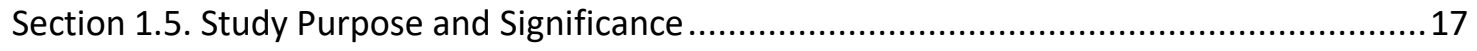

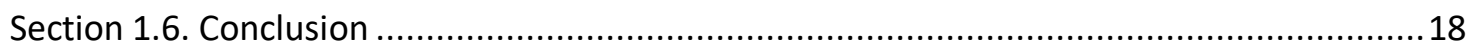

Chapter 2: Literature Review ....................................................................................19

Section 2.1. Defining Drugs, Drug Use Disorders, and Treatment.......................................21

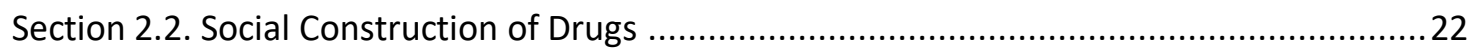

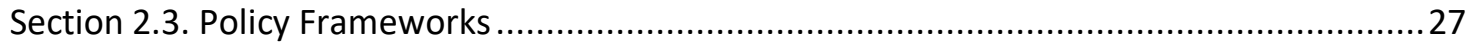

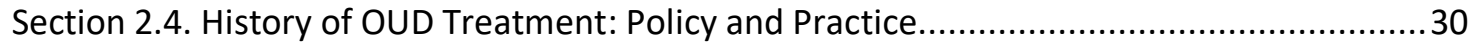

Section 2.5. Contemporary Drug Policy: Regimes, Law, and Regulations............................. 41

Section 2.6. Organizational Behavior Scholarship ............................................................ 51

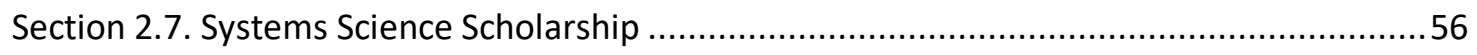

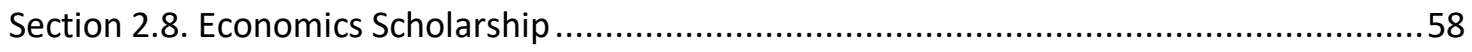

Section 2.9. The U.S. Health Services Delivery Market .......................................................62

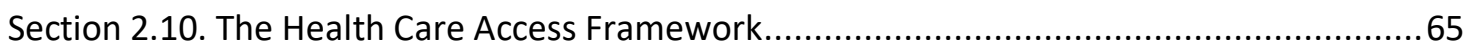

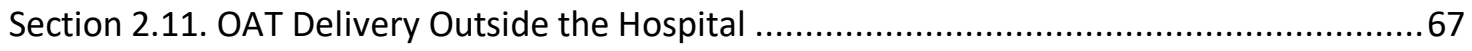

Section 2.12. OAT Delivery within an Integrated Health System ......................................69

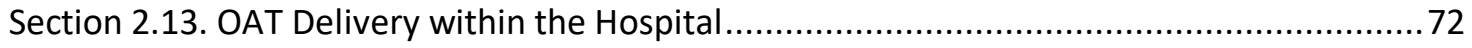

Section 2.14. Development of the Conceptual Framework: Synthesis of the Literature .........94

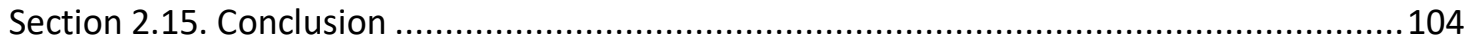

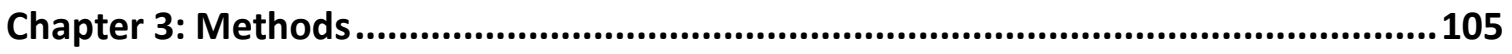




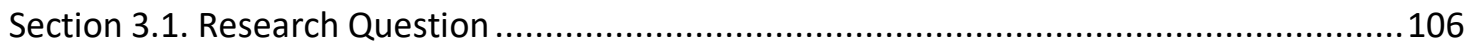

Section 3.2. Study Design, Specific Aims, and Research Sub-Questions .............................107

Section 3.3. Research Approach Justification and Study Assumptions..............................110

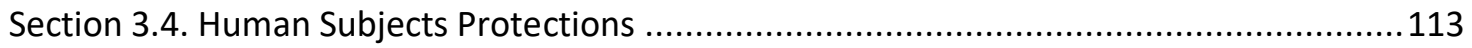

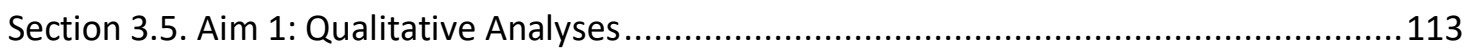

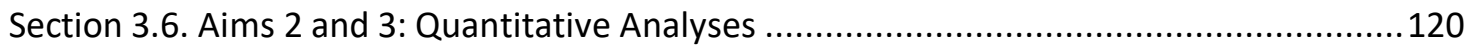

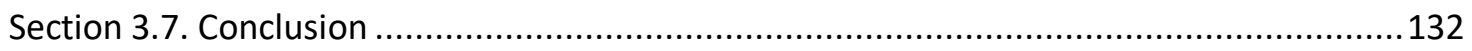

Chapter 4: Presentation and Analysis of Qualitative Data...................................... 133

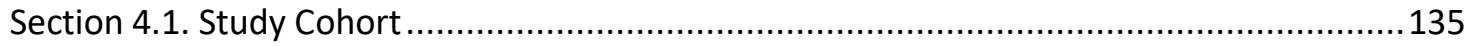

Section 4.2. AMC Service Design and Operations.......................................................... 136

Section 4.3. Facilitators for AMC Service Implementation............................................. 145

Section 4.4. AMC Service Implementation Barriers....................................................... 157

Section 4.5. The Contribution of Demand-Side Attributes ............................................... 170

Section 4.6. Beyond the AMC Service: Emergent Supply-Side Elements.............................171

Section 4.7. Hospital Guidance Documents and Standards of Care ...................................174

Section 4.8. Linking Qualitative Findings to Quantitative Approach ................................. 202

Chapter 5: Presentation and Analysis of Quantitative Data ......................................205

Section 5.1. Patient, Admission, and Hospital Descriptive Statistics ...................................206

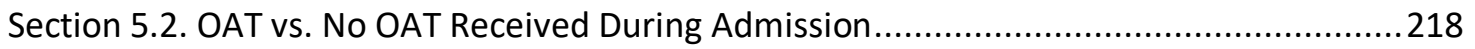

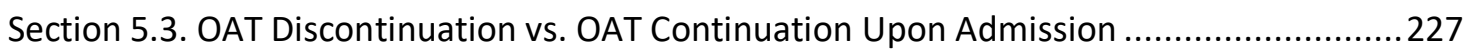

Section 5.4. Hospital OAT Delivery Distribution and Performance Quartiles .......................235

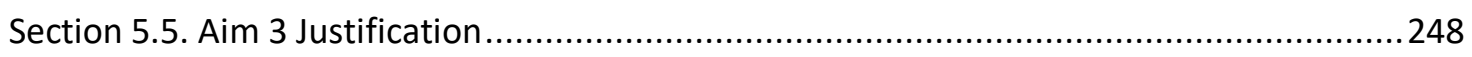

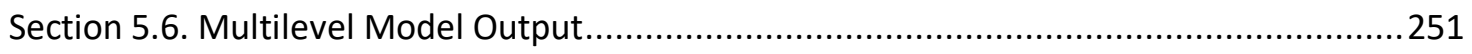

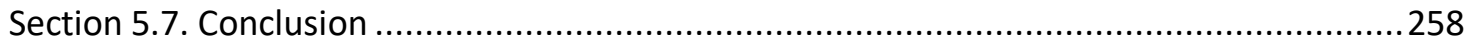

Chapter 6. Discussion, Health System and Policy Implications, and Conclusions ....... 259

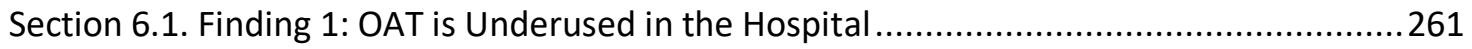

Section 6.2. Finding 2: OAT Delivery Varies Across Hospitals and Health Systems ...............263

Section 6.3. Finding 3: Less Effective OAT Approaches Are Used.....................................265

Section 6.4. Finding 4: Non-OAT Pharmacotherapies are Used .......................................267

Section 6.5. Finding 5: Contextual Hospital Attributes Inform OUD-Related Services ........... 269

Section 6.6. Finding 6: Patient Attributes Facilitate or Deter Hospital OAT Delivery .............274 
Section 6.7. Finding 7: The Hospital has an Important Role in the OUD Care Continuum .....277

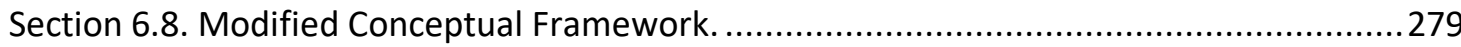

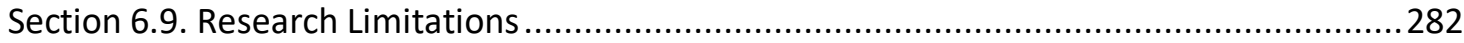

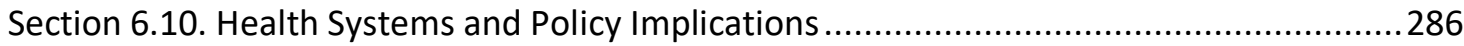

Section 6.11. Future Research Questions and Areas for Exploration ................................298

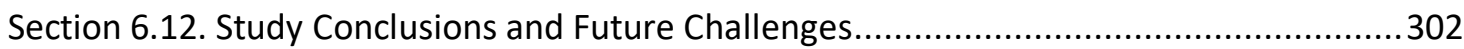

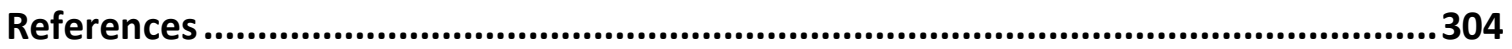

Appendix A: The DSM 5 Opioid Use Disorder Diagnostic Criteria ...............................329

Appendix B: OHSU IRB Approved Informational Sheet.......................................... 331

Appendix C: OHSU IRB Approved Email Recruitment Script ...................................333

Appendix D: OHSU IRB Approved Key Informant Demographic Survey ..................... 335

Appendix E: OHSU IRB Approved Semi-Structured Interview Guide .......................... 337

Appendix F: Final Qualitative Analyses Codebook \& Code Distribution ..................... 341

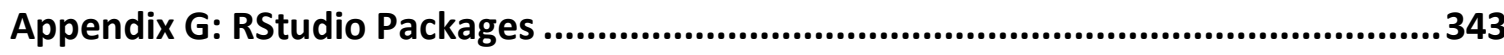

Appendix H: OUD Diagnosis Codes Queried .............................................................344

Appendix I: VHA Study Cohort Consort Diagram ...................................................... 347

Appendix J: Patient and Hospital Study Cohort Definitions........................................348

Appendix K: Patient and Admission Definitions .....................................................350

Appendix L: Co-Occurring Mental Health and Substance Use Disorder Diagnosis Codes

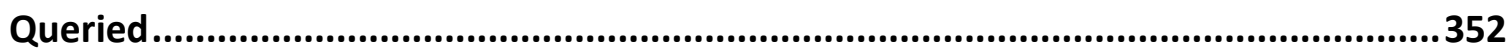

Appendix M: OUD-Related Infection Codes Queried ..................................................373

Appendix N: Non-OAT Pharmacotherapy Definitions............................................. 374

Appendix 0: OAT Pharmacotherapy Definitions ....................................................... 377

Appendix P: Patient Health \& Utilization Definitions ............................................. 378

Appendix Q: Hospital Characteristic Definitions .........................................................379

Appendix R: Sensitivity Analysis: OAT Definition .................................................380 


\section{List of Tables}

Table 2.1. Summary of Recommendations for Hospitalized Patients with OUD..............83

Table 2.2. Literature Reviewed......................................................................................95

Table 3.1. Primary Research Question Operationalized...................................................107

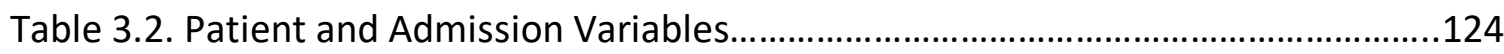

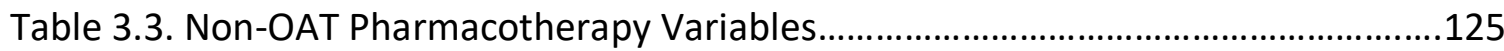

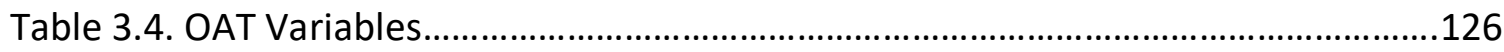

Table 3.5. VHA Patient Health and Utilization Variables.................................................127

Table 3.6. VHA Hospital Characteristic Variables.............................................................127

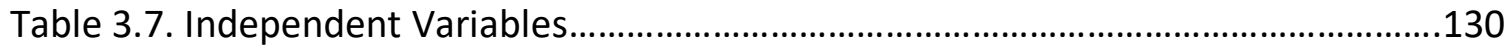

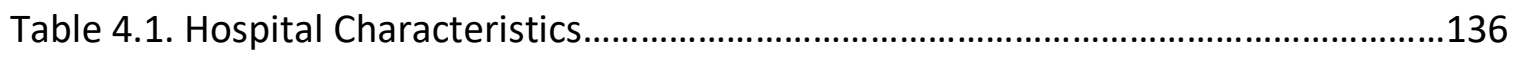

Table 4.2. Addiction Medicine Consult Service Design...................................................139

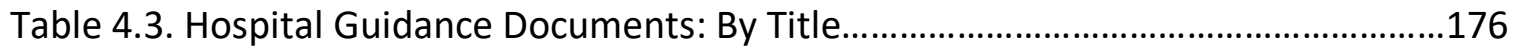

Table 4.4. Hospital Guidance Documents: Content Areas..............................................176

Table 4.5. Guidance Document Recommendations: Pain Management..........................180

Table 4.6. Guidance Document Recommendations: Buprenorphine Continuation........184

Table 4.7. Guidance Document Recommendations: Methadone Continuation...............185

Table 4.8. Guidance Document Recommendations: Withdrawal Management.............187

Table 4.9. Guidance Document Recommendations: OAT Initiation..................................190

Table 4.10. Guidance Document Recommendations: Aberrant Drug Use.......................193

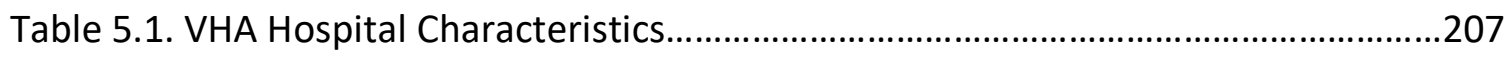

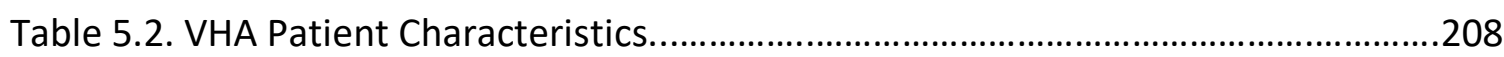

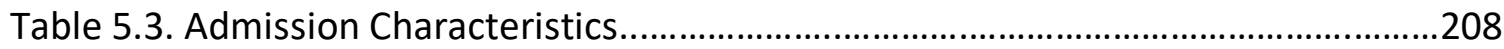

Table 5.4. Health-Related Characteristics During Admission...........................................210

Table 5.5. Health-Related Characteristics: Present on Admission. ..................................211

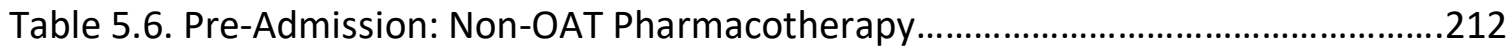

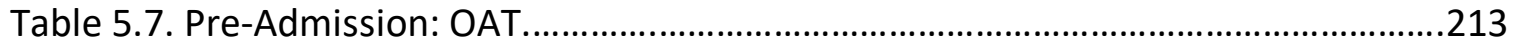

Table 5.8. Admission: Non-OAT Pharmacotherapy. .......................................................214

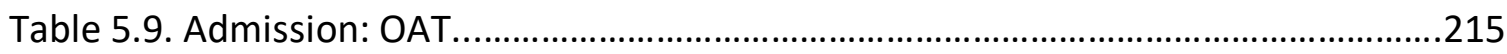

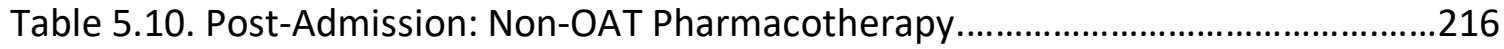

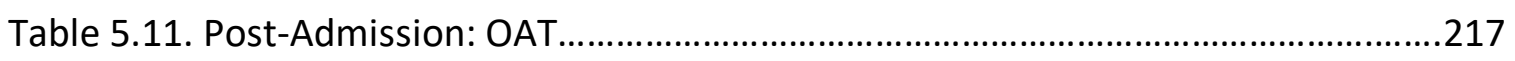

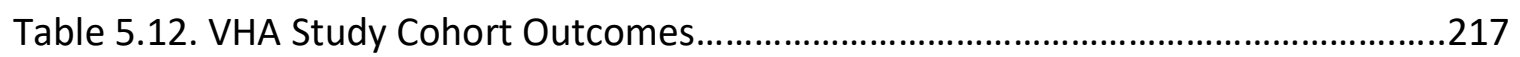

Table 5.13. Patient Characteristics: OAT Received vs. Not Received................................220

Table 5.14. Admission-Related Characteristics: OAT Received vs. Not Received............222

Table 5.15. Admission Non-OAT Pharmacotherapy: OAT Received vs. Not Received....223

Table 5.16. Outcomes: OAT Received vs. Not Received..................................................225 
Table 5.17. Patient Characteristics: OAT Continued vs. Discontinued.

Table 5.18. Admission-Related Patient Characteristics: OAT Continued vs.

Discontinued.

Table 5.19. Admission Non-OAT Pharmacotherapy: OAT Continued vs. Discontinued..232

Table 5.20. Outcomes: OAT Continued vs. Discontinued...................................................233

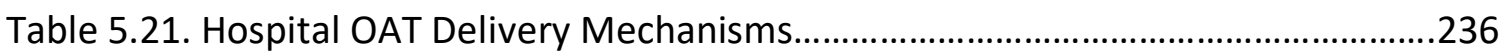

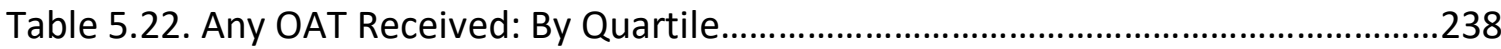

Table 5.23. OAT Delivery Scenarios by VHA Hospital OAT Delivery Performance

Quartile

Table 5.24. Hospital Characteristics by VHA Hospital OAT Delivery Performance

Quartile

Table 5.25. Non-OAT Pharmacotherapy Delivery by VHA Hospital OAT Delivery

Performance Quartile...... 245

Table 5.26. Outcomes by VHA Hospital OAT Delivery Performance Quartile 247

Table 5.27. Testing for Variation in Hospital OAT Delivery Across the VHA 252 Table 5.28. Logistic Regression Models Testing for Multilevel Characteristic Associations with Hospital OAT Delivery..... 255

Table 6.1. Interventions to Enhance OUD Services During Hospitalization. 295

Table 6.2. Informant Suggested Policies for Reform 296 


\section{List of Figures}

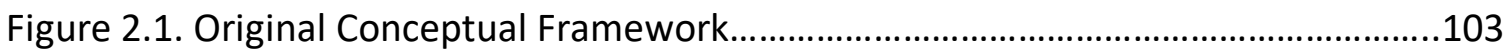

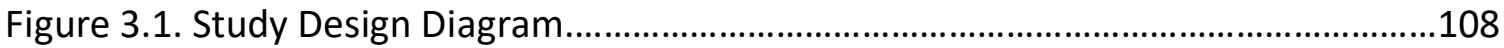

Figure 3.2. Multilevel Modeling Equations.......................................................................131

Figure 5.1. Relative OAT Frequency for Each Hospital by Quartile.................................237

Figure 5.2. Relative OAT Frequency Distribution by Hospital.........................................239

Figure 6.1. Updated Conceptual Framework.....................................................................281 


\section{Glossary}

\begin{tabular}{|c|c|}
\hline Term & Acronym \\
\hline Accreditation Council for Graduate Medical Education & ACGME \\
\hline Akaike Information Criteria & AIC \\
\hline American Psychiatric Association & APA \\
\hline American Society of Addiction Medicine & ASAM \\
\hline Addiction Research Center & ARC \\
\hline Association of American Medical Colleges & AAMC \\
\hline Bayesian Information Criteria & $\mathrm{BIC}$ \\
\hline Centers for Disease Control and Prevention & CDC \\
\hline Centers for Medicare \& Medicaid Services & CMS \\
\hline Code of Federal Regulations & CFR \\
\hline Consult for Addiction Treatment and Care in Hospitals Program & CATCH \\
\hline Delivery System Reform Incentive Program & DSRIP \\
\hline Diagnostic Related Grouping & DRG \\
\hline Diagnostic Statistical Manual & DSM \\
\hline Drug Enforcement Administration & DEA \\
\hline Food \& Drug Administration & FDA \\
\hline Healthcare Common Procedure Coding System & HCPCS \\
\hline Institutional Review Board & IRB \\
\hline International Classification of Diseases, $10^{\text {th }}$ Revision, Clinical Modification & ICD-10-CM \\
\hline Interquartile Range & IQR \\
\hline Intraclass Correlation Coefficient & ICC \\
\hline Intramuscular & IM \\
\hline Intravenous & IV \\
\hline Investigational New Drug & IND \\
\hline Office of National Drug Control Policy & ONDCP \\
\hline Opioid Agonist Therapy & OAT \\
\hline Opioid Treatment Program & OTP \\
\hline Opioid Use Disorder & OUD \\
\hline Peripherally Inserted Central Catheter & PICC \\
\hline Pharmacy \& Therapeutics Committee & P\&T \\
\hline Prescription Drug Monitoring Program & PDMP \\
\hline Project Support for Hospital Opioid Use Treatment & SHOUT \\
\hline Skilled Nursing Facility & SNF \\
\hline Substance Abuse and Mental Health Services Administration & SAMHSA \\
\hline Substance Use Disorder & SUD \\
\hline United Nations & U.N. \\
\hline United States & U.S. \\
\hline Variance Inflation Factor & VIF \\
\hline Veterans Affairs & VA \\
\hline Veterans Health Administration & VHA \\
\hline World Health Organization & WHO \\
\hline
\end{tabular}




\section{Chapter 1: Introduction}

This dissertation explores the influence of supply-side attributes (e.g., social, financial, and policy-related) contributing to opioid agonist therapy (OAT) delivery for hospitalized patients with opioid use disorder (OUD). This chapter provides a broad overview of the foundational issues related to OAT delivery that guided the rationale and design of this dissertation study, presented in six sections:

1. Section 1.1 introduces the contemporary issues and action related to the opioid overdose epidemic.

2. Section 1.2 provides background for the dissertation including defining and describing opioids, OUD, and OUD treatments.

3. Section 1.3 outlines the study's problem statement.

4. Section 1.4 states the study's research question and three aims.

5. Section 1.5 describes the study's purpose and significance.

6. Section 1.6 articulates this chapter's conclusion.

Following Chapter 1, Chapter 2 provides an interdisciplinary literature review and describes the conceptual framework proposed for the study. Study methods are detailed in Chapter 3 and results are presented in Chapter 4 (qualitative) and Chapter 5 (quantitative). Chapter 6 discusses and integrates study findings to extract seven primary results, details the limitations and assumptions of the research, updates the study's conceptual framework, provides strategies for policy change, and concludes with questions for future research. 


\section{Section 1.1. Contemporary Issues and Action}

The undertreatment and subsequent preventable suffering and deaths of persons with OUD are pressing public health issues. An estimated 33 million people globally use opioids (United Nations [UN] Office on Drugs and Crime, 2016), 2.3 million people in the United States (U.S.) live with an OUD (Center for Behavioral Health Statistics and Quality, 2016), and in 2017, opioids were involved in nearly 48,000 overdose deaths (Centers for Disease Control and Prevention [CDC], 2018). In addition to mortality, OUD incurs total annual costs of $\$ 78.5$ billion related to loss of productivity, crime, and health care (Florence, Zhou, Luo, \& Xu, 2016). The largest contributor to annual costs is health care service delivery (33\%), which starkly contrasts with the costs of providing OUD treatment (4\%) (Florence et al., 2016).

OUD related hospitalizations likely drive health care costs for persons with OUD. Between 2005 and 2014 the national rate of OUD-related hospitalizations increased 64\% (Weiss et al., 2016). As compared with hospitalizations for other conditions, persons with OUD were admitted for almost a day longer ( 4.5 days vs. 5.2 days) and had nearly triple the costs $(\$ 10,400$ vs. $\$ 28,543)$ (Ronan \& Herzig, 2016; Weiss \& Elixhauser, 2014). Moreover, for persons with OUD with a concurrent drug-use related infection (e.g., endocarditis, osteomyelitis), the length of stay more than tripled (14.6 days) and the costs rose to $\$ 107,217$ (Ronan \& Herzig, 2016). Hospitalization costs for persons with OUD are estimated at $\$ 15$ billion annually, the majority of which are associated with publicly insured and uninsured individuals (Ronan \& Herzig, 2016). 
The loss of life and the financial costs attributed to OUD may be mitigated with treatment, specifically using OAT: methadone and buprenorphine. The Food \& Drug Administration (FDA) approved methadone for OUD treatment in 1972 (Institute of Medicine Committee on Federal Regulation of Methadone, 1995) and buprenorphine in 2002 (FDA, 2002). Despite decades of FDA approval and years of robust research (Mattick, Breen, Kimber, \& Davoli, 2009, 2014; Nielsen et al., 2016), the number of treatment facilities offering OAT is lower than the growing need. Only $34 \%$ of substance use disorder (SUD) treatment facilities in the U.S. offer OAT or antagonist therapy (i.e., naltrexone) (Knudsen, Abraham, \& Roman, 2011) and only $10 \%$ of patients in these clinical settings received OUD pharmacotherapy (Knudsen \& Roman, 2012).

Explanations for OAT underuse are well described in outpatient and addiction treatment facility settings, and are attributed to financial, regulatory, geographic, and health care professional attitudinal factors (Sharma, Lamba, Cauderella, Guimond, \& Bayoumi, 2017). Systemic and institutionalized structural and sociologic elements (e.g., racism, criminal justice status, insurance benefits) further exacerbate OAT underuse (Cummings, Wen, \& Ko, 2016; Grogan et al., 2016; Merrall et al., 2010; Saloner, Bandara, McGinty, \& Barry, 2016; Saloner \& Cook, 2013).

The nationwide opioid overdose crisis has sparked the attention, action, and collaboration of policymakers in federal and state government offices, legislative bodies, and agencies. In the past decade, legislation has shaped OUD treatment practices, such as the Comprehensive Addiction Recovery Act of 2016, the $21^{\text {st }}$ Century Cures Act, and 
the 2018 SUPPORT for Patients and Communities Act. During President Barak Obama's administration, the Office of the Surgeon General launched the Turn the Tide RX Campaign (Surgeon General of the United States, n.d.) and published the first Surgeon General's Report on SUDs (U.S. Department of Health \& Human Services, 2016). President Donald Trump issued an executive order establishing the President's Commission on Combating Drug Addiction and the Opioid Crisis recommending actions to address the opioid crisis (White House, 2017). The policy efforts and awareness campaigns contributed to the Presidential declaration of the opioid crisis as a federal public health emergency on October 26, 2017 (Substance Abuse and Mental Health Services Administration [SAMHSA], 2017a).

Federal agencies (e.g., Centers for Medicare \& Medicaid Services (CMS) and SAMHSA) and state Governor's offices have supported initiatives to address the opioid overdose epidemic. In 2015 and 2016, CMS provided opportunities for state Medicaid agencies to redesign their SUD delivery systems to expand access to treatment using $\S 1115$ waivers, and updated the Medicaid managed care rules to allow for treatment in Institutions for Mental Diseases (Priest, Leof, McCarty, \& King, 2017). SAMHSA's Center for Substance Abuse Treatment and Center for Substance Abuse Prevention provided additional treatment funding opportunities for single state agencies through the State Targeted Response to the Opioid Crisis Grants program (SAMHSA, 2017b). An assessment of program funding allocation observed that $71 \%$ of grantees used the awards to expand OAT capacity (Beletsky, 2018). Other state-level interventions 
included declarations of emergency by the Governor's Offices of Alaska, Arizona, Florida, Maryland, Massachusetts, and Virginia, which allowed for increased access to state funds and resources to expand addiction services and to develop prescribing guidelines (Baker-White, 2017).

Although policymakers are acting, many public health officials and clinical leaders are calling urgently for a more robust national public health strategy to decrease mortality and morbidity associated with the opioid overdose epidemic (Saloner et al., 2018). In 2018, experts identified seven key priorities for U.S. policymakers: 1) improved data collection; 2) safer opioid prescribing; 3) stigma reduction; 4) harm reduction; 5) criminal justice reform; 6) regulatory changes; and 7) treatment expansion (Saloner et al., 2018). Treatment expansion, in the hospital setting, is the focus of this dissertation.

\section{Section 1.2. Background}

The U.S. has a long and complex history with opioids, OUD, and OUD treatment. Health professionals and society more broadly may simultaneously view opioids as legal medicine for patients and as an illegal drug used by criminals. This dissertation considers the existence of these different perspectives, the influence of these perspectives on system design, and the institutionalization of these perspectives through policy.

1.2.1. Opioids. Throughout history, healers and medical professionals used opioids to decrease pain (Benyamin et al., 2008). The term opioid is attributed to Professor George Acheson, who was the first to use this nomenclature to categorize compounds with morphine-like pharmacological and agonist effects (Martin, 1983). 
Opioids are produced endogenously, within the body, and may be ingested exogenously from sources external to the body (Feng et al., 2012). Exogenous opioids may be environmentally derived (e.g., opium from the plant Papaver Somniferum) (Hurley et al., 2007) or synthesized through pharmaceutical manufacturing (e.g., hydrocodone). Upon ingestion of opioids-oral, intravenous, inhalation, nasal insufflation-the compound binds to mu, kappa, and delta opioid receptors located predominantly in the limbic areas of the brain (Hurley et al., 2007). Opioid receptors are involved in a variety of physiologic processes such as, but not limited to: pain modulation; emotional response; feeding; the regulation of membrane ionic homeostasis; cell proliferation; immune function; respiratory and cardiovascular control; and pathophysiological processes (e.g., epileptic seizures, obesity, addiction) (Feng et al., 2012).

Individuals may obtain opioids through legal and illegal markets. The allowable possession and manufacturing of opioids is statutorily defined by the Controlled Substances Act of 1970 and monitored by the Drug Enforcement Administration (DEA). In 1970, Congress established a scheduling system to categorize legal and illegal opioids. Illegal opioids, Schedule I substances, such as diacetylmorphine (also known as heroin), are deemed by the U.S. federal government to not have a currently accepted medical use (DEA, n.d.). The government categorizes legal FDA-approved opioids (e.g., morphine) as Schedule II, III, IV, or V (DEA, n.d.). These opioids have medically accepted uses and are manufactured through legal manufacturing processes, but their use is illegal, in most circumstances, when used for non-FDA approved indications. 
The U.S. is the world's largest consumer of opioids (University of Wisconsin, 2017). Between 1999 and 2012 the number of U.S. opioid prescriptions tripled, and in 2012 opioid prescription expenditures across all payers, including self-insured, totaled $\$ 7.4$ billion dollars (Zhou, Florence, \& Dowell, 2016). Opioid overconsumption is not limited to the last 25 years. The current opioid overdose epidemic follows a succession of past epidemics, such as the morphine crisis post-civil war in the 1870s (Courtwright, 2015), the youth heroin epidemic of the 1950s (Campbell, Olsen, \& Walden, 2008), and the Vietnam war veteran heroin emergency of the 1970s (Schmidt, 1971).

It is also important to recognize the changes in the contemporary opioid supply, which has been coined the "triple epidemic" and features a "rising waves of deaths due to separate types of opioids each building on top of the prior wave" (Ciccarone, 2017, p. 107). The first wave of overdose deaths in the 1990 s was predominantly from prescription opioids (Ciccarone, 2017). The second wave, starting in 2010, was due to heroin (Warner, Trinidad, Bastian, Miniño, \& Hedegaard, 2016), and the third and current wave is attributed to the growing supply of synthetic opioids, including illicitly manufactured fentanyl and fentanyl analogues (R. G. Frank \& Pollack, 2017; Gladden, Martinez, \& Seth, 2016; Warner et al., 2016). The shift in the illegal drug supply from heroin to fentanyl has already happened in parts of Canada. In Vancouver, British Columbia, recent data reflects that $90 \%$ of presumed heroin tested positive for fentanyl (Tupper, McCrae, Garber, Lysyshyn, \& Wood, 2018). The shift in the Vancouver supply is a harbinger of changes to the U.S. opioid drug supply. 
1.2.2. Opioid use disorder. Health experts are concerned with the excessive use of opioids because of the association with increased morbidity and mortality, in particular for men and early onset users (Cottler et al., 2016). When taken in excess, opioids can cause central nervous system depression leading to respiratory failure and death (Bollinger et al., 2012), shortened life expectancy (Hayes et al., 2011), and increase the risk of addiction (Compton \& Volkow, 2006). In 2016, the CDC published recommendations on the safety of consuming opioids for the treatment of chronic noncancer pain (Dowell, Haegerich, \& Chou, 2016). The CDC prescription opioid guidelines specified that prescribers should avoid prescription opioids for chronic non-cancer pain and stated that opioid consumption is not safe at any dose (Dowell et al., 2016). In 2017, the National Academy of Sciences published Pain Management and the Opioid Epidemic: Balancing Societal and Individual Benefits and Risks Of Prescription Opioid Use and recommended that the FDA and other organizations take action to reduce OUDrelated harms (Committee on Pain Management and Regulatory Strategies to Address Prescription Opioid Abuse, 2017).

For the purpose of this dissertation OUD is conceptualized as a brain disease that is treated through the medical model. The diagnosis of opioid addiction follows the criteria from the Diagnostic and Statistical Manual of Mental Disorders, Fifth Edition (American Psychiatric Association [APA], 2013). The diagnosis criteria characterize OUD as a problematic pattern of opioid use that leads to clinically significant impairment or distress occurring within a 12-month period (APA, 2013). The diagnostic criteria for OUD 
states that a patient must have at least two of 11 symptoms, and that the severity of illness is to be categorized as mild ( 2 to 3 symptoms), moderate ( 4 to 5 symptoms), or severe ( 6 or more symptoms). The symptom criteria are listed in Appendix A (APA, 2013). The DSM also requires clinicians to distinguish among early remission, sustained remission, the use of pharmacotherapy, or if the patient is receiving care in a controlled environment (APA, 2013). Other definitions of addiction do exist. The American Society of Addiction Medicine [ASAM] (2011) defines addiction as:

A primary, chronic disease of brain reward, motivation, memory and related circuitry. Dysfunction in these circuits leads to characteristic biological, psychological, social and spiritual manifestations. This is reflected in an individual pathologically pursuing reward and/or relief by substance use and other behaviors. Addiction is characterized by inability to consistently abstain, impairment in behavioral control, craving, diminished recognition of significant problems with one's behaviors and interpersonal relationships, and a dysfunctional emotional response. Like other chronic diseases, addiction often involves cycles of relapse and remission. Without treatment or engagement in recovery activities, addiction is progressive and can result in disability or premature death.

1.2.3. Treatment. The gold standard treatment for OUD is OAT because it is well studied, safe, and effective (Mattick, Breen, Kimber, \& Davoli, 2003; Mattick et al., 2014). There are three FDA-approved OATs, with multiple formulations: 1) buprenorphine (sublingual tablet, implant, extended-release, injection, and implant); 2) buprenorphine/naloxone (sublingual tablet and buccal film); and 3) methadone (liquid, tablet). The World Health Organization (WHO) lists OAT on their essential medication list (WHO, 2017) and recommends that all national health systems provide widespread OAT access for persons with OUD (WHO, 2009). Unfortunately, OAT access in the U.S. is 
constrained because of special federal regulations that govern where, when, and who may administer or prescribe OAT.

1.2.3a. Opioid treatment programs. Methadone administration for OUD, a Schedule II controlled substance, is governed by the Certification of Opioid Treatment Programs (OTPs), 42 Code of Federal Regulations (CFR) 8 (SAMHSA, 2015). Under these rules, methadone must be administered daily under direct observation in a federallylicensed OTP with two exceptions: 1) during hospitalization; and 2) approved take home-doses (Priest et al., 2019). OTPs may also administer buprenorphine, offer other services such as recovery fellowship (e.g., 12-Step), and require patient participation in non-pharmacotherapy services such as counseling (SAMHSA, 2015).

1.2.3b. Community treatment programs. Currently, the only FDA-approved OAT that may be legally prescribed by federally-certified prescribers is buprenorphine (with and without naloxone), a Schedule III controlled substance (SAMHSA, 2016). To become a certified prescriber, a health professional must meet the requirements of the Drug Addiction Treatment Act of 2000, including: licensed to practice under state law, registered with the DEA, and completed prescriber training (SAMHSA, 2016). A variety of primary care models for buprenorphine delivery have emerged (e.g., office-based opioid treatment programs, the Buprenorphine HIV Evaluation and Support Collaborative Model) (Korthuis et al., 2017).

1.2.3c. Integrated health systems. There is much to learn about the delivery of OAT and OUD services from integrated health systems because of the disjointed nature 
of the U.S. addiction treatment system. The largest integrated health system in the U.S. is the Veterans Health Administration (VHA), which has a long-standing history of providing services for OUD. The VHA is facing a growing OUD patient population (Finlay et al., 2016; Oliva, Trafton, Harris, \& Gordon, 2013) that is twice as likely to die from accidental overdose than non-veterans (Bohnert, Ilgen, Galea, McCarthy, \& Blow, 2011).

The VHA OUD treatment services system began nearly 60 years ago, with the creation of the Alcohol and Drug Dependence Service, to meet the needs of veterans returning from the Vietnam War (Wyse et al., 2018). Since that time, the VHA has implemented policy, educational, and quality improvement initiatives to increase OAT capacity across the system (Wyse et al., 2018). The VHA added OAT, specifically buprenorphine, to the VHA formulary in 2006, and in 2008, mandated the use of OAT as a "minimum clinical requirement" for OUD treatment (Wyse et al., 2018). Further, in 2015, the VHA published system-wide clinical guidance documents on OAT delivery (Wyse et al., 2018). Despite these system-wide initiatives, recent utilization data reports that only $38 \%$ of OUD patients receive pharmacotherapy and that there is significant variation in facility pharmacotherapy performance (3\% to 74\% delivery) (Finlay et al., 2018).

Data from outside the VHA suggests similar or worse OAT delivery performance. In 2016, a national survey of U.S. SUD facilities observed that only $36 \%$ of facilities offered all three types of OUD pharmacotherapy (OAT or opioid antagonist). Additionally, in a study of 154 non-VHA specialty addiction treatment programs, only 
$10 \%$ of patients received some form of OUD pharmacotherapy (Knudsen \& Roman, 2012). These studies suggest there is still a need to improve access to and the delivery of OAT in the community, both inside and outside the VHA. Although warranted and important, VHA research and system-wide initiatives to date have focused on outpatient OAT delivery, and research on hospital OAT delivery has not yet occurred.

1.2.3d. Hospital OAT delivery. There is historic precedent for hospital-based OUD treatment. Similar to contemporary outpatient OAT delivery systems informed by public policy, hospital OAT delivery was born out of necessity. In the mid-1920s, the Supreme Court ruled that it was illegal for physicians to provide opioids to patients with OUD in the outpatient setting (Hohenstein, 2001) and care shifted to the hospital (White, 2002). This care delivery trend lasted for about 10 years, falling out of favor because of the ineffectiveness of the approach (White, 2002). In its place, the federal government created two addiction hospitals called "narcotic farms" managed by the U.S. Public Health Service and the U.S. Bureau of Prisons (White, 2002). The narcotic hospitals, one in Lexington, Kentucky, and the other in Fort Worth, Texas, were the primary source of addiction treatment until the advent of outpatient methadone maintenance therapy in the late 1960s (White, 2002). The addiction hospitals closed in the 1970s (White, 2002).

Nearly 40 years later, there is a renewed interest in hospital-based services for OUD, likely inspired by the ongoing opioid overdose epidemic and the subsequent increased utilization of acute care delivery resources. The following sub-sections provide 
evidence suggesting that hospital-based OUD treatment services are urgently needed, care for this population is feasible and effective, and potential barriers to care in this setting should be explored.

1.2.3d.1. The need. The national rate of OUD-related hospitalizations increased 64\% between 2005 and 2014 (Weiss et al., 2016). Moreover, between 2013 and 2015, opioid-related admissions (217 per 100,000 people) were the second most common SUD admission behind alcohol (558 per 100,000) (Fingar et al., 2018). In 2015, the cumulative costs for OUD-related hospitalizations were estimated at \$15 billion annually (Ronan \& Herzig, 2016). Costs for OUD-related hospitalizations were higher (\$28,543 vs. $\$ 10,400$ days), and stays were longer (5.2 days vs. 4.5 days), in contrast with non-OUD related admissions (Ronan \& Herzig, 2016; Weiss \& Elixhauser, 2014). The distribution of costs by payer for OUD-related hospitalizations disproportionately burdens public payers $-72 \%$ of admissions were paid for by Medicaid (40\%) and Medicare (32\%) (Weiss \& Heslin, 2018).

Persons with OUD-related hospital admissions have a relatively low rate of hospital death, although the in-hospital mortality rates are increasing for patients who receive higher levels of care during admission. In 2012, 1\% of OUD-related hospital admission patients and 3\% of OUD-related co-infection admissions patients died (Ronan \& Herzig, 2016). There was a significant increase in hospital deaths in the ICU for opioid overdose between 2009 (mean mortality rate 7\%) and 2015 (mean mortality rate 10\%) 
(Stevens et al., 2017). However, little is known about OUD-related health outcomes that occur after discharge, a potentially vulnerable time for overdose.

A limited literature base characterizes the sub-optimal and potentially inappropriate care received during and after hospital admission for patients with OUD. Sub-optimal care for OUD may be reflected in three ways: 1) elevated rates of leaving the hospital against medical advice (Ronan \& Herzig, 2016; Stranges, Wier, Merrill, \& Steiner, 2009); 2) low OAT use during admission (Rosenthal, Karchmer, Theisen-Toupal, Castillo, \& Rowley, 2015); and 3) low OAT use in the 30 days after hospital discharge (Frazier et al., 2017; Naeger, Ali, Mutter, Mark, \& Hughey, 2016). Inappropriate care may be further reflected in the increased use of short-acting opioids during (Herzig et al., 2014) and after admission (Larochelle, Liebschutz, Zhang, Ross-Degnan, \& Wharam, 2016; Naeger, Mutter, Ali, Mark, \& Hughey, 2016).

\subsection{3d.2. The solution. A growing evidence base supports the use of} interventions to enhance services for patients hospitalized with OUD, including: a clinical trial on OAT delivery (Liebschutz et al., 2014); several hospital practice checklists and recommendations based on narrative reviews (Noska, Mohan, Wakeman, Rich, \& Boutwell, 2015; Sharma et al., 2017; Thakarar, Weinstein, \& Walley, 2016; TheisenToupal, Ronan, Moore, \& Rosenthal, 2017; Weinstein, Wakeman, \& Nolan, 2018); federal guidance documents (SAMHSA, 2018; Institute of Medicine Committee on Federal Regulation of Methadone, 1995); and the retrospective and prospective 
evaluations of addiction medicine consult (AMC) services (Nordeck et al., 2018; Suzuki, 2016; Trowbridge et al., 2017; Wakeman, Metlay, Chang, Herman, \& Rigotti, 2017).

1.2.3d.3. The barriers. A limited literature base describes how supply-side attributes, inside and outside the hospital, may contribute to hospital OAT underuse. Potential barriers outside the hospital include treatment coverage policies imposed by third-party payers (Hassamal et al., 2017) and broader federal treatment regulations (Hassamal et al., 2017). Not explored explicitly in the literature is the potential misinterpretation of specific federal regulations that dictate practice for opioid withdrawal in the hospital setting (i.e., 21 CFR 1306.07, sections b and c) (Nagel, 2002). Within the hospital, potential barriers to OAT delivery are likely connected to a number of factors, including: 1) provider knowledge deficits related to OAT and OUD treatment; 2 ) the perpetuation of stigmatizing behavior by providers towards patients with OUD because of knowledge deficits; 3 ) concerns about external federal regulations (e.g. DEA audits); 4) concerns about outside perceptions (e.g., worried about attracting OUD patients); 5) a lack of support staff and institutional support; 6) frequent laboratory testing; and 7) an inadequate referral network (Hassamal et al., 2017). Another potential barrier is whether the hospital has the correct staff employed. Patients with OUD and other SUDs, for example, have expressed interest in receiving services from peer support providers (i.e., professional with lived experience) during hospitalization (Velez, Nicolaidis, Korthuis, \& Englander, 2017). Despite this, peer support providers are a group of professionals not ususally employed by hospitals. 


\section{Section 1.3. Problem Statement}

Limited information exists describing hospital OAT care delivery patterns and why hospital OAT is likely underused. This dissertation seeks to enhance the understanding of supply-side attributes on hospital OAT delivery by considering the broader contextual elements that shape practice through an interdisciplinary research lens grounded in theories, models, and frameworks from policy theory (Ingram \& Schneider, 1990, 1991; MacCoun, Saiger, Kahan, \& Reuter, 1993; Pierson, 1993; A. L. Schneider \& Ingram, 1988, 1993; Skocpol, 1992), organizational behavior (DiMaggio \& Powell, 1983, 1991; Katz \& Kahn, 1978; Leavitt, 1962; Scott, 2003; Scott \& Meyer, 1991; Thompson, 1967), systems science (Basole \& Rouse, 2008; Ferlie \& Shortell, 2001; Lipsitz, 2012; Perrow, 1986; Plsek \& Greenhalgh, 2001; Sallis, Owen, \& Fisher, 2015), economic theory (Alchian \& Demsetz, 1972; Allison \& Zelikow, 1999; Eisenhardt, 1989; Friedman, 2002; Jensen \& Meckling, 1976; Simon, 1985; Williamson, 1979, 1985, 1994; Williamson \& Ouchi, 1981; Zeckhauser \& Pratt, 1985), and health services delivery (Levesque, Harris, \& Russell, 2013).

\section{Section 1.4. Research Purpose and Questions}

An integrated mixed methods design (i.e., qualitative followed by quantitative), comprised of three aims and 16 research sub-questions, explored this broad research question: How do supply-side attributes influence hospital OAT delivery, health outcomes, and health services utilization for persons hospitalized with OUD? The data for Aim 1, the qualitative aim, were 17 key informant interviews from 16 non-VHA U.S. hospitals, and 25 non-VHA hospital guidance documents. The data for Aims 2 and 3, the 
quantitative aims, were deidentified administrative data from a retrospective national sample of patients with OUD from the VHA health system in the fiscal year of 2017. Aim 1 examined how addiction medicine experts and their hospitals delivered OAT for patients with OUD and Aims 2 and 3 described current OAT delivery practices in VHA hospitals:

- Aim 1: Examine the influence of supply-side attributes inside and outside of nonVHA hospitals that shape hospital OAT delivery;

- Aim 2: Describe and test variation in VHA hospital OAT delivery;

- Aim 3: Test associations among patient and hospital characteristics on VHA hospital OAT delivery.

\section{Section 1.5. Study Purpose and Significance}

This study sought to describe and understand the patterns and determinants of evidence-based hospital OAT delivery, a first step towards ensuring that hospitalized patients with OUD receive effective treatments, and that treatment policies are crafted and systems are designed to support the delivery of these modalities. This study has the potential to contribute to health services delivery improvement because it explores the potential contributory contextual elements (e.g., policy, organization, system, and economic factors) and analyzes administrative data from an integrated health system to describe current practice. This study addresses public health, clinical practice, and health system delivery knowledge gaps by connecting the research domains of public health, health systems, health policy, and health services delivery. 


\section{Section 1.6. Conclusion}

Hospital OAT underuse engages issues of care quality, safety, and cost.

Understanding the barriers and facilitators to hospital OAT delivery is a first step

towards informing interventions inside and outside the hospital to ensure that OUD

hospitalized patients receive effective treatments. Obtaining a greater understanding of

where and how OAT delivery may be improved across the health services delivery

system is especially important due to the current opioid overdose epidemic. 


\section{Chapter 2: Literature Review}

The ongoing opioid overdose epidemic has increased the number of persons in the United States (U.S.) with opioid use disorder (OUD) and OUD-related hospitalizations. A limited literature base describes hospital OAT underuse and nearly no literature exists exploring why OAT is underused in the inpatient setting. OAT is a wellstudied life-saving treatment for persons with OUD. Hospitals may play an important role in the contemporary OUD treatment pathway. Policy, regulatory, and system-level factors likely complicate hospital-based OAT delivery, therefore this dissertation seeks to understand the influence of these attributes on the underuse and variation of hospital OAT delivery. Chapter 2 includes a comprehensive literature review organized into 15 sections:

1. Section 2.1 provides study definitions for drugs, drug use disorders, and OAT.

2. Section 2.2 explains how drug use disorders and drugs are socially constructed and the two frames dominating the narrative in health policy and practice.

3. Section 2.3 outlines policy frameworks (the Social Construction Framework; the Comparative Drug Policy Analytic Framework; and the Policy Feedback Theory) to support the rationale that national, state, and local health care policies influence hospital OAT delivery.

4. Section 2.4 provides a 150 -year historical overview of OUD treatment practice and policy. 
5. Section 2.5 describes the contemporary drug policy environment, including the policy regimes, laws, and regulations informing OAT delivery.

6. Section 2.6 reviews organizational behavior scholarship relevant to understanding hospital OAT delivery.

7. Section 2.7 examines systems science models and theory that may affect hospital OAT delivery.

8. Section 2.8 discusses economic theory relevant to understanding and exploring deficits in hospital OAT delivery.

9. Section 2.9 contextualizes hospitals in the broader health services delivery market.

10. Section 2.10 summarizes a health services delivery research framework that contributes to the study's conceptual framework (the Health Care Access Framework).

11. Section 2.11 outlines how OAT is delivered outside the hospital.

12. Section 2.12 describes how OAT is delivered within an integrated health services delivery system, the Veterans Health Administration (VHA).

13. Section 2.13 provides an overview of hospital OAT delivery.

14. Section 2.14 synthesizes and integrates the literature reviewed in this chapter to construct the study's conceptual framework.

15. Section 2.15 reiterates chapter conclusions. 


\section{Section 2.1. Defining Drugs, Drug Use Disorders, and Treatment}

For the purpose of this dissertation, drugs are defined as legal or illegal psychoactive substances (e.g., opioids, cocaine) that are "capable of influencing brain systems linked to reward and pleasure" (Babor et al., 2010, p. 9). This dissertation is focused on a psychoactive class of substances called opioids. Opioids are endogenous and exogenous pain-relieving substances that humans have used for thousands of years (Trescot, Datta, Lee, \& Hansen, 2008). Opioid is a broad contemporary term describing compounds with pharmacological effects at opioid receptors primarily in the central nervous system (mu, delta, kappa) (Trescot et al., 2008). An outdated term, frequently used in policy, is the word "narcotic", originating from the Greek word for stupor (Trescot et al., 2008). The term initially described any medication that promoted sleep, then referred more specifically to opioid products, and finally became used in laws and regulations the describe drugs of abuse (Trescot et al., 2008).

All opioids have similar pharmacological effects, such as the capacity to relieve pain, produce euphoria, induce respiratory depression, drowsiness, and impair judgment (Babor et al., 2010). Continued use of opioids leads to tolerance and may result in an OUD. According to the American Psychiatric Association (APA) (APA, 2013), OUD occurs when a person has: "A problematic pattern of opioid use leading to clinically significant impairment or distress," and the person meets at least two of the 11 listed criteria (e.g., craving, unsuccessful efforts to cut down) within a 12-month period. A fulldefinition is in Appendix A. 
The recommended first-line treatment for OUD is OAT (World Health

Organization, 2009, 2017). OATs are long-acting opioids that occupy the mu-opioid receptors in the central nervous system to prevent withdrawal and reduce cravings associated with OUD (Schuckit, 2016). OAT decreases mortality risk and improves treatment outcomes (Sordo et al., 2017). The two FDA approved OATs, available in a variety of formulations, are buprenorphine (with and without naloxone; sublingual tablet, sublingual film, long-acting injectable, and implant) and methadone (oral solution).

\section{Section 2.2. Social Construction of Drugs}

This section introduces and applies social constructionism as it relates to the historical and contemporary discourse of drug use disorders. Social constructionism draws upon the foundational scholarship of Spector and Kitsuse (1977), Foucault (1966), and Turner (1992). Social construction is broadly understood as the "multiplicity of social forces that combine to create and modify [a] phenomenon" (Brown, 1995, p. 37). In the context of health services delivery, medical historian Charles Rosenberg states "[a] disease does not exist until we have agreed that it does" (Rosenberg, 1989, p. 1). Disease creation, moreover, occurs through the generation of specific verbal constructs reflecting the intellectual and institutional history of medicine within the era's social and political context (Rosenberg, 1989). From this approach, the disease is a "social actor" (Rosenberg, 1989) and its application to an individual or group of people can "change 
the narrative and thereby rearrange how we understand the person or behavior" (Reinarman \& Granfield, 2014, p. 2).

In medical sociology, a social construction lens critically evaluates the illness identification process through the influence of social stratification and an assessment of the contributions of health professionals, health service delivery facilities, the government, the media, pharmaceutical companies, other industry profit makers, people with the illness, and their families (Brown, 1995). This approach is useful for studying interactions among the micro (i.e., individual), the meso (i.e., the institutional), and the macro (i.e., the governmental) levels (Brown, 1995). A social construction analysis begins with an exploration of five underlying assumptions about the problem: 1) What are the origins of problem representation?; 2) What elements are missing from the problem?; 3) Are there other ways of thinking about the problem?; 4) In what context does the production, dissemination, and defense of the problem occur?; and 5) What are alternatives to thinking about the problem? (Bacchi, 2012).

For drug use disorders, two common social constructions reflected in historical and contemporary discourse, institutionalized through research, policy, and practice are the moral and the addiction as disease models. The foundational premise of each model, respectively, is that addiction is a deviant behavior or an illness. From a social construction frame, deviant behavior includes the acts, beliefs, and characteristics that violate predominant social norms and attract condemnation, stigma, social isolation, censure, and punishment (Clinard, 1957; Clinard \& Meier, 2011; Goode, 2015a, 2015b). 
Sociologists argue that it is "not a quality of the acts the person commits, but rather a consequence of the application or rules and sanction to an 'offender'"' (Pfohl, 1994, p. 345) and that certain behaviors are categorized as deviant because of the "political and social artifacts of human activity" (Boyd, Carter, \& Macpherson, 2016, p. 8). These scholars reject the premise that punishment deters crime and question how the inequitable exercise of power in society produces crime and deviance (Boyd et al., 2016). A summary of the two socially constructed drug use disorder models, and a third, less common and emergent model, a multi-sourced model of addiction, is presented in the following sub-sections.

2.2.1. The moral model. The central thesis of the moral model is that addiction is a disease of will (Valverde, 1998). Moralists assert that the use of illegal drugs is inherently bad, that drug use and dependency is a consequence of poor decision-making and a lack of personal discipline (Boyd et al., 2016). The moral model is intertwined with the criminal model, which associates substance use disorders (SUDs) with deviance and criminality (Boyd et al., 2016). The moral model came into popular thinking in the $19^{\text {th }}$ century bolstered by the proponents of alcohol prohibition (Reinarman \& Granfield, 2014). Temperance advocates popularized the idea that the deviant behavior behind every excessive drinker was a corrupt moral character and that anyone who drank alcohol was at risk of succumbing to this evilness (Reinarman \& Granfield, 2014). This discourse, in part, led to alcohol prohibition in 1919, and dominated the substance use 
discourse until the early to mid-20 ${ }^{\text {th }}$ century (Reinarman \& Granfield, 2014), informing and shaping contemporary policies and practices related to drug use.

2.2.2. Addiction as disease model. Scholars assert that the development of the addiction as disease model represents a historical shift from one system of social control to another (i.e., church and state to science and medicine) (J. Schneider, 2014). This shift occurred in parallel with the consolidation and accumulation of power by physicians and the growing dominance of the health care industry (Boyd et al., 2016). In contrast with the moral model, the addiction as disease model explains the loss of self-control that occurs with addiction as a pathophysiological process versus a byproduct of the moral failing of the spirit (Reinarman \& Granfield, 2014). The addiction as disease model emerged during the mid- $20^{\text {th }}$ century, a time in which the medicalization of deviant behavior was common (e.g., mental illness) (Reinarman \& Granfield, 2014). The invention of brain imaging technology in the 1990s led to the latest iteration of the addiction as disease model: the brain disease model (Reinarman \& Granfield, 2014). Founders of the brain disease model assert that SUDs are explained by the changes in brain structure and function; addiction is, therefore a chronic progressive, permanent, relapsing brain disorder (Leshner, 1997; Volkow \& Collins, 2017; Volkow, Koob, \& McLellan, 2016).

2.2.3. Multi-sourced model of addiction. An emergent field of scholarship, critical addiction studies, posits that the historical, cultural, and contextual specificity of drug use contribute to the development and visibility of SUDs (Reinarman \& Granfield, 
2014). This scholarship emerged in the latter half of the $20^{\text {th }}$ century to challenge the dominant brain disease model discourse (Reinarman \& Granfield, 2014). Emerging from the critical addiction studies approach was the multi-source model of addiction (Kovac, 2014). This model is grounded in theory across disciplines (i.e., philosophy, sociology, biochemistry, neurobiology, physiology, genetics, political science, economics, and psychology) and asserts that there are five interacting contributory processes at the macro and micro-level leading to addictive behaviors: 1) past actions and current choices; 2) pre-dispositions; 3) social, historical, and cultural environment; 4) neurobiology; and 5) underlying processes (Kovac, 2014). This model does not favor one mechanism as the primary cause of addiction and emphasizes that every case is a unique combination of circumstances (Kovac, 2014). This broader lens of addiction etiology is further supported in forthcoming sections in this chapter that include the macro-level historical, cultural, and contextual perspectives.

Recognizing that different socially constructed models of addiction exist facilitates a nuanced interpretation of contemporary opioid control policies and regulations, and explains, in part, contemporary OUD care. As aptly stated by Dr. Walter Ling: "From the very beginning our policy has been: Addicts are sick, they need help; but they also sin and must suffer a little. So, we built treatment programs and put up barriers making it difficult for patients to get into treatment" (Ling, 2016). Relevant to this dissertation is the influence of the social construction of opioids (e.g., illicit drug or medicine) and opioid users (e.g., morally deficient or patients). The social construction 
of drug use disorders is the meta-influencer of the dissertation's rationale and research design. The next section explores the theoretical connection between social construction and policy design.

\section{Section 2.3. Policy Frameworks}

This section provides complementary and additive theoretical support that social construction and contextual phenomena influence contemporary policy and practice related to hospital OAT delivery. Three policy frameworks are reviewed: The Social Construction Framework, the Policy Feedback Theory, and the Comparative Drug Policy Analytic Theory.

2.3.1. The Social Construction Framework. The underlying assumption of the Social Construction Framework (Ingram \& Schneider, 1990, 1991; A. L. Schneider \& Ingram, 1988, 1993) is that policies define and determine who receives societal benefits or burdens (A. L. Schneider, Ingram, \& deLeon, 2014). Policymakers use socially constructed target populations as justification for policy design and do this through the presentation of powerful images or stereotypes that become embedded within the policy (A. L. Schneider et al., 2014).

This framework has five propositions. First, benefit or burden allocation depends on the target population's political power, which may be positive or negative and highpower or low-power (A. L. Schneider et al., 2014). The power typology for substance users is low-power and negative; thus, under this framework people who use drugs are undeserving of benefits. Second, the framework asserts that policy design has material 
and symbolic effects on the target population (A. L. Schneider et al., 2014). Policy design may impact a group's attitude and political participation through the creation of structures that influence life opportunities (A. L. Schneider et al., 2014). Third, emotional and intuitive reactions (i.e., biases) are at the origins of social construction, and these biases justify the selection of evidence to support policy design (A. L. Schneider et al., 2014). The fourth proposition is that social construction may change frequently through "unanticipated or unintended consequences of previous policy designs" (A. L. Schneider et al., 2014, p. 124). Note, that changing the narrative for a negatively constructed group of people is challenging, and frequently the target population continues to exist as "a negative degenerative social memory" (A. L. Schneider et al., 2014, p. 125). The fifth proposition is that future policy change is dependent on the social construction of the target population (A. L. Schneider et al., 2014).

2.3.2. Policy Feedback Theory. The grounding premise of the Policy Feedback Theory (Pierson, 1993; Skocpol, 1992) is that other policies influence policy design. This theory asserts that policy creation is "deeply influenced" by the existence of other policies that shape political landscapes, foster partisan identities, provide precedent, build governmental capacities affecting policymaker choice, and the dictation of administrative arrangements (Mettler \& SoRelle, 2014). This theory is grounded in historical institutionalism, an approach that views policies as possessing the attributes 
of formal institutions; thus, policies may bestow resources, impose coercive rules, and convey messages and norms (Mettler \& SoRelle, 2014).

The Policy Feedback Theory's four proposed mechanisms that lead to policy adaptation over time include that policies: 1) can shape the meaning of citizenship; 2) can affect the form of governance; 3) can influence the power of groups; and 4) can affect political agendas and policy problem definition (Mettler \& SoRelle, 2014). Moreover, policies can have resource effects (e.g., increased education increases civic engagement) and interpretative effects (e.g., constructed identities may be normative and evaluative or positive or negative) (Mettler \& SoRelle, 2014).

2.3.3. Comparative Drug Policy Analytic Framework. The Comparative Drug Policy Analytic Framework, like the Policy Feedback Theory, proposes that drug policy is influenced through dynamic and interactive relationships with other social phenomena (MacCoun et al., 1993). Scholars of this framework identified three influencers: 1 ) the social context (i.e., the attitudes, norms, economics, demographics of a community); 2) the ongoing drug problem (i.e., community rates of addiction, overdose, criminality, and drug-related accidents); and 3) the existence of other social policies (i.e., health policy, welfare policy, and citizenship rights) (MacCoun et al., 1993).

2.3.4. Summary. These frameworks, as a collective, support the assertion that the social construction of drug users and other contextual phenomena influence the design, implementation, and enforcement of contemporary opioid and OUD-related policies. Moreover, the frameworks provide the rationale that policies confer either 
benefit or burden to persons with OUDs through delivery system design (e.g., methadone clinics), the distinction of criminal versus patient (e.g., federal drug scheduling laws), and access to treatment (e.g., insurance coverage).

\section{Section 2.4. History of OUD Treatment: Policy and Practice}

This section highlights the influence of historical policies and practice on contemporary opioid-related policies and treatment systems. The review begins in the $19^{\text {th }}$ century because prior to this time opioid importation and consumption occurred with public indifference and little government involvement. Over the course of the $19^{\text {th }}$ and $20^{\text {th }}$ centuries, specific opioid products were designated as legitimate or illegitimate through policies attributed to different groups of people. These shifting regulatory designations determined that some opioids were taxable and tradeable (e.g., raw opium), that some opioids were medicine (e.g., morphine), and that some opioids were illegal (e.g., smoking opium, diacetylmorphine).

2.4.1. Opium, morphine, and diacetylmorphine. Prior to the advent of modern chemistry and the pharmacy distribution system, there was opium. Opium was sold in general stores and groceries as a medicinal product (Aurin, 2000). Opium was the first federally regulated opioid. Beginning in the late $19^{\text {th }}$ century, smoking opium was banned from importation from China (1880), was taxed domestically (1890), and eventually designated an illegal substance (1909) with criminal sanctions for possession except for medical purposes (Martin, 1977). Historians attributed the shift of this opioid 
product from taxable good to a socially menacing illicit substance as primarily due to racist anti-Chinese sentiment (J. P. Hoffmann, 1990).

As smoking opium became illegal, the legitimation of opioids through medical practice was flourishing, specifically the use of morphine. In the $19^{\text {th }}$ century opioid medicalization was possible because of the advent of modern chemistry, which allowed for the compounding, production, and marketing of opioid products to druggists and physicians. In 1803, a German pharmacist isolated morphine from the juice of the opium poppy (Musto, 2002), and 30 years later, in 1832, morphine manufacturing and distribution began (Musto, 1973). By mid-century, morphine was a common medical treatment (Musto, 2002) ingested either orally or intravenously (Musto, 1999).

From 1870 to 1880 , the U.S. per capita opioid consumption nearly tripled (Courtwright, 2015). Increased use was attributed to self-medication, the advent of opioid-laced patent medications, disease and trauma from the Civil War, the spread of opium smoking, the promotion of new drugs, and physicians providing and compounding opioids (Courtwright, 2015). The result of increased opioid access and use culminated in the 1870 s morphine crisis primarily afflicting affluent white women (Courtwright, 2015).

Although this opioid crisis was discussed in the public discourse, federal policies were not implemented to delegitimize morphine or to support the treatment of persons with morphine addiction. During this era, the late $19^{\text {th }}$ century and early $20^{\text {th }}$ century, persons with OUD could receive treatment from a scattered collection of private 
physicians, rest homes, private hospitals, or state asylums (White, 2002). The primary treatment provided in those facilities were withdrawal protocols using other psychoactive substances, such as cannabis, cocaine, alcohol, other opioids, and sedatives (White, 2002). The most common approaches were: 1) abrupt opioid withdrawal over 24 to 36 hours; 2 ) rapid opioid withdrawal over four to 10 days; or 3 ) gradual withdrawal over a period of weeks or months (White, 2002). Generally, these modalities were ineffective and return to use was common (White, 2002).

Ironically, the morphine crisis may have contributed, in part, to diacetylmorphine development at the end of the $19^{\text {th }}$ century. Chemists sought to reduce the negative side effects of morphine (e.g., dependency, constipation) and expand the positive effects (e.g., cough suppression) (Musto, 2002). Scientists at Bayer Pharmaceuticals extracted and manufactured diacetylmorphine as a morphine derivative in 1898 (Moynihan, 2002). It was imported to the U.S. as a cough suppressant for pneumonia and tuberculosis (Musto, 2002), and also as a treatment for morphine dependence (White, 2002).

2.4.2. The 1906 Pure Food and Drugs Act. At the turn of the $19^{\text {th }}$ century medicalized opioids were largely unregulated and widely available, and druggists and physicians became concerned (Spillane \& McAllister, 2003). Health professionals feared "becoming slaves to an industry whose desire for profit would overwhelm care for public health and safety" and sought to protect the drug supply by proposing the creation of a regulatory system requiring the recommendation or prescription by a 
physician for the use of opioids and other substances (Spillane \& McAllister, 2003, p. 6). The powerful coalition of physicians and druggists contributed to the political support necessary to pass the 1906 Pure Food and Drug Act (Spillane \& McAllister, 2003).

The 1906 Pure Food and Drug Act established the U.S. domestic prescription drug policy regime. The Act regulated drug promotion, packaging, distribution of specific compounds, and created the first list of restricted "dangerous drugs" including morphine, opium, and diacetylmorphine (Spillane \& McAllister, 2003). In addition, enforcement of the Act occurred under the authority of the Federal Bureau of Chemistry, an organization that would later become an important opioid treatment regulator: the U.S. Food \& Drug Administration (FDA) (Swann, 1998). The authority of the FDA grew with the 1938 Food, Drug, and Cosmetic Act, expanding the agency's authority to oversee cosmetics and medical devices, and mandated labeling for drugs with directions for safe use, and the pre-market approval of all new drugs (Swann, 1998). Under this new regulatory system manufacturers had to prove to the FDA that a drug was safe before it could enter the market (Swann, 1998).

2.4.3. The narcotic clinics. At the same time as the development of the pharmaceutical regulatory system a more robust addiction treatment system began to form without the support of the federal government, the narcotic clinics (White, 2002). The narcotic clinics, created by physicians, were the first community-based facilities dedicated to providing OAT for patients with OUD (White, 2002). Patients with severe OUD would receive OAT to "stabilize" their condition and patients who were more 
"able-bodied" would undergo gradual outpatient withdrawal (White, 2002). The purpose of the clinics was to provide consistent medical management of OUD, to suppress the illegal drug market, and to prevent "drug peddlers" from selling opioids to people with addiction (White, 2002). The clinics ranged from effective and reputable to ineffective and disorganized (White, 2002).

2.4.4. The 1914 Harrison Narcotic Act. In 1912, international delegates convened at the Hague Convention to establish a worldwide supply control standard for the manufacturing and trade of raw opium, morphine, diacetylmorphine, and cocaine (Martin, 1977). U.S. policymakers used the momentum and policy design from the 1912 Hague Convention to enact the 1914 Harrison Narcotic Act. The 1914 Harrison Narcotic Act regulated the importation, manufacturing, and distribution of psychoactive substances as determined by the 1912 Hague Convention (Sacco, 2014). The Act required persons involved in any part of the drug distribution processes to register with the U.S. Department of the Treasury, pay special taxes, and to track all transactions (Sacco, 2014). The Act technically allowed physicians to prescribe opioids and cocaine for treating persons with OUD; however, in contravention of the text, many physicians were arrested, prosecuted, and incarcerated for providing diacetylmorphine or morphine as a treatment for OUD (Sacco, 2014).

A series of three U.S. Supreme Court cases reviewed in 1916 and 1919 challenged the Act's legality: Jin Fuey Moy v. United States (1916); Webb et al. v. United States (1919); and United States v. Doremus (1919) (Hohenstein, 2001). In Jin Fuey Moy 
v. United States, the Supreme Court overruled a lower court ruling that Dr. Moy, a physician who provided opium for a non-registered patient did not violate the provisions of the Act (Hohenstein, 2001). Three years later in Webb et al., v. United States and United States v. Doremus the U.S. Supreme Court affirmed the rulings of the lower courts finding the physicians in violation of the Act because the doctors had provided opioids to "dope fiends" (Hohenstein, 2001). Hereinafter, according to the Supreme Court, only physicians who prescribed opioids to their patients as medical treatment, versus for their addiction, could avoid the provisions of the Act (Hohenstein, 2001). Contemporary OAT policies reflect this foundational ruling-it is illegal, in most clinical scenarios, for health care professionals to prescribe opioids for OUD treatment, unless a specific product has received FDA approval for that indication. In addition to federal action to delegitimize the use of specific opioids, in 1920 the American Medical Association's (AMA) House of Delegates passed a resolution banning the use and importation of diacetylmorphine for medical purposes (Courtwright, 2002).

The culmination of these policies and resolutions resulted in the destruction of the narcotic clinics (AMA: Council on Mental Health, 1966). By the end of 1920, all narcotic clinics were closed because of prosecutorial threat; as a result, in less than a decade the "fledging specialty of addiction medicine was all but obliterated" (White, 2002, p. 136). It is at this point in history that many subsequent opioid-related federal policies shifted from taxable product to the criminal sanctions policy regime (White, 2002). In 1924, Congress enacted the Heroin Act, which created a federal prohibition for 
importing, manufacturing, and possessing diacetylmorphine, including for medical use, and criminalized all persons who used, sold, or administered diacetylmorphine (Musto, 1973).

2.4.5. Early treatment in hospitals. From 1924 to 1935, after diacetylmorphine distribution and use became illegal, and the community-based narcotic clinic system collapsed, OUD treatment resources were limited (White, 2002). Treatment access was dependent on the race and social class of the patient. Affluent middle-aged patients sought discreet withdrawal management in private hospitals. Young people with heroin addiction, in contrast, were more likely to undergo opioid withdrawal in a correctional facility (White, 2002). Physicians practicing in private and community-based hospitals continued to focus on opioid withdrawal management resulting in the development of several withdrawal regimens, such as the "Towns-Lambert Treatment," the "Pettey Method," and the "Nellens and Masse Method" (White, 2002). Eventually, the health care professional community recognized that these protocols were ineffective for curing addiction (White, 2002), and hospitals began denying OUD patients access, resulting in a substantial increase in people with OUD entering the criminal justice system (White, 2002).

Moreover, a eugenics movement successfully lobbied for the inclusion of "addicts" in state mandatory sterilization laws, and inebriate commitment laws were expanded to provide for the involuntary commitment of persons addicted to opioids to state asylums (White, 2002). These practices reflected the socially constructed policy 
targets of the 1924 Heroin Act, which dictated that persons who used specific opioids (i.e., diacetylmorphine) were criminals and immoral. One of the consequences of this shifting discourse was the invention of cruel and invasive interventions for OUD such as "serum therapies," which created blisters on a person's body and withdrew fluid and reinjected it into the blisters (Reddish, 1931) or "bromide sleep treatments" that had a $20 \%$ death rate (Church, 1900).

2.4.6. The 1929 Porter Act and the narcotic farms. By 1928, persons with addiction comprised nearly two-thirds of federally incarcerated persons, because of facility crowding Congress legislated the creation of specialized addiction treatment hospitals (White, 2002). The Porter Act, with support from Dr. Lawrence Kolb of the U.S. Public Health Service, approved the construction of two "narcotic farms" one in Lexington, Kentucky, and the other in Fort Worth, Texas (White, 2002). Together, these facilities had the capacity to treat 2,400 criminally-justice involved persons and voluntary patients (White, 2002). The hospitals divided treatment into three phases: withdrawal, convalescence, and rehabilitation (White, 2002). Interprofessional teams of physicians, nurses, social workers, chaplains, and recreational therapists administered treatment modalities within the hospitals (White, 2002). Once patients completed their opioid withdrawal protocol they spent their time working on the farm (White, 2002). There was limited programmatic success as 90 to $95 \%$ of patients returned to drug use upon leaving the farms (Maddux, 1978).

The testing of many contemporary OUD treatment modalities, such as 
methadone, occurred at the farms as part of the Addiction Research Center (ARC) (Campbell et al., 2008). However, most of the research practices would be considered unethical by $21^{\text {st }}$ century human subjects research protection standards. Until 1955 , for example, patients would receive drugs in return for their participation in experimental studies (Campbell et al., 2008) and these studies were conducted primarily on incarcerated individuals. The narcotic farms, up until the 1960s, were the primary addiction treatment facilities in the U.S. (White, 2002). Operations changed significantly with the passage of the 1966 Narcotic Addict Rehabilitation Act (Campbell, 2010), and in 1974, the hospitals were closed and converted into minimum security prisons (White, 2002). The history of the ARC post-narcotic farm is convoluted but the research program was eventually absorbed as part of the broader intramural research program at the National Institute on Drug Abuse (Campbell et al., 2008).

During the narcotic farm era, some states experimented with state-operated addiction treatment hospitals or created "addict wards" in community hospitals (White, 2002). These experimental delivery systems, however, failed to spread across the U.S. Subsequently, two other outpatient treatment delivery approaches emerged: the therapeutic community and methadone maintenance therapy (White, 2002). The therapeutic community model started in 1958 with the creation of a mutual aid group called Synanon (White, 2002). This model required participants to commit to one to two years of "re-socialization" through authoritarian surrogate families (White, 2002). By 1975, there were more than 500 therapeutic communities in the U.S. (Mitchell, Mitchell, 
\& Ofshe, 1980; Yablonsky, 1967).

German scientists synthesized methadone during World War II (Kleber, 2002) and it was FDA approved for the treatment of pain 1947 (Institute of Medicine Committee on Federal Regulation of Methadone, 1995). Methadone has a longer duration of action than other opioids and a single dose is sufficient to prevent opioid withdrawal for up to 24 hours. Methadone maintenance in community-based treatment centers emerged in the 1960s (Kleber, 2002). Drs. Marie Nyswander and Vincent Dole pioneered this treatment delivery mechanism and conceptualized OUD as a metabolic disease that necessitated the daily oral administration of methadone to stabilize the metabolism (Kleber, 2002).

During the 1960s, the social construction of drug use disorders shifted towards the addiction as disease model. In 1961, a joint committee of the American Bar Association and the AMA questioned the long-standing repressive drug policies of the prior forty-years and encouraged OAT research (Musto, 1987). In 1962, the U.S. Supreme Court in Robinson v. California declared that narcotic addiction was a disease and that persons with addiction should be "proper subjects for medical treatment" (White, 2002). The 1966 Narcotic Addict Rehabilitation Act reflected this change in the social construction of drug users. The Act authorized, treatment of "narcotics addicts" in lieu of incarceration for federal drug offenses and promoted the development of community-based outpatient services (Besteman, 1992).

2.4.7. International drug conventions. International treaties are the foundation 
of contemporary U.S. opioid regulations. In 1925, at the International Opium Convention delegates created the first psychoactive regulatory mechanism for opioids and other drugs (League of Nations, 1925). These efforts were expanded upon with the 1931 Manufacturing Convention (League of Nations, 1931), which was strongly influenced by a turbulent social environment. First, the convention occurred during a worldwide economic recession, the European banks were failing, countries were experiencing currency destabilization, and foreign trade had "imploded" (McAllister, 2004). Thus, delegates did not want to impose limits on the export of profitable medicalized opioids (e.g., codeine in Germany) because countries needed the revenue (McAllister, 2004). Second, international delegates received conflicting pressure from scientists, who were concerned about policies limiting research capabilities; and temperance advocates, who wanted the strictest limits on drugs (McAllister, 2004). Third, the moral model dominated the social construction of drug users at this time; thus, delegates believed if the illicit drug supply was eliminated drug misuse would end (McAllister, 2004). The delegates created a two-tiered regulatory structure for psychoactive substances to meet the needs of all of their stakeholders and to adhere to the socially constructed narrative of drug users at the time: Group I (e.g., morphine, heroin, cocaine) and Group II (e.g., codeine) (League of Nations, 1931). The policy required that countries track Group I drugs during retail transaction and distribution (McAllister, 2004).

Thirty years later, at the 1961 United Nations (UN) Single Convention on Narcotic 
Drugs, delegates from 97 nations extended prior regulatory efforts from the Manufacturing Convention and designed and ratified the contemporary prescription policy regime (UN, 2013). The treaty's primary intention was to create worldwide opioid prohibition, except for medical and research purposes (UN, 2013). Convention representatives crafted the regulations with two assumptions: 1 ) limiting the worldwide opioid supply would eradicate illicit use; and 2) opioid control and prohibition would be imposed until evidence demonstrated that a drug did not have addictive potential (Spillane \& McAllister, 2003). The treaty created four drug schedules with different levels of regulation; provided definitions for legal and illegal trade; provided definitions for medical and research purposes; and created penalties for recreational consumption (Spillane \& McAllister, 2003).

2.4.8. Summary. Over the course of the $19^{\text {th }}$ and $20^{\text {th }}$ centuries drug policies institutionalized opioids as a taxable product (e.g., the 1914 Harrison Narcotic Act), illegal substance (e.g., the 1924 Heroin Act), or pharmaceutical treatment (e.g., the UN Conventions) and subsequently categorized specific opioid users as patients or criminals. These policies shape the contemporary treatment policy regimes, dictating how, when, and where persons with OUD can access treatment.

\section{Section 2.5. Contemporary Drug Policy: Regimes, Law, and Regulations}

This section provides an overview of the contemporary drug policy regimes, and

$$
\text { U.S. law and regulations informing access and delivery of OAT. }
$$


2.5.1. Drug policy regimes. Policy regimes are collections of policies intended to achieve a broad goal (Birkland, 2015). Drug policies are trans-disciplinary (Babor et al., 2010; Boyd et al., 2016; MacCoun et al., 1993) and enact a broad range of administrative actions related to substance use. Babor et al. (2010) identified five drug policy regimes: 1) criminal sanctions; 2) health and social services; 3) prescription; 4) prevention; and 5) supply control. Policies may exist in multiple regimes or sub-regimes. The purpose of drug policies is to achieve three goals: 1) prevalence reduction (i.e., decreasing the number of users); 2) quantity reduction (i.e., reducing the amount consumed); and 3) micro-harm reduction (i.e., reducing the average harm per dose to users and non-users) (MacCoun \& Martin, 2008). Each micro-goal contributes to the broader overarching goal of macro-harm reduction, which is to decrease the total harm of drug use to society (MacCoun \& Martin, 2008).

2.5.1a. Criminal and punitive sanctions regime. The criminal and punitive sanctions policy regime includes policies intended to deter drug use, to prevent the normalization and spread of drug use, and to prevent the negative effects of criminalizing less harmful forms of drug use (Babor et al., 2010). This regime includes policy strategies such as deterrence (e.g., threatening to arrest), incapacitation (e.g., incarceration), rehabilitation (e.g., coercive treatment), and penalty modification (e.g. decriminalization, depenalization) (Babor et al., 2010).

2.5.1b. Health and social services regime. The health and social services policy regime includes policies that promote treatment and recovery modalities for persons 
with SUDs from a non-punitive approach (Babor et al., 2010). Treatment policies, a policy sub-regime, affect treatment access through planning, financing, and monitoring of addiction services, as well as the development of the professional workforce (Klingemann, Holder, \& Gutzwiller, 1993; Klingemann \& Klingemann, 1999). Regulatory and allocative treatment policies; moreover, may determine the structural resources available to treat SUDs, such as the number of facilities, the types of programs (e.g., opioid treatment programs [OTP]), the delivery setting (e.g., hospitals), and the health care professionals who can deliver services (e.g., prescribers). Additionally, treatment policies may affect where services are located, and the organization and integration of services (Klingemann et al., 1993; Klingemann \& Klingemann, 1999).

2.5.1c. The prescription regime. For opioids, the prescription policy regime is "the major control structure through which these substances are made legally available for consumption in the modern world" (Babor et al., 2010, p. 179). The two goals of this regulatory system are to permit opioid use for approved medical purposes and to prevent non-approved use (Babor et al., 2010). The prescription regime, although less than a century old, is the normative approach for pharmaceutical regulation globally (Babor et al., 2010). The prescription regime is influenced by consumer groups, international conventions, government regulations, and professional associations (Babor et al., 2010).

2.5.1d. The prevention regime. The prevention policy regime includes policies intended to reduce SUD incidence (Kolodny et al., 2015) by preventing drug use by 
changing attitudes and improving health literacy (Babor et al., 2010). Prevention efforts to decrease OUD and opioid exposure may include policies to support educational programming for prescribers, patients, families, and the public. For example, some states (e.g., lowa, Kentucky, Massachusetts, Ohio, Tennessee, and Utah) mandate provider opioid education (Executive Office of the President of the United States, 2014). Another prevention policy approach is to decrease the number of opioids in circulation with the purpose to reduce long-term exposure and new exposure to opioids (Kolodny et al., 2015).

2.5.1e. The supply-control regime. The primary goal of the supply-control policy regime is to keep illegal drug prices high and to reduce illegal drug availability (Babor et al., 2010). Commonly used supply control approaches include polices for alternative crop development, crop eradication, precursor chemical control, interdiction, high-level enforcement through criminal investigations, street-level enforcement, and imprisonment (Babor et al., 2010).

2.5.2. Contemporary laws and regulations. The $20^{\text {th }}$ and $21^{\text {st }}$ century opioidrelated policies germane to this dissertation cross all drug policy regimes and build upon the foundations of previously reviewed historic policies. The result is a complex contemporary regulatory environment comprised of discordant rules for specific opioids that is shaped by social construction. This section includes a summary of contemporary OAT-related policies and regulations. 
2.5.2a. The 1970 Comprehensive Drug Abuse Prevention and Control Act. This

Act is the cornerstone of contemporary opioid regulations with elements from all five policy regimes (Babor et al., 2010). The enactment of this legislation reflected assorted interrelated social phenomena including the actions taken by President Richard Nixon. As a candidate, Nixon ran on a platform of domestic crime control and, in his view, the primary cause of increased crime was SUDs (Courtwright, 2004). Within the first six months of his presidency, in July 1969, President Nixon submitted to Congress the legislative architecture of what would eventually become the 1970s Comprehensive Drug Abuse Prevention and Control Act (Courtwright, 2004). The proposed legislation, addressed multiple aspects of SUDs through supply control, education, research, treatment, and training (Courtwright, 2004).

At the time, methadone was the only medication available for persons with OUD. Patients received methadone at experimental treatment centers. To circumvent the federal prohibition of treating persons with OUD with opioids, methadone maintenance treatment centers operated as research facilities using Investigational New Drug (INDs) waivers from the FDA (Jaffe \& O'Keeffe, 2003). Between 1967 and 1970 several thousand patients received methadone in these research programs (Institute of Medicine Committee for the Substance Abuse Coverage Study, 1990; Jaffe, 1975; Jonnes, 1996; Kreek \& Vocci, 2002). In June of 1970, the FDA proposed a new rule for methadone IND applications with strict requirements on entry into treatment, dosage, and duration of treatment (Jaffe \& O'Keeffe, 2003). With this rule in place the FDA 
allowed methadone to be more restrictively used but still "thinly disguised as research" (Jaffe \& O'Keeffe, 2003). Other interventions for OUD in the 1960s and 1970s included civil commitment, narcotic antagonists, non-opiate withdrawal medications (e.g., clonidine), and drug testing as a form of treatment monitoring (White, 2002).

On October 27, 1970, President Nixon signed the Comprehensive Drug Abuse Prevention and Control Act of 1970 into law repealing and replacing the long-standing 1914 Harrison Narcotic Act. This omnibus bill included three titles. Title I authorized the Department of Health, Education, and Welfare to engage in drug use prevention and treatment through community mental health centers and public service hospitals, and authorized increased research funding through the National Institute of Mental Health. Title II, named the 1970 Controlled Substance Act, provided the regulatory framework for opioids and other psychoactive drugs. Title III addressed importation and exportation of controlled substances.

The 1970 Controlled Substances Act, informed by the scheduling structure created in the earlier international conventions, provided a regulatory mechanism for categorizing "controlled substances" into five schedules based on acceptable medical use and the potential for abuse, which is in place today:

- Schedule I substances have no currently accepted medical use in the U.S. and have a high potential for abuse (e.g., diacetylmorphine);

- Schedule II substances are considered dangerous, have a high potential for abuse which may lead to severe psychological or physical dependence (e.g., methadone); 
- Schedule III substances have less potential for abuse than Schedule I or II, but more than Schedule IV, with a moderate to low potential for physical and psychological dependence (e.g., buprenorphine);

- Schedule IV substances have a low potential for abuse and risk of dependence (e.g., lorazepam); and

- Schedule V substances have a lower abuse potential as compared to Schedule IV and consist of preparations containing limited quantities of certain opioids (e.g., Phenergan ${ }^{\circledR}$ with codeine) (DEA, n.d.).

The Act does not describe how drug abuse potential is determined, but does provide a legislated review mechanism for scheduling additional drugs and rescheduling or decontrolling already regulated substances (Courtwright, 2004). At the time, the 1970 Controlled Substances Act was seen as "an overdue and noncontroversial piece of legislation" designed to tighten and clarify a statutory and administrative "mess" because the regulatory system prior to that time was unstable and rapidly changing (Courtwright, 2004, p. 10). The major contribution of the Act was the creation of a common regulatory process that shifted drug control from Congressional action to administrative control (Spillane, 2004).

2.5.2b. The 1972 Drug Abuse Office and Treatment Act. After the enactment of the 1970 Controlled Substances Act, the treatment and prescription policy regime expanded rapidly along with access to OUD treatment. In June of 1971, the Nixon administration decided to mainstream methadone, which led to a 1972 revision of the 1970 FDA regulations (Jaffe \& O'Keeffe, 2003). These regulations created a closed treatment system restricting methadone administration to federally approved facilities and hospital pharmacies (Institute of Medicine Committee on Federal Regulation of 
Methadone, 1995; Jaffe, 1975, 1997; Kreek \& Vocci, 2002). The policy was supposed to tightly control methadone distribution until the treatment became less controversial and more well-studied (Jaffe, 1975, 1997). The regulations were in full effect by 1973, and today, are largely in the same form. Methadone is considered to be "the most highly regulated of all medical treatments" (Strain \& Stoller, 1999).

The 1972 Drug Abuse Office and Treatment Act legislatively empowered the Special Action Office for Drug Abuse Prevention and required this new federal office to support the development of opioid antagonist therapy for OUD treatment (Julius, 1976). Moreover, this Act enacted a federal-state-local partnership requiring shared responsibility in the design, implementation, operation, and evaluation of communitybased opioid addiction treatment programs (White, 2002). In the same year, the Social Security Act Amendment addressed addiction-related conditions and stated that both alcoholism and drug addiction would not qualify as a disability unless the person was receiving "appropriate available treatment in an approved facility" (Ball, 1973). Shortly following that amendment, in July 1973, President Nixon established the Drug Enforcement Administration (DEA) through Executive Order 11727, merging together the Office for Drug Abuse Law Enforcement and the Office of National Narcotics Intelligence in the Department of Justice (Nixon, 1973).

2.5.2c. The 1974 Narcotic Addict Treatment Act. This Act amended the 1970 Controlled Substances Act to legislate DEA authority over the storage and security of addiction treatment drugs (Jaffe \& O'Keeffe, 2003). It also required practitioners and 
treatment sites to annually register with the DEA (Jaffe \& O'Keeffe, 2003) .The Secretary of Health, Education, and Welfare (now the Department of Health and Human Services) retained the responsibility for setting standards for proper professional practice in the medical treatment of addiction (Jaffe \& O'Keeffe, 2003), and today, that responsibility is with SAMHSA.

2.5.2d. The 2000 Drug Addiction Treatment Act. This law is the most important OUD treatment policy of the $21^{\text {st }}$ century because it expanded OAT access. Prior to enactment, federal legislation prohibited the prescribing of opioids for OUD treatment, a holdover from the Supreme Court rulings related to the 1914 Harrison Narcotic Act (McCarty, Priest, \& Korthuis, 2018). The 2000 Drug Addiction Treatment Act permits physicians, after registering with the DEA and completing eight of hours of SAMHSAverified training, to prescribe Schedule III, IV, or V controlled substances that are FDA approved for OUD treatment. Currently, buprenorphine (Schedule III) is the only opioid that meets these requirements. Although buprenorphine was approved by the FDA in 2002, it was discovered more than 40 years ago in the United Kingdom (Courtwright, 2004) and its treatment efficacy was hypothesized in the late 1970s (Jasinski, Pevnick, \& Griffith, 1978). The delay in the use of buprenorphine to treat OUD was due primarily to policymakers obtaining the necessary amendments to the 1970 Controlled Substances Act and the 1974 Narcotic Addict Treatment Act (Jaffe \& O'Keeffe, 2003).

\subsection{2e. The 2016 Comprehensive Addiction and Recovery Act and the $21^{\text {st }}$}

Century Cures Act. The 2016 Comprehensive Addiction and Recovery Act authorized the 
expansion of SUD treatment services and overdose reversal medications, including prevention, OAT, and other non-pharmacological recovery supports. This Act also authorized nurse practitioners and physician assistants to become buprenorphine prescribers through the federal waiver program. Importantly, the $21^{\text {st }}$ Century Cures Act, which was passed a few months later, provided $\$ 1$ billion in funding for the treatment expansion efforts outlined in the 2016 Comprehensive Addiction and Recovery Act (ASAM, 2016), including the State Targeted Response to the Opioid Crisis Grants program (SAMHSA, 2017b).

\subsection{2f. The 2018 SUPPORT for Patients and Communities Act. This law} authorized a variety of interventions (e.g., programs and demonstration projects) to address the opioid overdose epidemic through treatment, prevention, recovery, and enforcement (Musumeci \& Tolbert, 2018). Treatment and recovery highlights germane to this dissertation include (Musumeci \& Tolbert, 2018): A limited repeal of the Institutions for Mental Diseases exclusion; the authorization of grants to academic health centers to develop curricula to support provider buprenorphine waiver attainment; and codifying the ability of qualified physicians to prescribe OAT for up to 275 patients.

2.5.6. Summary. The contemporary federal OAT policy regime, created over the last 45 years, dictates where and when persons with OUD can access OAT, and further, how health professionals and health facilities may prescribe or administer OAT. The OAT policy landscape is likely one of many contributory elements informing how patients 
with OUD are treated during hospitalization. How health services delivery organizations are shaped by internal and external elements (e.g., policies) are explored in the next section.

\section{Section 2.6. Organizational Behavior Scholarship}

Organizations are "social structures created by individuals to support the collaborative pursuit of specified goals" (Scott, 2003, p. 11). The core operational requirements of an organization, include: 1) defining their objectives; 2 ) inducing participants to contribute; 3) controlling and coordinating participant contributions; 4) participant selection, training, and replacing; and 5) working with neighbors (Scott, 2003). Organizations must also devote resources to maintain the organization (Scott, 2003).

2.6.1. Leavitt's Diamond. Four central, inter-related organizational elements are included in Leavitt's Diamond (Leavitt, 1962). This model, recently modified by Scott (2003), asserts that organizations are influenced by the environment and comprised of social structures, goals, technology, and participants (Leavitt, 1962; Scott, 2003).

2.6.1a. Social structures. Social structures are the patterned, regularized interactions or relationships among participants in an organization (Scott, 2003). Social structures may be formal (e.g., specified roles) or informal (e.g., indistinguishable roles) (Scott, 2003) and embody a "duality" (Giddens, 1979), meaning they exist as a medium and an outcome. There are three categories of social structures: normative (Davis, 1949; 
Scott, 2003); cultural-cognitive (Berger \& Luckmann, 1966; Scott, 2003; Weick, 1995); and behavioral (Davis, 1949; Homans, 1950; Scott, 2003).

Normative social structures include organizational values, norms, and role expectations (Davis, 1949; Scott, 2003). Normative structures impose constraints on behavioral structures, shaping, and channeling behavior through mutually held expectations and obligations (Davis, 1949; Scott, 2003). Organizational values are the criteria that inform the selection of goals and behavior; norms are the generalized rules governing behaviors; and roles are the behavior expectations for occupants of specific social positions (Davis, 1949; Scott, 2003).

Cultural-cognitive structures are the beliefs and understandings that participants in an organization share about the nature of their situation, providing a common interpretative framework (Scott, 2003)-an organizational culture. Organizational culture formation occurs through the interaction of observable artifacts, espoused values, and basic assumptions (Kinicki \& Kreitner, 2009; Ostroff, Kinicki, \& Tamkins, 2003) and is passed on to new employees through socialization (Kinicki \& Kreitner, 2009). Organizational culture is structuralized through the creation of reward systems, and enacted through socialization, mentoring, group dynamics, decision-making, communication, influence and empowerment, and leadership (Kinicki \& Kreitner, 2009; Ostroff et al., 2003).

Behavioral social structures are the actual behaviors that occur within an organization (Scott, 2003), comprised of individual or group activities, interactions, and 
sentiments (Homans, 1950). The repetition of actual behaviors into patterns or networks, within an organization, provides rich insight into the understanding of the organizational power structures and the sociometric structure (i.e., who is attracted to whom, and who is rejected by whom) of the group (Scott, 2003).

2.6.1b. Participants. Participants are individuals who, in exchange for inducements, make contributions to an organization (Scott, 2003). Participants are usually involved in multiple organizations, and these outside involvements may constrain or influence the behavior of participants (Scott, 2003). Participants are social actors; thus, it is their energy, ideas, conformity or non-conformity that constitute the shape and structure of organizations and resulting functions (Scott, 2003). The demographic characteristics of participants (e.g., age, gender, ethnicity) may have important consequences for organizational structure and function (Scott, 2003). From an organizational perspective, organizations must be able to relate to a range of participant interests and demands. This broader collection of individuals is often called stakeholders. Stakeholders are affected by the organization and have "legitimate claims on an organization" (Scott, 2003, p. 21).

2.6.1c. Goals. Organizational goals are defined as the desired ends that participants attempt to achieve through task performance that involve culturalcognitive and normative elements (Scott, 2003). From a resource dependency perspective, goals and actions are intended to minimize environmental uncertainties (Hillman, Withers, \& Collins, 2009) and dependencies (Pfeffer \& Salancik, 1978) through 
mergers, vertical integration, creation of joint ventures and other interorganizational relationships, board of director actions, political action, and executive succession (Pfeffer \& Salancik, 1978). Another approach to understanding organizational goals is through the use of Chandler's (1962) definition of strategy: "Strategy is the determination of the basic long-term goals and objectives of an enterprise, and the adoption of courses of action and the allocation of resources necessary for carrying out these goals" (p. 13). This approach includes integrated decisions, actions, or plans that will set and achieve organizational goals; the setting of goals and the achievement of goals are the results of strategic decisions (Chaffee, 1985).

2.6.1c. Technology. Technology may be a place where a specific type of work is conducted, a location in which energy transforms materials (Scott, 2003). Every organization does "work" and has a technology for completing the work (Scott, 2003). In the context of health care delivery, health systems "process people" and the product is healthier or healed individuals (Scott, 2003). Technology may be embedded in physical machines, but importantly, it is also the technical knowledge and skills of participants (Scott \& Davis, 2015). Finally, similar to the "duality" of social structures (Giddens, 1979), technology influences and shapes organizational design, by attempting to match the complexity of an organization's structure with the complexity of its environment and technology (Galbraith, 1982).

2.6.1d. The environment. The physical, technological, cultural, and social milieu are the environment in which organizations exist and adapt (Scott, 2003). No 
organization is completely self-sufficient; thus, it depends on relationships established with the larger external system (Scott, 2003). The environment influences the comprising elements of an organization, the social structures, the participants, the goals, and the technology (Scott, 2003).

Few organizations assume responsibility for training and socialization of their participants; thus, employees join organizations with "heavy cultural and social baggage obtained from interactions in other social contexts" (Scott, 2003, p. 23). Further, few organizations create their own technologies; most are imported from other organizations, including employee rules and professional norms (Scott, 2003). Goals may be influenced by the external environment through societal support and informed by societal values (Scott, 2003). Social structures are also borrowed from the environment, as organizations frequently follow models that already exist (Scott, 2003). It is, therefore, an imperative that studies of organizational behavior include considerations of the larger environment.

Another approach to understanding the influence of the external environment on organizational behavior is institutional theory. This theory asserts that there are three mechanisms in the environment that shape organizations towards homogeneity through a process called isomorphism (DiMaggio \& Powell, 1983). There are three isomorphic mechanisms: coercive, mimetic, and normative (DiMaggio \& Powell, 1983). Coercive isomorphism is caused by external political influence, legitimacy, formal and informal cultural pressures, or governmental mandate (DiMaggio \& Powell, 1983). 
Mimetic isomorphism occurs when an organization mimics another in the environment due to environmental uncertainty (DiMaggio \& Powell, 1983). Normative isomorphism is associated with professionalization, which is the collective struggle by occupational members to define the conditions and methods of their work, control the number of persons entering the profession, and establish a cognitive base and legitimate occupational autonomy (DiMaggio \& Powell, 1983).

\section{Section 2.7. Systems Science Scholarship}

Organizations are coordinated systems producing controlled activities embedded within complex networks participating in technical and boundary-spanning exchanges (Meyer \& Rowan, 1977). There are a variety of system typologies and sub-typologies: rational; natural; open; closed; or a combination (Scott, 2003). For this dissertation, health service delivery organizations are conceptualized as sociotechnical and complex adaptive systems. Sociotechnical systems function as closed (i.e., technical) and open systems (i.e., institutional) (DiMaggio \& Powell, 1991; Scott \& Meyer, 1991; Thompson, 1967). A closed, technical system produces a product or service within a contained environment that is exchanged in a market that rewards the organization for effective and efficient control of the production systems (Scott \& Meyer, 1991). An open system is comprised of inputs, the inflow of external energy and information, throughputs, the energies inside the system, outputs, the exported product, cycles of events, the repeated activities to exchange and transform energy, and negative feedback, the internal information used to correct and adjust activities (Katz \& Kahn, 1978). The 
definition of an open system is "Congeries of interdependent flows and activities linking shifting coalitions of participants embedded in a wider material-resource and institutional environments" (Scott, 2003, p. 29). Complex adaptive systems are "A collection of individual agents with freedom to act in ways that are not always totally predictable, and whose actions are interconnected so that one agent's actions changes the context for other agents" (Plsek \& Greenhalgh, 2001, p. 625).

There is unresolvable tension and paradox within a complex adaptive system reflected in the amalgamation of contradictory properties, which are found in health service delivery settings, including:

- The system has unclear and fuzzy boundaries;

- The system has non-linear behaviors;

- The system has unpredictable outcomes;

- The system has inherent patterning;

- The system has embedded co-evolving systems;

- The agents within the system are adaptive; or

- The agents within the system act based on internalized rules (Plsek \& Greenhalgh, 2001).

Complex systems are comprised of functional units that interact non-linearly with other units within a system (Lipsitz, 2012). Hospitals exist as functional units within the nested context of a larger complex health services delivery systems, including: the patient, the care team, the department, the division, the hospital, the health system, 
the relationships among multiple hospitals and health systems, a system of hospitals, hospital networks, all hospitals, the industry, the region, the nation, and the global community (Ferlie \& Shortell, 2001; Perrow, 1986). The nesting phenomena causes continuous evolution and interaction within each level; thus, theoretical multilevel frameworks examine health behavior interventions (Sallis et al., 2015), health care improvement leverage points (Ferlie \& Shortell, 2001), the health care delivery enterprise, health service utilization (Andersen, Davidson, \& Baumeister, 2013; Levesque et al., 2013) and health care value-networks (Basole \& Rouse, 2008).

Scholars describe the production of health services as existing within a nested context-patient, care team (e.g., frontline health professionals), organization (e.g., facility infrastructure and resources), and the environment (e.g., regulatory, market, and policy frameworks (Fanjiang, Grossman, Compton, \& Reid, 2005; Ferlie \& Shortell, 2001); thus environmental attributes (e.g., regulatory pressures and statutory requirements) may influence hospital structures and hospital attributes (Covaleski, Dirsmith, \& Michelman, 1993; Goodrick \& Salancik, 1996; Ruef \& Scott, 1998).

\section{Section 2.8. Economics Scholarship}

Economic theories and analyses attempt to predict and explain human behavior through the modeling of agents (e.g., individuals, organization) and resources (e.g., labor, monetary) (Feldstein, 2005; Friedman, 2002). A neoclassical economics approach assumes that individual agents are rational, that transactions are governed by market equilibria, that organizations are committed to efficiency, that high profits are evidence 
of efficiency, and that efficiency benefits society (Perrow, 1986). Rationality is articulated as either procedural (i.e., bounded) or substantive (i.e., objective) (Simon, 1985). In bounded rationality, three elements are necessary to understand decisionmaking: 1) the individual's goal; 2) the information and conceptualization of the situation; and 3) the individual's ability to draw inferences from information in possession (Simon, 1985). In objective rationality, only two elements are necessary for understanding choice: the individual's goals and the objective situational characteristics (Simon, 1985). Thus, bounded rationality accounts for the limits of human cognition and recognizes the resources necessary for processing information and making a decision (Simon, 1985).

A transaction, from an economics perspective, is an exchange that occurs between two parties in the market that may incur costs (Friedman, 2002). A market is an allocative mechanism and an institutionally specific cultural system that generates and measures value (Friedland \& Alford, 1991). New market equilibrium situations are predicted and explained using supply and demand principles (Feldstein, 2005). Supply is the quantity of a good sold at a given price and demand is the quantity of a good purchased at a given price (Friedman, 2002). Budget constraints and income level also impact the supply and demand curve (Friedman, 2002). When a price changes along the supply and demand curves, the quantitative impact is called elasticity (Feldstein, 2005). Market equilibrium occurs when consumer quantity demand is equal to supplier quantity, which is a state of economic efficient allocation, meaning organizational 
resources are being used to maximize value to its members (Friedman, 2002). Efficiency is the use of a firm's resources to maximize value to its members through the goods and services produced (Friedman, 2002). In the market, resources are scarce; thus, specific resource allocation involves an opportunity cost, meaning that resources spent on one item are not spent on another (Friedman, 2002).

Resource allocation is either efficient or inefficient (Friedman, 2002). An efficient allocation of resources is one where there is no waste and everyone gains. In reality, there is no perfectly efficient allocation; therefore, the concept of relative efficiency exists. Relative efficiency compares the efficiency of one allocation to another (Friedman, 2002). There is always room to improve efficiency, and efficient allocation does not always mean an optimal or equitable allocation of resources (Friedman, 2002). Efficiency and allocation are the cornerstones of government economic objectives, which are to improve market efficiency and achieve appropriate allocation of resources (Feldstein, 2005).

2.8.1. Agency theory. Agency theory is based on the premise that relationships are represented by contracts between principals and agents (Perrow, 1986). The principal (e.g., the hospital) delegates work to the agent (e.g., the physician) who performs the work (Eisenhardt, 1989). Agency theory has several assumptions. First, people are self-interested, bounded rationally, and risk averse (Eisenhardt, 1989). Second, the principal-agent relationship is fraught with goal conflict and information asymmetry (Eisenhardt, 1989). Third, efficiency is the criterion for effectiveness 
(Eisenhardt, 1989). Agency theory explores conflicts in contract design (Alchian \& Demsetz, 1972; Jensen \& Meckling, 1976; Zeckhauser \& Pratt, 1985), particularly the influence of third-party enforcers (Scott, 1995). Third-party contract enforcers have their own utility functions (North, 1990) and monitoring costs (Zeckhauser \& Pratt, 1985).

2.8.2. The rational actor model. The rational actor model uses the utility maximizing model as a theoretical base, is frequently applied in political analysis, and describes unitary decision-makers (Allison \& Zelikow, 1999). The utility maximizing model is a common framework for understanding resource allocation choices. The utility maximizing model makes four assumptions about the consumer: 1) she has consistent preferences and preference-ordering; 2) she is non-satiable; 3 ) she prefers a specific proportion of goods; and 4) she makes choices based on information and her selfinterest (Friedman, 2002).

2.8.3. Transaction cost theory. Transaction cost theory explains and explores economic transaction design associated with organizations (Williamson, 1994). The transaction cost theory assumptions include two elements: 1) humans will behave opportunistically with guile under a frame of bounded rationality; and 2) there are exchange characteristics that may determine design, such as asset specificity, uncertainty, and frequency of transactions (Williamson, 1979, 1985; Williamson \& Ouchi, 1981). 


\section{Section 2.9. The U.S. Health Services Delivery Market}

This section provides an overview of the U.S. health services production market and explores why care delivery variation persists despite advancements in health care technology.

2.9.1. Health services commodification. The construction of the U.S. health services delivery system reflects the needs of a political and an economic environment that has institutionalized a system of "capitalist relations of production" (Christiansen, 2017, p. 83). From this lens, capitalists seek to "Augment (and realize) surplus value, as well as to increase that portion of surplus value that becomes their profit... while simultaneously offering only those 'benefits' necessary for retaining their workforce" (Christiansen, 2017, p. 83). Under this approach, health service delivery is commodified and private enterprise manages a large portion of health care expenditures and profits (Christiansen, 2017). The primary goals of the health service delivery system are the provision of services and the maximization of profit for shareholders (Christiansen, 2017). The U.S. health care delivery system is "a loose federation of independent enterprises, all trying to optimize the market from their perspective and for their benefit" (Basole \& Rouse, 2008). In 2015, the delivery of health care services accounted for $17.5 \%$ of the U.S. Gross Domestic Product (National Center for Health Statistics, 2017). Out of the total national health expenditure of $\$ 3.0$ trillion, hospital services accounted for $32 \%$ of expenditures (National Center for Health Statistics, 2017). Therefore, in addition to the contributions of organizational and system-level attributes 
as previously described, the U.S. economic production system of health services delivery influences hospital OAT delivery.

The quantification of the economic value of the health services produced in the hospital setting is based on the hospital's self-designated procedure and supply list-the "chargemaster" (Reinhardt, 2006). There are two steps in hospital billing and reimbursement. First, the hospital bills third-party payers or patients directly using the defined prices on the chargemaster; and second, the hospital is reimbursed through a unique negotiated payment contract with a third-party payer or the uninsured patient (Reinhardt, 2006). Third-party payer contracts with hospitals may be based on steeply discounted charges, negotiated per diems, or flat charges per episode dependent on negotiations (Reinhardt, 2006). Medicare reimburses hospital-based services using a flat fee per case payment structure (Reinhardt, 2006) and Medicaid reimburses hospitals using a legislated formula that includes a base payment and a variety of statedetermined supplemental payments (Cunningham, Rudowitz, Young, Garfield, \& Foutz, 2016). One of the most common contractual arrangements in U.S. hospitals, based on the Medicare payment model is diagnostic related grouping (DRG). DRG billing is a contractually agreed upon amount, set between the hospital and the third-party payer, for a specific condition or treatment bundle (Quinn, 2008).

2.9.2. Variation in service delivery. Health services delivery variation, herein referred to as care variation, has been a topic of interest since the first scientific study on tonsillectomy variation in United Kingdom school children (Glover, 1938). From a 
variation perspective, health care services are categorized as: effective (Category I), preference-sensitive (Category II), and supply-sensitive care (Category III) (Chandra \& Skinner, 2012; Skinner, 2011; Wennberg, Fisher, \& Skinner, 2002). Category I care includes highly effective treatments that are inexpensive and productive across the population (e.g., antibiotics for bacterial infections) or are highly productive and expensive for a well-defined group of patients (Chandra \& Skinner, 2012; Skinner, 2011; Wennberg et al., 2002). In general, Category I treatments will eventually diffuse across the system to near-universal use, although this process can be slow (Berwick, 2003). Category I treatments may have substantial impact on health outcomes, but are not likely to play a large role in explaining variation in expenditures (Skinner, 2011) Category II care includes preference-sensitive care-treatments that exhibit considerable benefit heterogeneity across different populations (Chandra \& Skinner, 2012; Skinner, 2011; Wennberg et al., 2002). An example of this type of care is the use of stents, which only benefits a very specific group of people (Hartwell et al., 2005). Category II treatment variation is observed in national health systems with salaried physicians, which suggests that variation is not solely due to the presence of fee-forservice or income-maximization by physicians (Skinner, 2011).

Category III care is supply-sensitive treatments, where there is limited evidence of benefit (e.g., knee arthroscopy) or the benefit is unknown (Chandra \& Skinner, 2012; Skinner, 2011; Wennberg et al., 2002). Skinner (2011) proposes that Category III treatment utilization plays a significant role in explaining expenditure differences across 
regions. Hospital utilization is considered a Category III treatment because the incremental health value of greater hospital capacity is either small, zero or unknown (Fisher, Wennberg, Stukel, \& Sharp, 1994). Skinner (2011) proposes that patterns of geographic variation can be explained in part by the theory of reasoned action (Ajzen \& Fishbein, 1980). In this model, individual behavior is disaggregated into goals and beliefs about how to attain goals (Skinner, 2011). Patients and physicians are assumed to share the same goals: better functioning and longer lifespan for the patient. However, local health care systems may perceive different approaches for attaining those goals (Skinner, 2011). One of the proposed solutions for care variation is to standardize care in clinical scenarios with high levels of certainty, clinical agreement, and definitive science (Institute of Medicine Committee on Quality of Health Care in America, 2001). For the purposes of this dissertation, OAT is a category I treatment.

\section{Section 2.10. The Health Care Access Framework}

The integration and synthesis of scholarship from sociology, policy, organizational behavior, systems science, and economics, supports the proposition that supply-side attributes, inside and outside the hospital, influence hospital OAT delivery. Levesque et al. (2013) proposed an updated conceptual framework on health services access through a synthesis of work from Bashshur, Shannon, and Metzner (1971), Donabedian (1974), Salkever (1976), Aday and Andersen (1974), Penchansky and Thomas (1981), Dutton (1986), Frenk and White (1992), Haddad and Mohindra (2002), Shengelia, Murray, and Adams (2003), and Peters et al. (2008). 
Levesque et al. (2013) defined health care access as "the possibility to identify healthcare needs, to seek healthcare services, to reach the healthcare resources, to obtain or use health care services, and to actually be offered services appropriate to the needs for care" (p. 4). The outcomes of achieving access are the utilization of services and health care consequences (i.e., economic, satisfaction, and health). From this perspective, access enables people to take steps to enter into contracts with health professionals and health care facilities to obtain health care services (Levesque et al., 2013). Levesque et al. (2013) explains that the differences in access (e.g., variation, underuse) may be due to either supply-side or demand-side attributes. The supply-side attributes include five categories applied to health professionals, facilities, health delivery systems, and broader influencing social factors (e.g., health insurance policy) (Levesque et al., 2013):

- Approachability - the articulation that a service exists for treatment;

- Acceptability - the cultural and social factors for the treatment;

- Availability and accommodation - the physical and timely manner of treatment;

- Affordability - the economic capacity to spend resources for treatment; and

- Appropriateness - the fit of the needs of the patient and service.

The demand-side determinants, focused largely on the capabilities of individuals, families, and communities to access care (Levesque et al., 2013):

- Perceive-the ability for the patient to perceive the need for treatment; 
- Seek - the ability for the patient to have the capacity to choose to pursue treatment;

- Reach - the ability for the patient to physically access treatment;

- Pay - the ability for the patient to generate economic resources to pay for treatment; and

- Engage-the ability for the patient to participate in treatment decision-making.

The dissertation's focus is primarily on the supply-side determinants, and uses the Levesque et al. (2013) conceptual framework as a scaffold from which to apply the other previously described, potential contributors to hospital OAT delivery.

\section{Section 2.11. OAT Delivery Outside the Hospital}

This section describes the contemporary U.S. OAT delivery system, specifically, the OTP and non-OTP delivery settings informed by the special regulatory frameworks overseen by the DEA and SAMHSA described previously. The regulatory frameworks are "special" because they are exceptional. No other FDA-approved pharmaceutical products require separate federally mandated prescriber registration programs and separate systems of delivery (i.e., non-pharmacy based) (Priest et al., 2019).

2.11.1. Opioid treatment programs (OTP). The administration of methadone for OUD treatment is governed by the Certification of Opioid Treatment Programs, 42 Code of Federal Regulations (CFR) 8 (SAMHSA, 2015). Methadone must be administered daily under direct observation in a federally licensed OTP. There are only two delivery exceptions: 1) during hospitalization; and 2) approved take home-doses (Priest et al., 
2019). The SAMHSA regulations require OTPs to be certified, accredited, and registered with the DEA (SAMHSA, 2015). OTPs are not permitted to prescribe methadone, they are only permitted to dispense methadone; thus, most patients are required to physically attend the OTP for daily observed dosing (McCarty et al., 2018). OTPs may also administer buprenorphine, and they may offer other services, such as recovery fellowship (e.g., 12-Step), and they require patient participation in nonpharmacotherapy services such as counseling (SAMHSA, 2015). In 2016, approximately 1,500 OTPs operated in the U.S. serving 350,000 people (Alderks, 2017). Patient challenges to accessing OTPs are documented and include system-level (e.g., insurance policies, program characteristics) and patient-level barriers (e.g., information barriers) (Oliva, Maisel, Gordon, \& Harris, 2011).

2.11.2. Non-OTP delivery. At present, buprenorphine, with and without naloxone, is the only FDA approved OAT that may be prescribed for OUD treatment. Buprenorphine prescribing is only allowed by federally certified prescribers (SAMHSA, 2016). To become a certified prescriber a health professional must meet the requirements of the Drug Addiction Treatment Act of 2000, including: licensed to practice under state law; registration with the DEA; and training or certification (SAMHSA, 2016). Until recently, certified prescribers only included physicians, nurse practitioners, and physician assistants; however, with the passage of the 2018 SUPPORT for Patients and Communities Act for Patients Act, clinical nurse specialists, certified registered nurse anesthetists, and certified nurse midwifes are now eligible to prescribe 
(American Nurses Association, 2018).

After the completion of the waiver requirements, and DEA approval, a certified provider may prescribe buprenorphine for a certain number of patients depending on their clinical licensure. For physicians in the first year they may prescribe to no more than 30 patients at a time; in the second year, they may request to prescribe for up to 100 patients; and in the third year they may request to prescribe for up to 275 patients (SAMHSA, 2016). Nurse practitioners and physician assistants are limited to prescribing for 30 patients (ASAM, 2018a). Finally, in addition to having the waiver, providers who wish to administer non-oral forms of buprenorphine (e.g., implant and long-acting injectable) must also become certified by the patent-holding pharmaceutical company (SAMHSA, 2018).

In contrast with the methadone system, a variety of innovative primary care models for OAT delivery have emerged (Korthuis et al., 2017). These practice-based models include office-based opioid treatment programs, the Buprenorphine HIV Evaluation and Support Collaborative Model, the One-stop Shop Model, and the Integrated Prenatal Care and Medication Assisted Treatment Model (Korthuis et al., 2017).

\section{Section 2.12. OAT Delivery within an Integrated Health System}

In the U.S. there is a dearth of integrated health systems; thus, there is much to be learned from the VHA, which has a longstanding history of providing services for 
patients with OUD. The VHA's journey with OAT delivery is described below, including system design, formulary decisions, and building system-wide treatment capacity.

2.12.1. VHA system design. The VHA provides insurance coverage and the delivery of health services to over 9 million U.S. veterans in 170 VHA-owned and operated medical centers and 1,063 outpatient sites (U.S. Department of Veterans Affairs, 2016a). The development of the VHA SUD treatment and service delivery system began in the late in response to increased OUD prevalence in veterans returning from the Vietnam War (Wyse et al., 2018). To meet the needs of these service members, the VHA created the Alcohol and Drug Dependence Service and developed specialized OUD treatment facilities (Wyse et al., 2018). The program grew rapidly and, by the end of 1972, 44 VHA Drug Dependency Treatment Centers were operational; by 1973, more than half of VHA patients (58\%) receiving outpatient OUD services were receiving methadone (Cantor, 1974). The original VHA Drug Dependency Treatment Centers operated similarly to OTPs (Wyse et al., 2018). In the decades that followed, the number of VHA facilities offering methadone declined. By 2008, only 28 VHA medical centers had onsite OTPs, and only six of 128 centers offered offsite OTP access through community-based programs (Oliva, Harris, Trafton, \& Gordon, 2012). As of 2018, the VHA operates 32 OTPS and the number of offsite OTPS at present is unknown (Wyse et al., 2018).

2.12.2. VHA formulary. The VHA's Pharmacy Benefits Management Service approved sublingual buprenorphine as non-formulary medication in 2003, meaning that 
additional administrative approvals were necessary prior to prescribing (U.S.

Department of Veterans Affairs, 2017a). Buprenorphine use during that time was limited, and after three years of non-formulary status, in 2006 , only $3 \%$ of patients with OUD received buprenorphine (Gordon et al., 2007). In 2006, the VHA had an internal policy change and sublingual buprenorphine was added to the formulary, removing administrative prescribing barriers (Wyse et al., 2018). Since that time, buprenorphine accessibility has increased in the VHA system; by fiscal year 2011, 88\% of VHA facilities offered buprenorphine ( $n=123$ out of 140 eligible facilities) (VA Office of Inspector General, 2012).

2.12.3. VHA OAT capacity. The VHA used a variety of initiatives to build OAT capacity and increase access across the system, including: educational and quality improvement initiatives; targeted funding; and national policy and clinical guidelines (Wyse et al., 2018). In 2007, the VHA provided $\$ 300,000$ to institutions without OTPs but a high need for care (Gordon et al., 2011) and instituted a national buprenorphine consult service, the Buprenorphine in the VA Initiative (Gellad, Good, \& Shulkin, 2017). In 2008, VHA national clinical guidelines outlined the "minimum clinical requirements" to be provided in VHA facilities, which included OAT provision (Wyse et al., 2018). This was the first time in VHA history that OAT was mentioned as an OUD clinical requirement (Wyse et al., 2018). One year later, in 2009 (updated in 2015), the VHA published the Clinical Practice Guidelines for the Treatment of Substance Use Disorders, 
which recommended OAT for OUD treatment (U.S. Department of Veterans Affairs, 2017b).

In spite of all these efforts, OAT is still underused in the VHA system even in the midst of increasing need. The number of veterans with OUD increased between 2004 (n $=30,093$ ) (Oliva et al., 2013) and 2012 ( $n=48,689)$ (Finlay et al., 2016). Recent data from fiscal year 2017 reported that 54,000 VHA patients had an OUD diagnosis, 38\% of them received some pharmacotherapy (OAT or opioid antagonist), and the OAT facility delivery performance ranged from 3\% to 74\% (Finlay et al., 2018). To date, however, little is known specifically about hospital OAT care delivery processes in the VHA. Research has focused on the receipt of OAT generally, and discussions of access to OAT across the OUD care continuum have not occurred.

\section{Section 2.13. OAT Delivery within the Hospital}

This section highlights the need for hospital-based OAT delivery, how it can be feasibly and effectively delivered, and the potential delivery constraints. To make this argument, the presentation of evidence includes an overview of OUD-related hospitalization trends, a review of hospital-based OUD treatment interventions, and a synopsis of the potential external and internal supply-side attributes informing hospital OAT delivery.

2.13.1. Utilization, costs, and outcomes. This sub-section describes the OUDrelated hospitalization utilization trends (e.g., national rates), health outcomes (e.g., inhospital mortality), hospital outcomes (e.g., leaving against medical advice), and 
financial implications (e.g., elevated costs of OUD-related admissions). These analyses depict the growth of OUD related admissions to support the assertion that hospitals are an important part of the OAT delivery system.

2.13.1a. Utilization trends. Three descriptive analyses (Fingar et al., 2018; Weiss et al., 2017a; Weiss et al., 2016) examined nationwide OUD-related hospitalization trends using data from the Healthcare Cost and Utilization Project (HCUP) National Inpatient Sample (NIS). ${ }^{1}$ Between 2005 and 2014 the national rate of OUD-related hospitalizations increased 64\% (Weiss et al., 2016). The national rate of inpatient stays across U.S. states, for OUD-related admissions, averaged across 2013, 2014, and 2015, was 217 per 100,000 people, a rate second to alcohol (558 per 100,000 people), and ahead of cannabis (193 per 100,000 people) (Fingar et al., 2018). During this time period, state-level rates for opioid-related admissions were highest for Maryland (411 per 100,000 people), West Virginia (201 per 100,00 people), and Massachusetts (373 per 100,000 people) (Fingar et al., 2018).

Specific trends across the population were observed for patient gender and patient income quartile. In 2014, women had a higher rate of OUD-related inpatient stays than men in nearly $75 \%$ of jurisdictions (i.e., states and the District of Columbia) (Weiss et al., 2017a). In the same year, the OUD-related admission rates were highest for patients with the lowest income $(297$ per 100,000) and were lowest for patients with

\footnotetext{
${ }^{1}$ HCUP NIS is a database of hospital inpatient admissions from a nationally representative of community hospitals, from all payers, and uses a hospital sampling frame comprising more than 95\% of all U.S. discharges (Fingar et al., 2018; Weiss et al., 2017a; Weiss et al., 2016).
} 
the highest income (158 per 100,000) (Weiss et al., 2017b). In contrast, there was variation in the highest rates of admission based on patient age and area of residence (urban vs. rural) (Weiss et al., 2017a, 2017b).

2.13.1b. Costs. Using HCUP data, researchers estimated that OUD-related hospitalizations in the U.S. cost $\$ 15$ billion annually and that these costs increased over time (Ronan \& Herzig, 2016). Costs for OUD-related admissions and OUD-related admissions with co-infection increased between 2002 and 2012 (Ronan \& Herzig, 2016). For OUD-related admissions without an infection, charges more than tripled from $\$ 4.6$ billion in 2002 to $\$ 14.9$ billion in $2012(p<0.001)$ and for patients with a co-occurring infection the costs increased from \$190.7 million in 2002 to $\$ 700.7$ million in 2012 ( $p<$ 0.001) (Ronan \& Herzig, 2016). In 2012, the estimated total charge per OUD-related hospitalization was $\$ 28,543$ and for OUD-related hospitalization with associated infection, the cost was $\$ 107,217$ (Ronan \& Herzig, 2016). Similarly, an analysis of opioidassociated overdose admissions to the intensive care unit (ICU) between 2009 and 2015 using data from 162 hospitals in 44 states found the mean cost per ICU overdose admission increased from $\$ 58,517(2009)$ to $\$ 92,408(2015)$, a 58\% increase $(p<0.0001)$ (Stevens et al., 2017).

Costs for OUD-related hospitalizations were higher and longer than non-OUD related admissions. General hospital admissions had a mean length of stay of 4.5 days, costing $\$ 10,400$ per stay (Weiss \& Elixhauser, 2014). OUD-related hospital admissions, in comparison, were $15 \%$ longer (5.2 days) and nearly three times more expensive at 
$\$ 28,543$ per stay (Ronan \& Herzig, 2016). When patients with an OUD-related concurrent infection (e.g., endocarditis) were hospitalized, the length of stay more than tripled to 14.6 days with costs escalating more than ten times $(\$ 107,217$ per stay) (Ronan \& Herzig, 2016).

Some of these costs may be due to the acuity of services received. The use of ICU services for patients with opioid overdose increased between 2009 and 2015. Adjusting for covariates, there was a $0.5 \%$ per month increase over the study period ( $R R, 1.005$; 95\% Cl, 1.003 to 1.006; $p<0.0001$ ) (Stevens et al., 2017). On average, approximately $10 \%$ of ICU overdose patients required mechanical ventilation, $7 \%$ required noninvasive ventilation, $4 \%$ required vasopressors, and $6 \%$ required renal replacement (Stevens et al., 2017).

The distribution of costs by payer for OUD-related hospitalizations disproportionately burden public payers. An analysis of 2015 HCUP data found that $72 \%$ of OUD admissions were paid for with public funds (Medicaid 40\%; Medicare 32\%) (Weiss \& Heslin, 2018). Between 2010 and 2015, the share of opioid-related inpatient stays increased for public payers and decreased for private payers and the uninsured (Weiss \& Heslin, 2018). Ronan and Herzig (2016) also found that Medicaid was the most common payer for patients with an OUD-related diagnosis code or any OUD-related infection diagnosis code (i.e., endocarditis, osteomyelitis, septic arthritis, or epidural abscess). In 2012, Medicaid covered 37\% of all OUD-related admissions without 
infection and $43 \%$ of all admissions with OUD-related co-infections (Ronan \& Herzig, 2016).

2.13.1c. Health outcomes. Persons with OUD-related hospital admissions have a relatively low rate of in-hospital death, although the rates are increasing for persons receiving higher levels of care. In 2012, 1\% of OUD-related hospital admission patients died and $3 \%$ of OUD-related co-infection admissions patients died (Ronan \& Herzig, 2016). In the ICU, there was a significant increase in hospital deaths for opioid overdose over time: in 2009 the average mortality was 7\%; and increased to 10\% by 2015 (Stevens et al., 2017).

The rates of in-hospital mortality for persons with OUD may depend on the type of opioid used and the presence of a co-infection. An analysis of the HCUP data between 2001 and 2012 found that heroin overdose admissions were twice as likely to lead to inhospital death as compared to prescription opioid overdose admissions (Hsu, McCarthy, Stevens, \& Mukamal, 2017). In multivariate models studying OUD-related ICU admissions, the number of hospitalizations with heroin overdose each month was associated with an increase in the monthly mortality rate among ICU patients with OUDrelated overdoses (relative rate $[R R] 1.25 ; 95 \%$ confidence interval $[\mathrm{CI}][1.14,1.37] ; p<$ 0.001) and ICU admissions with an overdose during the summer were associated with lower mortality (RR, 0.87; 95\% Cl [0.78, 0.97]; $p=0.012)$ (Stevens et al., 2017). Finally, patients with an OUD-related hospitalization and an associated co-infection were more likely to die than those without infection (Ronan \& Herzig, 2016). These findings were 
observed at two time points: 2002 and 2012 ( $p<0.001$ for each comparison) (Ronan \& Herzig, 2016).

These analyses suggest that in-hospital mortality is a relatively rare event and occurs at a stable rate for aggregated OUD-related admissions. The data also illustrates that the use of heroin and the presence of a co-infection were associated with increased risk of death. What is not captured is the mortality risk to patients withdrawn from opioids or OAT upon discharge. Research in other settings found elevated mortality during the first two-weeks following release from U.S. correctional facilities. In one study, persons with drug use disorders had a three to eight times increased risk of drugrelated death (Merrall et al., 2010). No research to date has completed an assessment of overdose risk upon discharge from the hospital setting. Thus, it is possible that inhospital mortality does not fully capture death risk related to OUD-related hospitalizations.

2.13.1d. Sub-optimal and inappropriate care. For hospitalized patients suboptimal OUD care may be reflected by three indicators: 1) elevated rates of leaving against medical advice; 2) low use of OAT during admission; and 3) low OAT use in the 30 days after hospital discharge. Inappropriate care may be reflected in the increased use of short-acting opioids during and after admission.

In the 2012 HCUP data, $12 \%$ of patients admitted with an OUD-related condition left against medical advice (AMA) (Ronan \& Herzig, 2016). This rate is elevated when compared to the $1 \%$ AMA rate for all cause admissions (Stranges et al., 2009). The top 
five diagnoses with the highest relative rate of leaving AMA included patients with a primary diagnosis of: alcohol-related disorders (RR, 11.6), substance-related disorders (RR, 10.8), nonspecific chest pain (RR, 3.6), diabetes with complications ( $R R, 2.7)$, and mood disorders (RR, 1.9) (Stranges et al., 2009). High rates of leaving AMA may be a proxy measure for hospital care quality, reflecting that patient's physical and emotional needs are not being met during hospitalization.

Limited research supports the assertion that OAT initiation and continuation is a relatively rare event during OUD-related hospitalizations. In a ten-year (2004 to 2014) retrospective chart review of hospitalized patients with injection drug use-associated infective endocarditis in Boston, Massachusetts, OAT was documented in $11 \%$ of admissions ( $n=26$ ) (Rosenthal et al., 2015). Upon closer review of the 26 admissions, one patient was started on methadone, one was provided a phone number for an outpatient buprenorphine clinic, and the other 24 patients were continued on their previously initiated OAT-related treatments, none of which were arranged during their hospitalization (Rosenthal et al., 2015).

Even the occurrence of serious OUD-related sentinel events-outpatient and inpatient medical attention because of an overdose-does not dramatically increase OAT provision upon hospital discharge. A retrospective analysis assessed the association between prescription opioid overdose and post-overdose OAT provision using Medicaid claims data from 2008 to 2013 in Pennsylvania (Frazier et al., 2017). Among patients who survived a heroin overdose, there was a $1 \%$ increase in buprenorphine prescribing 
$(95 \% \mathrm{Cl}[-0.8,2.9] ; \mathrm{p}=0.27)$ and a $2 \%$ increase in admissions to methadone treatment $(95 \% \mathrm{Cl}[0.7,3.8] ; p=0.005)$ (Frazier et al., 2017). For persons with a prescription opioid overdose, the use of buprenorphine increased $1 \%(95 \% \mathrm{Cl}[0.5,2.0] ; \mathrm{p}=0.001)$ and methadone use increased $0.1 \%(95 \% \mathrm{Cl}[-0.5,0.7] ; \mathrm{p}=0.75)$ (Frazier et al., 2017). Similarly, the provision of OAT post-OUD related admission discharge for privately insured persons was also low. An analysis of claims for privately insured adults with an OUD-related diagnosis who were admitted to an acute care hospital or psychiatric hospital found low rates of OAT within the first 30 days post-discharge: just one in six patients $(17 \% ; n=6,132)$ received an FDA-approved OUD treatment medication (buprenorphine, methadone, naltrexone) and 35\% $(n=12,852)$ did not have any prescriptions filled upon discharge (Naeger, Ali, et al., 2016).

The use of short-acting opioids, instead of OAT, to manage OUD during hospitalization is not explicitly described in the literature. A retrospective cohort study of adult non-surgical hospital admissions from 286 U.S. non-federal acute care facilities found that opioid exposure in the hospital was very common (51\% of 1.1 million patient admissions). The average opioid exposure in this sample was a mean daily morphine milligram equivalent (MME) of $68 \pm 185$, and $23 \%$ of opioid exposed admissions had an MME dosage greater than or equal to 100 (Herzig, Rothberg, Cheung, Ngo, \& Marcantonio, 2014). Moreover, there was substantial variation in opioid exposure across hospitals, ranging from $5 \%$ of all admissions in a hospital to $72 \%$ of admissions (Herzig et al., 2014). 
There is research, however, describing the inappropriate use of opioids in the time period post-admission for patients with OUD (Larochelle et al., 2016; Naeger, Mutter, et al., 2016). Naeger, Mutter, et al. (2016) observed that in the first 30 days after discharge from an OUD-related hospital admission $22 \%(n=8,225)$ of patients filled a prescription for a non-OAT opioid pain medication, $14 \%$ of patients filled a prescription for a benzodiazepine $(n=5,104)$, and $7 \%$ of patients $(n=2,717)$ filled a prescription for a benzodiazepine and a non-OAT opioid. A retrospective cohort study from May 2000 to December 2012, using the Optum claims database (a large private U.S. health insurer with members in all 50 state), observed that $91 \%$ of patients with a nonfatal opioid overdose who were receiving long-term opioid therapy prior to their opioid overdose continued to receive non-OAT opioids after hospital discharge (Larochelle et al., 2016).

2.13.2. Evidence for OUD treatment. This section summarizes a limited, but growing evidence base of interventions to enhance services for patients hospitalized with OUD. This narrative review includes a summary of the single clinical trial that exists on hospital OAT delivery, a summary of published hospital practice checklists and recommendations, and the retrospective and prospective evaluations of addiction medicine consult (AMC) services.

2.13.2a. OAT initiation. A robust literature exists supporting OAT initiation and long-term use in community-based settings, including specialty addiction facilities, OTPs, and primary care clinics, but only one randomized control trial has been published 
assessing hospital-based OAT initiation (Liebschutz et al., 2014). The primary finding from this study was that participants who initiated OAT (i.e., buprenorphine) while hospitalized were more likely to engage in treatment post-discharge (Liebschutz et al., 2014). Participants $(n=145)$ were randomized into either a detoxification protocol (i.e., a five-day buprenorphine taper) or a linkage protocol (i.e., buprenorphine induction, stabilization and transition to a primary care clinic for continued) (Liebschutz et al., 2014). Buprenorphine engagement, verified by medical record, and self-reported prior 30-day use of illicit opioids, was assessed at one, three, and six months (Liebschutz et al., 2014). The linkage group was more likely to start buprenorphine ( $n=52 ; 72 \%)$ than those in the detoxification group $(n=8 ; 12 \% ; p<0.001)$. At six months, 12 linkage participants (17\%) and two detoxification participants (3\%) were still receiving buprenorphine $(p=0.007)$ (Liebschutz et al., 2014). Linkage participants reported less illicit opioid use in the 30 days prior to the six-month follow-up interview (incidence rate ratio [IRR] 0.60; $95 \% \mathrm{Cl}[0.46,0.73] ; \mathrm{p}<0.01)$ and linkage participants were more likely to report no illicit opioid use ( $n=24 ; 38 \%$ vs. $n=5 ; 9 \%$ (Liebschutz et al., 2014). The estimated OAT rate among linkage participants was almost 2.4 times higher throughout the six-month study period (IRR, 2.44; 95\% Cl [1.99, 3.36]; $\mathrm{p}<0.01$ ) (Liebschutz et al., 2014). Six participants died over the course of the study, and only one death was related to drug overdose (Liebschutz et al., 2014).

2.13.2b. OUD treatment practice and delivery recommendations. There is a growing collection of published narrative reviews and clinical checklists on service 
delivery for hospitalized patients with OUD and other SUDs (Noska et al., 2015; Sharma et al., 2017; Thakarar et al., 2016; Theisen-Toupal et al., 2017; Weinstein et al., 2018) and federal guidance documents (SAMHSA, Institute of Medicine Committee on Federal Regulation of Methadone, 1995; 2018). The reviews and checklists suggest the implementation of a variety of interventions for patients (i.e., psychosocial, medical, and harm reduction) and for systems (e.g., AMC services and education on addiction and stigma for health professionals), summarized in Table 2.1. The federal guidance documents recommend hospital OAT delivery. 
Table 2.1. Summary of Narrative Review Recommendations for Hospital-Based Treatment of OUD

\begin{tabular}{|c|c|c|c|c|}
\hline \multirow[t]{2}{*}{ Authors } & \multicolumn{3}{|c|}{ Patient-Level Interventions } & \multirow[t]{2}{*}{ System-Level Interventions } \\
\hline & Psychosocial & Medical & Harm Reduction & \\
\hline $\begin{array}{l}\text { Theisen- } \\
\text { Toupal et } \\
\text { al. (2017) }\end{array}$ & $\begin{array}{l}\text { - Involve social work } \\
\text { - Involve case } \\
\text { management }\end{array}$ & $\begin{array}{l}\text { - Manage opioid withdrawal } \\
\text { - Initiate OAT or antagonist therapy } \\
\text { - Treatment of special populations } \\
\text { (e.g., acute pain/breastfeeding) } \\
\text { - Discharge checklist }\end{array}$ & $\begin{array}{l}\text { - Provide opioid overdose } \\
\text { education } \\
\text { - Provide safer injection } \\
\text { education }\end{array}$ & $\begin{array}{l}\text { Partnerships between hospitals and } \\
\text { outpatient addiction treatment } \\
\text { facilities }\end{array}$ \\
\hline $\begin{array}{l}\text { Thakarar } \\
\text { et al. } \\
(2016)\end{array}$ & $\begin{array}{l}\text { - Collaborate with } \\
\text { mental health } \\
\text { service providers } \\
\text { - Provide case } \\
\text { management for } \\
\text { supportive services }\end{array}$ & $\begin{array}{l}\text { - Conduct addiction history } \\
\text { - Assess treatment readiness } \\
\text { - Provide treatment options (OAT or } \\
\text { antagonist therapy) }\end{array}$ & $\begin{array}{l}\text { - Provide opioid overdose } \\
\text { prevention } \\
\text { - Provide infection prevention }\end{array}$ & - Implement the checklist \\
\hline $\begin{array}{l}\text { Sharma et } \\
\text { al. (2017) }\end{array}$ & & $\begin{array}{l}\text { Future Research Directions } \\
\text { - Optimal use of peripherally } \\
\text { inserted central venous catheters } \\
\text { - Treatment contracts } \\
\text { - Inpatient supervised injection } \\
\text { services }\end{array}$ & $\begin{array}{l}\text { Implementation Research } \\
\text { - Provide injectable } \\
\text { diacetylmorphine } \\
\text { - Provide sterile drug use } \\
\text { equipment. } \\
\text { - Future harm reduction }\end{array}$ & $\begin{array}{l}\text { For Immediate Implementation } \\
\text { - Establishment of AMC service } \\
\text { - Education for health professionals on } \\
\text { stigma and addiction } \\
\text { Implementation Research } \\
\text { - Care retention and continuity at } \\
\text { discharge }\end{array}$ \\
\hline $\begin{array}{l}\text { Noska et } \\
\text { al. (2015) }\end{array}$ & & $\begin{array}{l}\text { - Establish OUD diagnosis } \\
\text { - Manage opioid withdrawal } \\
\text { - Initiate OAT and linkage to care }\end{array}$ & & $\begin{array}{l}\text { - Educate providers on federal, state, } \\
\text { and hospital policies } \\
\text { - Educate providers on medications } \\
\text { available to treat opioid withdrawal }\end{array}$ \\
\hline $\begin{array}{l}\text { Weinstein } \\
\text { et al. } \\
\text { (2018) }\end{array}$ & - Referral and linkage & $\begin{array}{l}\text { - } \text { Addiction history } \\
\text { - Physical examination/laboratory } \\
\text { tests } \\
\text { - Withdrawal management } \\
\text { - Long-term medication titration }\end{array}$ & $\begin{array}{l}\text { - Provide opioid overdose } \\
\text { education } \\
\text { - Provide safer injection } \\
\text { education }\end{array}$ & - Establishment of AMC service \\
\hline
\end{tabular}


Federal guidance documents from SAMHSA (SAMHSA, 2018) and the Institute of Medicine (Institute of Medicine Committee on Federal Regulation of Methadone, 1995) describe the most effective approaches to hospital OAT delivery. The SAMHSA document dedicated an entire chapter to the "Medical Management of Patients Taking OUD Medications in Hospital Settings" (SAMHSA, 2018, pp. 3-99). The recommendations for effective care for patients with OUD who are hospitalized include balancing OUD pharmacotherapy with other medical concerns, careful management after discharge, and seamless transfer to OAT upon discharge (e.g., either OTP or non-OTP) (SAMHSA, 2018). The document included clinical management guidance for initiating and continuing OAT during admission (SAMHSA, 2018).

The 1995 Institute of Medicine provided six recommendations on how to care for patients already on methadone during an inpatient admission (Institute of Medicine Committee on Federal Regulation of Methadone, 1995):

- Hospital staff should notify the OTP, confirm enrollment, and verify methadone dose, and time and date of last dose;

- Hospital staff should ensure the continuity of methadone pharmacotherapy through the hospital's pharmacy or make arrangement for its supply through the OTP in the event that the hospital does not stock methadone;

- Hospital staff should be prohibited from reducing or denying the methadone dose; 
- Any changes in methadone dose should be made only after consultation with the OTP physician and with the informed consent of the patient;

- Before discharge, the hospital staff should notify the OTP of the time and amount of last dose of methadone to ensure that outpatient treatment resumes without interruption; and

- If patients are discharged from acute care to continuing care facilities, arrangements for continued provision of methadone should be part of the discharge plan.

The committee also recommended that rule-making agencies modify the federal regulations to allow hospitals to continue to provide methadone if the patient is unable to get into an OTP or if they need to complete a methadone taper (Institute of Medicine Committee on Federal Regulation of Methadone, 1995).

2.13.2c. The AMC services. A growing literature base suggests that the AMC service is an effective health services delivery intervention. The literature on AMCs, however, should be interpreted conservatively because it is comprised of non-blinded and non-randomized prospective (Trowbridge et al., 2017; Wakeman et al., 2017) and retrospective studies (Nordeck et al., 2018; Suzuki, 2016).

Two prospective AMC service evaluation studies found that patients who received $A M C$ services had an increased number of drug free days post-discharge, compared with those who did not (Wakeman et al., 2017), and that hospital OAT initiation was possible (Trowbridge et al., 2017). 
A prospective quasi-experimental evaluation at an urban academic hospital in the northeast compared 30-day post-discharge outcomes between hospitalized participants who received or did not receive an AMC service (Wakeman et al., 2017). The AMC service intervention included a multidisciplinary team offering pharmacotherapy initiation, motivational counseling, treatment planning, and direct linkage to external addiction treatment resources (Wakeman et al., 2017). The majority of patients (61\%) had an OUD-related diagnosis (Wakeman et al., 2017). In this nonrandomized intervention, patients who received an addiction consult, compared to those who did not, had a mean drug Addiction Severity Index decrease pre-and post $(p=$ 0.003 ) and increased days of abstinence at 30-day follow-up (+12.7 days vs. $+5.6 ; p<$ 0.001) (Wakeman et al., 2017).

A prospective evaluation at Boston Medical Center assessed the process and care delivery measures of an AMC service (Trowbridge et al., 2017). Over a 26-week period the AMC service completed 337 of 367 requested consults for 319 unique patients; of those $78 \%$ were for persons with an OUD $(n=264)$ (Trowbridge et al., 2017). For those with OUD, methadone was initiated in $27 \%$ of patients $(n=70)$ and buprenorphine was initiated in $15 \%$ of patients $(n=40)$, with $42 \%$ of patients with OUD initiated on OAT (Trowbridge et al., 2017). Of those initiated on methadone, $76 \%$ were linked to methadone clinics and for buprenorphine, $49 \%$ were linked to a clinic (Trowbridge et al., 2017). Researchers observed that OAT initiation was feasible in the hospital setting; however, effectively linking and retaining patients in post-discharge 
addiction care was challenging, warranting future program development (Trowbridge et al., 2017).

The two retrospective AMC service studies found that most patients were interested in receiving AMC services (Nordeck et al., 2018; Suzuki, 2016). The University of Maryland Medical Center SUD consultation liaison service-operational for over three decades with an interprofessional team of a psychiatrist director, two part-time addiction-boarded psychiatrists, a licensed addiction counselor, a licensed social worker, nurses, and medical trainees - reported service delivery for 267 patients (Nordeck et al., 2018). A majority (57\%) had a current OUD diagnosis $(n=152)$ and OAT with buprenorphine or methadone was initiated for $14 \%$. Within patients with OUD who were referred to an affiliated program during hospitalization $(n=45), 42 \%$ of patients who initiated hospital OAT kept their intake appointment (Nordeck et al., 2018). A final observation was that only $10 \%$ of patients expressed no interest in treatment or refused a formal consultation (Nordeck et al., 2018); indicating, that hospitalization was a reachable moment.

A retrospective chart review examined the health records for patients with OUDrelated admissions and infective endocarditis who received AMC services between May 2013 and July 2015 (Suzuki, 2016). Twenty-nine patients were admitted for the treatment of infective endocarditis secondary to associated intravenous drug and had a request for an addiction psychiatry consultation (Suzuki, 2016). All patients were evaluated to initiate OAT, efforts were made to find community-based prescribers to 
continue treatment, and patients were encouraged to have an initial appointment established prior to discharge (Suzuki, 2016). The majority (72\%) of hospitalized patients in this cohort had previous experience with OAT. Almost two-thirds (62\%) of patients accepted and received OAT $(n=18)$, of those, half initiated buprenorphine and half initiated methadone maintenance (Suzuki, 2016).

2.12.3. External supply-side attributes. External influencers on hospital OAT delivery are essentially unstudied. This section is a summary of potential external attributes that may facilitate or impede hospital OAT delivery, such as third-party payer policies (e.g., pharmacy benefit management strategies) and federal regulations.

2.12.3a. Payer policies. Payer policies may contribute to limited hospital OAT access, although most of hospital billing occurs through bundled payments. Limitations imposed by third-party payers on OAT in the outpatient setting may constrain services delivered in the hospital setting, particularly when starting people on OAT during hospitalization (Hassamal et al., 2017).

OAT insurance barriers are well-described for public and private third-party payers. Medicaid, the most common payer for OUD-related hospitalizations (Weiss \& Heslin, 2018) imposes a variety of pharmacy benefit management strategies for OAT (i.e., prior authorization, quantity limits, and lifetime limits). In an analysis of Medicaid Preferred Drug Lists (2011 to 2013), 31 State Medicaid agencies covered methadone and all 50 Medicaid agencies covered buprenorphine (SAMHSA, 2014). However, 48 Medicaid programs required prior authorization for buprenorphine and 21 programs 
required evidence that the patient was receiving psychosocial addiction services (SAMHSA, 2014). Further, 34 Medicaid programs had buprenorphine quantity limits and some states had lifetime treatment limits. Four jurisdictions (District of Columbia, Illinois, Michigan, and Washington) established a one-year limit, six states (Arkansas, Maine, Mississippi, Montana, Virginia, and Wyoming) established a two-year treatment limit, and one state (Utah) established a three-year treatment limit (SAMHSA, 2014).

Like Medicaid, Medicare coverage of OAT is restrictive. Methadone was not an allowable service until the recent passage of the 2018 SUPPORT for Patients and Communities Act, and coverage restrictions for buprenorphine are common (Hartung et al., 2019). From 2007 to 2018, Medicare coverage for Suboxone ${ }^{\circledR}$ and Subutex ${ }^{\circledR}$ branded buprenorphine/naloxone tablets and films declined by $25 \%$, going from $100 \%$ plan coverage $(n=3,281)$ to $74 \%(n=2,873)$ (Hartung et al., 2019). Moreover, among plans offering coverage, prior authorization requirements for branded buprenorphine/ naloxone increased from $16 \%$ in 2007 to $58 \%$ in 2018 . In 2018 , moreover, $57 \%$ of plans required prior authorization for generic buprenorphine/naloxone sublingual tablets (Hartung et al., 2019). The proportion of plans covering any buprenorphine product without any restriction declined from $89 \%$ to $35 \%$ in this same time period (Hartung et al., 2019).

Private payers, follow public payer practice, limiting OAT access through pharmacy benefit management strategies (Reif et al., 2016). In an analysis of 8,427 plans in the 2010 benefit year, $100 \%$ of the plans had buprenorphine coverage but $40 \%$ had 
prior authorization requirements, and only $41 \%$ of plans covered methadone for OUD (Reif et al., 2016).

2.12.3b. Federal regulations. It is possible that confusion about federal regulations may contribute to limited hospital OAT access. In 2002, the DEA director addressed provider uncertainty in a letter reminding hospital providers that it is legal to administer OAT during hospitalization (Nagel, 2002). The regulations in 21 CFR 1306.07 sub-section (b) state:

(b): Nothing in this section shall prohibit a physician who is not specifically registered to conduct a narcotic treatment program from administering (but not prescribing) narcotic drugs to a person for the purpose of relieving acute withdrawal symptoms when necessary while arrangements are being made for referral for treatment. Not more than one day's medication may be administered to the person or for the person's use at one time. Such emergency treatment may be carried out for not more than three days and may not be renewed or extended. (DEA, 2005)

These regulations, known as the three-day rule or 72-hour rule, allow hospital providers without a buprenorphine waiver or OTP facility designation, to administer OAT for 72 hours for patients who have a primary diagnosis of opioid withdrawal. If a patient is discharged with a buprenorphine bridge script, a provider with a buprenorphine waiver must issue that prescription. Further, if the provider has a buprenorphine waiver, they could continue to administer buprenorphine for more than 72 hours as needed.

The second part of the rule allows for unconstrained OAT or other non-FDA opioid administration for hospital providers without a buprenorphine waiver or OTP facility designation for patients who are hospitalized with a non-withdrawal primary diagnosis (e.g., opioid withdrawal in the setting of a hospital admission for pneumonia): 
(c): This section is not intended to impose any limitations on a physician or authorized hospital staff to administer or dispense narcotic drugs in a hospital to maintain or detoxify a person as an incidental adjunct to medical or surgical treatment of conditions other than addiction, or to administer or dispense narcotic drugs to persons with intractable pain in which no relief or cure is possible or none has been found after reasonable efforts. (DEA, 2005)

This policy is nuanced, but sub-sections b and c in 21 CFR 1306.07 explicitly state that federal law allows for the continuation and initiation of OAT in the hospital setting within certain parameters. It is possible that this policy constrains hospital OAT delivery, Hassamal et al. (2017) described provider concerns about DEA audits. Other federal policies that may constrain hospital OAT delivery include the buprenorphine patient panel limits and the administrative requirements of the special regulatory system, which has been described elsewhere in this dissertation (Sections 2.5 and 2.11).

2.13.4. Internal supply-side attributes. A limited literature (Hassamal et al., 2017; Velez et al., 2017) characterizes the constraints within the hospital environment on OAT delivery. This section is a summary of this literature and includes proposed internal attributes that may facilitate or impede hospital OAT delivery including hospital providers and hospital structures.

2.13.4a. Hospital providers. Hassamal et al. (2017), through a clinical case study and narrative literature review, describe potential OAT delivery barriers imposed by hospital providers, specifically, provider knowledge deficits (e.g., concerns about OAT misuse, lack or training), and stigmatizing behavior by providers towards patients with OUD. Further, from the patient perspective, qualitative research suggests that patients 
would benefit from the inclusion of other non-traditional types of providers in the hospital setting, such as peer support providers (Velez et al., 2017).

2.13.4b. Hospital attributes. Hassamal et al. (2017) also discussed how hospital organizational attributes likely inhibit hospital OAT delivery including concerns about outside perceptions (e.g., worried about attracting too many OUD patients), a lack of support staff and institutional support, frequent laboratory testing, and inadequate referral networks. Patients described hospitalizations as a limited context for treating addiction-related issues because hospitalizations generally do not address life stressors, trauma, or basic needs (Velez et al., 2017).

Other internal hospital delivery structures that may influence hospital OAT delivery are the hospital formulary governance body (the Pharmacy \& Therapeutics Committee $[\mathrm{P} \& T]$ ) and other OAT-related hospital policies, procedures, and guidelines. The P\&T committee manages the hospital formulary system (American Society of Health-System Pharmacists [ASHSP], 2008). Formulary system management is a process for making hospital-wide pharmaceutical and therapeutic policies (ASHP, 2000). A formulary - a document created and maintained by the P\&T committee-includes medications, devices, medication-use policies, decision support tools, ancillary drug information, and organizational guidelines (ASHP, 2008). P\&T committees include clinicians (e.g., physicians, pharmacists, nurses) and hospital administrators. The committee's decision-making authority is generally outlined in organizational bylaws, medical staff rules, and regulations specific to each hospital or health system (ASHP, 
2008). Formulary decisions should include the consideration of patient care and an unbiased literature review (ASHP, 2008), including the assessments of the pharmaceutical evidence-base and pharmaco-economic literature (M. Hoffmann, 2013).

The existence of P\&T committees is nearly universal in hospitals around the world (Durán-García, Santos-Ramos, Puigventos-Latorre, \& Ortega, 2011). In the U.S., the Joint Commission, the primary hospital accrediting body, requires a hospital P\&T committee (Balu, Connor, \& Vogenberg, 2004). P\&T committee outputs may include policy statements, procedures, and guidelines that provide guidance on service delivery within the hospital (O'Donnell \& Vogenberg, 2012). These documents are commonly created because of accreditation requirements and, to avoid litigation, align hospital services with the standard of practice for reasonable and prudent hospital pharmacists (O’Donnell \& Vogenberg, 2012).

2.13.5. Summary. Interest is growing, and the literature base is expanding, to support the assertion that hospital OAT delivery is needed, feasible, and effective. OUDrelated hospital admissions and costs are increasing. Communicating and framing hospital OAT delivery as a supply-side issue may help motivate hospitals and health services administrators to support hospital OAT delivery because people with OUD are publicly insured or uninsured. Keeping patients with OUD from readmission by providing evidence-based OAT during and after hospitalization could benefit the hospital financially in the long-term. 
Although, comparative-effectiveness research examining the best way to design AMC services has not yet occurred, it seems likely that AMC services are feasible to implement and effective in providing evidence-based services. Further, it is possible that unexplored internal hospital attributes may influence hospital OAT delivery such as the OAT-related decisions made by members of the P\&T committee or the adoption of hospital guidelines.

There is limited evidence to suggest that elements within the hospital and outside the hospital contribute to hospital OAT delivery. Outside the hospital potential external attributes that may impede hospital OAT delivery are third-party payer policies (e.g., pharmacy benefit management strategies) and federal regulations, specific to hospital delivery, and more broadly, the special OAT regulatory system. Within the hospital potential internal attributes informing hospital OAT delivery include the behavior and training of hospital providers and hospital structures. This dissertation aims to explore these issues and other unidentified facilitating and constraining elements.

\section{Section 2.14. Development of the Conceptual Framework: Synthesis of the Literature}

This section synthesizes the reviewed literature to support the underlying premise of this dissertation that OAT provision is influenced by a complex array of dynamically related supply-side factors, external and internal to the hospital (see Table 2.2). 
Table 2.2. Literature Reviewed

\begin{tabular}{|c|c|}
\hline $\begin{array}{c}\text { Scholarship } \\
\text { Domain }\end{array}$ & Theory, Model, Framework \\
\hline $\begin{array}{l}\text { Medical } \\
\text { Sociology }\end{array}$ & $\begin{array}{l}\text { - Social Construction Theory (Bacchi, 2012; Brown, 1995; Reinarman \& Granfield, } \\
\text { 2014; Rosenberg, 1989) } \\
\text { - Moral Model (Boyd et al., 2016; Reinarman \& Granfield, 2014; Valverde, 1998) } \\
\text { - Disease Model (Boyd et al., 2016; Reinarman \& Granfield, 2014; J. Schneider, } \\
\text { 2014) } \\
\text { - Multi Model (Kovac, 2014; Reinarman \& Granfield, 2014) }\end{array}$ \\
\hline Policy Process & $\begin{array}{l}\text { - The Social Construction Framework (Ingram \& Schneider, 1990, 1991; A. L. } \\
\text { Schneider \& Ingram, 1988, 1993) } \\
\text { - Policy Feedback Theory (Pierson, 1993; Skocpol, 1992) } \\
\text { - Comparative Drug Policy Analytic Framework (MacCoun et al., 1993) } \\
\text { - Drug Policy Regimes (Babor et al., 2010) }\end{array}$ \\
\hline $\begin{array}{l}\text { Organization } \\
\text { Behavior }\end{array}$ & $\begin{array}{l}\text { Elements of Leavitt's Diamond (Berger \& Luckmann, 1966; Chaffee, 1985; } \\
\text { Chandler, 1962; Davis, 1949; Galbraith, 1982; Giddens, 1979; Hillman et al., 2009; } \\
\text { Homans, 1950; Kinicki \& Kreitner, 2009; Leavitt, 1962; Ostroff et al., 2003; Pfeffer } \\
\text { \& Salancik, 1978; Scott, 2003; Weick, 1995) } \\
\text { - Institutional Theory (DiMaggio \& Powell, 1983) }\end{array}$ \\
\hline System Science & $\begin{array}{l}\text { - Sociotechnical Organizations (DiMaggio \& Powell, 1991; Katz \& Kahn, 1978; Scott, } \\
\text { 2003; Scott \& Meyer, 1991; Thompson, 1967) } \\
\text { - Multilevel Attributes (Andersen et al., 2013; Basole \& Rouse, 2008; Covaleski et } \\
\text { al., 1993; Fanjiang et al., 2005; Ferlie \& Shortell, 2001; Goodrick \& Salancik, 1996; } \\
\text { Levesque et al., 2013; Lipsitz, 2012; Perrow, 1986; Ruef \& Scott, 1998; Sallis et al., } \\
\text { 2015) } \\
\text { Complex Adaptive System Theory (Lipsitz, 2012; Plsek \& Greenhalgh, 2001) }\end{array}$ \\
\hline Economics & $\begin{array}{l}\text { - Agency Theory (Alchian \& Demsetz, 1972; Eisenhardt, 1989; Jensen \& Meckling, } \\
\text { 1976; Perrow, 1986; Zeckhauser \& Pratt, 1985) } \\
\text { - Neo-Classical Economic Principles (Feldstein, 2005; Friedman, 2002; Perrow, 1986) } \\
\text { - Transaction Cost Theory (Williamson, 1979, 1985, 1994; Williamson \& Ouchi, } \\
\text { 1981) } \\
\text { - Rational Actor Model (Allison \& Zelikow, 1999; Simon, 1985) }\end{array}$ \\
\hline $\begin{array}{l}\text { Health Services } \\
\text { Delivery }\end{array}$ & - Health Care Access Framework (Levesque et al., 2013) \\
\hline
\end{tabular}

2.14.1. Study principles. There are four foundational principles that support the study rationale and the development of the conceptual framework. First, the social construction (Brown, 1995; Rosenberg, 1989) of opioids and OUD is a meta-influencer of external and internal environmental attributes. Second, a hospital is a sociotechnical (DiMaggio \& Powell, 1991; Katz \& Kahn, 1978; Scott \& Meyer, 1991; Thompson, 1967), complex adaptive (Plsek \& Greenhalgh, 2001), economic production system that exists as a functional unit (Lipsitz, 2012) within the nested context of a broader environment 
(Basole \& Rouse, 2008; Ferlie \& Shortell, 2001; Perrow, 1986; Sallis et al., 2015). Third, an inherent property of this type of system is the influence of external and internal environmental factors (Katz \& Kahn, 1978; Plsek \& Greenhalgh, 2001; Scott, 2003). The environment, inside and outside the hospital, includes the physical, technological, cultural, and social milieu (Scott \& Davis, 2015), and policy, which is frequently both a product and an influencer of social phenomena (Ingram \& Schneider, 1990, 1991; MacCoun et al., 1993; Pierson, 1993; A. L. Schneider \& Ingram, 1988, 1993; Skocpol, 1992). Fourth, hospitals are considered to be rational unitary decision-making bodies that select actions with the highest payoff after ranking and understanding the consequences (Allison \& Zelikow, 1999; Simon, 1985). This perspective suggests a hospital's decision to provide OAT reflects strategic goals, organizational objectives, and a value-maximizing activity (Allison \& Zelikow, 1999; Simon, 1985). The rational actor model application is limited in this context because within the hospital environment there are sub-systems of internal stakeholders (e.g., providers, staff, patients) and external stakeholders (e.g., payers, regulatory bodies, community, government, industry); thus, the ability of a hospital to behave strategically to produce valuemaximizing decisions is in constant tension with the values and needs of stakeholders.

2.14.2. Supply-side focus. The study's focus on the supply-side versus demandside is because demand-side factors may have a smaller contribution for this specific care delivery setting: a) hospitalized persons with OUD will physiologically experience the need for opioids and seek opioids to prevent withdrawal symptoms; b) the patient 
can receive OAT in the hospital only if the hospital provides it; and c) OUD patients generally do not choose which hospital they are admitted to.

2.14.3. Conceptual framework. The four foundational study principles and the literature review provide the scaffolding of the study's conceptual framework and how the study is operationalized. The conceptual framework (see Figure 2.1) depicts the interactions, contributions, and feedback among the nested layers of supply-side attributes, inside and outside the hospital, on hospital service delivery, outcomes, and demand-side attributes. The elements existing within each nested layer dynamically interact across levels shaping and modifying OAT service delivery over time. The culmination of these complex interactions results in hospital OAT service delivery, and the subsequent clinical and utilization outcomes. The clinical and utilization outcomes, in turn, influence the nested supply-side attributes through feedback. The categories are interdependent and overlapping in nature.

2.14.3a. Defining hospital OAT delivery. There are few clinical scenarios that would prohibit OAT initiation or continuation for the treatment of OUD in the hospital. Clinical experts and the peer-reviewed literature described potential OAT care delivery scenarios as: 1) starting OAT in the hospital; 2) continuing OAT in the hospital; and 3) linking patients to OAT upon discharge. Part of the exploratory component of this study required further defining these care delivery scenarios, and the discovery of others.

2.14.3b. Internal hospital elements. Categories of potential hospital supply-side attributes include: 1) physical structure (e.g., urban vs. rural); 2) social structures (e.g. 
organizational culture); and 3) technology and resources (e.g., polices, regulations, and procedure; and resources and staffing).

A hospital's physical location may affect OAT delivery. Physical location may mediate hospital characteristics and processes such as hours of operation, the ability of the hospital to match the needs of the patient, and the availability of treatment (Levesque et al., 2013). Elements within the proximal physical environment, such as the urbanicity or rurality, or being located in an area with elevated or lower opioid overdose, or the proximity to other hospitals providing addiction related services, could also influence hospital OAT delivery (DiMaggio \& Powell, 1983). Other hospital attributes such as size and status as a teaching hospital affect quality of care (Keeler et al., 1992).

Hospital social structures could influence OAT delivery (e.g., the organizational values, norms, and role expectations) (Davis, 1949; Scott, 2003). Social structures may also inform hospital resource allocation. Deciding to invest, or not invest, in addictionrelated treatment technologies within the hospital is based, in part, by the alignment of this investment with current organizational strategic goals, objectives, and the determination that this is a value-maximizing activity for the hospital (Allison \& Zelikow, 1999; Simon, 1985). Another component of social structures, influencing care delivery, is the shared cultural-cognitive elements among hospital staff (Berger \& Luckmann, 1966; Scott, 2003; Weick, 1995). For example, a hospital that collectively believes in the social construction of addiction as a moral or criminal issue may be more likely to hire health 
professionals with similar beliefs, and could be less likely to provide evidence-based OAT. In contrast, a hospital with a shared philosophy across staff that addiction is a brain disease may be more likely to hire professionals with shared beliefs, and increase the likelihood of providing evidence-based OAT.

Differences in organizational structures of practice may also change delivery (De Jong, 2008). Hospital technologies, in this context, include dedicated resources, staffing, and internal policies and regulations. The existence, or lack thereof, of these attributes could influence hospital OAT delivery. Staffing technologies of interest include the existence of specific staffing structures and models of care for patients with OUD (e.g., AMC services to deliver care for patients with OUD), the availability of addiction trained specialists (e.g., board-certified addiction medicine physicians), and graduate medical education training programs (e.g., addiction medicine fellowships). Other internal resources that may influence care include access to OAT on the hospital formulary and the existence of hospital policies that either enhance or constrain this practice.

2.14.3c. Elements outside the hospital. Domains of potential hospital supplyside attributes external to the hospital include: 1 ) social structures; 2 ) the influence of other hospitals; 3) polices and regulations; and 4) economic factors.

The cultural and social contexts outside the hospital environment are also likely to influence hospital OAT delivery through communities' professional values, norms, culture, and gender of the health professionals (Levesque et al., 2013; Scott, 2003). The training and socialization of health professionals largely occurs, at least at the start of 
employment, from outside the employing hospital (Scott \& Davis, 2015). Health care professionals who trained during in a different era may not know that hospital OAT delivery is evidence-based practice or that only treating withdrawal symptoms is not sufficient care. In contrast, a hospital staffed with health care professionals who are knowledgeable about OUD treatment may be more likely to provide hospital OAT. It is also likely that internal hospital cultural-cognitive elements (Berger \& Luckmann, 1966; Scott, 2003; Weick, 1995), such as how OUD is conceptualized (moral model vs. brain disease model), are informed by the external environment either through public discourse (e.g., the media) and values, or the narratives espoused by external health professional training programs or organizations (e.g., the Joint Commission). Informal and formal coercive isomorphic pressures from other organizations may influence hospital OAT delivery (e.g., the government, influential organizations) (DiMaggio \& Powell, 1983). Other potentially influential elements in the external environment (e.g., coercive policies) drive isomorphic hospital behavior (DiMaggio \& Powell, 1983). Moreover, institutional theory (DiMaggio \& Powell, 1983) posits that hospitals attempt to mimic each other; thus, hospital OAT delivery could become acceptable practice through this process. The proximity to other hospitals is also relevant because few organizations create their own technologies (Scott \& Davis, 2015). Further, other organizations such as third-party contract enforcers (North, 1990; Zeckhauser \& Pratt, 1985) could influence hospital OAT delivery. Third-party contract enforcers could be patient-derived (e.g., addiction advocacy groups), non-governmental 
regulatory bodies (e.g., the Joint Commission), governmental regulatory bodies (e.g., CMS), local and state regulatory bodies (e.g., licensing boards), and professional associations (e.g., ASAM). These groups could influence hospital behavior because they bring their own values and preferences to the contractual relationship (North, 1990; Zeckhauser \& Pratt, 1985).

External polices and regulations that affect treatment access through planning, financing, and monitoring of addiction services, as well as the development of the professional workforce, are likely to influence hospital OAT delivery (Klingemann et al., 1993; Klingemann \& Klingemann, 1999). Influential policies include federal treatment regulations that govern allowable treatment in the hospital setting (e.g., the 72-hour rule), or more broadly the special regulatory framework dictating OAT delivery (e.g., OTPs).

Beyond federal regulations, policies created by non-governmental organizations could influence care. Organizations such as the Joint Commission may enhance or deter hospital OAT delivery through the creation of policies. In 2008, the Joint Commission received funding to develop, specify, and test performance measures addressing alcohol screening and pharmacotherapy for persons with SUDs, including OUD (Joint Commission, 2015). The Joint Commission has the authority to mandate the inclusion of these measures or the requirement of addiction trained staff as part of hospital accreditation. At present, there is a lack of external coercive policies and pressures from 
external organizations to mandate hospitals to deliver OAT (e.g., the Joint Commission mandating delivery of OAT in the hospital setting).

Another important policy domain is the third-party payer coverage policies that increase barriers to OAT access in the community setting and that potentially constrain hospital care. Public and private payers commonly impose pharmacy benefit management strategies on OAT and, as is the case for methadone, more commonly do not provide reimbursement (Hartung et al., 2019; Reif et al., 2016; SAMHSA, 2014).

External market forces may also influence hospital OAT delivery. Interestingly, from a supply-demand perspective, OAT is a service that promotes the best value for the patient (i.e., the demand) but it is still largely unavailable in the hospital setting (i.e. the supply). A mismatch between supply and demand means that OAT is an economically inefficient good because neither the supplier (e.g., the hospital) nor the demander (e.g., the patient) fully benefits. This discrepancy may be due to hospital reliance on contracts with external third-party payers that do not financially reward hospitals for OAT delivery.

2.14.4. Summary. The final product of this chapter is the conceptual framework (see Figure 2.1), which depicts the complex nature of hospital OAT delivery between the supply-side (i.e., the hospital, the broader environment) and the demand-side (i.e., patient demographics). The conceptual framework supported the study's rationale, design, analyses, and conclusions. 


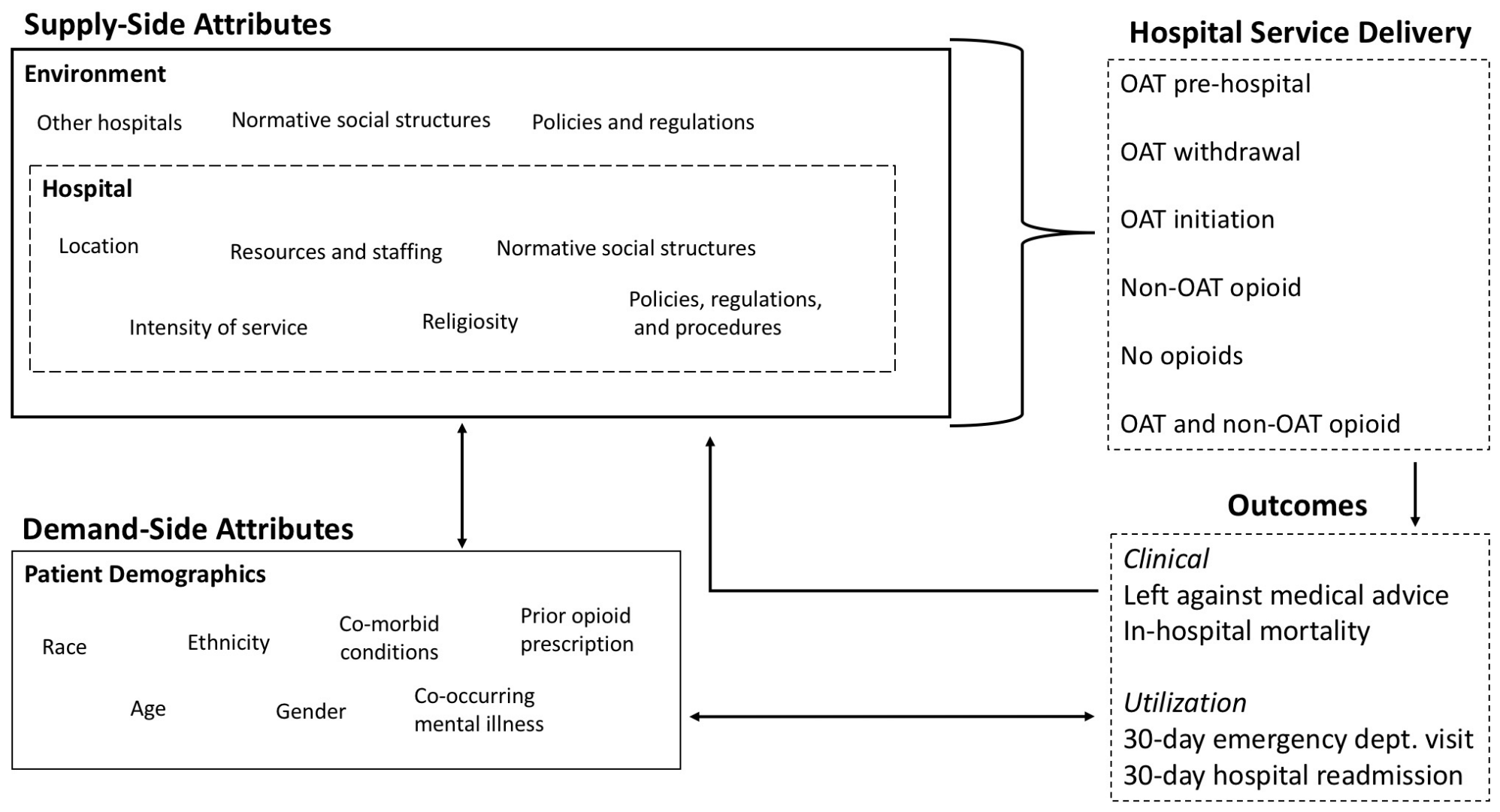

Figure 2.1. Original Conceptual Framework 


\section{Section 2.15. Conclusion}

In an era of increased OUD prevalence and care delivery in the hospital, there is an urgency to study and improve care delivery in this setting as part of the broader pathway for persons with OUD. The literature review described, summarized, and synthesized an interdisciplinary body of literature to support the research on hospital OAT delivery, and how this phenomenon is likely influenced by complex interrelated internal and external attributes. In the next chapter, Chapter 3, the study methodology is described. 


\section{Chapter 3: Methods}

Parts of this chapter were published in a peer-reviewed journal: Priest, K.C., \& McCarty, D. (2018). Role of the hospital in the 21st Century opioid overdose epidemic: The addiction medicine consult service. Journal of Addiction Medicine, Advance online publication. doi: 10.1097/ADM.0000000000000496.

Chapter 3 describes the research approach used for exploring how supply-side attributes are associated with hospital opioid agonist therapy (OAT) delivery for patients with opioid use disorder (OUD). Complex social phenomena, such as health services delivery, required a mixed methods approach, using both qualitative and quantitative data and analyses to illuminate relationships and balance methodologic limitations. The chapter is organized in six sections:

1. Section 3.1 restates and operationalizes the elements within the core research question.

2. Section 3.2 outlines the three specific aims, the 16 research sub-questions, and study design.

3. Section 3.3 justifies the research approach and outlines the study assumptions.

4. Section 3.4 provides an overview of the human subjects protections.

5. Section 3.5 describes the qualitative analytic approach for Aim 1.

6. Section 3.6 describes the quantitative analytic approach for Aims 2 and 3.

7. Section 3.7 presents the chapter's conclusions. 


\section{Section 3.1. Research Question}

The interdisciplinary theoretical literature review and the Levesque Health Care Access Framework (Levesque et al., 2013) guided the creation of the study's core research question: How do supply-side attributes influence hospital OAT delivery, health outcomes, and health services utilization for persons hospitalized with OUD?

Supply-side attributes refer to the contextual elements inside and outside a hospital that may be associated with hospital OAT delivery performance. Attributes of interest include hospital structural characteristics (e.g., size), social structures (e.g., organizational culture and values), and external attributes (e.g., local and federal policies, and the behavior of other hospitals). Service delivery in the hospital is predominantly dictated by the availability of hospital resources. For example, if a hospital does not have OAT on the formulary, or stocked in the inpatient pharmacy, a hospitalized patient cannot access OAT. The patient's perspective on hospital OAT delivery was not directly addressed and is a study limitation. As proxies for patientinformed data, the study assessed the influence of demand-side attributes using the qualitative and quantitative data. Key informants discussed their perceived barriers for patients (i.e., the demand-side), and the Veterans Health Administration (VHA) data included demographic information (e.g., patient age) and characteristics about the admission (e.g., length of stay).

The three categories of outcomes for this study were hospital OAT delivery (e.g., OAT receipt during hospitalization), clinical outcomes (e.g., in-hospital mortality), and 
post-admission health services utilization (e.g., hospital readmission, emergency

department visits, and OAT receipt). Table 3.1 defines and operationalizes the

overarching research question and the method and data source for each element.

Table 3.1. Primary Research Question Operationalized

\begin{tabular}{|c|c|c|}
\hline Concept & Definition & $\begin{array}{l}\text { Method \& } \\
\text { Data Source }\end{array}$ \\
\hline \multicolumn{3}{|l|}{ Supply-Side Attributes } \\
\hline Inside the Hospital & $\begin{array}{l}\text { Social structures (e.g., hospital standards of } \\
\text { care), technology and resources (e.g., hospital } \\
\text { policies), patient population (e.g., OUD patient } \\
\text { volume), and physical structure (e.g., } \\
\text { geographic location) }\end{array}$ & $\begin{array}{l}\text { Quantitative } \\
\text { Qualitative }\end{array}$ \\
\hline Outside the Hospital & $\begin{array}{l}\text { Social structures (e.g., behavior of other } \\
\text { organizations) and technology and resources } \\
\text { (e.g., treatment policies) }\end{array}$ & Qualitative \\
\hline \multicolumn{3}{|l|}{ Outcomes } \\
\hline Hospital OAT Delivery & $\begin{array}{l}\text { Hospital OAT delivery during an OUD-related } \\
\text { admission, further specified by care delivery } \\
\text { mechanism: OAT continued, OAT } \\
\text { initiation/linkage; OAT sustained; OAT } \\
\text { withdrawal }\end{array}$ & Quantitative \\
\hline Admission & $\begin{array}{l}\text { In-hospital mortality; leaving against medical } \\
\text { advice }\end{array}$ & Quantitative \\
\hline Post-Admission & $\begin{array}{l}\text { Events in the } 30 \text { days after discharge: Death; } \\
\text { readmission; emergency department visit; and } \\
\text { OAT receipt }\end{array}$ & Quantitative \\
\hline
\end{tabular}

\section{Section 3.2. Study Design, Specific Aims, and Research Sub-Questions}

An integrated mixed method sequential explanatory research design with three aims investigated the contributions of multilevel supply-side attributes on hospital OAT delivery. Aim 1 is the qualitative portion of the study and Aims 2 and 3 rely upon the quantitative data (see Figure 3.1). 


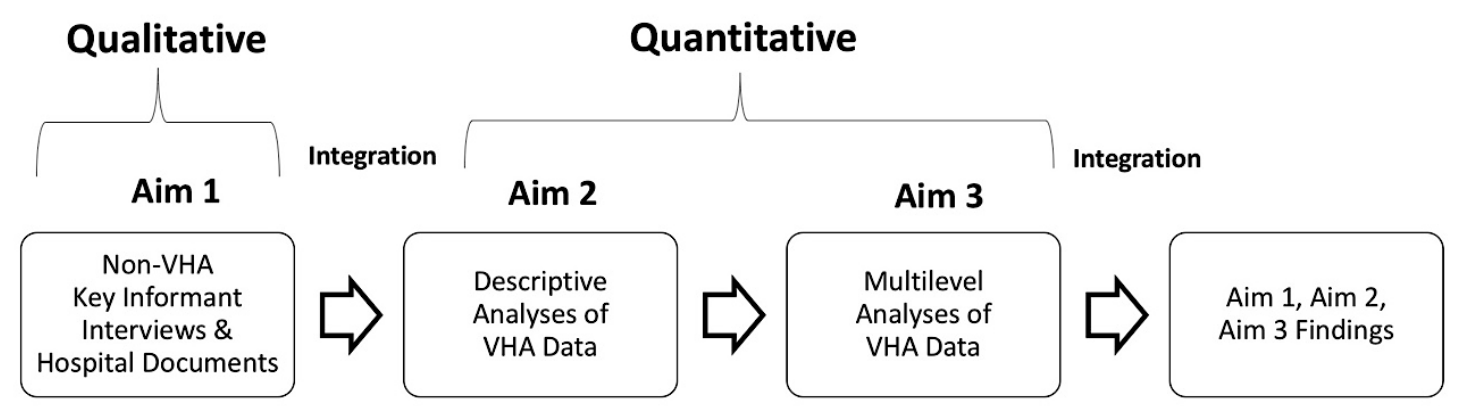

Figure 3.1. Study Design Diagram

A directed content analysis (Aim 1) of 17 key informant interview transcripts

from 16 non-VHA hospitals and a framework analysis of 25 hospital guidance documents from 10 non-VHA hospitals examined how addiction medicine experts and their hospitals delivered OAT for patients with OUD. The study concluded with analyses of two quantitative aims (Aims 2 and 3) using administrative data from a retrospective national sample of patients hospitalized with OUD within the VHA system, fiscal year 2017. The study's three aims and 16 research sub-questions are presented below:

Aim 1: Examine the influence of supply-side attributes inside and outside of non-VHA hospitals that shape hospital OAT delivery.

- Q1a: How is OAT delivered for patients with OUD in the hospital setting?

- Q1b: Which internal and external environmental supply-side elements facilitate hospital OAT delivery?

- Q1c: Which internal and external environmental supply-side elements impede hospital OAT delivery?

- Q1d: How do hospital policies and guidance documents inform hospital OAT delivery? 
Aim 2: Describe and test variation in VHA hospital OAT delivery.

- Q2a: Do the characteristics of the patients who received OAT differ from those who did not receive OAT while hospitalized?

- Q2b: Does non-OAT pharmacotherapy delivery differ for patients who received OAT and for those who did not receive OAT while hospitalized?

- Q2c: Do outcomes differ for patients who received OAT and for those who did not receive OAT while hospitalized?

- Q2d: Do the characteristics of the patients who were discontinued from OAT differ from those who were continued on OAT at admission?

- Q2e: Does non-OAT pharmacotherapy delivery differ for patients who received OAT and for those who did not receive OAT while hospitalized?

- Q2f: Do outcomes differ for patients who received OAT and for those who did not receive OAT while hospitalized?

- Q2g: Are specific OAT care scenarios associated with hospital OAT delivery quartile?

- Q2h: Which hospital attributes are associated with hospital OAT delivery?

- Q2i: Are non-OAT pharmacotherapy services associated with hospital OAT delivery quartile?

- Q2j: Were outcomes associated with hospital OAT delivery performance? 
Aim 3: Test associations among patient and hospital characteristics on VHA hospital OAT delivery.

- Q3a: How much of the variation in OAT delivery is attributable to the hospitals?

- Q3b: How do patient and hospital attributes affect hospital OAT delivery?

The Aim 1 research questions identified supply-side attributes inside and outside the hospital that explain, in part, variability in hospital OAT care and the common practices used to enhance hospital treatment of persons with OUD. The Aim 2 research questions described and explored care practices and variables associated with VHA hospital OAT delivery variation. The Aim 3 research questions tested for the contributions of the multilevel attributes on OAT care variation.

\section{Section 3.3. Research Approach Justification and Study Assumptions}

This section discusses the use of an integrated quantitative and qualitative mixed methods study from paradigmatic and methodologic perspectives (Creswell \& Clark, 2011), and concludes with study assumptions. Over many years the definition of "mixed methods" has varied (Creswell \& Clark, 2011). Mixed methods may refer to a research philosophy, design, or methodologic approach (Creswell \& Clark, 2011). Creswell and Clark (2011) summarized the six core characteristics inclusive of these different viewpoints. In mixed methods, the researcher:

- Mixes, integrates or links the two forms of data either concurrently, sequentially, or through embedding one within the other;

- Prioritizes one or both forms of data; 
- Uses both techniques in a single study;

- Frames research techniques in a paradigm and a theoretical lens; and

- Combines the methods into a specific research design guiding the study (Creswell \& Clark, 2011).

In the 1970s and 1980s, the mixed methods approach was subject to a philosophical debate centered on whether combining quantitative and qualitative approaches was possible because of their different and seemingly conflicting research paradigms. A research paradigm is a shared belief system among a research community that dictates a field's salient questions and dominant use of research techniques (Kuhn, 1970). During this debate, the purists held that mixing quantitative and qualitative paradigms was philosophically and theoretically impossible (Rossman \& Wilson, 1985), the situationalists asserted that adaptation of research paradigms and methods for specific situations was possible (Creswell \& Clark, 2011), and the pragmatists promoted the use of multiple paradigms to address a singular research problem (Rossman \& Wilson, 1985). Thus, a pragmatic research paradigm is inclusive of other research worldviews such as post-positivism or constructivism to meet the needs of the research question (Creswell \& Clark, 2011).

3.3.1. Pragmatic research paradigm. The ontology, epistemology, axiology, and methodology (Creswell \& Clark, 2011; Crotty, 1998) of the pragmatic research paradigm support the mixed methods approach used for this dissertation. A pragmatic research philosophy asserts that the observation of a consequence or outcome furthers the 
understanding of both the behavior and the beliefs that led to that outcome; thus, actions cannot be separated from context, actions are linked to mutable consequences, and actions depend on a socially shared set of beliefs (Morgan, 2014). Ontology is the orientation of reality, and in pragmatism, ontology holds that singular and multiple perspectives exist, so researchers test hypotheses and provide multiple perspectives (Creswell \& Clark, 2011). Epistemology is the relationship between the researcher and the research, and in pragmatism this relationship is one of practicality, meaning that the researcher collects data from a "what works" approach to address the research question (Creswell \& Clark, 2011). Axiology is the role of values in research and in pragmatism this includes biased and unbiased perspectives (Creswell \& Clark, 2011). Finally, the primary methodology of a pragmatic paradigm uses a mix of quantitative and qualitative data collection and analyses (Creswell \& Clark, 2011).

3.3.2. Study assumptions. There were several assumptions reflected in the rationale, the research questions, the design, and analysis of this dissertation. The first assumption was that OUD is a "medical condition" even though it may be better understood as a complex manifestation of interacting contributory processes that exist at the macro and micro-levels (e.g., individual pre-disposition; social, historical, and cultural environment; neurobiology). A second assumption was that hospital-based OAT delivery is a solution to caring for persons with OUD. The third assumption was that demand-side attributes have a minimal contribution to hospital OAT delivery; for example, some patients may refuse hospital OAT for unknown reasons. Fourth, the 
study assumed that OAT is a better treatment approach in the hospital than non-OAT opioids, although a direct comparison has never been studied. A fifth assumption posited that hospital finances, rather than patient and provider needs, drive many hospital decisions.

\section{Section 3.4. Human Subjects Protections}

On January 8, 2018, the OHSU Institutional Review Board (IRB) [IRB \#18092] approved Aim 1 (the qualitative study), and on May 14, 2018, the Veterans Affairs (VA) IRB [IRB \#4045] approved Aims 2 and 3 (the quantitative study). Both IRBs determined each of the study components to be minimal risk human subjects research. The primary risks for Aim 1 were minimized by using an OHSU IRB approved information sheet prior to interview initiation (see Appendix B), by requesting consent to participate and oral consent for recording the interviews, and by labeling the qualitative data with unique identifying codes instead of names. The primary risks for Aim 2 and 3 were minimized by receiving a deidentified dataset (e.g., no dates of service, birthdate, medical record number, social security number, or personal address) and by excluding hospitals with less than 25 admissions from analyses.

\section{Section 3.5. Aim 1: Qualitative Analyses}

The qualitative analyses used two different, albeit similar, analytic approaches: directed content analysis (Hsieh \& Shannon, 2005) for the key informant interview transcripts and a framework analysis (Ritchie \& Spencer, 1994) for the hospital guidance documents. A directed content analysis is a primarily deductive approach effective for validating or extending prior theoretical frameworks (Hsieh \& Shannon, 2005). A 
framework analysis, an approach developed for applied social policy research, has seven fundamental principles: 1) it is grounded or generative; 2 ) it is dynamic; 3 ) it is systematic; 4) it is comprehensive; 5) it is easily retrieved; 6) it allows between- and within-case analysis; and 7) it is accessible to others outside the study team (Ritchie \& Spencer, 2002).

3.5.1. Data sources and study cohort. The Aim 1 study cohort included $17 \mathrm{key}$ informants from 16 U.S. non-VHA hospitals. The cohort members were national hospital OUD experts who were professionally involved in direct care, research, or the development of addiction-related hospital policies, procedures, and practices for patients with SUD. They were addiction medicine physicians who were identified directly or indirectly through the publicly available 2018-2019 fellowship list from the Addiction Medicine Foundation (Addiction Medicine Foundation, 2018). The qualitative data included 17 transcribed 45-to 60-minute key informant interviews, informant demographic information, interview notes, and 25 hospital guidance documents from 10 of the 16 non-VHA hospitals.

3.5.2. Study sample selection and recruitment. Informants were recruited in two waves of email sampling. The IRB approved email recruitment script is in Appendix C. Potential key informants received email invitations to participate a minimum of once and a maximum of three times. The email requested contact information for the most appropriate person to speak with at their institution, which included themselves. 
Recommendations from dissertation mentors and respondent-driven recommendations supplemented recruitment.

A word search of the publicly available Addiction Medicine Foundation's 2018 to 2019 fellowship list (Addiction Medicine Foundation, 2018) for the word "consult" identified 26 fellowships that appeared to include addiction medicine consult services. Twenty-five of the 26 programs were contacted with an invitation to participate in the study; one program was excluded because the program director's email address was not provided. Of the 25 programs contacted 13 individuals from 12 fellowship programs completed interviews.

After exhausting the first recruitment sample, a second wave of sampling proceeded with the remaining 20 programs that did not have the word "consult" in the program description, excluding the two Canadian programs and three primarily based in the VHA. The Canadian and VHA programs were excluded to focus the sampling frame and increase the homogeneity of the types of hospitals and surrounding environments within the study sample. From the remaining 20 programs, 11 program directors were contacted based on geographic location, expert recommendation, respondent recommendation, and publicly available programmatic information. Of the 11 additional programs contacted, four additional individuals from four different institutions participated in the study. 
3.5.4. Data collection and study tools. The OHSU IRB approved study tools included: 1) the key informant demographic survey (Appendix D); and 2) the semistructured interview guide (Appendix E).

3.5.4a. Key informant demographic survey. Key informants completed a demographic survey at the start of the interview providing basic personal and professional demographic information and OAT-related characteristics at the hospital of employment. Question examples included: "What health professional degree(s) do you have?" and "For how many years have you worked at your respective hospital?"

3.5.4b. Semi-structured interview guide. The conceptual framework and the guidance of addiction medicine experts shaped the development of the semi-structured interview guide. The final interview guide probed four topics to identify external and hospital supply-side attributes related to hospital OAT service delivery: 1) development and implementation of known effective care delivery mechanisms for OUD treatment in the hospital setting, focused on addiction medicine consult (AMC) services; 2 ) OUD care delivery policies, procedures, and practices; 3) hospital barriers and facilitators to hospital OUD treatment and OAT delivery; and 4) environmental barriers and facilitators to hospital OUD treatment and OAT delivery.

Informants completed 45- to 60-minute telephone interviews with the primary researcher. Participants did not receive a copy of the interview guide. Interviews were electronically recorded. When a specific hospital policy was mentioned during the 
interview, a copy was requested via email after the interview to obtain electronic copies of the documents. Interviews were transcribed by the primary researcher.

3.5.5. Data analysis. The data sources analyzed included: informant demographic survey data; key informant interview transcripts; and hospital policies.

3.5.5a. Key informant demographic surveys. Key informant demographic surveys provided a professional and educational context for the perspectives of the informants and their current care delivery environment. Descriptive summaries, such as mean age of the key informants, were generated using Microsoft Excel (Microsoft, 2018) to contextualize the study findings.

3.5.5b. Key informant interview transcripts. Informant interviews were coded using a directed content analysis approach (Hsieh \& Shannon, 2005). Dedoose (SocioCultural Research Consultants LLC, 2016), a qualitative analysis software, was used to organize and manage the qualitative data and analytic process. The conceptual framework served informed the creation of the preliminary codebook prior to data collection.

Analysis of the interviews began prior to the completion of all interviews. Each interview was coded and re-coded in iterative cycles. Initially the interviews were coded with the draft codebook (Hsieh \& Shannon, 2005). Throughout the course of data collection and analysis, the codebook was modified to reflect emergent findings (Hsieh \& Shannon, 2005). Important and relevant data that did not fit clearly into the original 
coding scheme inspired the creation of new categories and codes (Hsieh \& Shannon, 2005).

The original codebook had five categories, with 23 codes: 1) environmental attributes; 2) hospital supply-side attributes; 3) demand-side attributes; 4) AMC service attributes; and 5) current hospital policies. The final codebook was expanded to eight categories, with 59 codes: 1) environmental attributes; 2) hospital supply-side attributes; 3) demand-side attributes; 4) AMC service attributes; 5) hospital policy and practice; 6) financing; 7) other models of care; and 8) care delivery topics. Changes to the codebook reflected the focus and the granularity of information provided during the interviews. Financing was added, as a single higher-level category, because it was a frequently described and observed to be intrinsically connected to the other categories. The category "hospital policies and practice" was expanded to include not only the discussion of documented hospital policies but also typical practices because several informants disclosed that their standard of care or common practices were not formally documented. See Appendix F for a copy of the final codebook and frequency counts of categories and codes.

Upon the completion of primary coding, a second coder (the dissertation committee chair) reviewed the categories and codebook for all 17 coded interviews and provided additional coding suggestions. The primary researcher reconciled the coding changes and any discrepancies in codes were discussed as a research team until consensus was reached. All documents received a second coding review by the primary 
coder to identify any text that was missing a relevant code or when redundant coding occurred. The final themes presented in Chapter 4 were created through the consolidation of the eight categories from the codebook, organized around the study's conceptual framework.

3.5.5c. Hospital guidance documents. A framework analysis approach guided the analyses of the hospital guidance documents (Ritchie \& Spencer, 2002). This approach begins with becoming familiar with the documents for analysis, identifying a thematic framework, indexing the policies, charting the policies, and completing the analysis and policy interpretation (Ritchie \& Spencer, 2002). The preliminary thematic framework for this study was informed by expert opinion and a priori policy categories: 1) OAT continuation; 2) withdrawal management; 3) OAT induction; and 4) pain management. The framework was modified throughout the course of data collection and data familiarization with the 25 policies. The thematic framework expanded to include five policy categories: 1) OAT continuation; 2) opioid withdrawal management; 3) OAT induction; 4) pain management; and 5) security and behavioral management policy.

During the indexing and charting processes, policies were collapsed into two broad policy themes comprised of eight policy sub-categories: 1) OAT management and 2) security and behavioral management. Qualitative tables were created to summarize and organize findings by the broader themes and sub-categories. Thirty-three pages of 
text summarized the policies and supported the policy indexing and charting processes. This documentation may be requested from the primary researcher.

3.5.6. Linking Aim 1 to Aims 2 and 3. The qualitative findings presented in Chapter 4 provided the rationale for continuing to explore care delivery practice, patterns, and organizational processes for hospitalized patients with OUD. The qualitative findings also informed the subsequent coding of hospital OAT in the VHA quantitative dataset, and the analyses of Aims 2 and 3 (reported in Chapter 5).

\section{Section 3.6. Aims 2 and 3: Quantitative Analyses}

The exploration of the Aims 2 and 3 research questions occurred through the use of pre-specified statistical analytic techniques. The rationale for the use of these techniques was based on the evidence presented in the Chapter 2 literature review including previously conducted research on hospital care variation (Safavi et al., 2014). The Aim 2 analytic techniques included descriptive statistics (e.g., frequency, range, median, interquartile range [IQR]) and bivariate statistical tests to assess for differences among groups of patients and hospital OAT delivery quartiles (e.g., Pearson chi-square and Mann-Whitney $U$ tests). The Aim 3 analytic technique used multilevel and multivariable logistic regression models, justified both theoretically and statistically (Luke, 2004). Two data analysis programs were used: 1) RStudio (RStudio Team, 2015) for data management, coding, and for bivariate statistical tests; and 2) Stata (StataCorp, 2017) for the multilevel logistic regression models. The RStudio packages are provided in 
Appendix G (Dinno, 2017; Fox \& Weisberg, 2011; Revelle, 2018; Wasey, 2018; Wickham, 2011, 2017).

3.6.1. Data sources and study cohort. A retrospective sample of VHA patients was obtained from the VHA Corporate Data Warehouse. The VHA Corporate Data Warehouse is a collection of databases accessed securely through the VHA Informatics and Computing Infrastructure Workspace. This data source provides real-time demographic, medical, and pharmacy health services utilization data for veterans who receive care in the VHA system. In these databases patient data are linked across multiple tables (e.g., diagnoses, outpatient visits, inpatient admissions, outpatient prescription, methadone clinics) through a unique numeric identifier.

Each case was a unique patient admission in the VHA system. Patients were included in this study if they had an OUD diagnosis (see Appendix H) from any source, inpatient or outpatient, between October 1, 2015 and October 1, 2017, in the year preceding the admission date of the index hospitalization as defined below. This included clinical scenarios in which an OUD diagnosis occurred during the index hospitalization. Eligible OUD diagnoses could be primary diagnoses coded when OUD was the main reason for the clinical encounter, or as a secondary diagnosis. The admitting facilities were restricted to "acute care hospitals" which was defined as facilities that had at least 500 acute bed days of care during the study year.

Study validity was enhanced through the refinement of the study cohort. Each step to generate the final study cohort was documented in the study consort diagram in 
Appendix I which included the removal of: 1) hospitals and admissions outside the continental U.S. (hospitals $=1$; cases $=134) ; 2$ ) facilities with bed category discrepancies (hospitals $=6$; cases $=141) ; 3$ ) admissions with non-hospital places of admission (cases $=$ 7) or missing the place of admission (cases $=2$ ); 4) admissions missing a primary or secondary admission code (cases $=271) ; 5$ ) admissions with an inconsistent death code (hospitals $=1$, cases $=4$ ); 6 ) admissions with a cancer flag (cases $=2,062$ ), as defined by the Elixhauser flags (AHRQ, 2018), including cases with a solid tumor without metastasis, metastatic cancer, or lymphoma present on admission; 7) admissions with a length of stay in the $99^{\text {th }}$ percentile (hospitals $=1$; cases $=130$ ); and 8 ) hospitals with less than 25 admissions (hospitals $=21$; cases $=262$ ). The final dataset included 12,407 admissions from 109 unique VHA hospitals within the continental U.S. There were no facilities located in New Hampshire or Nebraska. Appendix J has additional details on the study cohort definitions.

3.6.2. Quantitative variables. Variable selection was informed by the existing literature, the data available from the VHA data source, and results of the qualitative portion of the study. There were three time points of interest in this study: 1) 30 days prior to index hospitalization ("pre-admission"); 2) index hospitalization ("admission"); and 3) 30 days post index hospitalization ("post-admission").

3.6.2a. Index hospitalization definition. The definition of an index hospital admission was the first hospital admission during the 2017 VHA fiscal year (October 1 2016 to October 1, 2017) for veterans (aged 18 and older) with an OUD-related ICD-10 
diagnosis code (Appendix $\mathrm{H}$ ) as an inpatient or outpatient, or within the FEE BASIS care setting within the previous 12 months from the index hospital admission date, which could include a diagnosis during index admission. FEE BASIS care refers to non-VHA clinical care paid for by VHA on a veteran's behalf, typically due to the service not being offered by VHA or because the geographic distance from the patient to the VHA service presents a barrier to access services.

3.6.2b. Patient and admission variables. The patient and admission variables were either present upon data receipt or constructed and coded from the data; see Table 3.2 for summarized definitions. Appendix $\mathrm{K}$ includes additional details on variable construction and Appendix $L$ is the list of ICD-10 codes queried for co-occurring mental health conditions and co-occurring SUDs. 
Table 3.2. Patient and Admission Variables

\begin{tabular}{|c|c|c|}
\hline Variable & Type & Definition \\
\hline \multicolumn{3}{|c|}{ Patient Characteristics } \\
\hline Age & Continuous & Age in years present on admission \\
\hline $\begin{array}{l}\text { Co-Occurring } \\
\text { Mental Health }\end{array}$ & $\begin{array}{l}\text { Binary/ } \\
\text { Categorical }\end{array}$ & $\begin{array}{l}\text { 1) Adjustment Disorder Other; 2) Anxiety Disorder; 3) Mood Disorder; } \\
\text { 4) Non-Mood Psychotic disorder; 5) PTSD; 6) Self-Harm; codes in } \\
\text { Appendix L }\end{array}$ \\
\hline $\begin{array}{l}\text { Co-Occurring } \\
\text { SUD }^{1}\end{array}$ & $\begin{array}{l}\text { Binary/ } \\
\text { Categorical }\end{array}$ & $\begin{array}{l}\text { 1) Alcohol Use Disorder; 2) Cannabis Use Disorder; 3) Cocaine Use } \\
\text { Disorder; 4) Hallucinogen Use Disorder; 5) Nicotine Dependence; 6) } \\
\text { Other Psychoactive Use Disorders; 7) Other Stimulant Related } \\
\text { Disorders; 8) Other Substance Use Disorder; 9) Sedative Hypnotic } \\
\text { Disorders; codes in Appendix L }\end{array}$ \\
\hline Gender & Binary & Male or Female \\
\hline Ethnicity & Categorical & 1) Not Hispanic or Latino; 2) Hispanic or Latino; 3) Unknown \\
\hline $\begin{array}{l}\text { OUD-Related } \\
\text { Diagnosis }\end{array}$ & $\begin{array}{l}\text { Binary/ } \\
\text { Categorical }\end{array}$ & $\begin{array}{l}\text { Primary or secondary ICD-10 OUD admission diagnosis codes; codes in } \\
\text { Appendix } \mathrm{H}\end{array}$ \\
\hline $\begin{array}{l}\text { OUD-Related } \\
\text { Infections }^{2}\end{array}$ & $\begin{array}{l}\text { Binary/ } \\
\text { Categorical }\end{array}$ & $\begin{array}{l}\text { 1) Endocarditis; 2) Candida Endocarditis; 3) Osteomyelitis; 4) } \\
\text { Bacteremia; 5) Discitis; 6) Septic Arthritis; 7) Brain Abscess; 8) Joint } \\
\text { Infection; 9) Necrotizing Fasciitis; 10) Empyema; and 11) Lung Abscess, } \\
\text { codes in Appendix H }\end{array}$ \\
\hline $\begin{array}{l}\text { OUD-Related } \\
\text { Diagnosis or } \\
\text { Infection }\end{array}$ & Binary & $\begin{array}{l}\text { Combined variable: OUD-Related Diagnosis or OUD-Related Infection } \\
\text { Diagnosis }\end{array}$ \\
\hline $\begin{array}{l}\text { Primary } \\
\text { Diagnosis }\end{array}$ & Categorical & The primary ICD-10 admission diagnosis code for index hospitalization \\
\hline Race & Categorical & $\begin{array}{l}\text { 1) American Indian or Alaska Native; 2) Asian; 3) Black or African } \\
\text { American; 4) White; 5) Native Hawaiian or Other Pacific Islander; 6) } \\
\text { Unknown/Decline to Answer }\end{array}$ \\
\hline $\begin{array}{l}\text { Secondary } \\
\text { Diagnosis }\end{array}$ & Categorical & $\begin{array}{l}\text { The secondary ICD-10 admission diagnosis code for index } \\
\text { hospitalization }\end{array}$ \\
\hline \multicolumn{3}{|c|}{ Admission Characteristics } \\
\hline $\begin{array}{l}\text { Admission } \\
\text { Source }\end{array}$ & Categorical & $\begin{array}{l}\text { Point of admission for the index hospitalization: 1) Outpatient } \\
\text { Treatment; 2) Other Direct admission; 3) Other }\end{array}$ \\
\hline ICU Service & Binary & Use of ICU services during index hospitalization \\
\hline Length of Stay & Continuous & Length of time (days) of the index hospitalization \\
\hline Surgical Service & Binary & Use of surgical services during index hospitalization \\
\hline
\end{tabular}

3.6.2c. Non-OAT pharmacotherapy variables. Pharmacotherapy variables were

coded for the three study time periods: 1) pre-admission, which included filled

prescriptions or medication procedure codes; 2) admission, which included

administered medication, filled prescriptions ( $<1 \%$ of data points), or medication

procedure codes; and 3) post-admission, which included filled prescriptions or 
medication procedure codes. If a medication was used more than once in a study time period, it was only counted once. See Table 3.3 for definitions and Appendix $\mathrm{N}$ for additional details.

Table 3.3. Non-OAT Pharmacotherapy Variables ${ }^{1}$

\begin{tabular}{|c|c|}
\hline Variable & Definition \\
\hline \multicolumn{2}{|l|}{ Pre-Admission } \\
\hline Benzodiazepine & Prescription filled for any Benzodiazepine \\
\hline Concurrent Opioid/Benzodiazepine & Prescription filled for any Opioid and any Benzodiazepine \\
\hline Gabapentin/Pregabalin & Prescription filled for Gabapentin or Pregabalin \\
\hline Naloxone & Prescription filled for Naloxone \\
\hline Naltrexone & Prescription filled or HCPCS code for Naltrexone \\
\hline Opioid & $\begin{array}{l}\text { Prescription filled for any Non-OAT Opioids, not including } \\
\text { Methadone or Buprenorphine }\end{array}$ \\
\hline \multicolumn{2}{|l|}{ Admission $^{2}$} \\
\hline Benzodiazepine & Administration of Benzodiazepine \\
\hline Gabapentin/Pregabalin & Administration of Gabapentin or Pregabalin \\
\hline Naltrexone & Administration of Naltrexone \\
\hline No Opioid and No OAT & No administration of an Opioid and OAT \\
\hline Opioid & $\begin{array}{l}\text { Administration of any Non-OAT Opioid, did not include } \\
\text { Methadone or Buprenorphine formulations for pain }\end{array}$ \\
\hline First-Line Withdrawal Adjuvant & Administration of Clonidine \\
\hline Second-Line Withdrawal Adjuvant ${ }^{3}$ & $\begin{array}{l}\text { Administration of any second-line adjuvant: Baclofen or } \\
\text { Gabapentin or Pregabalin or Tizanidine }\end{array}$ \\
\hline Any Withdrawal Adjuvants & $\begin{array}{l}\text { Administration of any of the adjuvants: Baclofen or } \\
\text { Clonidine or Gabapentin or Pregabalin or Tizanidine }\end{array}$ \\
\hline \multicolumn{2}{|l|}{ Post-Admission } \\
\hline Benzodiazepine & Prescription filled for any Benzodiazepine \\
\hline Concurrent Opioid/Benzodiazepine & Prescription filled for any Opioid and any Benzodiazepine \\
\hline Gabapentin/Pregabalin & Prescription filled for Gabapentin or Pregabalin \\
\hline Naloxone & Prescription filled for Naloxone \\
\hline Naltrexone & Prescription filled or HCPCS code for Naltrexone \\
\hline \multicolumn{2}{|c|}{$\begin{array}{l}\text { Table Notes. }{ }^{1} \text { Appendix } N \text { describes variable construction; }{ }^{2} \text { during admission }<1 \% \text { of pharmaceutical } \\
\text { data points were prescribed; }{ }^{3} \text { Second line adjuvants are medications recommended for use by VHA } \\
\text { Opioid Taper Tool (U.S. Department of Veterans Affairs, 2016b); HCPCS = Healthcare Common } \\
\text { Procedure Coding System is a specific procedure billing code for medication administration. }\end{array}$} \\
\hline
\end{tabular}

3.6.2d. OAT pharmacotherapy variables. OAT pharmacotherapy variables were created for the three study time periods. The pre-and post-admission OAT variables included filled buprenorphine prescriptions, procedure codes for methadone administration and non-specific OAT administration, or OTP visit documentation. The 
any OAT admission variable included all formulations of buprenorphine and methadone and any OAT-related procedure codes. The decision to include non-FDA approved OAT formulations (e.g., injectable methadone) was based on the findings from Aim 1 and the federal rules that allow this practice. Table 3.4 provides a summary of the OAT variable definitions and Appendix O provides additional details.

Table 3.4. OAT Variables ${ }^{1}$

\begin{tabular}{|c|c|c|}
\hline Variable & Definition & Pharmacotherapy \\
\hline \multicolumn{3}{|l|}{ Pre-Admission } \\
\hline Any OAT & $\begin{array}{l}\text { Binary/ } \\
\text { Categorical }\end{array}$ & $\begin{array}{l}\text { Prescription filled for FDA-approved OAT or OAT } \\
\text { Procedure Code or OTP Visit }\end{array}$ \\
\hline \multicolumn{3}{|l|}{ Admission } \\
\hline Any OAT & $\begin{array}{l}\text { Binary/ } \\
\text { Categorical }\end{array}$ & Administration of Any OAT \\
\hline OAT Continuation & Binary & $\begin{array}{l}\text { OAT delivered during pre-admission and } \\
\text { admission, but not post-admission }\end{array}$ \\
\hline OAT Initiation \& Linkage to Care & Binary & $\begin{array}{l}\text { OAT delivered during admission and post- } \\
\text { admission, but not pre-admission }\end{array}$ \\
\hline OAT Sustained & Binary & $\begin{array}{l}\text { OAT delivered pre-admission, during admission } \\
\text { and post-admission }\end{array}$ \\
\hline OAT Withdrawal & Binary & $\begin{array}{l}\text { OAT delivered during admission, but not pre or } \\
\text { post-admission }\end{array}$ \\
\hline OAT with Adjuvant & Binary & $\begin{array}{l}\text { Administration of OAT and } \\
\text { an any adjuvant during admission }\end{array}$ \\
\hline OAT with First-line Adjuvant & Binary & $\begin{array}{l}\text { Administration of OAT and } \\
\text { a first-line adjuvant during admission }\end{array}$ \\
\hline OAT with Second-line Adjuvant & Binary & $\begin{array}{l}\text { Administration of OAT and } \\
\text { a second-line adjuvant during admission }\end{array}$ \\
\hline OAT with Opioid & Binary & $\begin{array}{l}\text { Administration of OAT and } \\
\text { an any Non-OAT Opioid during admission }\end{array}$ \\
\hline OAT with Naltrexone & Binary & $\begin{array}{l}\text { Administration of OAT and } \\
\text { naltrexone during admission }\end{array}$ \\
\hline \multicolumn{3}{|l|}{ Post-Admission } \\
\hline Any OAT & Binary & $\begin{array}{l}\text { Prescription filled for FDA-approved OAT } \\
\text { or OAT Procedure Code or OTP Visit }\end{array}$ \\
\hline
\end{tabular}


3.6.2e. Patient health and utilization variables. Patient health and services

utilization variables were queried either directly from the VHA data or constructed from the data through coding. Definitions are provided in Table 3.5 and additional details are in Appendix $\mathrm{P}$.

Table 3.5. VHA Patient Health and Utilization Variables ${ }^{1}$

\begin{tabular}{|c|c|c|}
\hline Variable & Type & Definition \\
\hline In-Hospital Mortality & Binary/Categorical & Death during admission \\
\hline Left Against Medical Advice & Binary/Categorical & $\begin{array}{l}\text { Leaving against medical advice during } \\
\text { admission }\end{array}$ \\
\hline Death & Binary & $\begin{array}{l}\text { Death within the post-admission time } \\
\text { period }\end{array}$ \\
\hline Emergency Department Visit & Binary & $\begin{array}{l}\text { VHA emergency department visit within } \\
\text { the post-admission time period }\end{array}$ \\
\hline Hospital Readmission & Binary & $\begin{array}{l}\text { VHA acute care visit within the post- } \\
\text { admission time period }\end{array}$ \\
\hline
\end{tabular}

3.6.2f. Hospital characteristic variables. Hospital characteristic variables were queried either directly from the VHA data or constructed through coding. Definitions are provided in Table 3.6 and additional details are in Appendix Q.

Table 3.6. VHA Hospital Characteristic Variables ${ }^{1}$

\begin{tabular}{|c|c|c|}
\hline Variable & Type & Definition \\
\hline Acute OUD Diagnosis Volume & Binary & $\begin{array}{l}\text { The proportion of index admissions in a } \\
\text { facility with an acute OUD diagnosis (OUD- } \\
\text { infection or OUD diagnosis) }\end{array}$ \\
\hline Admission Volume & Continuous & The number of admissions in a facility \\
\hline Hospital Region & Categorical & $\begin{array}{l}\text { U.S. Census categories: 1) Northeast; 2) } \\
\text { Midwest; 3) South; 4) West }\end{array}$ \\
\hline Hospital Size & Categorical & $\begin{array}{l}\text { 1) Small: } 1 \text { to } 49 \text { beds; 2) Medium: } 50 \text { to } 99 \\
\text { beds; 3) Large: } 100+\end{array}$ \\
\hline
\end{tabular}

\subsubsection{Descriptive and bivariate analyses. Frequencies and percentages were} calculated for hospital (admission volume, size, and location), patient (age, gender, race, and ethnicity), and admission (length of stay, admission source, and service acuity) 
characteristics across the study cohort population $(n=12,407)$. The frequency of patient health-related characteristics was also calculated for OUD-related infection diagnosis, acute OUD-related diagnosis, the top 10 most common primary ICD-10 diagnosis codes, top 10 most common secondary ICD-10 diagnosis codes, co-occurring mental health conditions, and co-occurring SUDs. Pharmacotherapy frequency for the entire study cohort was described for non-OAT and OAT in all three study time periods. Additional system-wide descriptions of hospital OAT delivery across the study cohort and all facilities were conducted for any OAT delivery (frequency, median, range, IQR, skew, and kurtosis) and the four OAT scenarios: 1) OAT continued; 2) OAT initiation and linkage to care; 3) OAT sustained; and 4) OAT withdrawal management.

Bivariate analyses compared sub-groups within the study cohort using Pearson chi-square, Pearson chi-square with Yates' continuity correction, and Mann-Whitney U tests. Sub-group comparisons were made between patients who received OAT and those who did not receive OAT during admission, and patients who were on OAT prior to admission and discontinued with those who were continued on OAT during admission. Differences were assessed for characteristics present on admission, admission characteristics, non-OAT pharmacotherapy delivery during admission, and health and utilization outcomes.

3.6.4. Hospital performance analyses. Hospitals were assigned to one of four hospital OAT delivery performance categories dependent on the proportion of admitted patients with OUD in each hospital who received OAT during their admission. Quartile 1 
hospitals had the lowest relative proportion of OAT delivered and Quartile 4 had the highest. Quartiles were described using measures for any OAT delivered including frequency, median, range, IQR, skew, and kurtosis. Differences across and between quartiles were assessed for OAT and non-OAT pharmacotherapy delivery using the Kruskal-Wallis and Dunn's tests. Quartiles were assessed for differences in characteristics and health and utilization outcomes using Dunn's, Kruskal-Wallis, and Fisher's Exact tests.

3.6.5. Multilevel analyses. Using Stata statistical analysis software (StataCorp, 2017), multivariable logistic regression models were constructed to study the association (alpha value 0.05 ) of multilevel attributes on patient OAT receipt during hospitalization.

3.6.5a. Dependent and independent variables. The dependent variable (level 1) was OAT receipt (yes/no) during hospitalization. The independent variables were continuous, binary, or categorical variables categorized as level 1 (i.e. patient) or level 2 (i.e., hospital). Independent variable inclusion was based on the literature review, expert opinion, prior study aims, and model fit. Level 1 and level 2 independent variables are summarized in Table 3.7. 
Table 3.7. Independent Variables

\begin{tabular}{|c|c|}
\hline Level 1: Patient and Admission Characteristics & Level 2: Hospital Characteristics \\
\hline 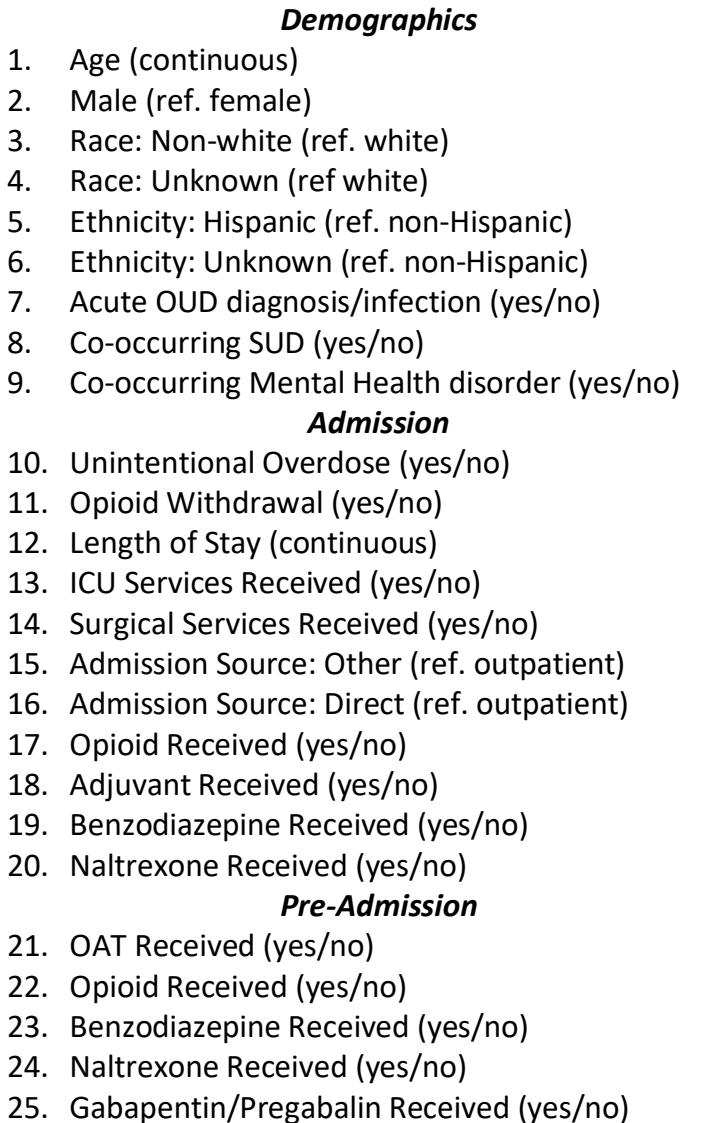 & $\begin{array}{l}\text { 1. Admission Volume } \\
\text { 2. Acute OUD Diagnoses Relative Volume } \\
\text { 3. Hospital Size-Medium (ref. small) } \\
\text { 4. Hospital Size-Large (ref. small) } \\
\text { 5. Census Region Midwest (ref. South) } \\
\text { 6. Census Region Northeast (ref. South) } \\
\text { 7. Census Region West (ref. South) }\end{array}$ \\
\hline
\end{tabular}

3.6.5b. Model preparation. Model preparation began with assessments for multicollinearity among covariates using variance inflation factors (VIFs), the conditional index, and Pearson correlation. Covariates included in the models were below collinearity thresholds - VIF ( $<10)$, conditional index $(<30)$ (Hair, Tatham, Anderson, \& Black, 1998), variance-decomposition matrix (<0.9) (Hair et al., 1998), Pearson's correlation coefficient (<0.8) (Midi, Sarkar, \& Rana, 2010)-except for one covariate with an elevated conditional index (> 30; = 33.5).

3.6.5c. Model building. Model building occurred in a stepwise approach from simple (Model 1) to more complex (Model 2 and 3). The model equations are in Figure 
3.2 and the purpose of each of model and the equation elements are described below.

logit (odds level 1 outcome $)_{i j}=\gamma_{00}+u_{0 j}$

logit (odds level 1 outcome $)_{i j}=\gamma_{00}+\gamma_{10}(\text { level } 1 \text { covariate })_{i j}+U_{0 j}$

logit (odds level 1 outcome $)_{i j}=\gamma_{00}+\gamma_{10}(\text { level } 1 \text { covariate })_{i j}+\gamma_{01}(\text { level } 2 \text { covariate })_{j}+U_{0 j}$

\section{Figure 3.2. Multilevel Modeling Equations}

The purpose of Model 1, the variance component model, was to describe hospital-specific random effects (Austin \& Merlo, 2017) for patient OAT receipt:

- $\quad i=$ the individual admission;

- $\quad j=$ the specific hospital in which the admission occurred;

- logit (odds level 1 outcome) $)_{i j}=$ the dependent variable (patient OAT receipt);

- $\gamma_{00}=$ the level 2 intercept; and

- $u_{0 j}=$ the variance between hospitals (i.e., the random intercept component).

The purpose of Model 2, the intermediate model, was to test for associations between patient characteristics and patient OAT receipt, and to describe the hospitalspecific random effects (Austin \& Merlo, 2017):

- $\gamma_{10}$ is the effect of the level 1 covariate on the group specific intercept.

The purpose of Model 3, the final model, was to test for associations between patient and hospital characteristics on patient OAT receipt and to describe the hospitalspecific random effects (Austin \& Merlo, 2017):

- $\gamma_{10}$ is the effect of the individual level covariate on the group specific intercept; and 
- $\gamma_{01}$ is the effect of the group level covariate on the group specific intercept.

Comparative model fit testing, for the nested models (Model 1 vs. Model 2 vs.

Model 3), used log-likelihood ratio test, the Akaike Information Criteria (AIC), and the Bayesian Information Criteria (BIC). Regression coefficients, standard errors, odds ratios with $95 \%$ confidence intervals, and the intraclass correlation coefficient $(\text { ICC })^{2}$ were also calculated and reported for all three models.

3.6.5d. Sensitivity analysis. A sensitivity analysis was conducted to discern whether using a narrower OAT administration definition (i.e., the exclusion of injectable forms of methadone/buprenorphine) would change study findings. The results of this analysis are presented in Chapter 5 (Section 5.6.4c and Appendix R).

\section{Section 3.7. Conclusion}

A mixed methods study, based on a pragmatic research paradigm, informed the design, approach, and analyses crafted to address the three study aims of this dissertation. The next three chapters present the study findings: Chapter 4 the qualitative findings, Chapter 5 the quantitative findings, and Chapter 6 the synthesis and integration of the qualitative and quantitative findings.

\footnotetext{
${ }^{2} \mathrm{ICC}=\frac{\tau_{0}^{2}}{\tau_{0}^{2}+\frac{\pi^{2}}{3}}$
} 


\section{Chapter 4: Presentation and Analysis of Qualitative Data}

Parts of this chapter were published in a peer-reviewed journal: Priest, K.C., \& McCarty, D. (2018). Role of the hospital in the 21st Century opioid overdose epidemic: The addiction medicine consult service. Journal of Addiction Medicine, Advance online publication. doi: 10.1097/ADM.0000000000000496.

The purpose of Aim 1 was to identify environmental and hospital supply-side attributes that may explain, in part, the variation in care and limited use of OAT for hospitalized patients with OUD. Four research sub-questions guided this portion of the study:

- Q1a: How is OAT delivered for patients with OUD in the hospital setting?

- Q1b: Which internal and external environmental supply-side elements facilitate hospital OAT delivery?

- Q1c: Which internal and external environmental supply-side elements impede hospital OAT delivery?

- Q1d: How do hospital policies and guidance documents inform hospital OAT delivery?

The conceptual framework, described in Chapter 2, informed Chapter 4 analyses and the presentation of findings. Of particular note, the perspective that hospitals and the activities within them exist is because of dynamic and complex interactions among continuously evolving economic, cultural, political, and socially normative structures and processes (see Table 2.2). The chapter is organized in eight sections: 
1. Section 4.1 describes the study cohort -17 key informants from 16 non-VHA hospitals - to provide the professional and environmental context for study findings.

2. Section 4.2. examines the shared and different organizational characteristics of the nine established hospital AMC services.

3. Section 4.3 explores the supply-side attributes (inside and outside the hospital) that facilitate the development and implementation of AMC services for the nine hospitals with established services and the five hospitals with plans to implement AMC services.

4. Section 4.4 describes the barriers (inside and outside the hospital) for the development and implementation of AMC services and hospital OAT delivery for all 16 hospitals.

5. Section 4.5 briefly explores the demand-side attributes (i.e., patient attributes) that emerged during key informant interviews, although the focus of this study was primarily on the supply-side contributions to care.

6. Section 4.6 outlines six specific emergent supply-side actions beyond the AMC service to improve and enhance OUD-related services in the hospital context.

7. Section 4.7 summarizes hospital guidance document analyses $(n=25)$, which describe the current OAT practices in non-VHA hospitals.

8. Section 4.8 links the qualitative findings to the two quantitative aims. 


\section{Section 4.1. Study Cohort}

4.1.1. Participant demographics. Interviews were completed with 17 physicians affiliated with 16 U.S. hospitals or health systems. Participants were found through the publicly available Addiction Medicine Fellowship program list (Addiction Medicine Foundation, 2018). Key informants were board-certified $(n=16)$ or board-eligible $(n=1)$ addiction medicine physicians (medical or osteopathic doctorate) from five subspecialties-family medicine, internal medicine, obstetrics and gynecology, pediatrics, and psychiatry. The key informant mean age was 47 years. The 9 women and 8 men were predominantly white $(n=16)$ and Non-Hispanic or Latino $(n=15)$, and most had worked or trained in their hospital of employment for more than five years $(n=13)$.

4.1.2. Hospital characteristics. Hospitals were located in the West $(n=4)$, Midwest $(n=4)$, Northeast $(n=5)$, and South $(n=3)$, and most were located in states with Affordable Care Act Medicaid expansion $(n=12)$. OAT products were on all hospital formularies for the treatment of OUD except for one, with some restrictions. Most hospitals $(n=10)$ had affiliated or onsite addiction related services, such as opioid treatment programs (OTPs) and "detox" beds. Three hospitals had OTPs and dedicated "detox" beds, three hospitals had only OTPs, four hospitals had only dedicated detox beds, and six hospitals had neither. Interestingly, the two hospitals without an AMC service or plans to start one either had an affiliated OTP or "detox" beds, potentially indicating that the types of addiction-related resources (AMC service vs. OTP vs. "detox" beds) serve different purposes or facilitate different care production mechanisms. The 
role of the AMC service, as both an input and output of service delivery, was an emergent and important finding that is articulated throughout this chapter.

AMC services functioned as a key care delivery mechanism for persons with OUD. The presentation of results, therefore, begins with an assessment of the status of AMC services (established, planning to start, or not established/no plans to start). Over half of the hospitals, 9 of 16, had established AMC services, five hospitals planned to start an AMC, and two hospitals had no AMC service and no plans to start one. See Table 4.1 for a summary of hospital characteristics by region and available services.

Table 4.1. Hospital Characteristics

\begin{tabular}{l|cccc|cccc}
\hline \multicolumn{1}{c|}{ Hospital Type } & \multicolumn{4}{|c|}{ Region } & \multicolumn{3}{c}{ Affiliated/Onsite Services } \\
& MW & NE & S & W & OTP & Detox & Both & Neither \\
\hline $\begin{array}{l}\text { Established AMC } \\
\text { Service }\end{array}$ & 3 & 3 & 1 & 2 & 1 & 2 & 2 & 4 \\
\hline $\begin{array}{l}\text { Starting AMC } \\
\text { Service }\end{array}$ & 0 & 1 & 2 & 2 & 1 & 1 & 1 & 2 \\
\hline No AMC Service & 1 & 1 & 0 & 0 & 1 & 1 & 0 & 0 \\
\hline Total & & & & & & & \\
\hline
\end{tabular}

Table Notes. $M W=$ Midwest; NE = Northeast $S=$ South; $W=$ West OTP = opioid treatment program; Detox = dedicated hospital beds for detoxification from substances; Both = OTP and dedicated hospital beds for detoxification from substances.

The following sections integrate and synthesize the evidence provided by key informants in the interviews and from the hospital guidance documents. The culmination of these findings provides a first glimpse into an otherwise previously limitedly described care delivery scenario.

\section{Section 4.2. AMC Service Design and Operations}

This section describes shared practices and differences in AMC service design and operations to address research sub-question 1a: How is OAT delivered for patients with OUD in the hospital setting? This was assessed through the lens of the study 
conceptual framework, which focused on technology and resources (e.g., staffing, financing) and social structures. AMC service shared practices and differences emerged across four domains: 1) availability and coverage; 2) home department and team composition; 3) responsibility and scope; and 4) financing. Eight of the nine established AMC services had a dedicated consult service staffed with addiction medicine physicians and one service was a part of the psychiatry consultation liaison service. AMC services varied in design, specifically the availability and coverage within the hospital, team composition, scope and responsibility of the service, and financing. The barriers and facilitators to AMC implementation, however, were similar across programs.

4.2.1. Availability and coverage. The $A M C$ services varied with access to resources and staffing, because of local supply-side attributes (e.g., financing and social structures). At the same time, many operational characteristics and challenges across the nine services were similar. Most established AMC services $(n=8)$ provided in-person consultation only during weekdays and a third of services $(n=3)$ provided consultation in the emergency department. The single program with weekend service availability was staffed by trainees who worked on the psychiatry consultation liaison service. Generally, the limited weekday availability frustrated key informants; they felt that patients who were admitted or discharged on weekends were not receiving life-saving addictionrelated services. As a result, care was sometimes rationed on the weekdays because of the weekend-related staffing limitations: 
The counselors came in with 26 consult orders yesterday, on a Monday, so they don't have time to do the assessments and to help actually find treatment beds for patients. So, they have to prioritize it to the highest needs, people who are directly transferring to treatment. (I1)

Key informants linked staffing constraints to limited financial resources. Staffing deficits led to work-around clinical processes, such as taking home call or consultations via pager. These approaches, however, did not mitigate the heavy patient caseload on Monday mornings. Limited financing for clinical staff also constrained physically where AMC consultation could occur (e.g., the emergency department). Key informants believed that to be a fully operational consult service it was essential to provide services in the emergency department.

4.2.2. Home department and team composition. The home department and AMC service team composition varied, reflecting local social structures within each hospital. In some programs, the AMC service was established through the departments of family medicine or internal medicine, and in one hospital, the AMC service based in the psychiatry and medicine departments. Eight of the nine established AMC services were staffed with addiction medicine physicians, although physician staffing resources varied. One well-established consult service had over 10 board-certified addiction medicine physicians; in contrast, others were limited to one physician. Eight of the nine consult services comprised of interprofessional teams. The most common non-physician team members were nurse practitioners $(n=4)$ and social workers $(n=4)$ and less common were alcohol and drug counselors $(n=2)$, physician assistants $(n=2)$, psychologists $(n=2)$, and peer support providers $(n=1)$, see Table 4.2 . 
Although not commonly a part of the AMC services, informants sought to add peer support providers (i.e., trained persons with lived addiction experience; "peers") to the team. At the time of interview, only one hospital had peers on their service. The primary responsibilities of the peer were to "...help patients stay in the hospital and help engage them" (deidentified ${ }^{3}$ ) and to provide conflict de-escalation. The key informant shared that the outcome most important to peers, which differed from the rest of the care team, was: "relationship[s]" (deidentified). The informant noted that the inclusion of peers on the team made other clinical staff in the hospital uncomfortable. The informant posited that this was because peers challenged the traditional hierarchy of clinical roles in the hospital.

In contrast to peers, medical trainees (e.g., addiction medicine fellows, resident physicians, and medical students) were common AMC service team members.

Informants reported that trainees were a critical resource for both the standard work of the service (e.g., trainees see the patient first) and as a mechanism for extending their clinical staffing resources (e.g., fellows staff the consult service).

Table 4.2. Addiction Medicine Consult Service Design $(n=9)$

\begin{tabular}{|c|c|c|c|c|c|c|c|}
\hline \multicolumn{2}{|c|}{ Availability \& Coverage } & \multicolumn{6}{|c|}{ Non-Physician Team Members } \\
\hline Weekend & ED & NP & Peer & $\begin{array}{l}\text { Physician } \\
\text { Assistant }\end{array}$ & Psychologist & $\begin{array}{c}\text { Social } \\
\text { Worker }\end{array}$ & $\begin{array}{c}\text { Alcohol \& Drug } \\
\text { Counselors }\end{array}$ \\
\hline $11 \%$ & $38 \%$ & $50 \%$ & $11 \%$ & $25 \%$ & $25 \%$ & $50 \%$ & $25 \%$ \\
\hline$(n=1)$ & $(n=3)$ & $(n=4)$ & $(n=1)$ & $(n=2)$ & $(n=2)$ & $(n=4)$ & $(n=2)$ \\
\hline
\end{tabular}

\footnotetext{
${ }^{3}$ Since this was a unique property, the attribution was deidentified.
} 
4.2.3. Responsibilities and scope of practice. AMC service responsibility and scope of practice existed in three categories: 1) the provision of SUD-related education to trainees, providers, and hospital staff; 2) the delivery of psychosocial and medical services (e.g., motivational interviewing, pharmacotherapy, linkage to care); and 3) hospital guidance document development (e.g., order sets, guidelines, and protocols).

4.2.3a. Education. Hospital-wide education related to SUD treatment was an important responsibility of the AMC service:

We teach an addiction mini-course in the second-year medical student curriculum. It is a required course at our university. It is not a long course...but we are one of the few medical schools who do that from my understanding...[our] residential treatment facility and detox center...is a clinical training site for third year medical students, as well as psychiatry residents... we have a Project Echo... and we do a lot of addiction medicine education to primary care, psychiatrists, case managers and counselors. (M1)

Further, the AMC services sought to inspire hospital culture change through their educational initiatives. The presence of the AMC service, moreover, enhanced understanding among hospital staff about the need to address OUD within the medical context. Informants perceived that the AMC service provided other benefits such as decreasing provider burnout, decreasing provider stigma, and improving the culture and the clinical practice for SUDs: "By having the right teams in place in the hospital we can really change the hospital experience not just for patients but for the providers" (A2) and "I think there is a morale booster, both for us and our colleagues. And then it just raises the profile of addiction in general when there is a consult service identified with it" (K1). 
4.2.3b. Service delivery. Generally, AMC services were responsible for five core services: 1) mental health and SUD assessments; 2) psychosocial intervention; 3) the medical management of SUDs (e.g., clinical activities related to OAT initiation, continuation, and discontinuation, the management of benzodiazepine and alcohol withdrawal); 4) the medical management of pain; and 5) linkage to care (e.g., referrals to treatment, bridge scripts, care pathways). Variation in team composition was associated with the different psychosocial techniques used by the service (i.e., brief intervention; cognitive behavioral therapy; dialectal behavioral therapy; and motivational interviewing) and harm reduction interventions (i.e., naloxone kit distribution; overdose education; counseling on syringe exchange; and allowing patients to smoke).

Informants emphasized that linkage to care was an essential and distinctive AMC service responsibility and they described four linkage to care modalities: 1) writing a bridge script; 2) referring to community-based services; 3) admitting to a transition program; and 4) transferring to another health system's bridge clinic. Bridge scripts were the most common linkage approach and occurred when an inpatient physician wrote a prescription for buprenorphine/naloxone to "bridge" the patient until they established care with a community-based provider. Other linkage to care approaches included providing referral to community-based services (e.g., direct transitions into residential treatment), which was primarily the responsibility of the case manager and social workers. 
Other less commonly used linkage approaches were the use of transition programs and bridge clinics. Informants described transition programs as "rapid access" and "reach-in" interventions, meaning that a community-based provider visited patients during hospitalization to prepare them for direct discharge to their outpatient program. Further, only one hospital had an operational bridge clinic at the time of interview. The bridge clinic transitioned patients, over a two-week window, to an outpatient community-based OAT provider. Both types of programs, transition and bridge clinics, were developed in collaboration with external partners.

AMC service standard work was hospital specific; however, two services shared a similar consultation process based on the different types of providers on the team. At their hospitals, staff could request either a consult from the service's behavioral health provider (e.g., social worker) or from a clinical provider (e.g., physician).

4.2.3c. Policy development. The final category of AMC service responsibility, led by physicians on the team, was the creation of hospital guidance documents; specifically, the creation and implementation of order sets, guidelines, and protocols related hospital-based services for patients with OUD and other SUDs. Hospital guidance document creation was a common responsibility and the content of those polices is explored and described further in Section 4.7 of this chapter. Typically, the AMC service leadership worked closely with pharmacy and therapeutics (P\&T) committee to ensure that OAT was available on the hospital formulary and that standard procedures were in place for the hospital OAT delivery: "The addiction consult service took the lead in 
partnering with the P\&T committee to make sure we had access to buprenorphine, extended-release naltrexone, and methadone, on the floors. [We] wrote protocols for how those medications are used" (A1).

4.2.4. Financing. A common concern and activity across all services was planning for and acquiring financial resources, which consistently constrained or facilitated AMC service existence and operation. The fiscal environment for the services was complex and tumultuous. Informants described service financial solvency dependent on a patchwork of monetary streams, including: in-house financing; third-party payer revenue; grant funding; state funding; and fellowship program funding.

Many services received in-house funding for the salaries of the clinical staff and salaries for the addiction medicine fellows. The services procured these funds from either a specific department (e.g., medicine or psychiatry) or from hospital operations. In addition, some operating funds came through third-party payer billing. One informant stated that they billed "just like any other consultant" (L1) and, other informants were acutely aware of their reimbursement return rates: "We also do our own billing for patients...[our] reimbursement return rate is only about $25 \%$ though, because the vast majority of patients that we see are Medicaid or uninsured" (J1) and "Our recovery rate was $28 \%$, so we get paid about $28 \%$ of what we bill... When we add it all up, if a nurse is billing at the right volume they actually pay for a lot of their salary" (K1).

Some AMC services operated as pilot programs through grant funding from the hospital or an external entity (e.g., third-party payer or the state). One informant shared 
how a local managed health care organization was funding the salary, for a limited duration of time, of specific service team members - the physician assistant and alcohol and drug counselor. Informants shared that state funding for the service came from state health care reform funds (e.g., accountable care organizations) or dedicated indigent funds in the states that opted out of Medicaid expansion.

4.2.5. Summary. To date, prior research has not described the shared and different organizational elements of more than one AMC service. Common design and operational practices included weekday only service availability, the use of interprofessional teams, the use of medical trainees, and three domains of responsibility (i.e., education, delivery of psychosocial and medical services, and policy development). AMC services varied by home department, the professional composition of the team, day-to-day operations, and how the services were financed.

These analyses suggest three important conclusions. First, AMC service responsibilities included two non-medical domains: 1) education; and 2) policy development. This finding is not surprising because of the interprofessional nature of the AMC service and because of the complexity of the external treatment environment (e.g., policies and regulations). Second, the AMC service was not only a mechanism for OAT delivery, but an intervention for shaping and changing hospitals' social structures through diverse responsibilities (i.e., education and policy development). Third, two supply-side attributes influenced and mediated the AMC service design and operationthe social structures within and outside the hospital environment, and financing. 


\section{Section 4.3. Facilitators for AMC Service Implementation}

Prior to this study, little was known about the facilitative attributes for the creation of hospital-based addiction technologies and delivery of care for patients with OUD. This section explores research sub-question $1 \mathrm{~b}$ : Which internal and external environmental supply-side elements facilitate hospital OAT delivery? A primary finding from the qualitative aim of this study was that the AMC service was a key mechanism for hospital OAT delivery and other OUD-related services. The service promoted and increased the use of evidence-based services in the hospital and also catalyzed the development of other organizational technologies, such as standard practice documents. This section describes the hospital and environmental attributes that facilitated AMC service establishment in 9 hospitals $(n=10)$ and the soon to be established services in the 5 hospitals $(n=5)$. These elements existed within and outside the hospital environment and as demand-side attributes (i.e., patient characteristics).

4.3.1. Facilitative attributes within the hospital. In the hospital environment, AMC service development was only possible within the context of positive social structures and appropriate technology and resources (i.e., staffing). Evidence of positive social structures included: 1) a history of caring for persons with addiction within that hospital or health system; 2) designated financial and clinical resources for addiction treatment; 3) addiction medicine clinical training programs; 4) normative treatment practices; 5) supportive clinical and administrative leadership; and 6) supportive internal stakeholders. 
4.3.1a. Historical support and practice. Informants noted that an institutional history of addiction related initiatives facilitated AMC service design and operations. Informants described prior practice as positively contributing to contemporary care delivery environments: "I worked in the hospital where one of the founders of Alcoholics Anonymous worked so the hospital has a long history of detox and treatment of substance use disorder (L1)" and "A neurologist who had become interested in addiction in the 1990s established a consult service and then there was an internal medicine doctor who took it over...it was in many ways...almost like a labor of love" (F1). Informants described three types of prior clinical practices that influenced service establishment: 1) ad hoc consults; 2) "curbside consults" (M1); and 3) a buprenorphine pager. Ad hoc consultation was the most common, present in several hospitals prior to AMC service existence. This type of practice occurred when an addiction medicine physician provided an informal consult on a SUD patient as a favor to a colleague:

One of my colleagues and I started doing ad hoc addiction medicine consultations five years ago and that grew into the AMC service... We had so many patients on the internal medicine and surgical wards with addiction issues that were really going untreated, people knew that my colleague and I prescribed buprenorphine and so they thought that we might be able to help...they would call us and we would leave whatever we were doing and go do a consult. (A1)

The "curbside consult" approach, used in one hospital, occurred when an addiction medicine physician provided informal consultation for the psychiatry consult service:

Basically, I just assist in education and curbside consult for the psychiatry consult team. I am not billing for consults myself the psychiatry consult team is, but if he needs my help he phones me and I provide him with, what I call a curbside consult. (M1) 
The third approach, at another hospital, was a dedicated buprenorphine pager staffed by a family medicine inpatient team that provided remote support on buprenorphine initiation. The informant shared that "we kind of just do that on our time on the side, to help coach providers who are interested in prescribing buprenorphine but aren't sure how to for their hospitalized patients" (C1). The guiding philosophy across all three strategies was the dedication of the addiction medicine physicians to the ethical treatment and care for people with SUDs in the hospital, regardless of compensation.

4.3.1b. Financial and clinical resources. Financial and clinical resources were essential to AMC service establishment. Existing treatment services and research programming for addiction often facilitated the creation of a service. From a clinical perspective, in hospitals with addiction-related services already in existence, clinical leaders could argue that AMC service creation was filling a gap in the SUD care continuum. These additional services differed among hospitals and health systems, but included established outpatient addiction medicine clinics, dedicated hospital units for managing SUD withdrawal, intensive outpatient programming, affiliated or onsite OTPS, and a residential halfway house. One informant commented that because of the already in existence robust institutional addiction research program that hospital leadership viewed addiction-issues with familiarity and positivity. In addition to physical resources, such as clinics, dedicated beds, and research programs, informants described the importance of the presence of human resources; specifically, addiction medicine 
physicians. Some of the informants shared that they were recruited to their institution to start a service or fellowship program: "I was hired with the intent that I would build this consult service and get it up and running" (I1).

4.3.1c. Clinical training programs. Finally, unsurprisingly, addiction-related education programs and training activities facilitated an addiction-friendly hospital environment. Informants described how the financing for their addiction medicine fellowship was crucial for establishing a service. More than one informant described this explicit financial connection between the service and the fellowship:

We kind of put a pitch together where we would ask for funding for the fellowship slot and then use that for our consult service... the chair of the department of medicine agreed to fund a fellowship slot and half of a nurse and then we would staff it with attendings who would bill for services. (K1)

Trainee champions were another supportive element to service development.

Residents, in particular, became champions within the hospital for improving OUDrelated services:

The initial pilot program for buprenorphine induction in the hospital was the idea of residents. And they really have taken this on. And asked to be trained, to be Xwaivered...All of the residents are being trained in buprenorphine prescribing. It has been exciting to see how the educational aspect of this hospital has moved things forward for everyone not just the folks who are currently in training. (C1)

These findings compliment observations from the prior section on the important contributions of medical trainees for AMC service operation.

4.3.1d. Normative practice. AMC service development was facilitated by care standards for SUD services within the hospital and health system. One informant reflected on how the normative clinical practice (i.e., standard of care) of their hospital 
was that all providers from all services must "be able to provide methadone maintenance. It is just routine standard of care." (C1). At another hospital, this practice was a requirement for all trainees: "We require that all our incoming interns...during new intern orientation get buprenorphine waived" (01).

\subsection{1e. Administrative and clinical leadership. Supportive hospital}

administrators, with guidance from addiction medicine clinical champions, drove AMC service establishment. However, hospital administrators varied in their reasons for supporting the service. Some administrators believed that addiction was a medical and public health issue, some administrators had relevant clinical experience, but nearly all were perceived to be moved to action by the "business case." Addiction medicine physicians were key leaders in developing and presenting the business case to garner hospital administrative support. The approaches used to engage hospital administrators included producing data on service needs and demands, including: 1) how the hospital census had an elevated SUD prevalence; 2 ) how untreated SUD negatively impacts hospital finances; 3 ) how other hospitals are benefiting from service implementation; and 4) why the hospital addiction treatment gap is a care quality issue. Each of these approaches are addressed in turn.

4.3.1e.1. Elevated SUD prevalence. Addiction medicine leaders presented either national public health data or internal census data on the prevalence of SUD-related admissions to their hospital: "Between 30 and 50\% [of hospitalized patients] or more, 
have some co-occurring substance use problem and the inpatient [setting] is an

opportune time to try and tackle those problems" (E1). Informants shared:

We were able to collect data... we presented that to administration...they didn't have the awareness of the saturation of the problem and we were really able to get buy-in from the president of the university... We basically just showed them the data. (M1)

4.3.1e.2. Negative financial impacts. Addiction medicine leaders also presented data illustrating how patients with untreated SUD in the hospital had increased resource utilization and how that was negatively impacting hospital revenue. Informants noted that readmission penalties and increased lengths of stay were persuasive arguments for service establishment: "What we ended up doing was basically through that needs assessment building a pretty strong business case around length of stay reduction and also building a business case around readmission reductions" (A2). At one hospital, administrators were convinced to implement a service because untreated SUD patients had longer lengths of stay which was inhibiting patient flow between the emergency department to the inpatient wards: "The emergency department was getting a lot of pressure from the hospital to move patients quicker, lots of patients were lingering in the emergency department that should have either been discharged or been moved to beds on the floor" (01).

4.3.1e.3. Success at other hospitals. Addiction medicine champions strategically leveraged the success of other prestigious institutions that were already providing inpatient resources for the treatment of SUD: 
In addition to the data that we had collected, it was honestly some healthy competition. I was saying look [institution A] has one of these, [institution B] has one of these, [institution C] has one of these, New York City is creating a city-wide service through the health and hospitals program. It is really silly that we don't have one. That caught their ear and they were able to look at the data a little more. (01)

Another informant shared how the positive press at a competing hospital pressured hospital leadership to move more swiftly in implementing their AMC service: "The neighboring institution started a service...a year before we did...and you know they had positive press coverage... my hospital had fashioned itself as a leader in addiction and it really prodded them and was useful external pressure." (K1)

\subsection{1e.4. Care quality. Informants identified and shared with leaders how} untreated SUD in the hospital setting was a care quality issue and why this issue fell under hospital purview. One informant stated that they "had to show that the volume of the service is really high [if] we are going to justify it [the AMC service]" (K1). Only one informant described presenting patient care preferences to hospital administrators when making the AMC service business case.

4.3.1f. Stakeholders. Internal stakeholders also supported AMC service establishment (e.g., physician-based consultation services, nursing, hospital security, and the P\&T committee). Informants affiliated with large academic health centers had access to other consult services. At some of the hospitals these other internal services were either supportive or were an impediment to AMC service formation. The supportive services varied and included the psychiatry consultation service, the pain consultation service, and the cardiovascular surgical service. Long-standing partnerships 
with psychiatric consultation services were common and the services actively worked together to delineate responsibilities, to collaborate on care for shared patients, and produce educational activities. One informant described an uncommon collaboratorthe cardiovascular surgical service. The surgical service became involved in the development of the AMC service at one hospital because the surgeons had decided that they were no longer going to conduct valve transplants on patients who injected drugs. The surgeons' refusal to care for these patients resulted in a joint medical ethics conference with the addiction medicine physicians, and this event inspired the surgeons to become vocal supporters of improving care for patients with OUD, they helped to lobby hospital administrators to launch the service.

Another informant shared the importance of working with hospital security staff and officers, to help them better understand SUDs, because many of the service's patients were either currently involved with the criminal-justice system or were participating in illegal activities while hospitalized:

The consult service slowly over time has been able to navigate that conversation with hospital security, and use more conflict de-escalation approaches and behavioral agreements to managing some of those behaviors with the goal of keeping people in the hospital to complete their treatment and then engaging them in the community for treatment afterwards rather than transitioning to jail. (A1)

Internal collaborators (e.g., hospital administrators, the P\&T committee, nursing staff, clinicians in other departments) further bolstered the creation of other hospitalbased technologies to promote evidence-based delivery of services for patients with OUD, and the development of hospital guidance documents: "It has been helpful to 
have support from multiple different department. The support of the obstetrics team, our anesthesiology team, and our pharmacy" (C1).

4.3.2. Facilitative attributes outside the hospital. The external environment, outside the hospital, positively mediated AMC service creation. Facilitative environmental influencers included: 1) non-clinical stakeholders; 2) clinical stakeholders; 3) a robust community-based treatment network; 4) media coverage on the opioid overdose epidemic; 5) policies and regulations; 6) politics and political action; and 7) financing.

4.3.2a. Non-clinical stakeholders. Non-clinical stakeholders contributed to the introduction of and support for AMC services. Stakeholders helped by: 1) generating new normative expectations and behaviors related to hospital addiction care; and 2) pressuring hospital leadership to address the issue. The non-clinical organizations of influence included the Association of American Medical Colleges (AAMC), the Office of National Drug Control Policy (ONDCP), local governments, and the state Medicaid programs.

4.3.2b. Clinical stakeholders. Key informants recognized the value of formal and informal partnerships with community-based clinical providers and other local institutions. One informant noted that: "There is no doubt that without pathways to treatment in the community we would be hamstrung" (A2). Informants described professional relationships with other hospitals in their community and with a peer- 
based agency. Support from colleagues at a nearby hospital facilitated service training and development:

We are very friendly with them [the providers at another hospital] .... Our clinics talk together the most...more than our consult services, but the...clinics are really extensions of the consult services... The other institution is starting a fellowship so we plan on including them in our multiprogram case conference... We are all friends. (K1)

4.3.2c. Robust community-based treatment network. Access to communitybased treatment resources facilitated AMC service establishment in some communities. Although the majority of informants indicated a dearth of community-based treatment services available to their patients, a few informants perceived that their communitybased treatment network was readily accessible for their patients upon discharge:

In our state, different counties have mental health and recovery boards and funding to support treatment for people, and the county where we are located is very very good, they have a lot of treatment facilities and options where they pay for people to go to treatment. So, they were very helpful to us because they offered us pipeline warm hand off essentially for people who were seen in consultation to be able to transition after they got out. (L1)

4.3.2d. Awareness generation through media coverage. Public awareness of the opioid overdose epidemic, occurring primarily through local and national media coverage, supported service creation. General awareness about the opioid overdose crisis among hospital leadership created pressure for leadership to start a service. An informant stated that: "What really opened up the door was the opioid epidemic. Now hospital leaders have been paying attention" (E1), and another noted that they believed "it has been all the attention on the opioid crisis has been getting in popular media and dollars are starting to be direct to that" (J1). Further, local media coverage educated 
other providers within the hospital about the challenges of the opioid overdose epidemic in their local community: "I think all of the news media coverage of the opioid epidemic. Our providers are seeing the reality of that on the streets of our city. It has been hugely helpful" (C1).

4.3.2e. Policies and regulations. External policies and regulations generally were predominantly unsupportive of AMC service establishment. Despite this, informants noted four external policies and regulations that helped: 1) the American College of Surgeons' trauma certification requirements; 2) Medicaid expansion; 3) the changing telehealth laws; and 4) Medicaid coverage for SUD treatment. One informant elaborated further:

One big positive is our state signed up for Medicaid expansion. A lot of these patients are covered [by Medicaid] ... When I talk to people in North Carolina, they don't have that. It is hard for me to imagine you know how they can help patients. In that regard we are fortunate that many of our patients can access health insurance and that provides them with access to good treatment. (D1)

4.3.2f. Supportive politics and political leadership. Local political leaders contributed to AMC service implementation. In some jurisdictions, local city leadership explicitly called upon hospitals to act. One informant reported that: "The health commissioner here has encouraged hospitals to get more involved" (D1). In a different city, the leadership of the city health department scaled up the city-wide hospital opioid overdose prevention programs and provided funding for naloxone distribution in hospitals. 
4.3.2g. Financing. Overall, the financial landscape constrained AMC service implementation and operations, however, in certain contexts, financing promoted service formation. One informant shared how quality metrics and financial incentives and penalties linked to hospital benchmarks helped to support the business case for the existence of an AMC service. In some local contexts, state and regional capitation-based insurance programs and their payment policies tied to readmission rates indirectly drove hospitals to improve services for persons with SUDs, who were commonly readmitted.

4.3.3. Summary. The synthesis of these findings helps to answer why a simple supply-demand model is insufficient to explain AMC service development: "It is [the] convergence of funding opportunities...greater awareness, and grassroots advocacy by me and my colleagues here at the institution...." (E1). These analyses suggest that the contributions of internal and external supply-side attributes were influential for inspiring hospital innovation and creation of new care delivery mechanisms for persons with OUD and SUD. Support attributes within the hospital context included positive social structures for addiction-related services, historical practice, previous clinical and resource investments in addiction related care, especially addiction medicine training programs, normative treatment practices, and supportive internal stakeholders. Partnerships with clinical and non-clinical stakeholders further supported service development. Additional facilitators included access to community-based treatment 
providers, normative practices related to addiction treatment, policies and regulations, financing, and media coverage and awareness of the problem.

\section{Section 4.4. AMC Service Implementation Barriers}

This next section explores research sub-question 1c: Which internal and external environmental supply-side elements impede hospital OAT delivery? Informants from all 16 hospitals $(n=17)$ furthered the understanding of barriers to AMC service development and hospital OAT delivery. These constraining elements existed within and outside the hospital, providing insight as to: 1) why hospital AMC services are a relative new mechanism of care; 2 ) why adoption of AMC services across the U.S. is slow; 3 ) why the hospital OAT delivery is underused; and 4) the potential leverage points to decrease barriers.

4.4.1. Barriers within the hospital. Key informants from all 16 hospitals described internal barriers to service establishment, service planning, and the delivery of OUD-related services. Six interdependent organizational barriers emerged: 1) restrictive policies and regulations; 2) existence of bureaucratic processes; 3) limited hospital-based staffing and resources related to addiction service delivery; 4) internal stakeholders; 5) stigmatizing behavior and training gaps perpetuated by hospital staff and administration; and 6) financial instability.

4.4.1a. Restrictive policies and regulations. In some hospitals, the inpatient pharmacy and P\&T committee would create internal policies that inhibited OAT access: "There are certain rules you have to follow for using certain medications within the 
hospital" (L1). One hospital did not have mono-product buprenorphine and only stocked combo-product (buprenorphine/naloxone), thereby creating issues for treating pregnant women because first-line treatment is mono-product. In another hospital, conversely, the hospital formulary only included the mono-product buprenorphine, requiring patients using the buprenorphine/naloxone combination product to switch or discontinue during the admission. Another informant gave up trying to get the buprenorphine implant added to the hospital formulary, stating that: "[the hospital] will road block anything" (G1). Other internal formulary and prescribing restrictions included limitations on when providers could prescribe buprenorphine/naloxone. One hospital, for example, restricted buprenorphine/naloxone administration only to prescribers with a federal buprenorphine waiver. Notably, this was one of the two hospitals without an AMC service or plans to start one.

4.4.1b. Bureaucratic processes. Barriers to service development were related to the bureaucratic properties of large hospitals: size, staff turnover, and slow and complex decision-making. The large size of teaching hospitals created challenges for service development because the newly forming AMC service was just one of many consultation services: "[there are] only so many hours in the day for you to get in front of people's eyes" (H1). Further, more than one informant described staff turnover as operationally problematic, creating confusion related to the service's roles and responsibilities. Finally, the slow, formal, and hierarchal decision-making process slowed down service development: "There are always the process barriers in any kind of 
hospital system or university. If you present a procedure it has to go through formal

processes, formal committees, policy committee, and up to the top" (M1), and:

As far as rolling something out you got to get it cleared by a million different people, there is like, it is like dealing with the federal government basically, you can't just get everyone in a room really quick and here's what we are going to do. Our size works against us. (H1)

4.4.1c. Limited staffing and resources. Limited clinical staff with addiction medicine expertise was a common barrier for service development and operations: "We haven't had enough qualified faculty to be able to [start the service] until recently" (N1). In the two hospitals without an AMC service or the plans to start one, informants shared that the primary cause was a lack of resources, stating that: "Manpower is number one" (G1) and "It is mostly been for lack of personnel... [to] have the bandwidth to do it" (B1). Even for those with an established AMC service, after launching, more than one informant described issues related to access to physical resources and staffing: "I still have no office. I still have no dedicated land line. I don't have an admin" (P1). When asked about dedicated individual time to the AMC service one key informant responded: "I have about 20\% FTE which is not nearly enough" (J1).

4.4.1d. Unsupportive internal stakeholders. Another common barrier to service development and operations was resistance from other hospital stakeholders. An informant noted: "There...[are] certain individuals who are going to slow that process down...They have some personal biases" (M1). Psychiatry consultation services were frequently responsible for creating roadblocks. More than one informant described the tension between the two services as a "turf war." Some opined that the AMC service 
threatened the psychiatric consultation liaison service because of the other service's low volume: "Ironically it is the psychiatrists here who aren't as enthusiastic. It has been kind of a funny turf war. People get their thing going at a hospital and they see other services as competition" (E1). Informants also believed that the psychiatrists were reluctant to deliver evidence-based OUD services because of stigma and limited training experience in caring for persons with SUDs.

Hospital leadership also impeded service and addiction treatment resource development. One informant, without a service, described how leaders at their hospital had "no foresight" (G1) when it came to issues related to addiction. This informant believed that unless outside forces intervened, such as an external mandate requiring evidence-based service delivery for OUD, or for reimbursement, that hospital leadership would never address the issue. Hospital leaders also had concerns about starting a service without explicitly identified connections to care upon discharge:

I think there was also a lot of concern from our hospital leadership around making sure that patients were connected to outpatient care on discharge. Which is a complex issue because there are some patients who may or may not, as I was saying, connect to care on discharge. (C1)

4.4.1e. Stigmatizing provider behavior and training gaps. A consistent barrier to hospital OAT delivery across hospitals was a lack of provider education and training. This resulted in stigmatizing behaviors and discriminatory practices by non-addiction trained hospital providers: 
Stigma is about lack of education... and the personal experience of the person who is stigmatizing the patient...I encourage [other clinical providers] to understand [addiction] through the chronic disease model and [I] just listen... Many times, it turns out...it is a lack of information and once I provide information the stigma sort of just melts [away]. (P1)

Limited education and training on the treatment of SUDs is based on historic and contemporary social structures within undergraduate and graduate medical education training programs and further enforced through other supply-side attributes (e.g., reimbursement and financing policies, undergraduate and graduate medical education accrediting bodies). The confluence of these elements informed hospital culturalcognitive social structures and in turn, likely negatively impacted the care of persons with OUD and other SUDs within the hospital. Informants frequently observed stigmatizing and discriminatory behavior by other providers and recognized that these actions reflected the failure of medical education to train physicians properly in addiction:

Historically, the medical profession really has not viewed the treatment of addiction as part of what they do. At best, people would get a referral at the end of their hospitalization for rehab. And there would be no attempts to initiate treatment within the hospital. Obviously, that has not worked very well. Even when the primary reason for hospitalization was related to their substance use. (A1)

Further, informants observed other hospital providers as having historically antiquated and uninformed ideas about addiction:

In this area of the country there is still the perception by many people that addiction is not an illness, but it is a spiritual deficit, or a personal, or personality deficit. We have a lot of barriers just in terms of educating our staff to not treat these people differently than they would treat an individual who comes in with chest pain. (M1) 
Even more alarming was the observation that providers who interfaced most frequently with SUD patients, orthopedic surgeons and emergency department physicians, were particularly uninformed on OUD and SUD treatment best practices. An informant described how the orthopedic surgical team had a culture of "just treat 'em and street 'em" (H1). In the emergency department, one informant shared how she believed that emergency services were re-traumatizing SUD patients and that it was a missed opportunity for care. Further, another informant explained that emergency department services were deficient because of "Ignorance...you know the kind that many of us in the health care field have been raised with. Modeling is often...not often very consistent...and non-therapeutic" (G1). This informant perceived that the origin of the challenges in the emergency department was due to the department's externally contracted physician staffing model that resulted in limited incentives to improve care. Another informant shared how emergency department physicians at their hospital believed it to be illegal to provide methadone for withdrawal: "I am going to talk at the [emergency] department meeting and explain that it is in fact legal and humane to treat withdrawal and that is how you can do it safely. There are really big gaps in knowledge" $(\mathrm{A} 2)$.

Provider misunderstanding and confusion regarding OAT policies and regulations was common across all hospitals, and not only an issue specific to the emergency department providers. Informants frequently observed non-addiction trained providers 
misinterpreting OAT regulations and subsequently not providing care. Providers and administrators believed it was illegal to administer OAT in the hospital:

There is still the perception on the part of many providers that somehow it is illegal to engage in that practice [administer methadone in the hospital] .... [the] pharmacy has been a barrier, when Suboxone ${ }^{\circledR}$ or methadone-based maintenance and taper regimen gets ordered and the pharmacist says you can't do that, that is illegal. (J1)

I know that because we are not certified as an OTP that there is much hesitance around this [administering methadone]. I know that there is as you know, the 72hour rule, so you can manage withdrawal on inpatients, but it is not practiced. I would say that generally our legal department is very conservative when it comes to things like that. (B1)

Hospital providers, not trained in addiction medicine, often believed they had to have a special wavier to administer OAT during hospitalization: "The perception is that a provider making the order, even in the hospital setting, needs to have the DATA waiver to prescribe administer a buprenorphine product for that indication" (J1). Beyond legal confusion, informants observed that other providers had a limited understanding of OAT efficacy. It was common in one hospital that non-addiction trained providers believed that OAT was "substituting one drug for another" (C1).

In addition to the confusion about legality and efficacy, informants observed more subtle forms of stigma-apathy. This was most commonly perpetuated by hospital leadership who decided against supporting or investing AMC service resources:

They start with all the reasons it can't be done and stop there...you know people don't want to say out loud things like "well we don't to be known for that kind of thing. "I think again that maybe they feel we don't want to draw attention to that I would rather draw attention to our new cardiology center...so its prejudiced. (G1) 
Hospital leadership also held AMC services to a higher productivity standard than

other services. An informant noted the hypocrisy of how the AMC service had to justify

its existence by proving financial sustainability, while other non-revenue generating

services continued to operate without the that expectation:

I think there is still a lot of embedded stigma. It is structural. To assume that there is a good reason for why the addiction consult service is held to a different standard than established consult services than you know... we are only going to do it if it is good for our business. Number one public health issue that the community is facing. Ebola didn't generate a lot of revenue but it cost a shit load for the hospital even though we didn't have one single patient. (K1)

Finally, and importantly, an informant observed that language used within the

addiction medicine community may perpetuate stigma and mythology around the

complexity of the medical management of OUD:

The term induction is something that I don't really like a lot. I don't actually use it myself. I just say I am starting treatment. It makes it seem like this fancy procedure that we are doing and we are just starting someone on treatment. I don't really see it as any different than starting any other treatment... I think calling it induction is a barrier to starting treatment. (D1)

4.4.1f. Limited internal financing. Securing and sustaining internal financing for

the AMC service was the "the biggest barrier" (A1) and "the hardest thing" (M1). The complex financial landscape created multiple barriers to planning, establishing, and delivering inpatient evidence-based AMC services. First, most AMC services operated from a cost savings model versus a revenue-generating model, making it challenging to articulate the fiscal value of the service to hospital leadership: "No matter what...we operate at a loss...there is not enough billing, our billing does not support the breadth of our team" (A2). Respondents from two different hospitals stated that their hospitals had 
closed their previous AMC services because of funding issues, because of these internal constraints, informants had to look outside the hospital for funding:

We are currently applying for grant funding to do a pilot. To get a couple [of] years' worth of funding to attempt to have a consult service with the hope that the hospital will see that it is useful to them and take over funding in the future. (C1)

4.4.2. Barriers outside the hospital. Key informants described four external supply-side constraints to establishing an AMC service and for delivering OAT: 1) limited access to community-based treatment resources; 2 ) restrictive and limiting policies and regulations; 3) unsupportive local politics; and 4) unstable financing.

4.4.2a. Limited community-based treatment network. Informants identified that a limited community-based treatment network constrained service delivery and design within the hospital because of issues related to hospital discharge and transitions to care. Informants described five barriers that could prevent a patient from successfully transitioning: 1) the location of the resources or facilities in relation to where the patient lives; 2 ) the capacity of the treatment programs (e.g., number of beds, number buprenorphine providers, number of clinicians); 3) discriminatory organizational practices and policies; 4) insurance barriers (e.g. programs not accepting Medicaid); and 5) a limited range of programs with the appropriate intensity of services.

The community-based treatment programs such as skilled nursing facilities and social service and housing programs used discriminatory practices and policies to deny admission to patients discharged from the hospital on OAT. These policies and practices commonly hinged on the issue of the patient receiving OAT at discharge and the facility 
not wanting or having the resources to support the patient in continuing their OAT medication. One informant shared that the skilled nursing facilities in her community always happened to be full whenever their team tried to discharge a patient there who had history of injection drug use. Another informant observed that treatment centers were unwilling to invest in resources to care for persons with intravenous antibiotic needs, resulting in patients "sitting here in the hospital for 6 weeks because no treatment center is able to take someone with a PICC line..." (I1).

4.4.2b. Restrictive policies and regulations. Additional challenges for delivering evidence-based services to persons with OUD in the hospital setting were related to policies and regulations created and enforced by non-governmental (e.g., Accreditation Council for Graduate Medical Education [ACGME]) and governmental (e.g., local, state, and federal) bodies. Many of the described regulatory barriers were connected to OTPs. One informant shared how state-mandated clinical assessments that had to be completed prior to OTP placement limited the number of patients that the service could see because they did not have enough staff to complete the multi-hour assessments for all qualified patients. Further, federal regulations allowed OTPs to create their own organizational policies, which can result in discriminatory practice. An OTP in one community, for example, discharged patients from its treatment program if the patient had a positive urine drug screen for methamphetamine. Moreover, informants continued to be frustrated by the federally mandated buprenorphine patient panel 
limits and the strict regulations imposed by pharmacy boards, the DEA, and third-party payers.

Forthcoming changes (academic year 2019) to educational policies may also constrain hospital-based OAT delivery. Recently, some addiction medicine fellowship programs were approved for accreditation through the ACGME. ACGME fellows, however, are not allowed to bill for patient visits independently, in turn likely changing the supervision and staffing requirements of some of the AMC services.

4.4.2c. Unsupportive politics and political leadership. Key informants discussed the challenges of delivering evidence-based services in the hospital setting because of an unsupportive political environment: "It is all personality, logistics, and politics honestly" (M1). An informant practicing in a politically conservative community described OAT barriers as existing because of a lack of political will: "We are at the mercy of the legislature here... and so you know they are willing to spend money on the unborn but not on anybody at birth unfortunately...we are trying to make headway there" (N1). Further, political leaders in this jurisdiction had other uninformed opinions about evidence-based OAT:

[The state Medicaid program does not pay for methadone]. People who are on methadone have to pay private facilities cash in order to get methadone... I can speculate but I think it is political. There is this stigma. They think these people are enjoying themselves on methadone. They do not see it as therapeutic. (N1)

4.4.2d. Limited financing. Informants identified multiple financial barriers to delivering hospital OAT: 
I think really the biggest obstacle is the money. You just got to follow the money. If you just follow the money. I think people culturally are willing to accept that addiction is a disease and to treat it is such. But they aren't going to do it out of the goodness of their heart. You have to find a way to pay them. (E1)

Third-party payers imposed five external financial barriers for the AMC services:

1) a lack of reimbursement for interprofessional teams; 2) the incentivization of nonevidence-based services (e.g., funding detox beds); 3) reimbursement restrictions; 4) prior authorizations and utilization review; and 5) limited financial incentives for delivering evidence-based care. An informant noted that: "The hospital is not going to turn off that revenue stream [for detox beds] just because they don't think it is the best idea for the patient" (H1). A source of deep frustration among informants were the third-party payer pharmacy benefit management strategies for OAT such as prior authorizations, quantity limits, and formulary restrictions:

[Our state] requires essentially a prior authorization before basically anyone can write Suboxone ${ }^{\circledast}$, and each insurance company, and even each Medicaid plan. We have [multiple] managed Medicaid plans in the state. Each one has different guidelines on what you are allowed to write... and of course quantity limits... That is going to be a huge barrier to doing this in the emergency room. (B1)

If we induce them in the hospital...it is always unclear to me if the insurance company is going to approve the films or the tablets? Are they going to approve the tablet size? Are they going to have a problem with that? I end up re-writing prescriptions quite a bit in the hospital just to get the prior authorization approved for a couple of days. (11)

Another complicating financial element was third-party payer contracting. Most hospitals in the U.S. participate in the diagnostic related grouping (DRG) billing system, which is a contractually agreed upon payment for a specific condition or treatment bundle. This is problematic for treating OUD and SUDs because there is no DRG specific 
to the interprofessional and comprehensive management of drug use disorders secondary to the primary reason for admission; thus, hospitals cannot be rewarded for comprehensively caring for patients with SUDs under this contractual structure. One informant noted that billing barriers may also exist in the emergency department and were told by administrators that they were prohibited from billing consultation services in the emergency department. It is unclear if this is either a jurisdiction or hospital policy specific issue. Finally, key informants described issues related to pharmaceutical pricing as influencing accessibility to OAT in the hospital context. At one hospital, the hospital only purchased mono-product, and did not stock combination-product because of costs.

4.4.3. Summary. The external supply-side barriers to AMC service establishment and the delivery of evidence-based OUD services were extensive and prohibitive. The socio-economic-regulatory environment in which hospitals exist was described as inhospitable for AMC service establishment and for the effective delivery of OAT. Barriers within the hospital setting included policies and regulations, bureaucratic processes, limited staffing and resources, internal stakeholders, stigmatizing provider behavior and training gaps, and limited financial resources. In the external setting, the barriers were access to community-based resources upon discharge, external educational and care delivery policies and regulations, and third-party financing (e.g., billing and reimbursement). 


\section{Section 4.5. The Contribution of Demand-Side Attributes}

Although the focus of this research, in theory and design, was on the facilitative and constraining supply-side elements related to AMC service development and hospital OAT delivery, informants also described contributions to care from the demand-side (i.e., patient attributes). Informants perceived demand-side elements as primarily increasing barriers to evidence-based services. Patients with OUD who injected drugs were particularly challenging to serve in the hospital because of their complex medical and social sequalae including serious infections (e.g., endocarditis), concurrent stimulant use disorder, homelessness, criminal justice involvement, or a lack of health insurance. Insurance status and third-party payer coverage policies also created barriers to patients getting timely access to OAT or other OUD-related therapeutics:

Sometimes insurance can be an issue for patients getting rapid access. We may be able to give them extended release naltrexone while in the hospital, but to give that when they leave, it typically requires an onerous prior authorization process, we sometimes run into the same issue with buprenorphine and can result in gaps in treatment. (A1)

Finally, patients had their own internalized stigma about OAT:

Unfortunately, there are many patients that are not interested in maintenance treatment and so unfortunately a lot of people end up requesting tapers and they don't always appreciate the efficacy or understand the gravity of their problem...I think part of it is the stigma about medication assisted treatment. I think a lot of patients fear medication assisted treatment, they don't want to be "hooked" on something, they don't want to be dependent on something, and I think particularly around hospitalization many people view that as this important moment, I am finally going to get clean. Unfortunately, they view being on methadone or buprenorphine as not being clean. (H1) 
In contrast, the only facilitative demand-side attribute described by the informants was pregnancy. Pregnant women were a frequent focus of efforts for providing timely evidence-based protocol driven hospital-based services supported with linkage to care. This model population exemplified how people with SUDs could effectively receive care in the hospital: "Pregnancy was probably the place where all this was standardized first...anyone who presents to the emergency department pregnant and in withdrawal is supposed to come up the labor floor and if they want to, they get induced and stabilized" (F1). It is possible that the consistency around care for pregnant women with an OUD is primarily driven by the desire to decrease complications during pregnancy, in particular premature birth, and to improve outcomes when neonates are born to minimize the harms, and length of stay, as it relates to neonatal opioid withdrawal syndrome.

\section{Section 4.6. Beyond the AMC Service: Emergent Supply-Side Elements}

Beyond the development and implementation of AMC services, informants described six emergent supply-side actions to enhance OUD-related hospital services: 1) incentivize evidence-based care; 2 ) increase addiction-related training requirement; 3 ) create treatment organizations or programs to enhance continuity of care; 4) deliver care through a trauma-informed and harm reduction approaches; 5) reframe the opioid problem; and 6) review and improve treatment policies.

Informants recommended creating financial incentives for high-quality evidencebased SUD care and to disincentivize non-evidence-based care during hospitalization. 
Informants also recommended that third-party payers should not reimburse ineffective services, such as only offering opioid withdrawal services ("detox" protocols) and that third-party payers (i.e., CMS) should develop value-based reimbursement mechanisms to create external financial incentives:

I think if there were more clear incentives, financial incentives, in terms of reimbursement structure that would be the best way to mobilize doctors to pay attention to this problem and invest in making changes systematically to help people with substance use problems in the hospital. That is the type of thing that requires CMS to really take the lead. (E1)

Informants recognized education as an important element for improving hospital services for patient with OUD. An informant noted that one approach to increase the number of hospital AMC services across the U.S. could be to require AMC service rotation as a part of ACGME internal medicine accreditation.

Another suggestion to improve hospital services for patients with SUD was to create dedicated healing spaces for patients to go upon discharge, such as a skilled nursing facility with addiction medicine services. Informants reflected on the traumatization of hospitalization and the need for trauma-informed training among hospital staff: "I don't think I fully appreciate the amount trauma that hospitalization really...it is so traumatizing. I think. In the power and the hierarchy... it is really intense" (A2). Further, informants noted that internal hospital policies, such as a no smoking policy, were not effective for supporting people with SUDs during hospitalization:

There is a policy about smoking...People aren't allowed to smoke. They aren't allowed to even walk outside to go to smoke... There is no smoking allowed either in or around the hospital. Sometimes people want to leave to go smoke and sometimes they just go smoke. And that creates issues. (D1) 
One of the things I have had to learn. Is to prioritize our goals... You want to make sure somebody isn't going to die of a heroin [over] dose when they leave, you want to make sure they complete their antibiotic course for endocarditis. So, we really focus on those things. And sometimes that means letting smoking slide. If for example the thing that is keeping them in the hospital is going out and smoking every day, you let them go out and smoke. (A1)

Informants generally perceived that hospital leadership and, subsequently, hospital-based initiatives, overly focused on opioid delivery with not enough emphasis on OUD treatment. In general, hospital administrators and the broader non-clinical staff had narrowly constructed assumptions about the problem of opioid overdose and the responsibilities of the medical community to address the opioid overdose epidemic. The rationale was that if access to opioids is severed in the health care setting, people will stop using prescription opioids, and the opioid overdose epidemic will end. This type of rationale places opioids and opioid use in the category of deviant behavior, and discredits the clinical utility of opioids as an analgesic: "Just because they have an opioid use disorder does not mean we need to take all opioids away from them. We still need to adequately treat their pain and do it in as safe a way as possible" (C1).

Informants suggested that policymakers interested in enhancing SUD service delivery in the hospital, review the treatment policy barriers associated with transitions to care, particularly for OTPs, SNFs, and the criminal justice system. One potential intervention to improve hospital transitions to care, as discussed by informants, was to open hospital-affiliated OTPs. An informant, however, noted how challenging this would be: "The limitations with [OTPs] are massive. There are both state laws and city specific laws that render it almost impossible to find a physical space in which you could open a 
new OTP" (F1). Decreasing barriers to care through the elimination or revision of existing policies in the external setting could enhance the provision of hospital services.

Informants described a range of issues relevant to improving and enhancing the delivery of services for patients with SUD, and particular those with OUD. They recognized the power of money in the health system, calling for the development of reimbursement programs that financially reward hospitals for providing evidence-based services and penalizing hospitals that provide non-evidenced based services to patients with SUD. Informants noted the benefits and harms of addiction medicine specialization and also noted the constraints of community-based OUD treatment policies for delivering effective services for patients with OUD during hospitalization and upon discharge. Informants also described a need for creating community-based treatment programs to enhance continuity of care and the need to provide education to hospital providers on trauma-informed care. Finally, more broadly, informants articulated that hospital leadership had a narrow conceptualization of the opioid overdose epidemic and that reframing the issue around treatment could be a helpful approach for designing effective hospital-based services.

\section{Section 4.7. Hospital Guidance Documents and Standards of Care}

This next section explores research sub-question $1 \mathrm{~d}$ : How do hospital policies inform OAT delivery? This was assessed through the analysis of 25 OUD-treatment related hospital guidance documents (i.e., policies, procedures, protocols, guidelines, quick guides, and order sets) from 10 non-VHA hospitals (see Table 4.3), and informant 
interviews. Hospital guidance documents, a type of organizational technology, are both an output of addiction related technologies and resources within an organization (e.g., addition trained clinical staff) and a reflection of social structures within the hospital, specifically cultural-cognitive structures, which are the beliefs of people within an organization (Scott, 2003). The findings from this section provide a preliminary understanding of how hospital OAT delivery is conceptualized and potentially practiced in well-resourced, non-VHA hospitals; and supports prior findings that an output of the AMC service includes the development of other hospital technologies, such as hospital guidance documents.

4.7.1. Guidance document domains. Two overarching policy domains, comprised of eight policy sub-domains, were observed across guidance documents: 1) OAT management and 2) security and behavioral management (see Table 4.4). The most common policies were in the OAT management policy domain, specifically for opioid withdrawal $(n=6)$, and the second most common was OAT continuation $(n=4)$. Of the nine hospitals with an established AMC service, seven (Hospitals A, D, F, H, I, J, and K) provided documents related to the care of persons with OUD. Policies were not provided by Hospitals $E, G, L, M, O$, or $P$. The policy summaries (33 pages of text) may be requested from the primary researcher. 
Table 4.3. Hospital Guidance Documents: By Title

\begin{tabular}{|c|c|c|}
\hline Hospital & AMC Status & Guidance Document Titles \\
\hline A & Established & $\begin{array}{l}\text { 1. Preoperative and Postoperative Pain Management } \\
\text { 2. Practice Guideline } \\
\text { 3. Safety Agreement Policy } \\
\text { 4. PICC Policy } \\
\text { 5. PICC Assessment Tool }\end{array}$ \\
\hline $\mathrm{B}$ & None & 6. Aberrant Drug Use Policy \\
\hline $\mathrm{C}$ & Planned & $\begin{array}{l}\text { 7. Acute Pain Management Guideline } \\
\text { 8. Inpatient Methadone Guideline } \\
\text { 9. Inpatient Buprenorphine Guideline } \\
\text { 10. Methadone Order Set } \\
\text { 11. Buprenorphine Order Set } \\
\text { 12. Methadone Quick Reference Guide } \\
\text { 13. Buprenorphine Quick Reference Guide }\end{array}$ \\
\hline $\mathrm{D}$ & Established & $\begin{array}{l}\text { 14. Withdrawal Management Protocol } \\
\text { 15. Methadone Protocol }\end{array}$ \\
\hline $\mathrm{F}$ & Established & 16. Withdrawal Management Protocol \\
\hline $\mathrm{H}$ & Established & 17. Withdrawal Management Order Set \\
\hline I & Established & 18. OAT Guideline \\
\hline $\mathrm{J}$ & Established & $\begin{array}{l}\text { 19. Aberrant Drug Use Policy } \\
\text { 20. Withdrawal Management Order Set }\end{array}$ \\
\hline $\mathrm{K}$ & Established & $\begin{array}{l}\text { 21. Leaving Against Medical Advice Policy } \\
\text { 22. Preoperative and Postoperative Pain Guideline } \\
\text { 23. OAT Management During Pregnancy Guideline } \\
\text { 24. OAT Management in Non-Pregnant Adults Guideline }\end{array}$ \\
\hline $\mathrm{N}$ & Planned & 25. Withdrawal Management Protocol \\
\hline
\end{tabular}

Table 4.4. Hospital Guidance Documents: Content Areas ${ }^{\mathrm{a}}$

\begin{tabular}{|c|c|c|c|c|c|c|c|c|}
\hline \multirow[b]{2}{*}{ Hospital } & \multicolumn{4}{|c|}{ OAT Management } & \multicolumn{4}{|c|}{ Security and Behavioral Management } \\
\hline & $\begin{array}{l}\text { Acute } \\
\text { Pain }\end{array}$ & $\begin{array}{l}\text { OAT } \\
\text { Cont. }\end{array}$ & $\begin{array}{l}\text { OAT } \\
\text { Initiation }\end{array}$ & $\begin{array}{l}\text { Opioid } \\
\text { Withdrawal }\end{array}$ & $\begin{array}{l}\text { Aberrant } \\
\text { Drug Use }\end{array}$ & $A M A$ & Safety & $\begin{array}{l}\text { PICC } \\
\text { Line }\end{array}$ \\
\hline$A^{*}$ & $\mathrm{x}$ & . & . & . & . & $\mathrm{X}$ & $\mathrm{x}$ & $\mathrm{X}$ \\
\hline $\mathrm{B}^{1}$ & . & . & . & . & $\mathrm{x}$ & . & $\mathrm{x}$ & $\mathrm{X}$ \\
\hline $\mathrm{C}^{2}$ & $\mathrm{x}$ & $x$ & $\mathrm{x}$ & . & . & . & . & . \\
\hline$D^{*}$ & . & $x$ & . & $x$ & . & . & . & . \\
\hline $\mathrm{F}^{*}$ & . & $\cdot$ & . & $\mathrm{X}$ & . & . & . & . \\
\hline $\mathrm{H}^{*}$ & . & . & . & $\mathrm{X}$ & . & . & . & . \\
\hline$\left.\right|^{*}$ & . & $X$ & . & $X$ & . & . & . & . \\
\hline $\mathrm{J}^{*}$ & . & . & . & $x$ & $x$ & . & $x$ & . \\
\hline $\mathrm{K}^{*}$ & $x$ & $x$ & $x$ & . & . & $X$ & . & . \\
\hline $\mathrm{N}^{2}$ & . & . & . & $x$ & . & . & . & . \\
\hline
\end{tabular}

Table Notes. ${ }^{a}$ Guidance documents could be categorized into more than one policy domain; *Established AMC service; ${ }^{1}$ No AMC service without plans to start one; ${ }^{2} A M C$ service being planned; $A M A=$ leaving the hospital against medical advice; $P I C C=$ peripherally inserted central catheter; $O A T=$ opioid agonist therapy; Cont. = continuation. 
4.7.1. Domain 1: OAT management. Informants from all hospitals provided or described hospital-based policies and practices related to OAT management. Four OAT policy and practice sub-domains emerged: 1) acute pain and perioperative management; 2) OAT continuation; 3) opioid withdrawal management; and 4) OAT initiation.

4.7.1a. Acute pain and perioperative treatment: Guidance documents. Three hospital guidance documents (Hospitals A, C, and K) explicitly addressed OUD-related acute pain and perioperative management approaches. The documents, all hospital guidelines, recommended the same general practice-OAT continuation for all patients who had previously established OAT in the outpatient setting. Hospital K differed from the others by recommending that pregnant patients on buprenorphine, in need of additional opioid agonists, be discontinued from buprenorphine when additional opioids were required for pain control. Hospital $\mathrm{C}$ and $\mathrm{K}$ included recommendations for buprenorphine and methadone, and Hospital A recommendations were for buprenorphine only. All the guidelines recommended the use of non-opioid adjuvants to manage pain in the pre-operative and post-operative period.

All three guidelines recommended the use of opioid agonists for breakthrough and severe pain. Recommendations (Hospital C and K) explicitly noted that opioid dosing would likely be higher due to tolerance. All three guidelines suggested alternative OAT dosing approaches to manage pain. Two guidelines (Hospital A and Hospital K) recommended increasing the buprenorphine dose and all three guidelines 
recommended increasing OAT dosing frequency. All three guidelines included action related to discharge planning to ensure continuity of OUD treatment. Table 4.5 (after Section 4.7.1b) summarizes and compares the care policy domains (i.e., continuation of OAT, the use of non-opioid adjuvants, the use of opioids, alternative OAT approaches, and discharge planning).

4.7.1b. Acute pain and perioperative treatment: Interviews. Informants that did not have or provide analyzable documents described routine practice and care considerations for acute pain management and perioperative management for patients with OUD. There was a consensus among informants that daily OAT should be continued during hospitalization and that the buprenorphine dose could be increased or split to manage pain: "With methadone typically, we will just continue it and use a full agonist on top of it.... I think in general we find that just continuing it [buprenorphine] is the best policy. That is generally what we recommend" (D1) and:

We do not have an official procedure or guideline but our consult service recommendations would vary if the patient was on methadone or buprenorphine. For methadone we keep it on and have them add pain medication. For buprenorphine we may keep it on and split it, or slightly increase the dose, or hold it and just give more pain meds and then help advise them when to restart it. (I1)

At some institutions this practice was described as occurring on a case-by-case basis: 
We treat every case individually. The degree of the pain the person is in, how long we expect them to be in pain, what their goals are. I had a consult with a woman with septic arthritis of her hip and severe pain. Her long-term goal was to be on methadone maintenance which she had been on before but was not currently on. We treated her pain with methadone in divided doses and she needed six weeks of intravenous antibiotics. So, she went to a nursing home on methadone for her pain and her addiction treatment. It really depends. I had another patient with more minor pain...so we gave him buprenorphine he was happy with that and he followed up with me in clinic. It depends on the patient. (D1)

Informants commented that managing this clinical scenario, continuing OAT through

surgery or pain, frequently required a phone consultation or in-person consultation with

an addiction medicine expert or the AMC service:

They tend to consult us pretty frequently for that...I think there is...from the perspective of addiction medicine we tend to recommend continuing their basal opioid requirement and treating acute pain on top of that. I do not think that is universally recognized way to do things and so often times they will appreciate getting our input on that issue. (J1)

Challenges to effective pain management for patients on OAT were related to either the limited evidence base or the limited knowledge base about OAT among hospital staff:

I think the biggest barrier is we do not have great evidence to say this is the best way to do it. If there were great studies that we could point out that shows definitively that this the right thing to do for this patient. We rely more on a combination of clinical experience, observational studies, general knowledge of pharmacology...It is hard to convince somebody this is the right way to do it when I do not have evidence to back me up...I think that creates a lot of confusion in this area in treating pain in people on buprenorphine. (D1) 
Table 4.5. Guidance Document Recommendations: Pain Management ${ }^{\mathrm{a}}$

\begin{tabular}{|c|c|c|c|c|}
\hline Hospital & $\begin{array}{c}\text { Use of } \\
\text { Non-Opioid Adjuvants }\end{array}$ & $\begin{array}{l}\text { Use of } \\
\text { Opioids }\end{array}$ & $\begin{array}{c}\text { Alternative OAT } \\
\text { Approaches }\end{array}$ & $\begin{array}{l}\text { Discharge } \\
\text { Planning }\end{array}$ \\
\hline A & $\begin{array}{l}\text { Pre-Operative } \\
\text { Acetaminophen; NSAIDs; gabapentin } \\
\text { Intra-Operative } \\
\text { Regional anesthesia; continuous infusion } \\
\text { catheter; ketamine, lidocaine, or } \\
\text { dexmedetomidine infusion } \\
\text { Post-Operative } \\
\text { Ketamine, lidocaine, or dexmedetomidine } \\
\text { infusion }\end{array}$ & $\begin{array}{l}\text { Post-Operative } \\
\text { Intravenous hydromorphone or } \\
\text { fentanyl patient-controlled } \\
\text { analgesia }\end{array}$ & $\begin{array}{l}\text { Post-Operative } \\
\text { Increase } \\
\text { buprenorphine } \\
\text { dose or divide } \\
\text { daily dose to } \\
\text { every } 6 \text { to } 8 \text { - } \\
\text { hours }\end{array}$ & $\begin{array}{l}\text { Continue inpatient pain } \\
\text { management and coordinate } \\
\text { follow-up with outpatient } \\
\text { buprenorphine provider }\end{array}$ \\
\hline C & $\begin{array}{l}\text { Pre-Operative } \\
\text { Ibuprofen, acetaminophen, or topical } \\
\text { analgesics; gabapentin; tricyclic } \\
\text { antidepressants; serotonin and norepinephrine } \\
\text { reuptake inhibitors; tizanidine, baclofen, or } \\
\text { cyclobenzaprine; sedating serotonin reuptake } \\
\text { inhibitors; ketamine or dexmedetomidine; } \\
\text { neuraxial, regional, and local anesthesia } \\
\text { Post-Operative } \\
\text { Use multiple modalities }\end{array}$ & $\begin{array}{l}\text { Pre-Operative } \\
\text { Increase opioids and consult the } \\
\text { pain service. If opioids are needed } \\
\text { will likely need to provide higher } \\
\text { dose and monitor closely } \\
\text { Post-Operative } \\
\text { Manage similar to routine } \\
\text { postoperative care but may need } \\
\text { higher opioid dosing }\end{array}$ & $\begin{array}{l}\text { Pre-Operative } \\
\text { Split } \\
\text { buprenorphine } \\
\text { dose or dose } \\
\text { methadone } \\
\text { three times a } \\
\text { day }\end{array}$ & $\begin{array}{l}\text { Coordinate care. Specific } \\
\text { accommodations for skilled } \\
\text { nursing facility, outpatient living } \\
\text { situations, and coordination with } \\
\text { OTP or outpatient buprenorphine } \\
\text { provider. Including writing a } \\
\text { discharge bridge script for } \\
\text { buprenorphine }\end{array}$ \\
\hline K & $\begin{array}{l}\text { Pre-Operative } \\
\text { Multimodal management with non-opioids } \\
\text { (NSAIDs, acetaminophen, epidural/spinal } \\
\text { analgesics, and nerve blocks) }\end{array}$ & $\begin{array}{l}\text { Post-Operative } \\
\text { If opioids are required, will likely } \\
\text { need higher doses and they should } \\
\text { use patient-controlled analgesia } \\
\text { and discontinue oral opioids }\end{array}$ & $\begin{array}{l}\text { Post-Operative } \\
\text { Split } \\
\text { buprenorphine } \\
\text { dose or increase } \\
\text { buprenorphine } \\
\text { dose }\end{array}$ & $\begin{array}{l}\text { The patient should receive a } \\
\text { letter for OTP. The discharge case } \\
\text { manager and patient may need to } \\
\text { arrange for home methadone } \\
\text { doses with OTP if they cannot } \\
\text { receive on their day of discharge }\end{array}$ \\
\hline
\end{tabular}


4.7.1c. OAT continuation: Guidance documents. Informants from four hospitals

(Hospitals C, D, I, and K) provided OAT continuation guidance documents. Hospitals C, I, and $\mathrm{K}$ provided recommendations for buprenorphine and methadone, and Hospital D provided methadone recommendations. The consensus of the four guidance documents was that OAT should be continued and providers should consult with either the AMC service or addiction experts as needed.

Policies typically required dose verification for patients taking buprenorphine. All three guidance documents described steps for completing this task, including contacting the pharmacy (Hospital C), reviewing the electronic health record (Hospitals C and $\mathrm{K}$ ), checking the prescription drug monitoring program (PDMP) (Hospitals C, I, and K), contacting the outpatient clinic (Hospital I), and contacting the outpatient provider (Hospital K). Two of the hospitals provided recommendations for OAT continuation (Hospitals $\mathrm{C}$ and $\mathrm{K}$ ) and two of the documents addressed pain management (Hospitals $\mathrm{C}$ and $\mathrm{K}$ ). The other care delivery recommendation domains inconsistently articulated among the guidelines were approaches to missed doses, linkage to care, and legal reminders. See Table 4.6 (following Section 4.7.1d) for a summary of these findings.

All four methadone continuation guidelines recommended dose verification by contacting the patient's OTP. One guideline recommended use of the prescription bottle to verify dose (Hospital I). Three of the documents addressed pain management (Hospitals C, D, and K) in particular to provide additional opioids for pain as needed. Three of documents included instructions on methadone formulation (i.e., liquid, tablet, 
injectable) (Hospitals D, I, and K), and two of those hospitals (Hospitals D and K) described the OAT conversion to intramuscular injection. Three hospitals described linkage to care processes (Hospitals D, I, and K) including the operational limits of OTP and how to ensure communication with the OTP on the last dose received. Three hospitals provided legal reminders (Hospitals C, I and K) about methadone (e.g., do not write an outpatient prescription for methadone). The other care delivery recommendation domains not consistently described across the guidelines were how to address missed doses and naloxone orders. See Table 4.7 (following Section 4.7.1d) for a summary of these findings.

4.7.1d. OAT continuation: Interviews. Informants that did not have or provide analyzable documents described OAT continuation practice as similar to the provided and reviewed hospital policies. The most common approach was OAT continuation without restrictions upon dose verification from the outside provider: "We continue it absolutely it is an ethical and legal obligation" ( $\mathrm{H} 1)$ and "We get confirmation from their current providers and as long as we get confirmation about their dose and that the provider plans to continue to follow them and then we continue it [for both types of OAT]" (L1). Dose verification was completed by different members of the team (e.g., nursing or pharmacy) and involved using different verification sources depending on OAT-for buprenorphine (PDMP) or for methadone (OTP or the state substance abuse authority). Informants described methadone dose verification as more challenging than 
buprenorphine verification, because methadone is not in the state PDMP; however, one informant cautioned against trusting the PDMP for buprenorphine dose verification.

Key informants described OAT continuation, specifically for buprenorphine, as being dependent on the hospital formulary. One hospital switched patients from Suboxone ${ }^{\circledR}$ (the combination product) to Subutex ${ }^{\circledR}$ (the mono product) because of the formulary limitations and another hospital did the opposite, switching patients arriving on Subutex ${ }^{\circledast}$ to Suboxone ${ }^{\circledast}$. Some key informants were unsure about general hospital practice for this clinical scenario, stating that they did not "think there are policies right now [stating] that we have to do certain medication protocols. As far as I know it is physician discretion" (M1). Further, an informant noted that fear among the providers drove discontinuation of outside methadone because providers did not understand federal treatment regulations:

The majority of the time [patients on methadone are discontinued while hospitalized]. Unless they have a reason, pain. I have had these discussions with docs. They are nervous about using it for non-pain services and they are worried that they are doing something illegal. (M1)

Another key informant described how anyone admitted to the hospital on OAT triggers a consultation from the AMC service:

There is almost a sort of automatic trigger finger type response for most of the hospitals to call addiction medicine for anything. If somebody comes in on buprenorphine or methadone it is almost guaranteed they will consult us. If they have anything in their urine at all they are going to consult us. (L1) 
Table 4.6. Guidance Document Recommendations: Buprenorphine Continuation ${ }^{\mathrm{a}}$

\begin{tabular}{|c|c|c|c|c|c|c|}
\hline Hospital & Formulation & $\begin{array}{c}\text { Dose } \\
\text { Verification }\end{array}$ & $\begin{array}{c}\text { Missed } \\
\text { Dose }\end{array}$ & $\begin{array}{c}\text { Pain } \\
\text { Management }\end{array}$ & $\begin{array}{l}\text { Linkage } \\
\text { to Care }\end{array}$ & $\begin{array}{c}\text { Legal } \\
\text { Reminder }\end{array}$ \\
\hline C & $\begin{array}{l}\text {-Buprenorphine } \\
\text { /naloxone (non- } \\
\text { pregnant) } \\
\text {-Buprenorphine } \\
\text { (pregnant) }\end{array}$ & $\begin{array}{l}\text { - Contact pharmacy } \\
\text { - Review electronic } \\
\text { health record } \\
\text { - Check PDMP }\end{array}$ & $\begin{array}{l}\text {-If a patient has not used } \\
\text { opioids in the interim, } \\
\text { provider may order full } \\
\text { outpatient dose } \\
\text { - If a patient has used } \\
\text { opioids in the interim use } \\
\text { clinical judgment }\end{array}$ & $\begin{array}{l}\text { Continue dose } \\
\text { during acute } \\
\text { events }\end{array}$ & Not described & Not described \\
\hline I & Not described & $\begin{array}{l}\text {-Contact clinic } \\
\text {-Check PDMP }\end{array}$ & Not described & Not described & Not described & Not described \\
\hline K & $\begin{array}{l}\text { Use the } \\
\text { prescribed } \\
\text { outside } \\
\text { formulation }\end{array}$ & $\begin{array}{l}\text { Institutional Clinic } \\
\text { - Check electronic health } \\
\text { record } \\
\text { Outside Clinic } \\
\text { - Contact provider } \\
\text { - Check PDMP }\end{array}$ & Not described & $\begin{array}{l}\text { If dose does not } \\
\text { provide adequate } \\
\text { analgesia, use } \\
\text { non-opioids and } \\
\text { opioids and } \\
\text { follow pain } \\
\text { management } \\
\text { protocol }\end{array}$ & $\begin{array}{l}\text { On day of } \\
\text { discharge notify } \\
\text { outpatient } \\
\text { provider of } \\
\text { plans }\end{array}$ & $\begin{array}{l}\text { Do not write an } \\
\text { outpatient } \\
\text { prescription } \\
\text { unless you } \\
\text { have an X- } \\
\text { waiver and the } \\
\text { outside } \\
\text { provider asks } \\
\text { you to }\end{array}$ \\
\hline
\end{tabular}


Table 4.7. Guidance Document Recommendations: Methadone Continuation ${ }^{\mathrm{a}}$

\begin{tabular}{|c|c|c|c|c|c|c|c|}
\hline Hospital & $\begin{array}{c}\text { Dose } \\
\text { Verification }\end{array}$ & $\begin{array}{l}\text { Missed } \\
\text { Dose }\end{array}$ & $\begin{array}{c}\text { Pain } \\
\text { Management }\end{array}$ & $\begin{array}{l}\text { Form- } \\
\text { ulation }\end{array}$ & $\begin{array}{c}\text { Naloxone } \\
\text { Order }\end{array}$ & $\begin{array}{l}\text { Linkage } \\
\text { to Care }\end{array}$ & $\begin{array}{c}\text { Legal } \\
\text { Reminder }\end{array}$ \\
\hline C & Contact OTP & $\begin{array}{l}\text { - Discuss with OTP } \\
\text { provider } \\
\text { - If OTP cannot be reached } \\
\text { give full dose if } 1-2 \text { days } \\
\text { are missed, half dose if } 3 \text { - } \\
4 \text { days are missed, and } \\
\text { restart if } \geq 5 \text { days are } \\
\text { missed }\end{array}$ & $\begin{array}{l}\text { Short-acting } \\
\text { opioids or split } \\
\text { dosing }\end{array}$ & $\begin{array}{l}\text { Not } \\
\text { described }\end{array}$ & Yes & Not described & $\begin{array}{l}\text { Legal to } \\
\text { order if } \\
\text { patient is } \\
\text { admitted } \\
\text { primarily for } \\
\text { other } \\
\text { medical } \\
\text { reason }\end{array}$ \\
\hline D & Contact OTP & Not described & $\begin{array}{l}\text { Do not } \\
\text { withhold with } \\
\text { opioids and do } \\
\text { not subsite as } \\
\text { analgesic }\end{array}$ & $\begin{array}{l}\text { If NPO, use } \\
\text { IM } \\
\text { injection }\end{array}$ & $\begin{array}{l}\text { Not } \\
\text { described }\end{array}$ & $\begin{array}{l}\text { - Direct questions about } \\
\text { addiction treatment to OTP } \\
\text { - Prior to discharge notify } \\
\text { OTP of last dose amount } \\
\text { and date/time } \\
\text { - Write note for patient to } \\
\text { take to OTP with dose } \\
\text { information }\end{array}$ & $\begin{array}{l}\text { Not } \\
\text { described }\end{array}$ \\
\hline I & $\begin{array}{l}\text { Contact OTP } \\
\text { and/or use } \\
\text { prescription } \\
\text { bottle }\end{array}$ & $\begin{array}{l}\text { Do not attempt to make } \\
\text { up missed dose } \\
\text { - If }>3 \text { days of dosing } \\
\text { missed decrease verified } \\
\text { daily dose by } 10 \% \text { or } 10 \\
\text { mg for each day missed }\end{array}$ & Not described & $\begin{array}{l}\text { Continue } \\
\text { dose in } \\
\text { any form } \\
\text { (pill or } \\
\text { liquid) }\end{array}$ & $\begin{array}{l}\text { Not } \\
\text { described }\end{array}$ & $\begin{array}{l}\text { - OTPs are closed on holidays } \\
\text { and Sundays. } \\
\text { - OTP should be notified } \\
\text { when admitted and should } \\
\text { receive discharge summary }\end{array}$ & $\begin{array}{l}\text { Outpatient } \\
\text { pharmacies } \\
\text { cannot } \\
\text { dispense }\end{array}$ \\
\hline K & Contact OTP & Not described & $\begin{array}{l}\text { OAT does not } \\
\text { provide } \\
\text { analgesia, use } \\
\text { non-opioids } \\
\text { and opioids for } \\
\text { pain }\end{array}$ & $\begin{array}{l}\text { Continue } \\
\text { liquid } \\
\text { dose, if } \\
\text { NPO use } \\
\text { IM } \\
\text { injection }\end{array}$ & Yes & $\begin{array}{l}\text { At discharge provide letter } \\
\text { for OTP with admission dates } \\
\text { and date/amount of last } \\
\text { dose, names and dose of } \\
\text { other opioids given }\end{array}$ & $\begin{array}{l}\text { Do not write } \\
\text { outpatient } \\
\text { prescription }\end{array}$ \\
\hline
\end{tabular}




\subsection{1e. Opioid withdrawal management: Guidance documents. Withdrawal}

management policies were received from six hospitals (Hospitals $D, F, H, I, J$, and N). Similarities and differences among the protocols were observed among the recommendations for first-line therapy, the protocol type, the protocol initiation threshold, and the 24-hour maximum dose. Four of the six protocols specified the firstline medication for withdrawal management as one or both types of OAT. One hospital protocol was restricted to non-OAT pharmacotherapy symptom management (i.e., clonidine), and one hospital used a combination non-OAT pharmacotherapy (i.e., clonidine) and intramuscular buprenorphine injections. All of the protocols were framed as tapering protocols, and one protocol provided the option for OAT initiation (Hospital I). Each protocol had a different threshold for starting their protocol, as well as different total maximum doses for the first 24-hours. See Table 4.8 (following section 4.7.1f) for a summary of these findings.

4.7.1f. Interviews: Opioid withdrawal management. Informants that did not have or provide analyzable documents described a variety of practice approaches, which included the use of OAT to manage withdrawal, the use of non-opioid adjuvants for symptom management (i.e., clonidine), and the use of tramadol for a rapid taper. When queried about the distinction among induction versus withdrawal versus tapering, some informants emphasized that their approach was focused on "maintenance therapy" (A1). Other informants shared that there was variation across their respective hospital or health system for managing opioid withdrawal. For example, one informant stated 
that those admitted to the hospital detoxification unit received a tapering protocol that was for 72 hours and patients who were admitted to the main part of the hospital received a 72-hour to 120 -hour tapering protocol. Another informant described little to no management for opioid withdrawal across their institution.

Table 4.8. Guidance Document Recommendations: Withdrawal Management

\begin{tabular}{|c|c|c|c|c|}
\hline Hospital & $\begin{array}{c}\text { First-Line } \\
\text { Therapy }\end{array}$ & $\begin{array}{l}\text { Protocol } \\
\text { Initiation }\end{array}$ & Starting Dose & $\begin{array}{c}\text { 24-Hour Maximum } \\
\text { Dose }\end{array}$ \\
\hline $\mathrm{D}$ & $\begin{array}{l}\text { Sublingual } \\
\text { BUP-NX }\end{array}$ & COWS $\geq 8$ & $4 \mathrm{mg}$ & Not described \\
\hline $\mathrm{F}$ & $\begin{array}{l}\text { Sublingual } \\
\text { BUP-NX }\end{array}$ & COWS $\geq 13$ & $\begin{array}{l}\text { Sublingual BUP-NX } \\
4 \mathrm{mg} / 1 \mathrm{mg} \\
\text { Oral or IV Methadone } \\
5 \mathrm{mg} \text { (COWS } 13 \text { to } 24 \text { ) and } \\
10 \mathrm{mg} \text { (COWS } 25 \text { to } 36 \text { ) }\end{array}$ & $\begin{array}{l}\text { Sublingual BUP-NX } \\
8 \mathrm{mg} \\
\text { Oral or IV Methadone } \\
40 \mathrm{mg} \text { oral or } 20 \mathrm{mg} \mathrm{IV}\end{array}$ \\
\hline $\mathrm{H}$ & $\begin{array}{l}\text { Sublingual } \\
\text { BUP-NX or } \\
\text { Methadone }\end{array}$ & COWS $\geq 12$ & $\begin{array}{l}\text { Sublingual BUP-NX } \\
4 \mathrm{mg} / 1 \mathrm{mg} \\
\text { Oral Methadone } \\
15 \mathrm{mg}\end{array}$ & $\begin{array}{l}\text { Sublingual BUP-NX } \\
8 \text { mg (10 mg severe) } \\
\text { Oral Methadone } \\
30 \mathrm{mg} \text { ( } 35 \mathrm{mg} \text { severe) }\end{array}$ \\
\hline I & Methadone & $\begin{array}{l}\text { Signs of } \\
\text { withdrawal } \\
\text { or opioid } \\
\text { craving }\end{array}$ & $\begin{array}{l}\text { Oral Methadone } \\
5 \text { to } 10 \mathrm{mg}\end{array}$ & $\begin{array}{l}\text { Oral Methadone } \\
40 \mathrm{mg}\end{array}$ \\
\hline J & Clonidine & $\begin{array}{l}\text { Not } \\
\text { described }\end{array}$ & $\begin{array}{l}\text { Clonidine Standard } \\
0.2 \mathrm{mg} \\
\text { Clonidine Reduced } \\
0.1 \mathrm{mg} \\
\text { Clonidine Lowest } \\
0.05 \mathrm{mg}\end{array}$ & $\begin{array}{l}\text { Dependent on regimen } \\
\text { selected }\end{array}$ \\
\hline $\mathrm{N}$ & $\begin{array}{l}\text { IM } \\
\text { Buprenorphine } \\
\text { and Oral } \\
\text { Clonidine }\end{array}$ & COWS $\geq 5$ & $\begin{array}{l}\text { Clonidine } \\
0.1 \mathrm{mg} \\
\text { IM Buprenorphine } \\
0.3 \mathrm{mg}\end{array}$ & $\begin{array}{l}\text { Clonidine } \\
1.2 \mathrm{mg} \text { (max } 0.4 \mathrm{mg} \\
\text { dose every } 8 \text { hours) } \\
\text { IM Buprenorphine } \\
1.5 \mathrm{mg}(0.3 \mathrm{mg} \times 3 \text {, plus } \\
0.6 \mathrm{mg} \text { dose) }\end{array}$ \\
\hline
\end{tabular}

Table Notes. BUP-NX = buprenorphine/naloxone; COWS = Clinical Opiated Withdrawal Scale is a tool for assessing the severity of withdrawal symptoms using an 11-item assessment administered in either the outpatient or inpatient setting (Wesson \& Ling, 2003); IV = intravenous; IM = intramuscular. 
4.7.1g. OAT initiation: Guidance documents. Two hospitals (Hospital C and K) provided OAT initiation hospital guidance documents in the form of guidelines, protocols, and order sets. There were few common recommendations across the two guidelines. For buprenorphine initiation, Hospital C and Hospital K had different protocol initiation thresholds with differences in Clinical Opiate Withdrawal Scale (COWS) score thresholds to initiate OAT and allowable maximum daily doses (16 mg versus $12 \mathrm{mg}$ ). The first day dosing protocols were also different, in timing, dosing, and cOWS score threshold.

Hospitals $\mathrm{C}$ and $\mathrm{K}$ also had different methadone protocols including the threshold for protocol initiation, the maximum daily dose during hospitalization (60 mg versus $40 \mathrm{mg}$ ), and the first day dosing protocol. The common recommendation was the maximum total daily dose of methadone on day 1 of the protocol, $40 \mathrm{mg}$. See Table 4.9 (following section 4.7.1h) for a summary of these findings.

4.7.1h. Interviews: OAT initiation. Informants that did not have or provide analyzable documents disclosed a range of practice behaviors related to OAT initiation. At some institutions the delivery environment focused on OAT initiation versus withdrawal management:

Methadone or buprenorphine can be initiated during hospitalization, in consultation with an addiction medicine specialist, if the patient will be detoxed off during hospitalization or there is a clear documented plan for continuation of OAT in the outpatient setting. (01)

In contrast, some hospital environments were described as underdeveloped in this practice domain: "I am unaware of anyone doing that...And I am unaware of any 
hospital policies [on this topic]" (M1). One informant described extreme frustration that

OAT initiation was not happening for hospitalized patients:

No! No! No! That pisses me off!!... So what is happening is that people are getting withdrawal management, tapered, and then sent to a clinic, where I reinduce them...It is a source of frustration. (F1)

Informants provided insight into some of the barriers to hospital OAT initiation,

which included: 1) OTPs requiring QTc interval testing; 2) internalized stigma of patients;

and 3) providers not trained in administering OAT. OAT initiation in many hospitals was

described as primarily the responsibility of the consult service:

What I would say around that is that it is quite rare [for physicians to do buprenorphine inductions]. I think there are some family medicine providers that do inductions in the outpatient setting and that will do them without our consult service. Other than that, I have not seen it. (A2)

Occasionally [physicians who are not addiction medicine specialist will induct patients on OAT and] there will be some miscommunications and people get started on methadone and all of a sudden, they are being discharged and how are they going to get connected with the methadone clinic. Sometimes surgical teams will start buprenorphine for patients, and not involve us, which is fine, but I think it just leaves some potential for gaps in their treatment, like what if they don't have that Suboxone ${ }^{\circledR}$ at home anymore because they missed their appointment because they were in the hospital, should they be back on the same dose or not? So just stuff like that will occasionally get missed. (I1)

Some informants described a preference for providing different types of OAT in the hospital. One informant emphasized their preference for starting buprenorphine over methadone in the hospital context: "I really prefer buprenorphine" (P1). Other informants discussed trying to use different non-OAT opioid formulations, such as BuTrans ${ }^{\circledast}$ patches, but were limited by the hospital formulary (E1). 
Table 4.9. Guidance Document Recommendations: OAT Initiation

\begin{tabular}{|c|c|c|c|c|}
\hline Hospital & $\begin{array}{l}\text { Protocol Initiation } \\
\text { coWS Score }^{1}\end{array}$ & $\begin{array}{l}\text { Max Total } \\
\text { Dose Day } 1\end{array}$ & $\begin{array}{c}\text { Max Daily Dose } \\
\text { During Admission }\end{array}$ & $\begin{array}{c}\text { First Day } \\
\text { Dose Protocol }\end{array}$ \\
\hline \multicolumn{5}{|c|}{ Buprenorphine } \\
\hline $\mathrm{C}$ & $\begin{array}{l}\text { Dependent on patient } \\
\text { category } \\
\text { Abstinent } \\
\text { No opioid use in the } \\
\text { prior } 5 \text { days: COWS }=0 \\
\text { All Others } \\
\text { COWS } \geq 8\end{array}$ & $\begin{array}{l}16 \mathrm{mg} \text { for } \\
\text { both types } \\
\text { of patients }\end{array}$ & $\begin{array}{l}24 \mathrm{mg} \text { for both } \\
\text { types of patients }\end{array}$ & $\begin{array}{l}\text { Abstinent } \\
\text { - Dose } 1 \text { is } 2 \mathrm{mg} \\
\text { - COWS score to be reassessed after } 2 \text { hours } \\
\text { - If cravings persist add another } 2 \mathrm{mg} \text {. This may be repeated on day } 1 \\
\text { until max is reached } \\
\text { All Others } \\
\text { - For patients with coWs }<8 \text { or no objective withdrawal, the COWS } \\
\text { should be administered every } 2 \text { hours until cOWS is } \geq 8 \\
\text { - When COWS } \geq 8 \text {, administer } 4 \mathrm{mg} \text { and assess COWS in } 1 \text { hour. If } \\
\text { COWS is }<8 \text { at that time, reassess every } 6 \text { hours } \\
\text { - Provider may give } 4 \mathrm{mg} \text { if COWS } \geq 8 \text { at any point }\end{array}$ \\
\hline $\mathrm{K}$ & $\begin{array}{l}- \text { COWS } \geq 5 \text { OR; } \\
\text { - The presence of } \\
\text { signs/symptoms of } \\
\text { withdrawal }\end{array}$ & $12 \mathrm{mg}$ & $16 \mathrm{mg}$ & $\begin{array}{l}\text { - Regardless of the historic amount of opioid use start with } \\
\text { buprenorphine/naloxone sublingual } 4 / 1 \mathrm{mg} \text { for dose } 1 \\
\text { - Reevaluate the patient every } 2 \text { to } 3 \text { hours } \\
\text { - Give additional dose increments of } 4 / 1 \mathrm{mg} \text { every } 2 \text { to } 3 \text { hours until } \\
\text { withdrawal abates (COWS }<5 \text { ) }\end{array}$ \\
\hline \multicolumn{5}{|r|}{ ( } \\
\hline C & Not described & $40 \mathrm{mg}$ & $60 \mathrm{mg}$ & The initial dose selection should start anywhere from 10 to $30 \mathrm{mg}$ \\
\hline K & $\begin{array}{l}\text { COWS } \geq 5 \text { or the } \\
\text { presence of } \\
\text { signs/symptoms of } \\
\text { withdrawal }\end{array}$ & $40 \mathrm{mg}$ & $40 \mathrm{mg}$ & $\begin{array}{l}\text { - Regardless of the historic amount of opioid use start with } \\
\text { methadone } 20 \mathrm{mg} \text { solution by mouth for dose } 1 \\
\text { - Reevaluate the patient every two to three hours } \\
\text { - Give additional doses increments of } 5 \text { to } 10 \mathrm{mg} \text { every } 2 \text { to } 3 \text { hours } \\
\text { until withdrawal abates (COWS }<5 \text { ) }\end{array}$ \\
\hline
\end{tabular}


4.7.2. Policy and practice domain 2: Security and behavior management. This

policy and practice domain addressed security and behavior management of patients with OUD and other SUDs. Informants from Hospitals A, B, J, and K provided hospital guidance documents in this policy domain. Specific protocols addressed the management of aberrant drug use, decision-making around peripherally inserted central catheter (PICC) lines, and patient safety and security assessment and interventions. The hospital guidance documents emphasized interprofessional collaboration to ensure patient safety; however, because the guidance documents varied in focus, most of the documents were difficult to compare, except for the aberrant drug use policies, which had enough shared properties for analysis.

4.7.2a. Guidance documents: Aberrant drug use. Aberrant drug use was defined as the use of drugs (illegal or prescribed) in a manner or route that was incongruent with appropriate or safe use. The policies on this topic from Hospitals B and J were similarly structured. Recommendations advised conducting a risk assessment for aberrant drug use; documentation of behavior and interventions protocols; interventions for medication administration; interventions for securing the environment; behavioral requirements for patients leaving the unit and for patient visitors; the use of urine drug screens; the use of patient sitters; and the involvement of campus safety. In Hospital B, the guideline was created because of a patient's poor outcome. Another issue related to aberrant drug use, which came up in the Hospital J guideline and in the interviews was limited access to sitters. The informant commented 
that there had been discussions about using video to monitor patients with drug use disorders:

This was a discussion [we had] quite recently because of increasing opioid related admissions. We have had more of this kind of issue [aberrant drug use] ... in the hospital or people being found with paraphernalia on them and the guidance at this point is essentially been assigning a sitter for those individuals. To observe. There has been a discussion to use video because sitters are a finite resource and there was getting to be issue with a little more frequency with some concern that there may not be enough sitter availability. (J1)

At present, according to the guideline, the use of video monitoring for this patient population is not allowed. The polices are summarized in Table 4.10 
Table 4.10. Guidance Document Recommendations: Aberrant Drug Use

\begin{tabular}{|c|c|c|c|c|c|c|c|c|c|}
\hline Hospital & $\begin{array}{c}\text { Risk } \\
\text { Assessment }\end{array}$ & Document & $\begin{array}{c}\text { Medication } \\
\text { Administration }\end{array}$ & UDS & $\begin{array}{l}\text { Visitor } \\
\text { Rules }\end{array}$ & $\begin{array}{l}\text { Environment } \\
\text { \& Equipment }\end{array}$ & $\begin{array}{c}\text { Limit } \\
\text { Off-Unit } \\
\text { Access }\end{array}$ & Sitter & $\begin{array}{l}\text { Security/Law } \\
\text { Enforcement }\end{array}$ \\
\hline $\mathrm{B}^{1}$ & $\begin{array}{l}\text { Physical } \\
\text { findings, } \\
\text { patient history, } \\
\text { patient } \\
\text { behaviors, and } \\
\text { laboratory } \\
\text { findings }\end{array}$ & $\begin{array}{l}\text { Document } \\
\text { ation of } \\
\text { behavior }^{a, b}\end{array}$ & $\begin{array}{l}\text { - Convert pills to } \\
\text { liquid }^{a} \\
\text { - Crush pills } \\
\text { - Change opioid } \\
\text { formulary } \\
\text { - Direct } \\
\text { observation } \\
\text { - Oral check } \\
\text { a }\end{array}$ & Yes $^{a}$ & $Y_{e s}^{b}$ & $\begin{array}{l}\text { - Remove sharps } \\
\text { container from } \\
\text { room } \\
\text { - Move patient } \\
\text { room close to } \\
\text { nursing station } \\
\text { - Leave door } \\
\text { open }^{\text {b }} \\
\text { - Application of } \\
\text { tamper } \\
\text { prevention } \\
\text { seals on all IV } \\
\text { ports }\end{array}$ & $Y_{e s}^{b}$ & $Y_{e s}^{b}$ & $\begin{array}{l}\text { Search room and } \\
\text { belongings }^{b}\end{array}$ \\
\hline $\mathrm{J}^{2}$ & $\begin{array}{l}\text { Moderate } \\
\text { concern, high } \\
\text { concern, or } \\
\text { confirmed use }\end{array}$ & $\begin{array}{l}\text { Objective } \\
\text { charting } \\
\text { indicating } \\
\text { evidence } \\
\text { of actions } \\
\text { taken and } \\
\text { the } \\
\text { patient's } \\
\text { response } \\
\text { to these } \\
\text { actions }\end{array}$ & $\begin{array}{l}\text { - Direct observation } \\
\text { - Check for } \\
\text { swallowing } \\
\text { - Crush medication } \\
\text { and put it in } \\
\text { pudding } \\
\text { - Limit IV access }\end{array}$ & Yes & Yes & $\begin{array}{l}\text { - Remove sharps } \\
\text { container from } \\
\text { room } \\
\text { - Do not leave } \\
\text { needles/syringe } \\
\text { s in the room } \\
\text { - Re-search the } \\
\text { room if there is } \\
\text { evidence of } \\
\text { "contraband" }\end{array}$ & Yes & Yes & $\begin{array}{l}\text { - Security should be } \\
\text { informed of any } \\
\text { substances or } \\
\text { paraphernalia found } \\
\text { - Security will } \\
\text { determine if police } \\
\text { involvement is } \\
\text { necessary } \\
\text { - Security and nursing } \\
\text { may search room } \\
\text { including patient } \\
\text { belongings }\end{array}$ \\
\hline
\end{tabular}


The other hospital guidance documents comprising this domain were on topics related to decision-making for patient discharge with PICC lines and patient safety and security assessments and interventions. Hospital A provided several hospital guidance documents related to PICC lines and aberrant drug use, including a PICC line policy and PICC assessment tool, an internal and externally facing behavior agreement policy, and a care misalignment document. An informant from Hospital A described the involvement of the hospital AMC service when behavioral issues arose, in particular for the misuse of drugs in the hospital setting:

[This is] part of the consult services task. We incorporate that into the behavioral agreement. It is one of the areas of culture change. Previously that had been a deal beaker for staying in the hospital. Because we are willing to engage with the patient... The truth is. If someone is on appropriate dosing of buprenorphine and methadone they are not going to want to use. They use in the hospital because they are in pain or on the wrong dose... We do not kick people out because they use. (A1)

The Hospital A PICC Policy and PICC Assessment Tool standardized PICC related discharge decision-making for high risk patients. The PICC Policy stated that PICC placement is a medical decision to be determined by a physician and informed by previous risk factors.

The Hospital A Safety Agreement Policy was an internally facing (for staff) and externally facing (for patients) policy. The internal component addressed how to identify a patient at risk for care misalignment, elopement, or harm to self or others; how to prepare and implement a safety plan; how to document the plan; how to handle conflict; and how to prepare a crisis plan. The component of the policy for patients 
communicated both behavioral expectations (e.g., no drug use) and care delivery expectations while in the hospital (e.g., understand the role of nursing).

The Hospital A Misalignment of Care Guideline was a tool designed to support interprofessional care plan meetings to facilitate care for patients at risk for misalignment in their care goals or the use of force. It outlined the steps to be taken to facilitate and implement a plan to keep at-risk patients safe.

Hospital K's Against Medical Advice (AMA) Policy provided a clear set of steps for action when patients leave the hospital AMA. Four steps were recommended when a patient says they are going to leave AMA: 1 ) discourage leaving and explain why it is important to stay; 2 ) inform the primary team; 3 ) if the patient still chooses to leave, staff ask the patient to sign the AMA form and document this conversation in the medical record; and 4) the event is documented in the patient record and filed in a reporting system. The guidance documents from Hospital $\mathrm{A}$ and Hospital K were not included in the comparison table because they were non-comparable, standalone protocols. A full summary of each set of recommendations is available by request from the primary researcher.

4.7.2b. Interviews: Security and behavior management. Similar to the findings observed in the hospital guidance documents, key informants who did not provide guidance documents from this policy domain described care scenarios and practice issues related to aberrant drug use and PICC line safety. 
4.7.2b.1. Aberrant drug use. Informants shared that it was the role and responsibility of addiction medicine and the interprofessional teams when there is ongoing aberrant drug use during hospitalization: "It certainly comes up. I get called about it sometimes. I get providers who are concerned about it sometimes and we will talk through it" (C1); "We get calls about people who smuggle in opioids and use them in the shower and have taken fentanyl or something that they have smuggled in" (M1); and "But this is another reason we sometimes we get consulted. Somebody has been hoarding medications and then crushing their oxy and injecting it through their port. Um friends bringing in their benzos from home" (F1).

Many informants, when asked about protocols and standard practice on aberrant drug use, indicated that there was limited hospital-wide understanding on what to do in these scenarios: "I am not personally aware of protocols around that. I do know that, I have seen certain situations of patients who have used in the hospital and they aren't automatically discharged or anything like that" (C1) and "No. No real policy. A lot of consternation" (N1). In contrast, other informants described a no-tolerance policy related to on-campus drug use that involved campus security:

Yeah it is obviously not allowed and security gets involved and you know it is pretty bad. I don't think they are looking to call the police but cognizant of the fact that this is illegal and can't go on...I think a lot of people will just AMA [leave against medical advice]. And leave. We have had a couple of situations where it is obvious they have called a drug dealer or something and they usually find themselves on the wrong side of security and they end up leaving. $(\mathrm{H} 1)$ 
We discourage it. I think there have been times were people caught using intravenous drugs in the hospital and you know, generally, security is sort of put outside their room and visitors are restricted and their belongings are generally searched. That is only if they are caught doing it. (L1)

4.7.2b.2. PICC lines. Informants discussed the role and responsibility of addiction medicine providers to support decision-making related to PICC line insertion or removal: "A common genre of consults is somebody with a PICC line" (F1). Hospital clinical staff would request that the $\mathrm{AMC}$ service conduct a risk assessment prior to discharging a patient with a PICC line and a history of intravenous drug use. General practice around PICC lines included efforts to discourage patients with intravenous drug use history from leaving the hospital with a PICC line: "No, we try not to let them leave the hospital with a PICC line if they have a history of intravenous use. Again, I think the nurses are kind of trained to be more vigilant in those cases" (L1).

Informants noted that addiction medicine experts were responsible for navigating the challenges of patient placement upon discharge because a patient had an active OUD, a need for OAT, and a PICC line:

We cannot discharge patients often times...[because] we can't get nurses to go into a home if there is a history of substance use... and they wouldn't be discharging a patient with a PICC line if there is a history [of use] ...I have a huge problem with nursing homes that will subtly discriminate against patients on methadone. This is a big issue. A big issue, right? I am just screaming for a lawsuit from somebody. (G1)

Any patient who needs a PICC line gets a PICC line. If patient requires long-term antibiotics (e.g., endocarditis, osteomyelitis) then patient is discharged to SNF for duration of antibiotics. Can be challenging if patient is on OAT-however we have worked with several area SNF who will accept patients who inject drugs with a PICC line on OAT. (01) 
Related to PICC line placement, one informant described the tension between

patient autonomy and hospital interventions:

There seems to be a profound concern among the PICC line insertion team that people are going to go and use their PICC line to get high, and I was like, so what? I mean it is their human right. It is a terrible idea and we can message them that is a really dangerous and really bad... my sense of their concern [the PICC line insertion team] was that they viewed the lines as their property and god-forbid the patient ran out and used with their IV in. I was just like patients have agency here and they are allowed to make horrible choices... we certainly put PICCs in OUD patients, but to be honest, I am sure they are mistreated, I know people judge them, I know people probably make remarks, probably not even behind their back right around them, oh he is going to do something with his PICC. Heck a PICC is safer than doing it yourself. (H1)

Informants also described other stigmatizing, and likely traumatizing behavioral management practices (e.g., no visitors allowed policies and searching patient's belongings):

I initiated a policy that when I am involved [in care] there is a no visitors policy for patients. And I say to them you cannot have visitors. I basically get their consent to put that in place. I explain to them you are sick and I do not want someone to bring something in to you it could put you in jeopardy and it can put the hospital staff in jeopardy. And many times, we have security sitting outside the door...but it is really not a problem people get it. (P1)

Behavioral issues were so common that one hospital created an interprofessional consult service to de-escalate patients with behavioral issues.

4.7.3. Interviews: Non-evidence-based care. Throughout the interviews, informants described a variety of non-evidence-based care practices for patients hospitalized with OUD. These care scenarios included patients receiving no treatment for their OUD, patients receiving incorrect or outdated care, and patients receiving 
uncommon care delivery modalities. The informants associated with these delivery scenarios have been deidentified.

4.7.3a. No treatment. One informant shared how the standard of care on the medical and surgical services at their hospital was to not provide any OUD-related care-even symptom management for withdrawal, referring to a patient with endocarditis secondary to intravenous drug use:

At the very least we will...treat their pain...But you know what we struggle with the most is these people who have endocarditis or are septic and have to have 6 weeks of antibiotics and they are not receiving anything for withdrawal...on some services maybe someone would write you something for pain... but that wouldn't necessarily be for withdrawal. They could give some clonidine. (deidentified)

4.7.3b. Incorrect or outdated care. Evidence supports the use of continued OAT in the outpatient setting to decrease return to substance use and death (Clark et.al, 2011). Despite this, informants described the provision of OUD-related services in their respective hospitals as focused on "detox" (i.e., withdrawing patients from opioids instead of initiating and continuing OAT upon discharge): "We have what we call a chemical opiate withdrawal protocol that we have been using for a number of years" (deidentified); "Generally they will use clonidine for detoxification purposes" (deidentified); and:

They come in, they get consulted by addiction medicine...[and] now, our policy/procedure is to utilize tramadol to detox along with as needed medications for all the symptoms, clonidine, muscle relaxer, something for nausea ease, Vistaril for anxiety things like that. We generally don't use buprenorphine or methadone anymore expect in pregnant patients we obviously start them on Subutex ${ }^{\circledast}$. (deidentified) 
4.7.3c. Uncommon care delivery modalities. Informants described unique withdrawal management protocols. The protocols do not appear to be supported by evidence, yet there is little third-party oversight in the hospital setting to ensure the use of validated treatment protocols. One of the protocols was a rapid 72-hour taper using tramadol, and the other was an injectable buprenorphine taper protocol. The informant rationalized the use of the rapid tramadol taper approach because of the 72-hour rule. She described that prior to the implementation of the tramadol protocol they had used Suboxone ${ }^{\circledR}$ on their detoxification unit, and the consult service. She said that once patients realized that they could only receive the medication for 72-hours they would leave AMA, she said it created a "revolving door, they would come and get a couple of days' worth [of Suboxone ${ }^{\circledR}$ ] and leave and come back to get more" (deidentified). She shared that using tramadol has minimized this effect.

4.7.4. Summary. The hospital guidance documents addressed similar clinical domains, but differences were observed within the policy sub-domains. Informants and guidance document recommendations related to acute pain management agreed; patients on OAT prior to admission should be continued on OAT during hospitalization. For withdrawal management, however, there was substantial variation among the guidance documents across almost all practice domains (i.e., first-line therapy, protocol initiation threshold, starting dose, and 24-hour maximum dose). This variation was also reflected in the key informant descriptions of care practices. For OAT initiation, again as with withdrawal management, the guidance documents were discordant across 
domains (i.e., protocol initiation threshold, maximum day 1 dose, maximum total daily dose, and first day dose protocol), and generally informants were unsure about the existence of common practices for OAT initiation in their institutions. The variation among the hospital guidance documents provide additional evidence of the novelty of this practice within hospitals, that there is limited standardization because few hospitals are doing it; thus, a national standard does not exist yet.

It was challenging to draw conclusions from the security and behavioral management sub-policy domains because the guidance documents varied in focus. They emphasized interprofessional collaboration to ensure patient safety. The two aberrant drug use policies had similar recommendations for assessments, documentation, interventions, patient and visitor requirements, the use of urine drug screening, and campus safety involvement. However, less consistency was observed in disclosed care practices related to aberrant drug use-most informants indicated limited hospital-wide understanding in addressing aberrant drug use. Less common, though highly problematic, was the existence of guidance documents and disclosures from informants during the interviews of the delivery of non-evidenced based care to persons with OUD. These non-evidence-based care scenarios included patients receiving no treatment for their OUD, patients receiving incorrect or outdated care, or receiving uncommon and untested protocols.

The findings from this section provide additional evidence to support the assertion that hospital policies and practice vary for the delivery of OAT and treatment 
of hospitalized patients with OUD, and that the existence of said policies and normative practices reflect specific supply-side attributes within and outside of the local hospital environment. The variation and care discrepancy existed within the context of 16 hospitals that are well-resourced for the delivery of addiction-related services in contrast with the average U.S. hospital without addiction medicine experts or addiction medicine training programs.

Finally, based on the evidence presented in the previous sections, triangulated with the observations from this section, AMC service existence is a conduit for OAT management policy creation and implementation. Seventy-eight percent of hospitals with established AMC services in this study had OAT management policies $(n=7)$ as compared with $0 \%$ of hospitals without an AMC service and the plans to start one. Further, $40 \%$ of hospitals with the plans to start an AMC service $(n=2)$ had OAT management guidance documents.

\section{Section 4.8. Linking Qualitative Findings to Quantitative Approach}

The analyses presented in this chapter provide the conceptual rationale and justification for continuing to explore care delivery practice, patterns, and organizational processes for patients with OUD in Aims 2 and 3. The findings from Chapter 4 identified care delivery patterns for the treatment of persons with OUD during hospitalization that appear to be suboptimal and not evidence-based. Informants described care variation within and between hospitals. The collection and analyses of hospital guidance documents reflected and confirmed this finding. These results provide rationale and 
justification for proceeding with Aims 2 and 3 to quantitatively explore, describe, and assess care delivery across the largest integrated health system in the U.S. - the Veterans Health Administration (VHA).

The results of this chapter illuminate gaps in fundamental knowledge as they relate to understanding the social and technical contributory elements that contributes to an addiction friendly hospital. Some of the findings from this section suggest that there are mutable social and technical attributes within and outside of a hospital that may enhance a hospital's ability to deliver evidence-based OUD services including: positive social structures (i.e., behaviors, cultural-cognitive, and normative), resource investments in addiction-related care (e.g., addiction medicine training programs or an AMC service), normative treatment practices (e.g., guidelines), and supportive internal stakeholders. Further, findings from this chapter illustrate the power of the local environment to enhance or foster the internal work of the hospital such as external stakeholders, a robust community-based referral network, external incentives through policy, regulations, financing, education, and knowledge. This emphasizes the theoretical underpinnings of this study that hospitals, like other organizations, do not operate in isolation but act as dynamic, malleable structures influenced by the broader social and professional contexts and systems. How these internal and external contributions are measured, defined, and quantified warrants future exploration. Moreover, the findings from this chapter inform the analytic approaches of Chapter 5, 
specifically how OAT was operationalized to include all OAT formulations of OAT and how hospitals were categorized as either high or low performers. 


\section{Chapter 5: Presentation and Analysis of Quantitative Data}

Aim 2 explores the variation in hospital use of opioid agonist therapy (OAT) and other non-OAT pharmacotherapies for hospitalized patients with OUD in the Veterans Health Administration (VHA) system. A description of the study cohort opens the chapter and reviews patient and facility characteristics, and pharmacotherapy delivery patterns. The Aim 2 research sub-questions analyze care delivery patterns and examine OAT administration variability among hospitalized veterans. Aim 3 analyses expand upon the findings of Aim 2 to further understand the multilevel drivers of hospital OAT variation in the VHA system. Results are organized into seven sections:

1. Section 5.1 describes the facilities, patient demographics, admission characteristics, patient health characteristics (present on admission and during admission), and pharmacotherapy delivery patterns (pre, during, and post-admission) of the study cohort.

2. Section 5.2 (research sub-questions $2 a, 2 b$, and $2 c$ ) examines the differences in the characteristics and outcomes of patients who do and do not receive OAT when hospitalized in the VHA.

3. Section 5.3 (research sub-questions $2 d, 2 e, 2 f$ ) explores the differences in the characteristics and outcomes for patients who received OAT prior to admission and were subsequently continued or discontinued.

4. Section 5.4 reviews the distribution and frequency of hospital OAT delivery within the study cohort. First, the distribution and frequency of hospital OAT 
and non-OAT delivery is broadly summarized (research sub-questions $2 \mathrm{~g}$ ).

Second, the associations between hospital performance quartile and specific OAT care delivery scenarios (research sub-questions $2 \mathrm{~h}$ ) are assessed. Third, hospital attributes associated with hospital OAT delivery (research subquestions 2i) are summarized. Fourth, differences in non-OAT pharmacotherapy delivery associated with hospital OAT delivery quartile performance (research sub-questions $2 \mathrm{j}$ ) are assessed. Finally, associations between health and health care utilization outcomes with hospital OAT delivery rank or quartile (research sub-questions $2 k$ ) are evaluated.

5. Section 5.5 summarizes the findings from Aim 2 that frame the Aim 3 analyses.

6. Section 5.6 explores the multilevel contributions of patients and hospital level attributes that are associated with the delivery of OAT during admission and the delivery of OAT in the 30 days post-admission.

7. Section 5.7 summarizes the findings of this chapter.

\section{Section 5.1. Patient, Admission, and Hospital Descriptive Statistics}

This section explores the facility, patient, and admission characteristics for the final study cohort-12,407 unique index hospitalizations from 109 VHA hospitals in the continental U.S. during the 2017 fiscal year.

5.1.1. Hospital characteristics. Most hospitals were in either the South $(n=43$; $39 \%$ ) or the Midwest ( $n=25 ; 23 \%$ ). Many facilities were large hospitals with more than 
100 beds ( $n=45 ; 41 \%$ ) or medium sized hospitals with 50 to 99 beds ( $n=36 ; 33 \%$ ).

Cases occurred most commonly in large hospitals ( $n=7,234 ; 58 \%)$ and in the South (39\%; $n=4,867)$. The number of patients with OUD admitted per hospital ranged from 26 to 430 with a median of $98(I Q R=97)$ (see Table 5.1).

Table 5.1. VHA Hospital Characteristics

\begin{tabular}{lcc}
\hline \multicolumn{1}{c}{ Variable } & $\begin{array}{c}\text { Number of Facilities } \\
(\mathrm{n}=109)\end{array}$ & $\begin{array}{c}\text { Patient Frequency } \\
(\mathrm{n}=12,407)\end{array}$ \\
\hline Admissions (count) & Median, 98; Mean, 114.0 & Range 26 - 430; IQR 97 \\
\hline Number of Beds & & \\
Small: 1 to 49 beds & 28 & $12.5 \%(1551)$ \\
Medium: 50 to 99 beds & 36 & $29.2 \%(3622)$ \\
Large: 100+ beds & 45 & $58.3 \%(7234)$ \\
\hline Census Region & 25 & $22.1 \%(2742)$ \\
Midwest & 18 & $16.4 \%(2035)$ \\
Northeast & 43 & $39.2 \%(4867)$ \\
South & 23 & $22.3 \%(2763)$ \\
West & &
\end{tabular}

5.1.2. Patient characteristics. Patient characteristics across the study cohort were similar. Most patients were male $(n=11,543 ; 93 \%)$, white $(n=8,880 ; 72 \%)$, and non-Hispanic or Latino ( $n=11,476 ; 93 \%)$. The median patient age was 61 years (range 21 to 90) (see Table 5.2). 
Table 5.2. VHA Patient Characteristics

\begin{tabular}{ll}
\hline \multicolumn{1}{c}{ Variable } & \multicolumn{1}{c}{ Frequency \%, Count (n=12,407) } \\
\hline Age & Median 61, Mean $58.5(21$ to 90) \\
\hline Gender & \\
Male & $93.0 \%(11543)$ \\
Female & $6.8 \%(864)$ \\
\hline Race & \\
American Indian or Alaska Native & $1.2 \%(147)$ \\
Asian & $<1 \%(43)$ \\
Black or African American & $21.8 \%(2706)$ \\
Native Hawaiian or Pacific Islander & $<1 \%(106)$ \\
White & $71.6 \%(8880)$ \\
Unknown/Declined to Answer & $4.2 \%(525)$ \\
\hline Ethnicity & \\
Hispanic or Latino & $4.8 \%(595)$ \\
Non-Hispanic or Latino & $92.5 \%(11476)$ \\
Unknown & $2.7 \%(336)$ \\
\hline
\end{tabular}

5.1.3. Admission characteristics. Admission-related characteristics were also homogenous. The median length of stay was 5 days ranging from 1 to 50 days. Most hospital admission sources were either direct admission ( $n=5,616 ; 45 \%)$ or from outpatient treatment ( $n=6,095 ; 49 \%)$. One in five patients $(n=2,303 ; 19 \%)$ received services in the intensive care unit (ICU) and 6\% $(n=779)$ received surgical services during their admission (see Table 5.3).

Table 5.3. Admission Characteristics

\begin{tabular}{ll}
\hline \multicolumn{1}{c}{ Variable } & \multicolumn{1}{c}{ Frequency \%, Count $(\mathbf{n}=\mathbf{1 2 , 4 0 7})$} \\
\hline Length of Stay & Median, 5; Mean 6.6 (1 - 50) \\
\hline Admission Source & \\
Outpatient Treatment & $49.1 \%(6095)$ \\
Other Direct Admission & $45.3 \%(5616)$ \\
Other & $4.9 \%(696)$ \\
\hline Service Acuity & \\
ICU Services & $18.6 \%(2303)$ \\
Surgical Services & $6.3 \%(779)$ \\
\hline
\end{tabular}

\subsubsection{Health characteristics during admission. Health-related characteristics}

during admission and present on admission were heterogenous. The prevalence of primary and secondary diagnosis admissions codes varied substantially with 2,150 
unique primary ICD-10 codes and 1,875 unique secondary ICD-10 codes in the study cohort. The top ten primary ICD-10 codes, which accounted for approximately $18 \%$ of admissions, included: 1) alcohol dependence (4\%); 2) chronic obstructive pulmonary disease with (acute) exacerbation (2\%); 3) opioid dependence with withdrawal $(2 \%) ; 4)$ sepsis, unspecified organism (2\%); 5) kidney failure, unspecified (1\%); 6) alcohol dependence with intoxication, unspecified (1\%); 7) other chest pain (1\%); 8) pneumonia, unspecified organism (1\%); 9) cellulitis of right lower limb (1\%); and 10) unilateral primary osteoarthritis, right knee (1\%).

The top ten secondary ICD-10 codes accounted for approximately $26 \%$ of unique admissions: 1) opioid dependence, uncomplicated (7\%); 2) acute kidney failure, unspecified (4\%); 3) suicidal ideations (4\%); 4) essential (primary) hypertension (2\%); 5) pneumonia, unspecified organism (2\%); 6) end stage renal disease (2\%); 7) human immunodeficiency virus disease $(2 \%) ; 8$ ) hypoosmolality and hyponatremia (1\%); 9) opioid dependence, with withdrawal (1\%); and 10) chronic obstructive pulmonary disease with acute exacerbations (1\%).

OUD-related infections occurred in 5.5\% of admissions. The top two OUD-related infections were osteomyelitis (2\%) and bacteremia (1\%). Acute OUD-related admission diagnoses occurred in $6 \%$ of primary diagnoses, $10 \%$ of secondary diagnoses, and $15 \%$ for either primary and/or secondary. Further, $20 \%$ of cases in the cohort had either an OUD infection flag or a primary or secondary OUD-related diagnosis (see Table 5.4). 
Table 5.4. Health-Related Characteristics: During Admission

\begin{tabular}{|c|c|c|c|}
\hline \multirow{2}{*}{\multicolumn{2}{|c|}{$\begin{array}{r}\text { Variable } \\
\text { Any Acute OUD Infection } \\
\end{array}$}} & \multicolumn{2}{|c|}{ Frequency $\%$, Count $(n=12,407)$} \\
\hline & & $5.5 \%$ & $(691)$ \\
\hline 1. & Osteomyelitis (M86.X) & $2.2 \%$ & $(274)$ \\
\hline 2. & Bacteremia (R78.81) & $1.3 \%$ & $(158)$ \\
\hline 3. & Epidural Abscess/ Diskitis (M46.4; M51.9; M50.X; M51.8) & $<1 \%$ & $(112)$ \\
\hline 4. & Septic Arthritis (M00.) & $<1 \%$ & (71) \\
\hline 5. & Prosthetic Joint Infection (T84.5x) & $<1 \%$ & (57) \\
\hline 6. & Endocarditis (I33.X) & $<1 \%$ & (46) \\
\hline 7. & Brain Abscess (G06.X) & $<1 \%$ & (45) \\
\hline 8. & Lung Abscess (J85) & $<1 \%$ & (38) \\
\hline & Empyema (J86) & $<1 \%$ & (24) \\
\hline & Candida Endocarditis (B37.6) & $<1 \%$ & (3) \\
\hline Any & OUD-Related Diagnosis Primary or Secondary ${ }^{b}$ & $14.9 \%$ & $(1848)$ \\
\hline Prin & nary & $5.7 \%$ & (707) \\
\hline $\mathrm{Sec}$ & ondary & $9.5 \%$ & $(1181)$ \\
\hline Any & OUD-Related Diagnosis or Any Acute OUD Infection & $20.1 \%$ & $(2491)$ \\
\hline \multicolumn{4}{|c|}{ Top 10 Primary ICD-10 Diagnosis Codes } \\
\hline 1. & Alcohol Dependence (F10.239) & $4.4 \%$ & $(551)$ \\
\hline 2. & $\begin{array}{l}\text { Chronic Obstructive Pulmonary Disease with (acute) } \\
\text { Exacerbation (J44.1) }\end{array}$ & $2.3 \%$ & $(289)$ \\
\hline 3. & Opioid Dependence with Withdrawal (F11.23) & $2.3 \%$ & $(281)$ \\
\hline 4. & Sepsis, Unspecified Organism (A41.9) & $1.7 \%$ & $(213)$ \\
\hline 5. & Acute Kidney Failure, Unspecified (N17.9) & $1.4 \%$ & $(174)$ \\
\hline & Alcohol Dependence with Intoxication, Unspecified (F10.229) & $1.4 \%$ & $(173)$ \\
\hline 7. & Other Chest Pain (R07.89) & $1.3 \%$ & $(165$ \\
\hline 8. & Pneumonia, Unspecified Organism (J18.9) & $1.2 \%$ & $(151)$ \\
\hline 9. & Cellulitis of Right Lower Limb (L03.115) & $1.1 \%$ & $(133)$ \\
\hline & Unilateral Primary Osteoarthritis, Right Knee (M17.11) & $1.0 \%$ & $(122)$ \\
\hline \multicolumn{4}{|c|}{ Top 10 Secondary ICD-10 Diagnosis Codes } \\
\hline & Opioid Dependence, Uncomplicated (F11.20) & $6.8 \%$ & $(842)$ \\
\hline & Acute Kidney Failure, Unspecified (N17.9) & $4.2 \%$ & $(526)$ \\
\hline 3. & Suicidal Ideations (R45.851) & $4.1 \%$ & $(508)$ \\
\hline 4. & Essential (Primary) Hypertension (I10.) & $2.1 \%$ & $(257)$ \\
\hline 5. & Pneumonia, Unspecified Organism (J18.9) & $1.6 \%$ & (193) \\
\hline & End Stage Renal Disease (N18.6) & $1.5 \%$ & $(191)$ \\
\hline & Human Immunodeficiency Virus [HIV] Disease (B20) & $1.5 \%$ & $(185)$ \\
\hline & Hypo-Osmolality and Hyponatremia (E87.1) & $1.3 \%$ & $(162)$ \\
\hline & Opioid Dependence with Withdrawal (F11.23) & $1.3 \%$ & (159) \\
\hline \multicolumn{4}{|c|}{ Exacerbation (J44.1) } \\
\hline
\end{tabular}

\subsubsection{Co-occurring conditions present on admission. Co-occurring diagnoses}

occurred within 365 days prior to the index hospitalization. Patients with chronic

cancer-related AHRQ Elixhauser flags (AHRQ, 2018) were excluded from the cohort 
because of the common clinical practice of prescribing opioids for this population

(Caraceni et al., 2012). Co-occurring mental health and SUD were present in more than half of the study cohort $-64 \%$ of cases had a mental health diagnosis and $49 \%$ had another SUD diagnosis. The most common mental health disorders present on admission were mood disorders (50\%), PTSD (31\%), and anxiety (28\%). The most common SUD diagnoses were alcohol use disorder (36\%), other psychoactive use disorders (17\%), and cannabis use disorder (16\%) (see Table 5.5).

Table 5.5. Health-Related Characteristics: Present on Admission

\begin{tabular}{|c|c|c|}
\hline Variable & Frequency $\%$, Count (n & $=12,407$ \\
\hline Co-Occurring Mental Health ${ }^{a}$ & $65.2 \%$ & (8094) \\
\hline 1. Mood Disorder & $50.8 \%$ & (6306) \\
\hline 2. PTSD & $31.8 \%$ & (3951) \\
\hline 3. Anxiety Disorder & $28.7 \%$ & $(3560)$ \\
\hline 4. Non-Mood Psychotic Disorder & $7.7 \%$ & (957) \\
\hline 5. Adjustment Disorder Other & $3.2 \%$ & $(402)$ \\
\hline 6. Self-Harm & $2.9 \%$ & $(358)$ \\
\hline Co-Occurring Substance Use Disorders ${ }^{a}$ & $48.6 \%$ & $(6024)$ \\
\hline 1. Alcohol Use Disorder & $37.4 \%$ & (4641) \\
\hline 2. Other Psychoactive Use Disorder & $17.3 \%$ & $(2147)$ \\
\hline 3. Cannabis Use Disorder & $16.5 \%$ & $(2045)$ \\
\hline 4. Stimulant Use Disorder & $9.5 \%$ & $(1172)$ \\
\hline 5. Cocaine Use Disorder & $6.2 \%$ & (769) \\
\hline 6. Nicotine Dependence & $3.5 \%$ & $(434)$ \\
\hline 7. Sedative Use Disorder & $2.9 \%$ & (354) \\
\hline 8. Other Substance Use Disorders & $1.9 \%$ & $(241)$ \\
\hline 9. Hallucinogen Use Disorders & $<1 \%$ & $(66)$ \\
\hline 10. Inhalant Related Use Disorders & $<1 \%$ & $(46)$ \\
\hline
\end{tabular}

5.1.6. Pharmacotherapy. The pharmacotherapy data were examined for the periods of 30 days pre-admission, admission, and 30 days post-admission.

5.1.6a. Pre-admission pharmacotherapy. Nearly one in three patients (30\%) filled at least one opioid prescription in the 30 days prior to index-hospitalization. The two most common opioid prescriptions were oxycodone (12\%) and hydrocodone (10\%). 
A prescription for gabapentin/pregabalin (e.g., Lyrica ${ }^{\circledR}$ ) was filled in $32 \%$ of unique cases and benzodiazepine prescriptions were present in $9 \%$ of cases (see Table 5.6).

Table 5.6. Pre-Admission: Non-OAT Pharmacotherapy ${ }^{a}$

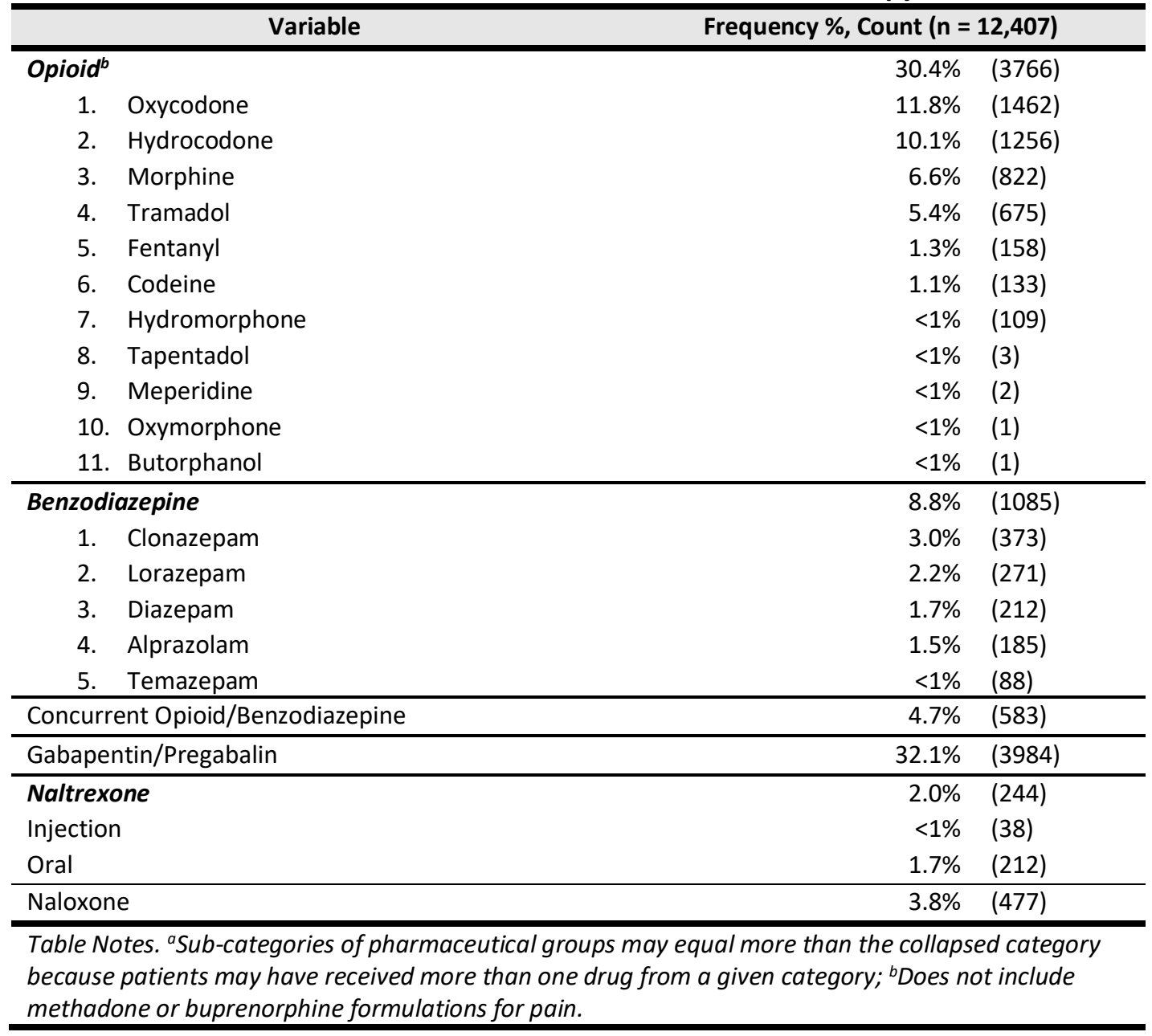

OAT receipt occurred in $11 \%$ of cases $(n=1,325)$ in the pre-admission time period. The most common type of OAT was buprenorphine $(5 \% ; n=625)$ and the second most common was non-specific OAT administration (4.7\%; $n=557)$ (see Table 5.7). 
Table 5.7. Pre-Admission: OAT

\begin{tabular}{|c|c|c|}
\hline Variable & Frequency $\%$, Count ( $r$ & $=12,407)$ \\
\hline Any $O A T^{a}$ & $10.7 \%$ & (1325) \\
\hline Buprenorphine Only ${ }^{b}$ & $5.0 \%$ & $(625)$ \\
\hline Non-Specific Administration ${ }^{c}$ & $4.7 \%$ & (577) \\
\hline$>$ 1Type of OAT Received ${ }^{d}$ & $<1 \%$ & $(112)$ \\
\hline Methadone Onlye & $<1 \%$ & (11) \\
\hline \multicolumn{3}{|c|}{$\begin{array}{l}\text { Table Notes. }{ }^{a} \text { Includes each of the } 4 \text { sub-categories in this table; }{ }^{b} \text { Includes buprenorphine prescription } \\
\text { fills and the Healthcare Common Procedure Coding System (HCPCS) codes (JO574, J0575, J0571); } \\
\text { 'Includes an OTP stop code visit or the non-specific OAT administration HCPCS code (H0033); }{ }^{d} \text { Includes } \\
\text { any patients who received more than one type of OAT during the pre-period; }{ }^{e} \text { Includes the methadone } \\
\text { specific HCPCS code (SO109). }\end{array}$} \\
\hline
\end{tabular}

5.1.6b. Admission pharmacotherapy. Admission data suggested frequent opioid

administration during admission. More than half of patients (55\%) had at least one opioid delivered during admission; oxycodone (29\%) and morphine (20\%) were most common. More than one in three admissions received gabapentin/pregabalin (37\%) and more than one in four admissions received benzodiazepines (30\%). First or second-line adjuvants for the symptom management of opioid withdrawal were administered in $44 \%$ of cases. The VHA's recommended first line adjuvant for withdrawal, clonidine, was only observed in $9 \%$ of cases; second-line adjuvants (i.e., baclofen, gabapentin, pregabalin, or tizanidine) were present in $39 \%$ of cases (see Table 5.8). 
Table 5.8. Admission: Non-OAT Pharmacotherapy ${ }^{\mathrm{a}}$

\begin{tabular}{|c|c|c|}
\hline \multirow{2}{*}{$\begin{array}{ll}\text { Any Opioid } & \text { Variable } \\
\end{array}$} & \multicolumn{2}{|c|}{ Frequency $\%$, Count $(n=12,407)$} \\
\hline & $54.5 \%$ & $(6765)$ \\
\hline 1. Oxycodone & $29.2 \%$ & (3623) \\
\hline 2. Morphine & $20.4 \%$ & $(2525)$ \\
\hline 3. Hydromorphone & $18.0 \%$ & $(2635)$ \\
\hline 4. Hydrocodone & $13.3 \%$ & $(1645)$ \\
\hline 5. Tramadol & $8.1 \%$ & $(1008)$ \\
\hline 6. Fentanyl & $4.4 \%$ & $(551)$ \\
\hline 7. Codeine & $<1 \%$ & $(103)$ \\
\hline 8. Meperidine & $<1 \%$ & (17) \\
\hline 9. Belladonna & $<1 \%$ & $(11)$ \\
\hline 10. Tapentadol & $<1 \%$ & $(2)$ \\
\hline 11. Oxymorphone & $<1 \%$ & $(1)$ \\
\hline 12. Butorphanol & $<1 \%$ & $(1)$ \\
\hline No Opioids and No OAT & $35.6 \%$ & (4418) \\
\hline Any adjuvants & $44.4 \%$ & $(5502)$ \\
\hline First-Line Adjuvant ${ }^{c}$ & $8.8 \%$ & (1089) \\
\hline Any Second-Line Adjuvant ${ }^{d}$ & $39.3 \%$ & $(4882)$ \\
\hline Any benzodiazepine & $30.3 \%$ & $(3757)$ \\
\hline 1. Lorazepam & $21.6 \%$ & $(2677)$ \\
\hline 2. Diazepam & $5.3 \%$ & $(662)$ \\
\hline 3. Clonazepam & $3.1 \%$ & $(383)$ \\
\hline 4. Alprazolam & $1.9 \%$ & $(240)$ \\
\hline 5. Midazolam & $1.7 \%$ & $(214)$ \\
\hline 6. Temazepam & $1.3 \%$ & $(159)$ \\
\hline Gabapentin/Pregabalin & $36.7 \%$ & $(4556)$ \\
\hline Naltrexone & $1.4 \%$ & $(168)$ \\
\hline Oral Tablet & $1.3 \%$ & $(155)$ \\
\hline Injectable & $<1 \%$ & (31) \\
\hline $\begin{array}{l}\text { Table Notes. }{ }^{a} \text { Group sub-categories } \\
\text { can receive more than one drug fro } \\
\text { formulations; }{ }^{c} \text { First-line: clonidine; }\end{array}$ & $\begin{array}{l}\text { apsed category becau } \\
\text { le methadone or bupr } \\
\text { entin, pregabalin, and }\end{array}$ & $\begin{array}{l}\text { e patients } \\
\text { norphine } \\
\text { izanidine. }\end{array}$ \\
\hline
\end{tabular}

OAT was administered in $15 \%$ of cases, with methadone delivery (9\%) more

common than buprenorphine (5\%). Across the study population, OAT was used primarily to manage withdrawal symptoms (7\%) (i.e., the patient did not receive OAT prior to or after admission, only during admission). The second most common OAT delivery mechanism was OAT sustained (6\%) (i.e., a patient received OAT throughout the care continuum -in the pre, admission, and post periods). OAT initiation and linkage to care (2\%) were uncommon (i.e., OAT starting in the hospital and continued in the outpatient 
setting within 30 rdays of discharge). Very few patients received OAT continuation (1\%)

(i.e., OAT delivery in the pre-period and during admission, but not in the post-admission discharge period) (see Table 5.9).

Table 5.9. Admission: OAT

\begin{tabular}{|c|c|c|}
\hline \multirow[t]{2}{*}{ Variable } & \multicolumn{2}{|c|}{ Frequency $\%$, Count $(n=12,407)$} \\
\hline & $15.4 \%$ & (1914) \\
\hline Methadone Only ${ }^{b}$ & $8.5 \%$ & (1049) \\
\hline Buprenorphine Onlyc & $5.2 \%$ & (639) \\
\hline$>1$ Type of OAT Received ${ }^{d}$ & $1.1 \%$ & $(136)$ \\
\hline Non-Specific Administration Onlye & $<1 \%$ & (90) \\
\hline \multicolumn{3}{|l|}{ OAT by Care Delivery Scenario } \\
\hline OAT Withdrawal ${ }^{f}$ & $6.8 \%$ & $(844)$ \\
\hline OAT Sustainedg & $5.8 \%$ & $(722)$ \\
\hline OAT Initiation and Linkage ${ }^{h}$ & $1.6 \%$ & $(203)$ \\
\hline OAT Continuedi & $1.2 \%$ & $(145)$ \\
\hline \multicolumn{3}{|c|}{ OAT Administered with Other Pharmacotherapy } \\
\hline With Any Adjuvant & $7.2 \%$ & $(893)$ \\
\hline With Second-Line Adjuvant & $6.2 \%$ & $(766)$ \\
\hline With Opioid & $5.6 \%$ & $(690)$ \\
\hline With First-Line Adjuvant: Clonidine & $2.2 \%$ & $(271)$ \\
\hline With Naltrexone & $<1 \%$ & (9) \\
\hline \multicolumn{3}{|c|}{$\begin{array}{l}\text { ancludes each of the } 4 \text { OAT sub-categories in this table; }{ }^{b} \text { Includes the methadone specific Healthcare } \\
\text { Common Procedure Coding System (HCPCS) code (S0109); 'Includes buprenorphine prescription fills } \\
\text { and HCPCS codes (J0574, J0575, J0571); }{ }^{d} \text { Includes any patients who received more than one type of } \\
\text { OAT during admission; }{ }^{e} \text { Includes an OTP stop code visit or the non-specific OAT administration HCPCS } \\
\text { code (HO033); } \text { OAT received during admission, but not in pre or post period; }{ }^{g} O A T \text { received in the pre, } \\
\text { admission, and post periods; }{ }^{h} \text { OAT received during admission and the post period, but not the pre } \\
\text { period; 'OAT received in the pre and admission periods, but not the post period. }\end{array}$} \\
\hline
\end{tabular}

5.1.6c. Post-admission pharmacotherapy. Over one third of the patients (34\%) in the study cohort filled one or more opioid-related prescriptions in the 30 days after hospital discharge. The most commonly filled prescriptions were oxycodone $(17 \%)$ and hydrocodone (10\%). Compared with the pre-admission data, the proportion of cases with gabapentin/pregabalin (26\%) and benzodiazepine (8\%) prescriptions was lower. Compared with the pre-admission data, the proportion of cases prescribed naloxone (6\%) post-admission was more common than pre-admission (4\%) (see Table 5.10). 
Table 5.10. Post-Admission: Non-OAT Pharmacotherapy ${ }^{\mathrm{a}}$

\begin{tabular}{|c|c|c|}
\hline \multirow{2}{*}{$\begin{array}{lr}\text { Variable } \\
\text { Any Opioid }^{b}\end{array}$} & \multicolumn{2}{|c|}{ Frequency $\%$, Count $(n=12,407)$} \\
\hline & $34.3 \%$ & (4250) \\
\hline 1. Oxycodone & $17.0 \%$ & $(2112)$ \\
\hline 2. Hydrocodone & $9.9 \%$ & (1229) \\
\hline 3. Morphine & $6.1 \%$ & (759) \\
\hline 4. Tramadol & $5.2 \%$ & $(650)$ \\
\hline 5. Hydromorphone & $1.6 \%$ & $(196)$ \\
\hline 6. Fentanyl & $1.2 \%$ & $(150)$ \\
\hline 7. Codeine & $<1 \%$ & $(108)$ \\
\hline 8. Tapentadol & $<1 \%$ & $(2)$ \\
\hline 9. Meperidine & $<1 \%$ & $(2)$ \\
\hline 10. Oxymorphone & $<1 \%$ & (1) \\
\hline 11. Belladonna & $<1 \%$ & $(1)$ \\
\hline Any Benzodiazepine & $7.7 \%$ & (949) \\
\hline 1. Clonazepam & $2.3 \%$ & $(317)$ \\
\hline 2. Lorazepam & $2.2 \%$ & $(267)$ \\
\hline 3. Diazepam & $1.5 \%$ & (188) \\
\hline 4. Alprazolam & $1.1 \%$ & $(140)$ \\
\hline 5. Temazepam & $<1 \%$ & (66) \\
\hline 6. Midazolam & $<1 \%$ & (3) \\
\hline Concurrent Opioid \& Benzodiazepine & $3.9 \%$ & $(483)$ \\
\hline Gabapentin/Pregabalin & $26.3 \%$ & (3263) \\
\hline Naltrexone & $2.8 \%$ & (341) \\
\hline Oral Tablet & $2.3 \%$ & $(289)$ \\
\hline Injectable & $<1 \%$ & $(68)$ \\
\hline Naloxone & $6.2 \%$ & $(765)$ \\
\hline $\begin{array}{l}\text { Table Notes. }{ }^{a} \text { Sub-categories of pharm } \\
\text { category because patients may have r } \\
{ }^{b} \text { Does not include methadone or bupre }\end{array}$ & $\begin{array}{l}\text { equal more than th } \\
\text { ne drug from a given } \\
\text { ns for pain. }\end{array}$ & $\begin{array}{l}\text { collapsed } \\
\text { ategory; }\end{array}$ \\
\hline
\end{tabular}

OAT receipt during the post-admission period occurred in $11 \%$ of cases, with a nearly even distribution of use between buprenorphine (5\%) and non-specific administration (5\%) (see Table 5.11). 
Table 5.11. Post-Admission: OAT

\begin{tabular}{|c|c|c|}
\hline Variable & Frequency $\%$, Cou & nt $(n=12,407)$ \\
\hline Any $O A T^{a}$ & $11.4 \%$ & $(1420)$ \\
\hline Buprenorphine Only ${ }^{b}$ & $5.1 \%$ & (633) \\
\hline Non-Specific Administration Onlyc & $5.1 \%$ & $(628)$ \\
\hline$>1$ Type of OAT Received ${ }^{d}$ & $1.1 \%$ & $(142)$ \\
\hline Methadone Only & $<1 \%$ & (17) \\
\hline \multicolumn{3}{|c|}{$\begin{array}{l}\text { Table Notes. }{ }^{a} \text { Includes each of the } 4 \text { sub-categories in this table; }{ }^{b} \text { Includes buprenorphine prescription } \\
\text { fills and the Healthcare Common Procedure Coding System (HCPCS) codes (J0574, J0575, J0571); } \\
\text { 'Includes an OTP stop code visit or the non-specific OAT administration HCPCS code (H0033); }{ }^{d} \text { Includes } \\
\text { any patients who received more than one type of OAT during admission; }{ }^{e} \text { Includes the methadone } \\
\text { specific HCPCS code (S0109). }\end{array}$} \\
\hline
\end{tabular}

5.1.7. Admission-related outcomes. The admission-related outcomes were assessed during hospitalization or within the 30 days post-admission. In-hospital mortality (1\%), post-admission death $(<1 \%)$, and leaving against medical advice (6\%) were uncommon outcomes across the study cohort. In contrast, it was common for patients to need additional acute care services during the post-admission period. More than one quarter of patients (28\%) in the sample had an emergency department visit, and re-admissions occurred for $13 \%$ of patients (see Table 5.12 ).

Table 5.12. VHA Study Cohort Outcomes

\begin{tabular}{lc}
\hline \multicolumn{1}{c}{ Variable } & Frequency \%, Count $(\mathbf{n}=\mathbf{1 2 , 4 0 7 )}$ \\
\hline Admission & \\
Left Against Medical Advice & $5.7 \%(701)$ \\
In-Hospital Mortality & $1.0 \%(119)$ \\
\hline Post-Admission & \\
Death & $<1 \%(112)$ \\
Emergency Department Visit & $27.7 \%(3436)$ \\
Hospital Readmission & $13.1 \%(1630)$ \\
\hline
\end{tabular}

5.1.8. Summary. This section explored the supply-side and demand-side attributes, care delivery patterns, and outcomes for the entire study cohort. The study cohort characteristics were homogenous for the supply-side (i.e., hospitals) and heterogenous for demand-side attributes (i.e., patients). Most admissions were for 
elderly white men, with co-occurring mental health conditions, in large hospitals. In contrast, the clinical reasons for admission varied among patients. Interestingly, most admissions were not OUD-related by primary or secondary diagnosis or by OUD-related infection codes.

These descriptive analyses begin to depict care patterns for patients with OUD that are potentially problematic. In all three time periods (pre, admission, post), opioid prescription or administration frequency was higher than OAT delivery. When OAT was delivered in the hospital setting, it was primarily administered for withdrawal management. The most effective approaches to care (i.e., OAT sustaining, continuing, initiating and linkage to care) occurred infrequently. An additional indicator of poor care or lower quality care delivery in the hospital setting was the elevated use of the emergency department in the post-admission period. This measure has not been studied in the VHA system; however, a 30-day hospital readmission rate for the VHA system has been reported at 18\% (Vincent, Wiitala, Burns, Iwashyna, \& Prescott, 2018), and a previous hospitalization $(\mathrm{OR}, 2.5 ; 95 \% \mathrm{Cl}[2.3,2.7])$ is associated with increased risk of an emergency department visit (Hastings et al., 2011).

\section{Section 5.2. OAT vs. No OAT Received During Admission}

To expand upon the observations of the prior section, this section explored potential differences between patients who received or did not receive OAT based on clinical and admission characteristics, care delivery patterns, and outcomes. This section addressed the following research sub-questions: 
- 2a: Do the characteristics of the patients who received OAT differ from those who do not receive OAT while hospitalized?

- $2 b$ : Does non-OAT pharmacotherapy delivery differ for patients who received OAT and for those who did not receive OAT while hospitalized?

- 2c: Do outcomes differ for patients who received OAT and for those who did not receive OAT while hospitalized?

5.2.1. Patient characteristics. Patients who received OAT differed statistically ( $p$ $<0.05)$ from those who did not receive OAT during admission. OAT patients were one year younger ( mean $=57$ years of age vs. 58 years of age) and were less likely to be women ( $5 \%$ vs. $7 \%$ ). Patients who received OAT had a statistically significant different distribution of racial categories with more Black patients ( $27 \%$ vs. $21 \%$ ) and fewer White patients (68\% vs. 76\%). Patients administered OAT during hospitalization were also more likely to have comorbid SUDs ( $51 \%$ vs. $48 \%$ ) and to have mental health disorders (67\% vs. $65 \%$ ). Although the two groups differed statistically on most characteristics, the differences do not appear to be clinically significant; rather they reflect the study's large sample size (see Table 5.13). 
Table 5.13. Patient Characteristics: OAT Received vs. Not Received

\begin{tabular}{|c|c|c|c|c|}
\hline Characteristics & $\begin{array}{c}\text { OAT Received } \\
(15 \%, 1,914)\end{array}$ & $\begin{array}{c}\text { No OAT Received } \\
(85 \%, 10,597)\end{array}$ & Difference & $\begin{array}{c}\text { Test } \\
\text { Statistic }\end{array}$ \\
\hline Age $* * *$ & $\begin{array}{l}\text { Median, } 60.0 \text { years } \\
\text { Mean, } 57.1 \\
(\mathrm{SD}=12.4 ; 23-90)\end{array}$ & $\begin{array}{l}\text { Median, } 61.0 \text { years } \\
\text { Mean, } 58.8 \\
(\mathrm{SD}=13.4 ; 21-90)\end{array}$ & 1 year & $\begin{array}{l}W=10675000 \\
p<0.05^{a}\end{array}$ \\
\hline \multicolumn{5}{|l|}{$\operatorname{Race}^{* * *}$} \\
\hline American Indian or Alaska Native & $<1 \%(12)$ & $1.3 \%(135)$ & $\approx<1 \%$ & $X^{2}=42.56$ \\
\hline Asian & $<1 \%(7)$ & $<1 \%(36)$ & $\approx<1 \%$ & $\mathrm{df}=5$ \\
\hline Black or African American & $27.0 \%(516)$ & $21.0 \%(2190)$ & $\approx 6 \%$ & $\mathrm{p}<0.05^{\mathrm{b}}$ \\
\hline Native Hawaiian or Pacific Islander & $<1 \%(12)$ & $<1 \%(94)$ & $\approx<1 \%$ & \\
\hline Unknown/Declined to Answer & $3.4 \%(65)$ & $4.3 \%(460)$ & $\approx<1 \%$ & \\
\hline White & $68.0 \%(1302)$ & $75.7 \%(7578)$ & $\approx 7 \%$ & \\
\hline \multicolumn{5}{|l|}{ Ethnicity } \\
\hline Hispanic or Latino & $5.5 \%(105)$ & $4.7 \%(490)$ & $\approx<1 \%$ & $X^{2}=2.3663$ \\
\hline Non-Hispanic or Latino & $91.8 \%(1758)$ & $92.6 \%(9718)$ & $\approx<1 \%$ & $\mathrm{df}=2$ \\
\hline Unknown & $2.6 \%(51)$ & $2.8 \%(285)$ & $\approx<1 \%$ & $p>0.05$ \\
\hline \multicolumn{5}{|l|}{ Gender*** } \\
\hline Male & $95.5 \%(1827)$ & $92.6 \%(9716)$ & $\approx 2 \%$ & $X^{2} 19.99$ \\
\hline Female & $4.6 \%(87)$ & $7.4 \%$ (777) & $\approx 3 \%$ & $\begin{array}{l}\mathrm{df}=1 \\
\mathbf{p}<\mathbf{0 . 0 5 ^ { c }}\end{array}$ \\
\hline \multicolumn{5}{|l|}{ Comorbidities } \\
\hline SUD* & $51.4 \%(983)$ & $48.0 \%(5041)$ & $\approx 3 \%$ & $\begin{array}{l}X^{2}=6.9972 \\
d f=1 \\
p<0.05^{c}\end{array}$ \\
\hline Mental health condition** & $67.4 \%(1290)$ & $64.8 \%(6804)$ & $\approx 2 \%$ & $\begin{array}{l}X^{2}=4.5472 \\
d f=1 \\
p<0.05^{c}\end{array}$ \\
\hline
\end{tabular}


5.2.2. Admission characteristics. The two groups had statistically significant differences for length of stay, admission source, acuity of services received (ICU and surgical), OUD diagnoses, and OUD infection diagnoses. Although, again, most observed differences between the two groups were likely trivial, findings with substantive relevance were likely for three characteristics. Patients who received OAT had a longer length of stay ( 5 days vs. 4 days) and were more likely to have a primary or secondary OUD diagnosis ( $29 \%$ vs. $12 \%$ ) or an OUD diagnosis/and or an OUD-related infection (37\% vs. $17 \%$ ) (see Table 5.14). 
Table 5.14. Admission-Related Characteristics: OAT Received vs. Not Received

\begin{tabular}{|c|c|c|c|c|}
\hline $\begin{array}{c}\text { Admission-Related } \\
\text { Variables }\end{array}$ & $\begin{array}{l}\text { OAT Received } \\
(15 \%, 1,914)\end{array}$ & $\begin{array}{c}\text { No OAT Received } \\
(85 \%, 10,597)\end{array}$ & Difference & $\begin{array}{c}\text { Test } \\
\text { Statistic }\end{array}$ \\
\hline Length of Stay*** & $\begin{array}{l}\text { Median, } 5 \text { days } \\
\text { Mean, } 7.53 \\
(\text { SD } 7.05 ; 1-50) \\
\end{array}$ & $\begin{array}{l}\text { Median, } 4 \text { days } \\
\text { Mean, } 6.44 \\
\text { (SD 6.23, } 1-50) \\
\end{array}$ & 1 day & $\begin{array}{l}W=8758700 \\
p<0.05^{a}\end{array}$ \\
\hline \multicolumn{5}{|l|}{ Admission Source $* * *$} \\
\hline Outpatient Treatment & $46.0 \%(880)$ & $49.7 \%(5215)$ & $\approx 4 \%$ & $X^{2}=13.533$ \\
\hline Other Direct Admission & $49.3 \%(942)$ & $44.5 \%(4673)$ & $\approx 4 \%$ & $\mathrm{df}=2$ \\
\hline Other & $4.8 \%(91)$ & $5.8 \%(605)$ & $\approx 1 \%$ & $\mathrm{p}<0.05^{\mathrm{b}}$ \\
\hline \multicolumn{5}{|l|}{ Type of Services Received } \\
\hline ICU Services** & $16.3 \%(312)$ & 19.0\% (1991) & $\approx 3 \%$ & $\begin{array}{l}X^{2}=7.4787 \\
d f=1 \\
p<0.05^{c}\end{array}$ \\
\hline Surgical Services* & $5.2 \%(100)$ & $6.5 \%(679)$ & $\approx<1 \%$ & $\begin{array}{l}X^{2}=4.0638 \\
d f=1 \\
p<0.05^{c}\end{array}$ \\
\hline \multicolumn{5}{|l|}{ Acute OUD or Infection Diagnosis } \\
\hline Acute OUD Diagnosis*** & $29.0 \%(560)$ & $12.3 \%(1288)$ & $\approx 17 \%$ & $\begin{array}{l}X^{2}=366.98 \\
d f=1 \\
p<0.05^{c}\end{array}$ \\
\hline OUD Infection Diagnosis *** & $8.3 \%(158)$ & $5.1 \%(533)$ & $\approx 3 \%$ & $\begin{array}{l}x^{2}=30.434 \\
d f=1 \\
p<0.05^{c}\end{array}$ \\
\hline Acute OUD or Infection Diagnosis**** & $36.5 \%(698)$ & $17.1 \%(1793)$ & $\approx 20 \%$ & $\begin{array}{l}X^{2}=377.7 \\
d f=1 \\
p<0.05^{c}\end{array}$ \\
\hline
\end{tabular}


5.2.3. Care delivery during admission. Care delivery processes during admission differed significantly for patients who received OAT and for those who did not. Patients who received OAT were less likely to receive opioids (36\% vs. $58 \%$ ), benzodiazepines ( $28 \%$ vs. $31 \%)$, or naltrexone ( $<1 \%$ vs. $2 \%)$. They were also more likely to receive withdrawal management adjuvants ( $48 \%$ vs. $44 \%$ ), or first-line adjuvants ( $14 \%$ vs. $8 \%$ ). The differences in opioid administration between the two groups were likely meaningful, although, generally, the remainder of the statistical differences appeared to have minimal clinical relevance (see Table 5.15).

Table 5.15. Admission Non-OAT Pharmacotherapy: OAT Received vs. Not Received

\begin{tabular}{|c|c|c|c|c|}
\hline $\begin{array}{c}\text { Care Delivery } \\
\text { Variables }\end{array}$ & $\begin{array}{c}\text { OAT Received } \\
(15 \%, 1,914)\end{array}$ & $\begin{array}{c}\text { No OAT Received } \\
(85 \%, 10,597)\end{array}$ & Difference & $\begin{array}{c}\text { Test } \\
\text { Statistic }\end{array}$ \\
\hline Non-OAT Opioid*** & $36.1 \%(690)$ & $57.9 \%(6075)$ & $\approx 22 \%$ & $\begin{array}{l}X^{2}=310.67 \\
d f=1 \\
p<0.05^{c}\end{array}$ \\
\hline Any Adjuvant*** & $48.0 \%(919)$ & $43.7 \%(4583)$ & $\approx 4 \%$ & $\begin{array}{l}X^{2}=12.167 \\
d f=1 \\
p<0.05^{c}\end{array}$ \\
\hline $\begin{array}{l}\text { First-Line Adjuvant: } \\
\text { Clonidine*** }\end{array}$ & $14.2 \%(271)$ & $7.8 \%(818)$ & $\approx 7 \%$ & $\begin{array}{l}X^{2}=81.064 \\
d f=1 \\
p<0.05^{c}\end{array}$ \\
\hline Second-Line Adjuvant & $40.0 \%(766)$ & $39.2 \%(4116)$ & $\approx<1 \%$ & $\begin{array}{l}X^{2}=0.39576 \\
d f=1 \\
p>0.05^{c}\end{array}$ \\
\hline Benzodiazepine*** & $27.6 \%(528)$ & $30.8 \%(3229)$ & $\approx 3 \%$ & $\begin{array}{l}x^{2}=7.6361 \\
d f=1 \\
p<0.05^{c}\end{array}$ \\
\hline Naltrexone*** & $<1 \%(9)$ & $1.5 \%(159)$ & $\approx 1 \%$ & $\begin{array}{l}X^{2}=12.465 \\
d f=1 \\
p<0.05^{c}\end{array}$ \\
\hline
\end{tabular}

5.2.4. Health and utilization outcomes. Compared to patients who did not receive OAT, patients who received OAT were significantly less likely to leave against medical advice ( $4 \%$ vs. $6 \%$ ), and to receive post-admission prescriptions for opioids ( $16 \%$ 
vs. $38 \%$ ), benzodiazepines ( $5 \%$ vs. $8 \%$ ), concurrent opioids and benzodiazepines (<1\% vs. $4 \%)$, and naltrexone ( $<1 \%$ vs. $3 \%)$. The differences were small and likely have little clinical importance; however, two of the differences had apparent clinical significance: filled opioid prescriptions post-admission and OAT receipt post-admission (see Table 5.16). 
Table 5.16. Outcomes: OAT Received vs. Not Received

\begin{tabular}{|c|c|c|c|c|}
\hline $\begin{array}{l}\text { Outcome } \\
\text { Variables }\end{array}$ & $\begin{array}{c}\text { OAT Received } \\
(15 \%, 1,914)\end{array}$ & $\begin{array}{c}\text { No OAT Received } \\
(85 \%, 10,493)\end{array}$ & Difference & Test Statistic \\
\hline \multicolumn{5}{|l|}{ Admission } \\
\hline In-Hospital Mortality & $<1 \%(15)$ & $1.0 \%(104)$ & $\approx<1 \%$ & $\begin{array}{l}X^{2}=0.53115 \\
d f=1 \\
p>0.0\end{array}$ \\
\hline Left Against Medical Advice*** & $3.5 \%(68)$ & $6.0 \%(633)$ & $\approx 2 \%$ & $\begin{array}{l}X^{2}=18.211 \\
d f=1 \\
p<0.05\end{array}$ \\
\hline \multicolumn{5}{|l|}{ Post-Admission } \\
\hline Death & $<1 \%(18)$ & $<1 \%(94)$ & $\approx<1 \%$ & $\begin{array}{l}X^{2}=0.00343 \\
d f=1 \\
p>0.05\end{array}$ \\
\hline Emergency Department Visit & $26.1 \%(499)$ & $28.0 \%(2937)$ & $\approx 2 \%$ & $\begin{array}{l}X^{2}=2.8819 \\
d f=1 \\
p>0.05\end{array}$ \\
\hline Hospital Readmission & $12.8 \%(245)$ & $13.2 \%(1385)$ & $\approx<1 \%$ & $\begin{array}{l}X^{2}=0.19206 \\
d f=1 \\
p>0.05\end{array}$ \\
\hline Non-OAT Opioid*** & $16.3 \%(312)$ & $37.5 \%(3938)$ & $\approx 22 \%$ & $\begin{array}{l}X^{2}=322.98 \\
d f=1 \\
p<0.05\end{array}$ \\
\hline Benzodiazepine $* * *$ & $5.4 \%(103)$ & $8.1 \%(846)$ & $\approx 3 \%$ & $\begin{array}{l}X^{2}=16.095 \\
d f=1 \\
p<0.05\end{array}$ \\
\hline Naloxone*** & $11.2 \%(214)$ & $5.3 \%(551)$ & $\approx 6 \%$ & $\begin{aligned} X^{2} & =97.351 \\
d f & =1 \\
p & <0.05\end{aligned}$ \\
\hline Gabapentin/Pregabalin & $25.8 \%(493)$ & $26.4 \%(2770)$ & $\approx<1 \%$ & $\begin{array}{l}X^{2}=0.31084 \\
d f=1 \\
p>0.05\end{array}$ \\
\hline Naltrexone*** & $<1 \%(17)$ & $3.2 \%(324)$ & $\approx 3 \%$ & $\begin{array}{l}X^{2}=28.483 \\
d f=1 \\
p<0.05\end{array}$ \\
\hline $\begin{array}{l}\text { Concurrent Opioid/ } \\
\text { Benzodiazepine*** }\end{array}$ & $<1 \%(34)$ & $4.3 \%(449)$ & $\approx 4 \%$ & $\begin{array}{l}X^{2}=26.562 \\
d f=1 \\
p<0.05\end{array}$ \\
\hline OAT*** & $48.3 \%(925)$ & $4.7 \%(495)$ & $\approx 43 \%$ & $\begin{array}{l}X^{2}=3033.3 \\
d f=1 \\
p<0.05\end{array}$ \\
\hline
\end{tabular}

Table Notes. ${ }^{b}$ Pearson chi-square test; $p<0.05^{*} ; p<0.01^{* *} ; p<0.001^{* * *}$; Post-admission event within 30 days of discharge from index hospitalization. 
5.2.5. Summary. Most of the statistically significant findings were based on small relative differences that were likely not clinically significant but reflected the large study sample size. Four findings from this section stood out and warrant further discussion and exploration. First, patients who received OAT were more likely to have an OUD diagnosis and/or an OUD-related infection. This suggests that treatment processes and services differed between patients with an acute OUD-related issue during admission as compared with patients with a chronic OUD presentation. Second, patients who received OAT had a lower frequency of opioid administration during hospitalization. Determining whether this is appropriate or inappropriate care is challenging without further granularity on each case. The qualitative analyses in Chapter 4 suggested that patients who received OAT in the hospital setting may require additional opioids to manage pain when OAT administration alone is insufficient to manage moderate to severe pain. Lower opioid administration for this patient population during hospitalization, therefore, may be inappropriate. Third, patients who received OAT had less post-admission opioid prescriptions filled suggesting that prescribers may be assessing opioid risk more carefully among patients who received OAT. However, $16 \%$ of these patients were still receiving non-OAT opioids in the post-admission period. Fourth, patients who received OAT were more likely to receive OAT in the 30-day discharge period suggesting that OAT delivery in the hospital setting is part of a broader pathway and continuum of care for treating patients with OUD in the VHA system. 


\section{Section 5.3. OAT Discontinuation vs. OAT Continuation Upon Admission}

There were 1,325 patients in the sample receiving OAT prior to hospital admission. Of those patients, $65 \%(n=867)$ continued OAT during admission, and $35 \%$ ( $n=458$ ) were discontinued from OAT during admission. Of the patients who continued OAT during admission, most (83\%) were continued in the post-admission period $(\mathrm{n}=$ 722; the OAT sustained variable). Of those who were discontinued upon admission, $64 \%$ of those patients ( $n=295$; the OAT discontinued with restart variable) were restarted upon discharge in the post-admission period. Post-hospitalization approximately $23 \%$ of patients ( $n=308$, the OAT interrupted variable) who had received OAT prior to hospital admission (regardless of care received during admission) had their OAT care interrupted by hospitalization, and did not return to OAT in the 30 days post-admission. Section 5.3 addresses the following research sub-questions:

- $2 \mathrm{~d}$ : Do the characteristics of the patients who were discontinued from OAT differ from those who were continued on OAT at admission?

- 2e: Does non-OAT pharmacotherapy delivery differ for patients who received OAT and for those who did not receive OAT while hospitalized?

- 2f: Do outcomes differ for patients who received OAT and for those who did not receive OAT while hospitalized?

5.3.1. Patient characteristics. Patients who were discontinued on OAT upon admission $(n=458)$ and patients who continued OAT during admission $(n=867)$ differed. The discontinued patients were younger (mean of 54 years of age vs. 56 years 
of age) and more likely to be women ( $9 \%$ vs. $4 \%$ ). The observed differences were not large, and are not clearly clinically significant, but may reflect some combined demographics or a sub-population not otherwise discernable in the bivariate analysis (see Table 5.17). 
Table 5.17. Patient Characteristics: OAT Continued vs. Discontinued

\begin{tabular}{|c|c|c|c|c|}
\hline Characteristics & $\begin{array}{l}\text { OAT Discontinued } \\
\qquad(n=458)\end{array}$ & $\begin{array}{l}\text { OAT Continued } \\
\qquad(n=867)\end{array}$ & Difference & $\begin{array}{c}\text { Test } \\
\text { Statistic }\end{array}$ \\
\hline Age** & $\begin{array}{l}\text { Median, } 59.0 \text { years } \\
\text { Mean, } 54.0 \\
(\mathrm{SD}=13.2 ; 24-81)\end{array}$ & $\begin{array}{l}\text { Median, } 60.0 \text { years } \\
\text { Mean, } 56.2 \\
(\mathrm{SD}=12.4 ; 23-87)\end{array}$ & 1 year & $\begin{array}{l}W=216700 \\
p<0.05^{a}\end{array}$ \\
\hline \multicolumn{5}{|l|}{ Race } \\
\hline American Indian/Alaska Native & $<1 \%(1)$ & $<1 \%(3)$ & $\approx<1 \%$ & $X^{2}=6.108$ \\
\hline Asian & $<1 \%(2)$ & $<1 \%(3)$ & $\approx<1 \%$ & $\mathrm{df}=5$ \\
\hline Black/African American & $22.3 \%(102)$ & $28.1 \%(244)$ & $\approx 6 \%$ & $p>0.05^{b}$ \\
\hline Native Hawaiian/Pacific Islander & $<1 \%(2)$ & $<1 \%(6)$ & $\approx<1 \%$ & \\
\hline Unknown/Declined to Answer & $2.8 \%(13)$ & $2.8 \%(24)$ & No diff. & \\
\hline White & $73.8 \%(338)$ & $67.7 \%(587)$ & $\approx 6 \%$ & \\
\hline \multicolumn{5}{|l|}{ Ethnicity } \\
\hline Hispanic or Latino & $4.6 \%(21)$ & $5.7 \%(49)$ & $\approx 1 \%$ & $X^{2}=0.68579$ \\
\hline Non-Hispanic or Latino & $92.8 \%(425)$ & $91.7 \%(795)$ & $\approx 1 \%$ & $d f=2$ \\
\hline Unknown & $2.6 \%(12)$ & $2.7 \%(23)$ & $\approx<1 \%$ & $p>0.05$ \\
\hline \multicolumn{5}{|l|}{ Gender*** } \\
\hline Male & $91.3 \%(418)$ & $96.0 \%(832)$ & $\approx 5 \%$ & $X^{2}=11.516$ \\
\hline Female & $8.7 \%(40)$ & $4.0 \%(35)$ & $\approx 5 \%$ & $\begin{array}{l}d f=1 \\
p<0.05^{c}\end{array}$ \\
\hline \multicolumn{5}{|l|}{ Comorbidities } \\
\hline Co-Occurring Substance Use Disorder & $58.1 \%(266)$ & $61.7 \%(535)$ & $\approx 3 \%$ & $\begin{array}{l}X^{2}=1.5021 \\
d f=1 \\
p>0.05^{c}\end{array}$ \\
\hline Co-Occurring Mental Health & $74.2 \%(340)$ & $76.4 \%(662)$ & $\approx 2 \%$ & $\begin{array}{l}x^{2}=0.61981 \\
d f=1 \\
p>0.05^{c}\end{array}$ \\
\hline
\end{tabular}


5.3.2. Admission characteristics. The OAT-discontinued and OAT-continued groups differed on two admission-related characteristics: length of stay and receipt of surgical service. Length of stay was likely the only clinically meaningful difference. Discontinued OAT patients had a median stay of three days while OAT-continued patients had a median stay of five days. There were also important similarities between the groups, specifically: 1) the two groups did not differ on source of admission; 2) patients did not differ in acuity of services (e.g., ICU use); and 3) there were no differences in frequency of acute OUD diagnoses and or/OUD-related infections (see Table 5.18). These findings suggest other unmeasured sources were contributing to the differences in care. 
Table 5.18. Admission-Related Patient Characteristics: OAT Continued vs. Discontinued

\begin{tabular}{|c|c|c|c|c|}
\hline Characteristics & $\begin{array}{l}\text { OAT Discontinued } \\
(n=458)\end{array}$ & $\begin{array}{l}\text { OAT Continued } \\
\quad(n=867)\end{array}$ & Difference & $\begin{array}{c}\text { Test } \\
\text { Statistic }\end{array}$ \\
\hline Length of Stay*** & $\begin{array}{l}\text { Median, } 3 \text { days } \\
\text { Mean, } 4.46 \\
\text { (SD 4.84; } 1-43 \text { ) }\end{array}$ & $\begin{array}{l}\text { Median, } 5 \text { days } \\
\text { Mean, } 6.65 \\
\text { (SD 5.88, 1- 47) }\end{array}$ & 2 days & $\begin{array}{l}W=266920 \\
p<0.05^{a}\end{array}$ \\
\hline \multicolumn{5}{|l|}{ Admission Source } \\
\hline Outpatient Treatment & $48.0 \%(220)$ & $45.6 \%(395)$ & $\approx 2 \%$ & $x^{2}=1.6934$ \\
\hline Other Direct Admission & $46.5 \%(213)$ & $49.9 \%(433)$ & $\approx 2 \%$ & $d f=2$ \\
\hline Other & $5.4 \%(25)$ & $4.5 \%(39)$ & $\approx 1 \%$ & $p>0.05^{b}$ \\
\hline \multicolumn{5}{|l|}{ Type of Services Received } \\
\hline ICU Services & $15.9 \%(73)$ & $15.9 \%(138)$ & No diff. & $\begin{array}{l}X^{2}=7.2 \times 10^{-30} \\
d f=1 \\
p=1^{c}\end{array}$ \\
\hline Surgical Services** & $10.3 \%(47)$ & $5.4 \%(47)$ & $\approx 5 \%$ & $\begin{array}{l}X^{2}=9.934 \\
d f=1 \\
p<0.05^{c}\end{array}$ \\
\hline \multicolumn{5}{|l|}{ OUD or Infection Diagnosis } \\
\hline Primary or Secondary OUD Diagnosis & $26.6 \%(122)$ & $27.2 \%(236)$ & $\approx<1 \%$ & $\begin{array}{l}x^{2}=0.026289 \\
d f=1 \\
p>0.05^{c}\end{array}$ \\
\hline OUD Infection Diagnosis & $5.0 \%(23)$ & $6.3 \%(55)$ & $\approx 1 \%$ & $\begin{array}{l}X^{2}=0.72166 \\
d f=1 \\
p>0.05^{c}\end{array}$ \\
\hline OUD Diagnosis or Infection Diagnosis & $31.2 \%(143)$ & $32.9 \%(285)$ & $\approx 2 \%$ & $\begin{array}{l}X^{2}=0.30117 \\
d f=1 \\
p>0.05^{c}\end{array}$ \\
\hline
\end{tabular}


5.3.3. Care delivery during admission. Medications provided during admission differed statistically for patients who discontinued or continued OAT for opioid administration, any adjuvant administration, and second-line adjuvant administration. Of these observed differences, only one of the care delivery processes was likely clinically meaningful: OAT discontinued patients were more likely to receive non-OAT opioids during admission (44\% vs. $26 \%$ ) (see Table 5.19).

Table 5.19. Admission Non-OAT Pharmacotherapy: OAT Continued vs. Discontinued

\begin{tabular}{|c|c|c|c|c|}
\hline Characteristics & $\begin{array}{l}\text { OAT Discontinued } \\
\qquad(n=458)\end{array}$ & $\begin{array}{l}\text { OAT Continued } \\
\qquad(n=867)\end{array}$ & Difference & $\begin{array}{c}\text { Test } \\
\text { Statistic }\end{array}$ \\
\hline Non-OAT Opioid*** & $43.9 \%(201)$ & $26.2 \%(227)$ & $\approx 18 \%$ & $\begin{array}{l}X^{2}=42.15 \\
d f=1 \\
p<0.05^{c}\end{array}$ \\
\hline Any Adjuvant* & $37.3 \%(171)$ & $43.7 \%(379)$ & $\approx 7 \%$ & $\begin{array}{l}X^{2}=4.7615 \\
d f=1 \\
p<0.05^{c}\end{array}$ \\
\hline $\begin{array}{l}\text { First-Line } \\
\text { Adjuvant: Clonidine }\end{array}$ & $10.3 \%(47)$ & $9.7 \%(84)$ & $\approx<1 \%$ & $\begin{array}{l}X^{2}=0.055607 \\
d f=1 \\
p>0.05^{c}\end{array}$ \\
\hline $\begin{array}{l}\text { Second-Line } \\
\text { Adjuvant** }\end{array}$ & $31.2 \%(143)$ & $39.1 \%(339)$ & $\approx 8 \%$ & $\begin{array}{l}X^{2}=7.6988 \\
d f=1 \\
p<0.05^{c}\end{array}$ \\
\hline Benzodiazepine & $23.6 \%(108)$ & $23.5 \%(204)$ & $\approx<1 \%$ & $\begin{array}{l}X^{2}=5.5 \times 10^{-29} \\
d f=1 \\
p=1^{c}\end{array}$ \\
\hline Naltrexone & $<1 \%(2)$ & $<1 \%(2)$ & $\approx<1 \%$ & $\begin{array}{l}X^{2}=0.01527 \\
d f=1 \\
p>0.05^{c}\end{array}$ \\
\hline
\end{tabular}

5.3.4. Outcome characteristics. Compared with the OAT discontinued patients, the OAT continued patients were statistically less likely to leave the hospital against medical advice ( $3 \%$ vs. $10 \%$ ), less likely to receive post-admission opioid prescriptions (12\% vs. $27 \%)$, and were more likely to receive OAT upon discharge in the postadmission period (83\% vs. 64\%) (see Table 5.20). 
Table 5.20. Outcomes: OAT Continued vs. Discontinued

\begin{tabular}{|c|c|c|c|c|}
\hline Characteristics & $\begin{array}{c}\text { OAT } \\
\text { Discontinued } \\
(n=458)\end{array}$ & $\begin{array}{l}\text { OAT Continued } \\
\quad(n=867)\end{array}$ & Difference & $\begin{array}{c}\text { Test } \\
\text { Statistic }\end{array}$ \\
\hline \multicolumn{5}{|l|}{ Admission } \\
\hline In-Hospital Mortality & $<1 \%(1)$ & $1.0 \%(5)$ & $\approx<1 \%$ & $\begin{array}{l}x^{2}=0.24386 \\
d f=1 \\
p>0.05\end{array}$ \\
\hline $\begin{array}{l}\text { Left Against Medical } \\
\text { Advice*** }\end{array}$ & $10.0 \%(46)$ & $3.0 \%(26)$ & $\approx 7 \%$ & $\begin{array}{l}X^{2}=27.589 \\
d f=1 \\
p<0.05\end{array}$ \\
\hline \multicolumn{5}{|l|}{ Post-Admission } \\
\hline Death & $10.0 \%(5)$ & $3.0 \%(7)$ & $\approx 7 \%$ & $\begin{array}{l}X 2=0.046088 \\
d f=1 \\
p>0.05\end{array}$ \\
\hline $\begin{array}{l}\text { Emergency } \\
\text { Department Visit }\end{array}$ & $29.5 \%(135)$ & $26.2 \%(227)$ & $\approx 4 \%$ & $\begin{array}{l}X^{2}=1.4757 \\
d f=1 \\
p>0.05\end{array}$ \\
\hline Hospital Readmission & $12.9 \%(59)$ & $11.8 \%(102)$ & $\approx 1 \%$ & $\begin{array}{l}X^{2}=0.25637 \\
d f=1 \\
p>0.05\end{array}$ \\
\hline Non-OAT Opioid*** & $26.9 \%(123)$ & $12.0 \%(104)$ & $\approx 15 \%$ & $\begin{array}{l}X^{2}=45.576 \\
d f=1 \\
p<0.05\end{array}$ \\
\hline Benzodiazepine & $6.3 \%(29)$ & $4.7 \%(41)$ & $\approx 1 \%$ & $\begin{array}{l}X^{2}=1.2352 \\
d f=1 \\
p>0.05\end{array}$ \\
\hline Naloxone & $8.1 \%(37)$ & $8.7 \%(75)$ & $\approx<1 \%$ & $\begin{array}{l}X^{2}=0.063547 \\
d f=1 \\
p>0.05\end{array}$ \\
\hline Gabapentin/Pregabalin & $28.8 \%(132)$ & $26.3 \%(228)$ & $\approx 2 \%$ & $\begin{array}{l}X^{2}=0.84105 \\
d f=1 \\
p>0.05\end{array}$ \\
\hline Naltrexone & $2.0 \%(9)$ & $<1 \%(6)$ & $\approx 2 \%$ & $\begin{array}{l}X^{2}=3.2764 \\
d f=1 \\
p>0.05\end{array}$ \\
\hline $\begin{array}{l}\text { Concurrent Opioid \& } \\
\text { Benzodiazepine }\end{array}$ & $2.0 \%(9)$ & $1.2 \%(10)$ & $\approx 1 \%$ & $\begin{array}{l}X^{2}=0.88162 \\
d f=1 \\
p>0.05\end{array}$ \\
\hline $\mathrm{OAT}^{* * *}$ & $64.4 \%(295)$ & $83.3 \%(722)$ & $\approx 19 \%$ & $\begin{array}{l}X^{2}=58.272 \\
d f=1 \\
p<0.05\end{array}$ \\
\hline
\end{tabular}


5.3.5. Summary. This section provides insights about a special subset of patients from the study cohort-patients receiving OAT in the 30 days prior to their hospital admission. Several findings stand out. First the difference in OAT receipt during the 30 days after hospital discharge (i.e., the post-admission period) for patients who were either continued (83\%) or discontinued (64\%) on OAT during the admission was significant. This difference illustrates the importance of hospital-based continuation of OAT. Further, this suggests that care pathways or care transition plans may exist for patients in the VHA system who are on OAT prior to admission.

Second, there were differences in patient-and admission-related characteristics. Patients discontinued from OAT had substantially shorter lengths of stay ( 3 days vs. 5 days) suggesting that something may be characteristically different about this patient population, and the primary reason for their hospitalization. The causes of a shorter length of stay cannot be explicitly determined and there are a variety of factors that could explain this difference. One explanation is that shorter stays are related to a higher occurrence of leaving against medical advice (AMA) in the discontinuation population ( $10 \%$ vs. $3 \%)$. Another explanation is that because the patient has a shorter length of stay, due to non-OUD related factors, it is possible that a patient's withdrawal symptoms were not identified. This misidentification could be due to withdrawal masking because non-OAT opioids were administered during admission. OAT discontinued patients were more likely to receive non-OAT opioids (44\% vs. $26 \%$ ). The elevated rate of opioid use could also indicate the converse -- the identification of 
withdrawal and the use of non-FDA approved opioids to manage withdrawal. Shortacting opioids in this patient population were frequently continued post-admission. Patients discontinued from OAT had increased opioid use post-admission ( $27 \%$ vs. $12 \%$ ), suggesting that discontinuing OAT in the hospital setting increases the opportunity for OUD treatment disruption and the propagation of non-evidence-based care.

There were important non-statistically significant findings from this sub-group analysis. First, the two sub-groups did not differ in hospitalization acuity (ICU services) or types of services received (surgery). These are both potential clinical reasons as to why a patient could be discontinued from OAT. Second, there were no differences between the two groups in the frequency of acute OUD diagnoses and or/OUD-related infections. This is a relevant finding because patients with an acute OUD diagnosis may have more obvious needs for OAT continuation. These non-significant findings indicate that the differences in OAT continuation may be explained by other unmeasurable elements, such as the care delivery environment.

\section{Section 5.4. Hospital OAT Delivery Distribution and Performance Quartiles}

To expand upon previous observations that patient and hospital characteristics may contribute to hospital OAT delivery variation, this section further investigates hospital OAT care variation across the VHA system. This section addresses the following research sub-questions:

- 2f: Do outcomes differ for patients who received OAT and for those who did not receive OAT while hospitalized? 
- 2g: Are specific OAT care scenarios associated with hospital OAT delivery quartile?

- 2h: Which hospital attributes associated with hospital OAT delivery?

- $2 \mathrm{i}$ : Are non-OAT pharmacotherapy services associated with hospital OAT delivery quartile?

- $2 \mathrm{j}$. Were outcomes associated with hospital OAT delivery performance?

The relative OAT delivery frequency (the number of admissions with OAT delivered divided by total number of admissions at that facility) was calculated for each facility $(n=109)$. The median relative frequency was $11 \%$, with a standard deviation of 0.10 , and a range from $0 \%$ to $43 \%$, confirming variation in hospital OAT delivery across the VHA system. An assessment of specific OAT delivery mechanisms found that the most common OAT delivery scenarios were withdrawal management (39\%) and OAT sustained (33\%); OAT initiation and linkage to care (9\%) and OAT continuation (7\%) were uncommon (see Table 5.21 and Figure 5.1).

Table 5.21. Hospital OAT Delivery Mechanisms

\begin{tabular}{|c|c|c|c|c|c|c|}
\hline $\begin{array}{l}\text { OAT Delivery } \\
\text { Scenarios }\end{array}$ & $\begin{array}{l}\text { Rel. } \\
\text { Frequency }\end{array}$ & Median & Range & IQR & Skew & Kurtosis \\
\hline $\begin{array}{l}\text { Any OAT } \\
(n=1914)\end{array}$ & $100 \%$ & 0.12 & $0,0.43$ & 0.11 & 1.10 & 0.77 \\
\hline $\begin{array}{l}\text { Withdrawal Management } \\
(\mathrm{n}=844)\end{array}$ & 38.6 & 0.05 & $0.0,0.39$ & 0.06 & 1.32 & 1.99 \\
\hline $\begin{array}{l}\text { OAT Sustained } \\
(\mathrm{n}=722)\end{array}$ & $33.0 \%$ & 0.04 & $0.0,0.23$ & 0.06 & 1.38 & -1.24 \\
\hline $\begin{array}{l}\text { OAT Initiation \& Linkage } \\
(n=203)\end{array}$ & $9.2 \%$ & 0.005 & $0.0,0.08$ & 0.02 & 1.77 & 3.13 \\
\hline $\begin{array}{l}\text { OAT Continued }^{d} \\
(n=145)\end{array}$ & $6.6 \%$ & 0.006 & $0 . .0,0.06$ & 0.02 & 1.38 & 1.37 \\
\hline \multicolumn{7}{|c|}{$\begin{array}{l}\text { Table Notes. }{ }^{a} \text { OAT received during admission period, but not in pre or post period; }{ }^{b} \text { OAT receivea } \\
\text { in the pre, admission, and post periods; }{ }^{c} \text { OAT received during admission and the post period, but } \\
\text { not the pre period; }{ }^{d} O A T \text { received in the pre and admission periods, but not the post period. }\end{array}$} \\
\hline
\end{tabular}




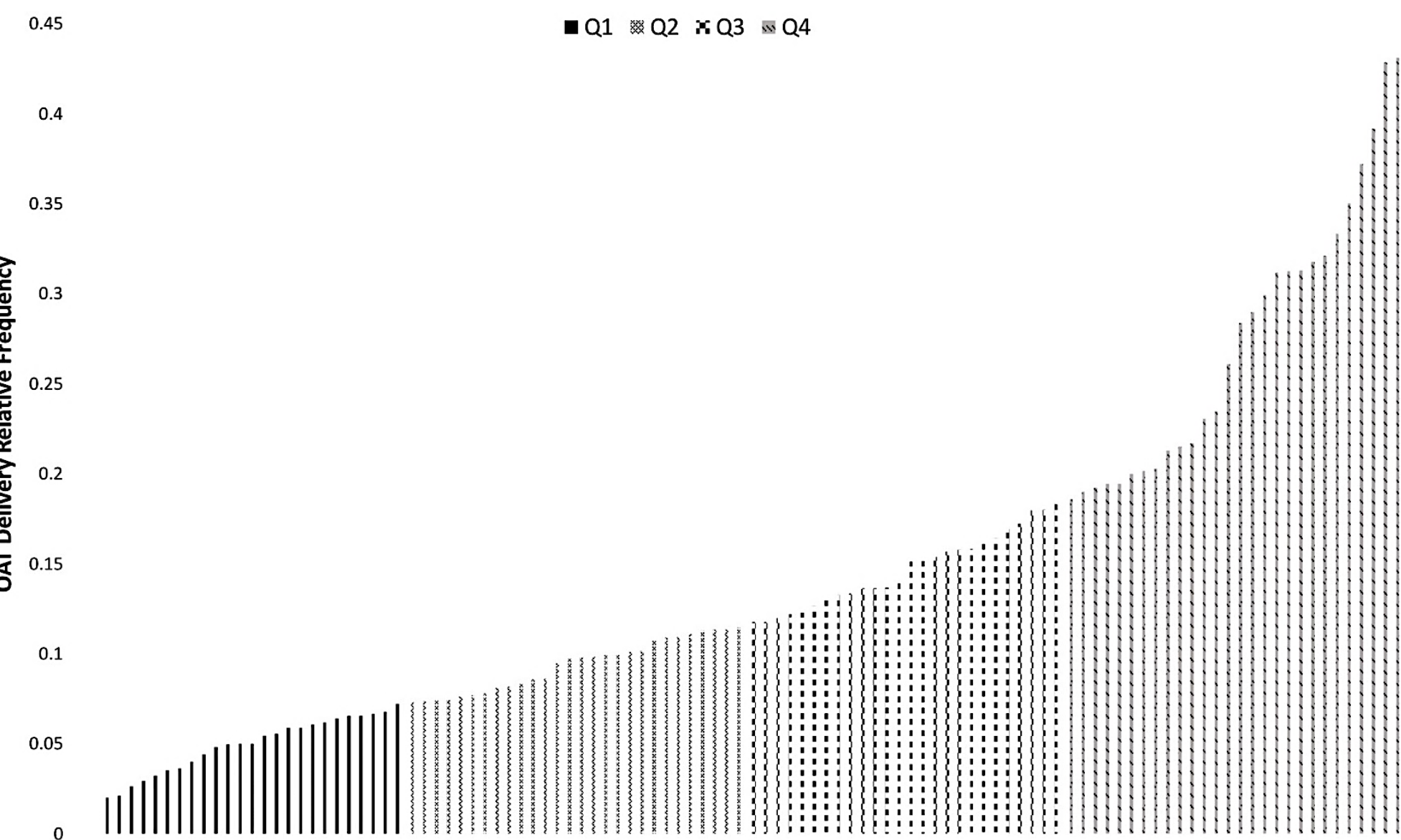

VHA Hospitals $(n=107) *$

Figure 5.1. Relative OAT Frequency for Each Hospital by Quartile

Figure Notes. *Two hospitals had O OAT delivery admissions and are not plotted in the graph. 
Based on the relative OAT delivery frequency (of any OAT delivery scenario), facilities were categorized into hospital OAT delivery performance quartiles: Q1 (0 to 6.0\%), Q2 (7.0 to $11.0 \%)$, Q3 (12.0 to $18.0 \%)$, and Q4 (18.1 to $42.0 \%)$. The data had a non-normal distribution skewed towards lower performance (see Table 5.22 and Figure 5.2.).

Table 5.22. Any OAT Received: By OAT Performance Quartile ${ }^{a}$

\begin{tabular}{lllllll}
\hline $\begin{array}{c}\text { Performance } \\
\text { Quartile }\end{array}$ & $\begin{array}{c}\text { No. of } \\
\text { Obs. }\end{array}$ & Median & Range & IQR & Skew & Kurtosis \\
\hline All $(n=109)$ & 12,407 & 0.12 & $0.0,0.43$ & 0.11 & 1.05 & 0.73 \\
Q4 $(n=28)$ & 3,944 & 0.27 & $0.18,0.43$ & 0.12 & 0.51 & -0.97 \\
Q3 $(n=26)$ & 2,767 & 0.15 & $0.12,0.18$ & 0.03 & 0.21 & -1.28 \\
Q2 $(n=28)$ & 2,836 & 0.10 & $0.07,0.12$ & 0.03 & -0.05 & -1.57 \\
Q1 $(n=27)$ & 2,860 & 0.05 & $0.0,0.07$ & 0.03 & -0.78 & -0.33 \\
\hline
\end{tabular}




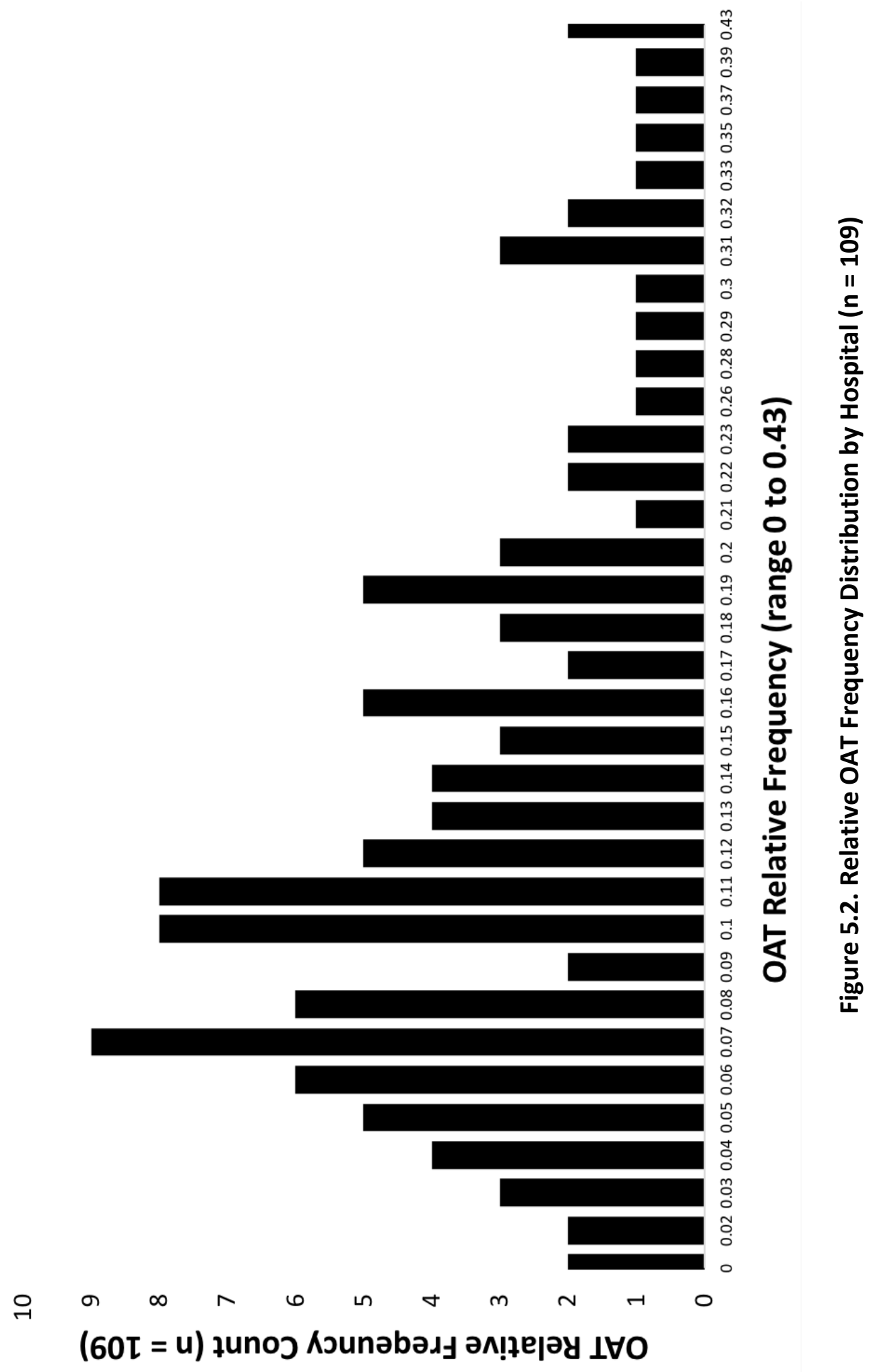


5.4.1. Performance quartiles: OAT delivery. Hospital OAT performance delivery quartiles differed for all OAT care delivery scenarios. For any OAT received, each quartile differed statistically from each other (alpha $=0.05)$, indicating that quartiles provided a meaningful distinction of OAT performance. Generally, facilities in Quartile 4 were more likely to use OAT to manage withdrawal symptoms, to sustain OAT through the care delivery continuum, to continue OAT, and to initiate OAT and link patients to care. The median delivery of OAT in this quartile (27\%), however, suggests that most patients, even in the highest performing hospitals, did not receive OAT. Even the highest perform OAT hospitals in the VHA did not appear to meet current standards of care for addressing OUD (see Table 5.23). 
Table 5.23. OAT Delivery Scenarios by VHA Hospital OAT Delivery Performance Quartile

\begin{tabular}{|c|c|c|c|c|c|c|c|}
\hline $\begin{array}{l}\text { OAT Delivery } \\
\text { Scenario }\end{array}$ & $\begin{array}{c}\text { Proportion, } \\
\text { Median (Range), } \\
\% \\
\text { (n= 109) }\end{array}$ & $\begin{array}{c}\text { Q1 } \\
\text { Proportion, } \\
\text { Median, \% } \\
\text { (n=27) }\end{array}$ & $\begin{array}{c}\text { Q2 } \\
\text { Proportion, } \\
\text { Median, \% } \\
\text { (n=28) }\end{array}$ & $\begin{array}{c}\text { Q3 } \\
\text { Proportion, } \\
\text { Median, \% } \\
(n=26)\end{array}$ & $\begin{array}{c}\text { Q4 } \\
\text { Proportion, } \\
\text { Median, \% } \\
(n=28)\end{array}$ & $\begin{array}{c}\text { Q4-Q1 } \\
\text { Diff }\end{array}$ & $\begin{array}{c}\text { Test } \\
\text { Statistic }^{b}\end{array}$ \\
\hline OAT Received*** & $\begin{array}{c}12 \% \\
(0.0-0.43)\end{array}$ & $5.0 \%$ d,e,f & $9.8 \%^{d, g, i}$ & $14.5 \% \%^{\mathrm{e}, g, h}$ & $27.2 \%^{f, h, i}$ & $\approx 22 \%$ & $\begin{array}{l}X^{2}=101.24 \\
d f=3 \\
p<0.05^{c}\end{array}$ \\
\hline $\begin{array}{l}\text { Withdrawal } \\
\text { Management*** }\end{array}$ & $\begin{array}{c}5 \% \\
(0.0-0.39)\end{array}$ & $2.5 \%$ d,e,f & $5.1 \% \mathrm{~d}, \mathrm{i}$ & $7.0 \%$ eh & $11.0 \%$ f,h,i & $\approx 8 \%$ & $\begin{array}{l}X^{2}=53.051 \\
d f=3 \\
p<0.05^{c}\end{array}$ \\
\hline OAT Sustained*** & $\begin{array}{c}4 \% \\
(0.0-0.23)\end{array}$ & $1.4 \%^{\mathrm{d}, \mathrm{e}, \mathrm{f}}$ & $2.6 \%$ d,i & $4.0 \% \mathrm{e}^{\mathrm{h}}$ & $9.6 \%$, ,h,i & $\approx 8 \%$ & $\begin{array}{l}X^{2}=54.929 \\
d f=3 \\
p<0.05^{c}\end{array}$ \\
\hline $\begin{array}{l}\text { OAT } \\
\text { Continued*** }\end{array}$ & $\begin{array}{c}<1 \% \\
(0.0-0.06)\end{array}$ & $0 \%$ e,f & $<1 \%$ & $<1 \% \mathrm{e}^{\mathrm{e}}$ & $1.6 \%^{\mathrm{f}, \mathrm{i}}$ & $\approx 2 \%$ & $\begin{array}{l}X^{2}=18.467 \\
d f=3 \\
p<0.05^{c}\end{array}$ \\
\hline $\begin{array}{l}\text { OAT Initiation and } \\
\text { Linkage*** }\end{array}$ & $\begin{array}{c}<1 \% \\
(0.0-0.08)\end{array}$ & $0 \%$ e,f & $0 \% \%^{\mathrm{si}}$ & $1.4 \% \mathrm{e}, \mathrm{g}$ & $2.5 \%$ f,i & $\approx 3 \%$ & $\begin{array}{l}X^{2}=38.2776 \\
d f=3 \\
p<0.05^{c}\end{array}$ \\
\hline \multicolumn{8}{|c|}{$\begin{array}{l}\text { Table Notes. }{ }^{a} \text { Hospitals were categorized into OAT delivery performance quartiles based on the proportion of cases at a hospital with OAT } \\
\text { during admission. Q1 hospitals were the lowest performers and Q4 the highest; }{ }^{b} \text { Kruskal-Wallis Test; 'Dunn's Test; 'Dunn's Test results: } \\
\text { difference between Q1 and Q2; }{ }^{e} \text { Dunn's Test results: difference between Q1 and Q3; }{ }^{f} \text { Dunn's Test results: difference between Q1 and Q4; } \\
{ }^{g} \text { Dunn's Test results: difference between Q2 and Q3; }{ }^{h} \text { Dunn's Test results: difference between Q3 and Q4; 'Dunn's Test results: difference } \\
\text { between Q2 and Q4; } p<0.05^{*} ; p<0.01^{* * ;} p<0.001^{* * *} \text {. }\end{array}$} \\
\hline
\end{tabular}


5.4.2. Performance quartiles: Hospital characteristics. Next, relationships among hospital OAT performance quartile and supply-side characteristics (i.e., size and region) were assessed. OAT performance delivery quartiles differed on the number of hospital beds and the geographic location of the facility, but not for hospital volume of acute OUD diagnoses and/or OUD-related infections. Meaningful differences appeared to exist between Quartile 4 (highest performer) and Quartile 1 (lowest performer). Quartile 4 included fewer small hospitals (4\% vs. $26 \%$ ), more large hospitals (57\% vs. $37 \%)$, and a different geographic distribution of facilities. Quartile 4 included more hospitals in the Midwest (25\% vs. $15 \%)$, Northeast (32\% vs. $7 \%)$, and the West (21\% vs. $4 \%)$, and fewer hospitals in the South (21\% vs. $74 \%$ ) (see Table 5.24$)$. 
Table 5.24. Hospital Characteristics by VHA Hospital OAT Delivery Performance Quartile ${ }^{a, b}$

\begin{tabular}{|c|c|c|c|c|c|c|c|}
\hline Characteristics & $\begin{array}{c}\text { All } \\
(n=109)\end{array}$ & $\begin{array}{l}\text { Quartile } 1 \\
(n=27)\end{array}$ & $\begin{array}{l}\text { Quartile } 2 \\
(n=28)\end{array}$ & $\begin{array}{c}\text { Quartile } 3 \\
(n=26)\end{array}$ & $\begin{array}{c}\text { Quartile } 4 \\
(n=28)\end{array}$ & $\begin{array}{c}\text { Q4-Q1 } \\
\text { Diff }\end{array}$ & $\begin{array}{c}\text { Test } \\
\text { Statistic }\end{array}$ \\
\hline $\begin{array}{l}\text { Acute OUD } \\
\text { Diagnosis Volume }\end{array}$ & $\begin{array}{l}\text { Median, } 0.32 \\
\text { Range } \\
0.13,0.83\end{array}$ & $\begin{array}{l}\text { Median, } 0.34 \\
\text { Range } \\
0.22,0.56\end{array}$ & $\begin{array}{l}\text { Median, } 0.33 \\
\text { Range } \\
0.21,0.55\end{array}$ & $\begin{array}{l}\text { Median, } 0.29 \\
\text { Range } \\
0.18,0.45\end{array}$ & $\begin{array}{l}\text { Median, } 0.28 \\
\text { Range } \\
0.13,0.83\end{array}$ & 0.6 & $\begin{array}{l}X^{2}=3.818 \\
d f=3 \\
p>0.05^{d}\end{array}$ \\
\hline Admission Volume & $\begin{array}{l}\text { Median, } 98 \\
\text { Range } \\
26,430 \\
\end{array}$ & $\begin{array}{l}\text { Median, } 78 \\
\text { Range } \\
33,430 \\
\end{array}$ & $\begin{array}{l}\text { Median, } 97.5 \\
\text { Range } \\
26,221 \\
\end{array}$ & $\begin{array}{l}\text { Median, } 104 \\
\text { Range } \\
30,213 \\
\end{array}$ & $\begin{array}{l}\text { Median, } 136 \\
\text { Range } \\
35,294 \\
\end{array}$ & 58 & $\begin{array}{l}X^{2}=5.206 \\
d f=3 \\
p>0.05^{d}\end{array}$ \\
\hline $\begin{array}{l}\text { Hospital Size* } \\
\text { Small } \\
\text { Medium } \\
\text { Large }\end{array}$ & $\begin{array}{l}25.7 \%(28) \\
33.0 \%(36) \\
41.3 \%(45) \\
\end{array}$ & $\begin{array}{c}25.9 \%(7) \\
37.0 \%(10) \\
37.0 \%(10)\end{array}$ & $\begin{array}{c}39.3 \%(11) \\
25.0 \%(7) \\
35.7 \%(10)\end{array}$ & $\begin{array}{c}23.1 \%(6) \\
30.8(8) \\
46.2 \%(12) \\
\end{array}$ & $\begin{array}{c}3.6 \%(1) \\
39.3 \%(11) \\
57.1 \%(16) \\
\end{array}$ & $\begin{array}{l}\approx 22 \% \\
\approx 2 \% \\
\approx 20 \%\end{array}$ & $p<0.05^{c}$ \\
\hline $\begin{array}{l}\text { U.S. Region** } \\
\text { Midwest } \\
\text { Northeast } \\
\text { South } \\
\text { West }\end{array}$ & $\begin{array}{l}22.9 \%(25) \\
16.5 \%(18) \\
39.4 \%(43) \\
21.1 \%(23)\end{array}$ & $\begin{array}{c}14.8 \%(4) \\
7.4 \%(2) \\
74.1 \%(20) \\
3.7 \%(1)\end{array}$ & $\begin{array}{l}28.6 \%(8) \\
10.7 \%(3) \\
25.0 \%(7) \\
35.7 \%(10)\end{array}$ & $\begin{array}{c}23.1 \%(6) \\
15.4 \%(4) \\
38.5 \%(10) \\
23.1 \%(6)\end{array}$ & $\begin{array}{l}25.0 \%(7) \\
32.1 \%(9) \\
21.4 \%(6) \\
21.4 \%(6)\end{array}$ & $\begin{array}{l}\approx 10 \% \\
\approx 25 \% \\
\approx 52 \% \\
\approx 17 \%\end{array}$ & $p<0.05^{c}$ \\
\hline $\begin{array}{l}\text { Table Notes }{ }^{a} \text { Hospit } \\
\text { admission. Q1 hosp } \\
\text { because some of th } \\
\text { Large hospital }=>9\end{array}$ & $\begin{array}{l}\text { s were catego } \\
\text { als were the lc } \\
\text { cell values we } \\
\text { beds; } p<0.05\end{array}$ & $\begin{array}{l}\text { to OAT delive } \\
\text { erformers an } \\
\text { han } 5 ;{ }^{d} \text { Krusk } \\
.01^{* *} ; p<0 .\end{array}$ & $\begin{array}{l}\text { rmance quar } \\
\text { highest; }{ }^{b} Q u \\
\text { is Rank Sum T }\end{array}$ & $\begin{array}{l}\text { sed on the pr } \\
\text { ercentages W } \\
\text { lall hospital = }\end{array}$ & $\begin{array}{l}\text { of cases at a } \\
\text { llated by row, } \\
\text { ds; Medium h }\end{array}$ & $\begin{array}{l}\text { tal with } \\
\text { er's Exac } \\
l=50-9\end{array}$ & $\begin{array}{l}\text { during } \\
\text { t was used } \\
\text { s; }\end{array}$ \\
\hline
\end{tabular}




\subsubsection{Performance quartiles: Non-OAT pharmacotherapy. OAT performance}

quartiles differed statistically for the delivery of non-OAT opioids, any withdrawal management adjuvant, and second-line adjuvant. Quartile 4 facilities were significantly less likely to administer opioids compared with the other OAT performance quartiles (Q1, Q2, and Q3). Quartile 4 hospitals also had lower use of adjuvant and second-line adjuvant administration versus the bottom quartile (Q1). There were no statistically significant differences among quartiles for use of benzodiazepines, which across the study population was relatively high (median - 32\%; range $13 \%$ to $83 \%$ of admissions), and for naltrexone which across the study population was relatively low (median $<1 \%$; range $0 \%$ to $8 \%$ ) (see Table 5.25 ). 
Table 5.25. Non-OAT Pharmacotherapy Delivery by VHA Hospital OAT Delivery Performance Quartile ${ }^{\mathrm{a}}$

\begin{tabular}{|c|c|c|c|c|c|c|c|}
\hline $\begin{array}{c}\text { Care } \\
\text { Delivery } \\
\text { Scenario }\end{array}$ & $\begin{array}{l}\text { Prop., } \\
\text { Median \% } \\
\text { (Range) }\end{array}$ & $\begin{array}{c}\text { Q1 } \\
(n=27) \\
\text { Prop., Median \% }\end{array}$ & $\begin{array}{c}\text { Q2 } \\
(n=28) \\
\text { Prop., Median \% }\end{array}$ & $\begin{array}{c}\text { Q3 } \\
(n=26) \\
\text { Prop., Median \% }\end{array}$ & $\begin{array}{c}\text { Q4 } \\
(n=28) \\
\text { Prop., Median \% }\end{array}$ & $\begin{array}{c}\text { Q4-Q1 } \\
\text { Diff }\end{array}$ & $\begin{array}{c}\text { Test } \\
\text { Statistic }^{b}\end{array}$ \\
\hline Opioid** & $\begin{array}{c}53.8 \% \\
(0.2,0.83)\end{array}$ & $56.5 \%^{f}$ & $59.3 \%^{i}$ & $54.1 \%^{\mathrm{h}}$ & $46.8 \%^{f, h, i}$ & $\approx 10 \%$ & $\begin{array}{l}X^{2}=15.556 \\
d f=3 \\
p<0.05^{c}\end{array}$ \\
\hline $\begin{array}{l}\text { Any } \\
\text { Adjuvant** }\end{array}$ & $\begin{array}{c}44.4 \% \\
(0.21,0.74)\end{array}$ & $48.5 \%$ e,f & $45.2 \%$ & $42.9 \% \mathrm{e}^{-}$ & $41.6 \%^{\mathrm{f}}$ & $\approx 6 \%$ & $\begin{array}{l}X^{2}=12.085 \\
d f=3 \\
p<0.05^{c}\end{array}$ \\
\hline First-Line $^{1}$ & $\begin{array}{c}7.7 \% \\
(0.0,0.40)\end{array}$ & $7.7 \%$ & $7.7 \%$ & $8.2 \%$ & $6.9 \%$ & $\approx<1 \%$ & $\begin{array}{l}X^{2}= \\
0.21976 \\
d f=3 \\
p>0.05\end{array}$ \\
\hline $\begin{array}{l}\text { Second- } \\
\text { Line } 2 * *\end{array}$ & $\begin{array}{c}39.0 \% \\
(0.16,0.69)\end{array}$ & $44.7 \%$, f & $41.4 \%$ & $36.2 \% \mathrm{e}$ & $36.8 \%^{f}$ & $\approx 8 \%$ & $\begin{array}{l}X^{2}=16.12 \\
d f=3 \\
p<0.05^{c}\end{array}$ \\
\hline $\begin{array}{l}\text { Benzo- } \\
\text { diazepine }\end{array}$ & $\begin{array}{c}32.2 \% \\
(0.13,0.83)\end{array}$ & $34.0 \%$ & $33.2 \%$ & $29.3 \%$ & $28.2 \%$ & $\approx 6 \%$ & $\begin{array}{l}X^{2}=0.2818 \\
d f=3 \\
p>0.05^{c}\end{array}$ \\
\hline Naltrexone & $\begin{array}{c}<1 \% \\
(0.0,0.08)\end{array}$ & $1.2 \%$ & $<1 \%$ & $1.2 \%$ & $1.1 \%$ & $\approx<1 \%$ & $\begin{array}{l}X^{2}=5.1653 \\
d f=3 \\
p>0.05\end{array}$ \\
\hline \multicolumn{8}{|c|}{ 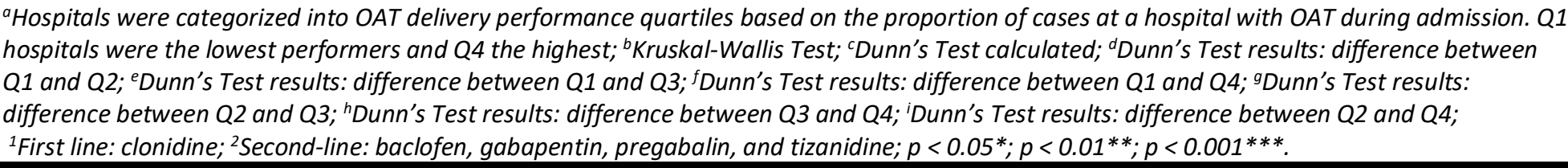 } \\
\hline
\end{tabular}


5.4.4. Performance quartiles: Outcomes. Of the ten health care utilization measures, three differed statistically by OAT quartile. Opioid prescriptions in the 30 days post-admission were less common in Quartile $4(28 \%)$ as compared to the other quartiles (Q1, 37\%; Q2, 41\%; Q3, 34\%). Gabapentin/pregabalin prescriptions in the postadmission period were higher in Quartile $1(29 \%)$ versus Quartile $3(25 \%)$ and Quartile 4 (24\%). Post-admission OAT receipt differed among each of the quartiles: Q1, 4\%; Q2, 7\%; Q3, 10\%; and Q4, 19\%. The frequency of post-admission emergency department visits (median, $29 \%$; range: $0 \%$ to $50 \%$ ) was noteworthy but did not differ significantly. Also, the two mortality measures (in-hospital mortality and post-admission death) were uncommon across all quartiles (see Table 5.26). 
Table 5.26. Outcomes by VHA Hospital OAT Delivery Performance Quartile

\begin{tabular}{|c|c|c|c|c|c|c|c|}
\hline $\begin{array}{l}\text { Outcome } \\
\text { Variable }\end{array}$ & $\begin{array}{l}\text { Prop. } \\
\text { Median } \\
\text { (Range) }\end{array}$ & $\begin{array}{c}\text { Q1 }^{1} \\
(n=27)\end{array}$ & $\begin{array}{c}\text { Q2 }^{1} \\
(n=28)\end{array}$ & $\begin{array}{c}\text { Q3 }^{1} \\
(n=26)\end{array}$ & $\begin{array}{c}\mathrm{Q}^{1} \\
(\mathrm{n}=28)\end{array}$ & $\begin{array}{l}\text { Q4- } \\
\text { Q1 } \\
\text { Diff. }\end{array}$ & $\begin{array}{c}\text { Test } \\
\text { Statistic }^{\mathbf{b}}\end{array}$ \\
\hline \multicolumn{8}{|l|}{ Admission } \\
\hline $\begin{array}{l}\text { In-Hospital } \\
\text { Mortality }\end{array}$ & $\begin{array}{l}<1 \% \\
(0.0-0.05)\end{array}$ & $<1 \%$ & $<1 \%$ & $<1 \%$ & $1.1 \%$ & $\approx<1 \%$ & $\begin{array}{l}X^{2}=2.1073 \\
d f=3 \\
p>0.05\end{array}$ \\
\hline $\begin{array}{l}\text { Left Against } \\
\text { Medical } \\
\text { Advice }\end{array}$ & $\begin{array}{l}5.2 \% \\
(0.0-0.26)\end{array}$ & $5.3 \%$ & $5.7 \%$ & $5.3 \%$ & $5.1 \%$ & $\approx<1 \%$ & $\begin{array}{l}X^{2}=0.31675 \\
d f=3 \\
p>0.05\end{array}$ \\
\hline \multicolumn{8}{|c|}{ Post-Admission } \\
\hline Death & $\begin{array}{l}0 \% \\
(0.0-0.05)\end{array}$ & $1.2 \%$ & $<1 \%$ & $<1 \%$ & $<1 \%$ & $\approx<1 \%$ & $\begin{array}{l}X^{2}=0.83801 \\
d f=3 \\
p>0.05\end{array}$ \\
\hline $\begin{array}{l}\text { Emergency } \\
\text { Department } \\
\text { Visit }\end{array}$ & $\begin{array}{l}28.6 \% \\
(0.0-0.53)\end{array}$ & $25.9 \%$ & $29.6 \%$ & $29.9 \%$ & $27.0 \%$ & $\approx 1 \%$ & $\begin{array}{l}X^{2}=1.5277 \\
d f=3 \\
p>0.05\end{array}$ \\
\hline $\begin{array}{l}\text { Hospital } \\
\text { Readmission }\end{array}$ & $\begin{array}{l}13.1 \% \\
(0.02-0.27)\end{array}$ & $12.0 \%$ & $13.2 \%$ & $12.8 \%$ & $13.8 \%$ & $\approx 2 \%$ & $\begin{array}{l}X^{2}=1.1666 \\
d f=3 \\
p>0.05\end{array}$ \\
\hline Opioid*** & $\begin{array}{l}36.4 \% \\
(0.1-0.69)\end{array}$ & $36.6 \%^{f}$ & $41.2 \%$ & $34.0 \%{ }^{\mathrm{h}}$ & $28.2 \%$ & $\approx 8 \%$ & $\begin{array}{l}X^{2}=16.707 \\
d f=3 \\
p<0.05^{c}\end{array}$ \\
\hline $\begin{array}{l}\text { Benzo- } \\
\text { diazepine }\end{array}$ & $\begin{array}{l}7.8 \% \\
(0.0-0.22)\end{array}$ & $7.1 \%$ & $8.8 \%$ & $7.3 \%$ & $7.6 \%$ & $\approx<1 \%$ & $\begin{array}{l}X^{2}=5.9958 \\
d f=3 \\
p>0.05\end{array}$ \\
\hline Naloxone & $\begin{array}{l}5.0 \% \\
(0.0-0.31)\end{array}$ & $6.4 \%$ & $4.1 \%$ & $5.3 \%$ & $6.8 \%$ & $\approx<1 \%$ & $\begin{array}{l}X^{2}=4.2447 \\
d f=3 \\
p>0.05\end{array}$ \\
\hline $\begin{array}{l}\text { Gabapentin/ } \\
\text { Pregabalin* }\end{array}$ & $\begin{array}{l}26.2 \% \\
(0.13-0.47)\end{array}$ & $28.2 \%$ & $26.2 \%$ & $24.5 \%^{\mathrm{e}}$ & $23.9 \%^{f}$ & $\approx 4 \%$ & $\begin{array}{l}X^{2}=10.161 \\
d f=3 \\
p<0.05^{c}\end{array}$ \\
\hline Naltrexone & $\begin{array}{l}<1 \% \\
(0.0-0.08)\end{array}$ & $1.2 \%$ & $<1 \%$ & $1.2 \%$ & $1.1 \%$ & $\approx<1 \%$ & $\begin{array}{l}X^{2}=5.1653 \\
d f=3 \\
p>0.05\end{array}$ \\
\hline $\begin{array}{l}\text { Concurrent } \\
\text { Opioid/ } \\
\text { Benzo- } \\
\text { diazepine }\end{array}$ & $\begin{array}{l}3.9 \% \\
(0.0-0.14)\end{array}$ & $4.0 \%$ & $4.2 \%$ & $3.9 \%$ & $3.4 \%$ & $\approx<1 \%$ & $\begin{array}{l}X^{2}=3.3905 \\
d f=3 \\
p>0.05\end{array}$ \\
\hline $\mathrm{OAT}^{* * *}$ & $\begin{array}{l}8.4 \% \\
(0.0-0.38)\end{array}$ & $3.5 \%$ d,e,f & $6.8 \% \mathrm{~d}, \mathrm{~g}, \mathrm{i}$ & $10.3 \% \mathrm{e}^{\mathrm{e}, \mathrm{g}, \mathrm{h}}$ & $18.5 \%$, h,i & $\approx 15 \%$ & $\begin{array}{l}X^{2}=55.252 \\
d f=3 \\
p<0.05^{c}\end{array}$ \\
\hline \multicolumn{8}{|c|}{ 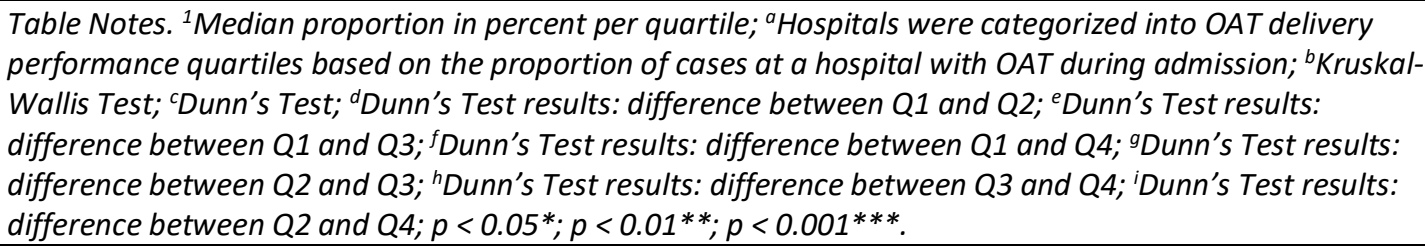 } \\
\hline
\end{tabular}


5.4.5. Summary. OAT delivery in the hospital setting varied across the VHA system, with statistically significant differences observed across hospital performance quartiles. Underperformance across the system was consistent. The highest performing hospitals, in Quartile 4, provided OAT to less than half of their patients with a current or prior OUD diagnosis. The most common OAT delivery scenarios across the VHA system were withdrawal management and sustaining OAT through the VHA care continuum. Hospital OAT performance quartiles differed statistically by the number of hospital beds and the geographic location of the facility, but not for hospital volume of acute OUD diagnoses or OUD-related infections. Hospitals also differed among quartile performance for the delivery of non-OAT opioids, withdrawal management adjuvants, and second-line adjuvants. Finally, of the ten outcome measures, only three differed statistically by OAT quartile: 30-day opioid prescription fills (lowest in Q4), 30-day gabapentin/pregabalin prescription fills (highest in Q1 and lowest in Q4), and 30-day OAT receipt (highest in Q4 and lowest in Q1). The remaining seven health care utilization outcome measures did not differ by quartile. These preliminary and exploratory analyses suggest that contextual attributes within the hospital or the local environment influence OAT and non-OAT care delivery performance and subsequent outcomes for discharged patients.

\section{Section 5.5. Aim 3 Justification}

The findings from Aim 2 support continuing to explore these phenomena through additional contextual analyses in Aim 3. Aim 3 tests associations between 
multilevel attributes and the delivery of OAT during admission for patients hospitalized with OUD-related diagnoses in VHA hospitals.

Aim 2 analyses suggested potentially problematic care patterns for patients with OUD across the VHA system, specifically, that patients frequently received opioids and gabapentin/pregabalin, and not OAT. In all three time periods (pre, admission, post), opioid prescription or administration frequency was higher than OAT delivery. Withdrawal management was the predominant OAT delivery mechanism, which is not the most effective way to treat OUD and not the current standard of care. Additionally, although $21^{\text {st }}$ century harm reduction practices recommend co-prescribing of naloxone with opioid prescriptions or for patients with OUD, few VHA patients received naloxone. It is possible that patients had already been prescribed naloxone 31 days prior to admission and thus already had naloxone in their possession.

Although the study cohort was homogenous for many demand-side attributes (i.e., patient characteristics), the clinical reasons for hospital admission varied. Most admissions were not OUD related (by primary or secondary diagnosis or by OUD-related infection codes). A comparison of those who received and did not receive OAT during admission found that more patients who received OAT had an acute OUD diagnosis and OUD-related infections. However, overall, $60 \%$ of patients who received OAT did not have a primary or secondary OUD diagnosis or an OUD-related infection suggesting unexplained influencers may contribute to OAT delivery during admission. 
Finally, a wide range of OAT administration during hospitalization persistedsome hospitals delivered no OAT $(n=2)$ and some hospitals delivered OAT to $43 \%$ of their patients with OUD $(n=2)$. The frequency of the type of OAT mechanism varied across hospital OAT performance quartile, suggesting that measured (hospital size, geographic location, and hospital OAT performance quartile) and unmeasured supplyside attributes influenced OAT care delivery and performance. Observed differences existed for hospital size, geographic location, and OAT performance quartile, but not for acute OUD case volume. Other elements may drive care delivery variation. Differences existed among hospital OAT performance quartiles for non-OAT care delivery mechanisms, specifically, opioid delivery during and in the 30 days after hospital discharge. Although statistical differences did not exist for most outcomes, one nonsignificant finding stands out: nearly one third of patients received services in the emergency department within 30 days of hospitalization which suggests unresolved care issues post-hospitalization for this patient population.

The Aim 2 descriptive and bivariate analyses justify the continued exploration and understanding of OAT delivery for exploration of Aim 3. Proceeding with Aim 3 is warranted because the current care delivery practices were observed to be sub-optimal, demand-side (patient) and supply-side (hospital) attributes were associated with the receipt of OAT, and hospital OAT delivery varied significantly across the VHA system. Aim 3 explores the unexplained differences among those who receive and do not 
receive OAT when hospitalized in the VHA system and the contributions of patient and hospital-level attributes to OAT delivery using multilevel models.

\section{Section 5.6. Multilevel Model Output}

This section explores the associations of patient and hospital attributes on the receipt of OAT during hospitalization through multilevel modeling. The multilevel model uses the same study cohort as Aim 2, with 12,407 unique index hospitalizations from 109 VHA hospitals within the continental U.S. during the 2017 fiscal year. This section addressed research sub-questions:

- 3a: How much of the variation in OAT delivery is attributable to the hospitals?

- 3b: How do level 1 and level 2 attributes affect hospital OAT delivery?

5.6.4. Multilevel logistic regression model. A multilevel logistic regression model was used to test for associations at the 0.05 alpha value in this study cohort. A patient hospitalized in an average VHA hospital, without controlling for patient-level or hospitallevel covariates, had a $12 \%$ chance of OAT administration. ${ }^{4}$

5.6.4a. Testing for hospital variation. The first model, Model 1, was the variance component model. This model did not include any covariates and only included the intercept. The intraclass correlation statistic (ICC) for Model 1 was 0.14 , meaning that approximately $14 \%$ of the variability of receiving OAT during admission was explained by

\footnotetext{
${ }^{4}$ The probability of receiving OAT in an 'average' VHA facility were estimated as exp(-1.97) = 0.139 with the calculated probability of $0.14 /(1+0.14)=0.12$.
} 
between-hospital differences (e.g., hospital level characteristics). Subsequently, withinhospital differences (e.g., patient level characteristics) explained the other $86 \%$ of the variability for receiving OAT during admission (see Table 5.27).

The second model, Model 2, was the level 1 intermediate model. This model included the intercept and the patient covariates. In this model, there was a decrease in the ICC from Model 1, going from 0.14 to 0.10 , suggesting that some of the variation in OAT delivery across the study cohort may be due to the differences in patient population characteristics among hospitals (e.g., differences in average age or gender distribution). In this model, level 1 covariates collectively accounted for $4 \%$ of OAT delivery variation (see Table 5.27).

The third model, Model 3, was the final model, which included the intercept, patient characteristics, and hospital covariates. When compared with Model 2 , the ICC statistic in this Model decreased from 0.10 to less than 0.06 , respectively, indicating that the inclusion of the available and selected hospital level variables, in part, explained variation between hospitals. However, with an additional $6 \%$ still unexplained by the model, this suggests that additional unexplored or unmeasured level 2 covariates may further explain variation hospital OAT delivery in the VHA system (see Table 5.27).

Table 5.27. Testing for Variation in Hospital OAT Delivery Across the VHA

\begin{tabular}{|c|c|c|c|c|c|c|c|c|c|}
\hline & \multicolumn{3}{|c|}{$\begin{array}{l}\text { Model 1: Without } \\
\text { Covariates }\end{array}$} & \multicolumn{3}{|c|}{$\begin{array}{l}\text { Model 2: Patient } \\
\text { Covariates }\end{array}$} & \multicolumn{3}{|c|}{$\begin{array}{l}\text { Model 3: Patient and } \\
\text { Hospital Covariates }\end{array}$} \\
\hline & $B$ & SE & $95 \% \mathrm{Cl}$ & $B$ & $S E$ & $95 \% \mathrm{Cl}$ & $B$ & $S E$ & $95 \% \mathrm{Cl}$ \\
\hline Constant & 0.52 & 0.09 & $0.37,0.72$ & 0.38 & 0.07 & $0.26,0.56$ & 0.02 & 0.05 & $0.13,0.34$ \\
\hline ICC & 0.14 & 0.02 & $0.10,0.18$ & 0.10 & 0.18 & $0.07,0.14$ & 0.06 & 0.13 & $0.04,0.09$ \\
\hline
\end{tabular}


5.6.4b. Testing for associations among covariates and OAT delivery. In Model 2, the model with only patient characteristics, 12 covariates were associated with either increased or decreased odds of OAT delivery during admission. Six covariates increased the odds of OAT delivery, while holding the other level 1 covariates constant: 1) preadmission OAT receipt (OR [Odds Ratio] 15.3; 95\% Cl $[13.2,17.8]$ ); 2) the presence of an OUD diagnosis or infection (OR 2.30; 95\% Cl [1.99, 2.67]); 3) male gender (OR 1.52; 95\% $\mathrm{Cl}[1.15,2.00]) ; 4)$ an opioid withdrawal diagnosis (OR 1.46; 95\% Cl $[1.12,1.91]) ; 5)$ admission adjuvant receipt (OR 1.53; 95\% $\mathrm{Cl}[1.33,1.76])$; and 6) an increased length of stay (OR 1.04; 95\% Cl [1.04, 1.05]) (see Table 5.28).

Six covariates were associated with decreased odds of OAT delivery during hospitalization, while holding the other level 1 covariates constant: 1) pre-admission naltrexone receipt (OR 0.27; 95\% $\mathrm{Cl}[0.13,0.57]) ; 2)$ unintentional overdose diagnosis (OR 0.29; 95\% Cl $[0.16,0.52]$ ); 3) admission naltrexone receipt (OR 0.30; 95\% Cl [0.14, $0.65]$ ); 4) pre-admission non-OAT opioid receipt (OR 0.49; $95 \% \mathrm{Cl}[0.41,0.58]$ ); 5) admission non-OAT opioid receipt (OR 0.53; $95 \mathrm{Cl}[0.46,0.61])$; and 6) co-occurring SUD diagnosis (OR 0.77; 95\% Cl [0.67, 0.88]) (see Table 5.28).

In Model 3, which included both patient and hospital characteristics, the 12 patient covariates associated with either increased or decreased odds of hospitalized OAT delivery in Model 2 did not qualitatively change with regard to direction or statistical significance. One hospital covariate decreased the odds of OAT delivery slightly, increased hospital acute diagnosis relative volume (OR 0.98; 95\% Cl [0.97, 
0.99]). Four of the hospital-level covariates increased the odds of OAT delivery: size and location. The medium (OR 1.90; 95\% Cl $[1.33,2.70$ ) and large sized hospitals (OR 2.04; $95 \% \mathrm{Cl}[1.39,3.00])$ had increased odds of OAT delivery during admission compared to small hospitals. Hospitals located in the Northeast (OR 1.80; $95 \% \mathrm{Cl}[1.30,2.49])$ and West (OR 1.62; 95\% Cl $[1.19,2.22])$ had increased odds of OAT receipt compared to hospitals located in the South (see Table 5.28).

5.6.4c. OAT sensitivity analysis definition. A sensitivity analysis examined if there were differences in the study results if the definition of hospital OAT delivery was narrowed (Definition 2). The two definitions were: 1) Definition 1: Any type of OAT regardless of FDA-approval of that formulation (e.g., intramuscular/intravenous methadone or buprenorphine); 2) Definition 2: Only FDA-approved formulations of OAT (e.g. oral/sublingual methadone and buprenorphine). When comparing results of the two models, there was only one difference observed for one of the 18 statistically significant covariates. In the model built with Definition 2, the narrower OAT definition, the variable surgical services (a patient covariate) was no longer statistically significant. Otherwise, there were no observed differences in the statistical significance of any of the other 17 covariates or the directionality of the parameters. This suggests that the findings from Aim 1 that non-FDA approved versions of buprenorphine/methadone are used in the hospital setting, may be true. See Appendix R for additional details. 


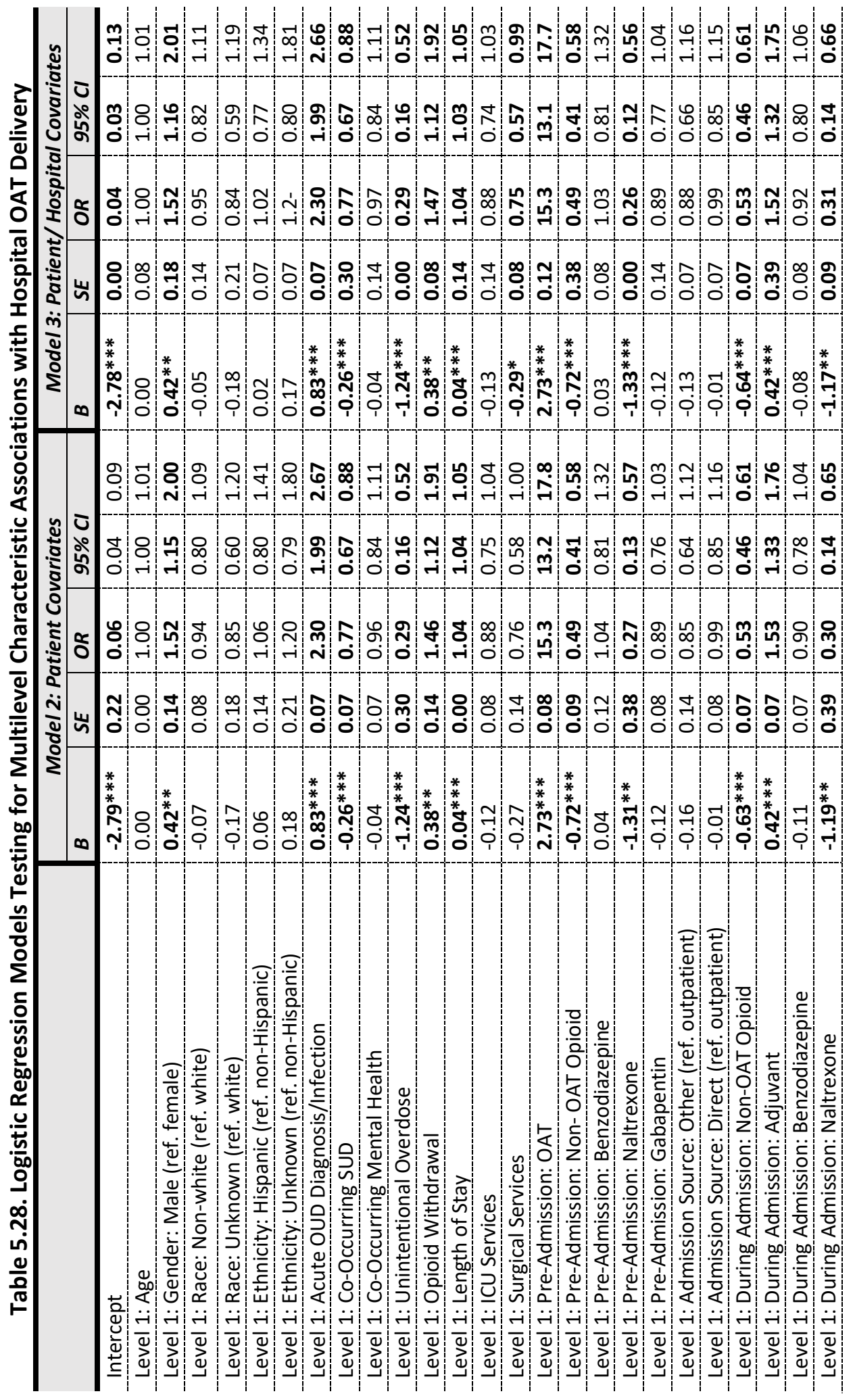




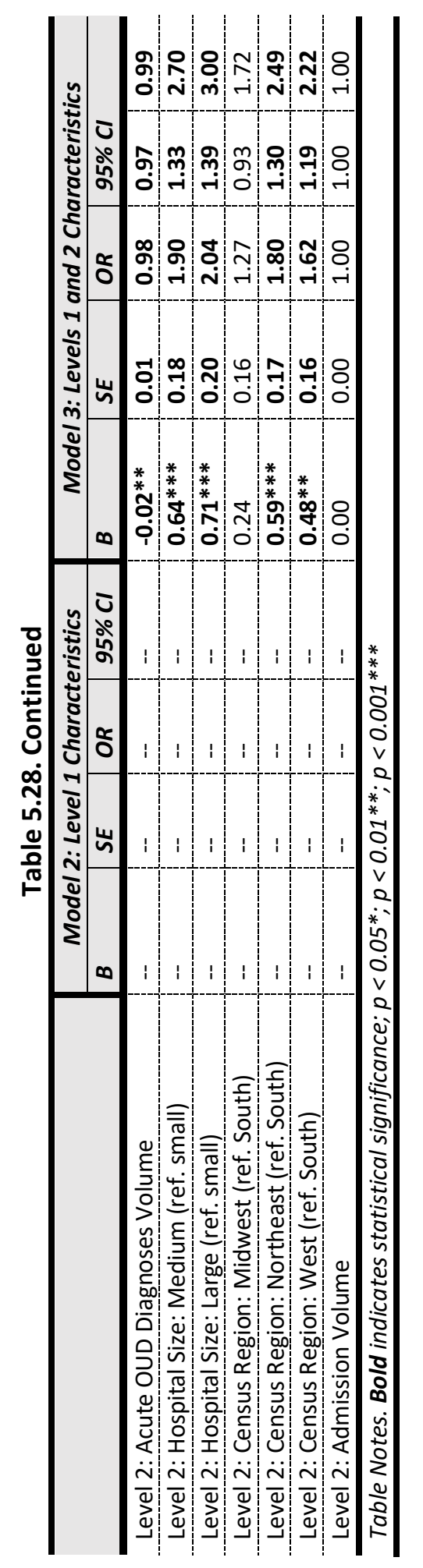


5.6.5. Summary. The multilevel model provided another perspective of how OAT delivery varied across the VHA system with two primary findings. First, unexplained variation was attributable to both the differences between hospitals (14\%) and differences within hospitals (86\%). In the final model, the between-hospital difference dropped to $6 \%$, but not $0 \%$, indicating that the patient and hospital characteristics included in the model did not fully explain between-hospital variation. Second, as hypothesized, several patient and hospital covariates were associated with differing odds of OAT receipt during admission. These findings reveal mutable and nonmutable characteristics for future exploration for patients, hospitals, and health systems. Patient receipt of OAT prior to hospitalization enhanced the odds of OAT during hospitalization indicating the importance of establishing OAT care in the outpatient setting prior to hospitalization and further, establishing care transition processes that enhanced care coordination for patients with OUD across the care delivery continuum. Specific immutable characteristics about the hospital, such as the geographic location and the size, likely reflected other unmeasurable attributes or resources within each respective hospital that influence the delivery of OAT.

A perplexing finding was that patients with acute opioid overdose diagnoses had lower odds of receiving OAT. One reason commonly used to explain the undertreatment of OUD in certain care delivery contexts is under diagnosis. In this study, however, patients with a primary or secondary overdose diagnosis had decreased odds of receiving this life-saving medication. The providers knew that these patients had an 
acute OUD issue; thus, the reasons for not providing OAT must be explained by something else (e.g., resources, training, culture, stigma).

\section{Section 5.7. Conclusion}

The primary hypotheses of this study, explored through qualitative methods and further addressed through quantitative approaches, were that care variation would exist for OAT delivery during hospital admission and that multilevel attributes (patient and hospital) would contribute to OAT delivery and OAT delivery variation between and within hospitals. Overall, the findings from Aim 2 and Aim 3 supported and extended the qualitative findings from Aim 1: 1) variability in OAT delivery existed in an integrated health system; 2) a severe care deficit existed for this patient population; and 3) mutable and nonmutable characteristics of patients and hospitals contribute to the delivery of OAT in the hospital setting. The final chapter, Chapter 6 , presents an integrated synthesis of the qualitative and quantitative findings. 


\section{Chapter 6. Discussion, Health System and Policy Implications, and Conclusions}

Parts of this chapter were published in a peer-reviewed journal: Priest, K.C., \& McCarty, D. (2018). Role of the hospital in the 21st Century opioid overdose epidemic: The addiction medicine consult service. Journal of Addiction Medicine, Advance online publication. doi: 10.1097/ADM.0000000000000496.

The final chapter of this dissertation integrates the results from the analysis of

the data supporting the three study aims with the overarching purpose of informing future research, practice, and policy. The study findings are timely and relevant as the U.S. struggles with the $21^{\text {st }}$ century opioid overdose epidemic. Prior to this dissertation, analyses describing care delivery for patients with an opioid use disorder (OUD) in the hospital were limited and there were no multi-site or system-wide assessments of the delivery patterns and practices for hospitalized patients with OUD. This study provides the first description of system-wide opioid agonist therapy (OAT) delivery and an exploration of the contextual attributes, inside and outside the hospital, associated with the delivery of services for hospitalized patients with OUD. The research question for this study asked: How do supply-side attributes influence hospital OAT delivery, health outcomes, and health services utilization for persons hospitalized with OUD?

To answer questions about complex social phenomena, such as hospital OAT delivery, the analysis drew on a diverse and complementary array of scholarship from the domains of public health, health policy, sociology, organizational behavior, systems science, and economics. The mixed methods study, grounded in a pragmatic research philosophy, addressed multiple OUD-related care delivery issues. The study provides a system wide quantitative analysis of the Veterans Health Administration (VHA) hospital 
services ( $n=109$ hospitals; $n=12,407$ patients) and a multisite qualitative assessment of non-VHA hospitals ( $\mathrm{n}=16$ non-VHA hospitals; $\mathrm{n}=17$ addiction medicine physicians) on the topic of hospital-based services for patients with OUD.

This final chapter includes a synopsis of the seven primary study findings, a presentation of the modified study conceptual framework, a discussion of research limitations, final conclusions, and the implications for health systems and policy:

1. Section 6.1 integrates the qualitative and quantitative results and concludes that OAT is underused in the hospital (Finding 1).

2. Section 6.2 outlines the evidence from the three study aims suggesting that OAT delivery varies across hospitals and health systems (Finding 2).

3. Section 6.3 describes how OAT is ineffectively used (e.g., withdrawal management) in hospitals inside and outside the VHA (Finding 3).

4. Section 6.4 illustrates how and which non-OAT modalities (e.g., short-acting opioid substitutes) are inappropriately used during and after hospitalization (Finding 4).

5. Section 6.5 provides evidence of specific attributes inside and outside the hospital facilitating or impeding AMC service development and OAT delivery (Finding 5).

6. Section 6.6 details patient attributes that impact hospital OAT delivery (Finding 6). 
7. Section 6.7 asserts, with evidence from this study, that the hospital is an important part of the OUD care continuum (Finding 7).

8. Section 6.8 presents the modified and updated study conceptual framework, based upon the study findings.

9. Section 6.9 outlines the limitations associated with the qualitative, quantitative, and integrative approaches.

10. Section 6.10 proposes health system and policy interventions related to education and service delivery to promote OAT use for hospitalized patients with OUD, and the removal of policies creating barriers to OAT access.

11. Section 6.11 outlines future research and policy questions.

12. Section 6.12 states the dissertation's final conclusions.

\section{Section 6.1. Finding 1: OAT is Underused in the Hospital}

Hospital OAT delivery for patients with OUD is essentially undescribed in the peer-reviewed literature; in contrast, OAT delivery in the community setting is wellstudied, and it is established that OAT is underused in that context (Knudsen \& Roman, 2012). The studies describing the hospital care delivery environment use discharge prescribing and referral data to assert potential hospital OAT underuse (Ali \& Mutter, 2016; Frazier et al., 2017; Naeger, Ali, et al., 2016; Rosenthal et al., 2015). To date, a multi-site or system-wide study has not been conducted to assess hospital OAT delivery. The findings from this study, therefore, are a novel contribution, confirming and expanding prior assertions that OAT is likely underused in the hospital. 
6.1.1. Qualitative evidence. Semi-structured interviews with 17 addiction medicine physicians, in non-VHA hospitals, documented OAT underutilization even in the hospitals with addiction medicine-related resources (e.g., addiction medicine trained physicians, or AMC services, or addiction medicine fellowships). OAT initiation (i.e., starting a patient on OAT who was not already on it prior to hospitalization), as federal guidance suggests (SAMHSA, 2018), should be offered by medical staff as the first-line treatment option for patients not already on OAT. In this study, informants perceived this practice, outside the AMC services, to be either uncommon or not occurring in non-VHA hospitals. One informant stated that their non-VHA hospital typically did not provide any OUD-related care, including symptom management for withdrawal.

6.1.2. Quantitative evidence. Across the VHA study cohort $(n=12,407)$ the vast majority of patients did not receive OAT during admission (85\%) or in the 30 days post admission (89\%). In this study cohort, a patient with an OUD who was hospitalized in a VHA hospital had a $12 \%$ chance of OAT administration during hospitalization, without controlling for patient or hospital characteristics. Hospital care practices across the VHA system reflected a lower than desired performance even in the highest performing hospitals. On average, less than one third of patients with OUD received OAT when treated in the highest OAT performing hospitals.

6.1.3. Summary. Evidence from the qualitative and quantitative analyses suggests that OAT is underused in the hospital context. This finding is supported by the 
perspectives of expert addiction medicine physicians in non-VHA hospitals and from the VHA health care administrative data. Further, there is outside evidence to suggest that the VHA system has enhanced hospital care quality as compared with non-VHA hospitals. VHA hospitals out-performed non-VHA hospitals on 14 of 15 care quality metrics (Weeks \& West, 2018). This may suggest that non-VHA hospitals have worse performance in this care delivery domain.

\section{Section 6.2. Finding 2: OAT Delivery Varies Across Hospitals and Health Systems}

There is a long-standing interest in identifying and explaining variation in health services delivery across communities (Glover, 1938). Low variation in health services delivery should occur for highly effective and inexpensive treatments (Chandra \& Skinner, 2012; Skinner, 2011; Wennberg et al., 2002), such as OAT. Variation in OAT use in the community setting, however, is associated with a variety of patient and structural elements (Ducharme, Knudsen, \& Roman, 2006; Mark, Lubran, McCance-Katz, Chalk, \& Richardson, 2015). Within the VHA system, in previous research, decreased OAT access was associated with older age, urban residence, and race (Manhapra, Quinones, \& Rosenheck, 2016). Variation in hospital OAT delivery within, among, or across health systems has not been assessed.

6.2.1. Qualitative evidence. Analysis of the hospital guidance documents received from the study's informants revealed variation in OAT delivery approaches across hospital contexts with modest similarities in practice. The withdrawal management hospital guidance documents differed in practice domains, specifically: 
which medication was first-line (i.e., methadone vs. buprenorphine vs. clonidine); severity of withdrawal symptoms (e.g., CoWS score $\geq 5$ or $\geq 8$ or $\geq 12$ or $\geq 13$ ); initial dose (e.g., methadone $5 \mathrm{mg}, 10 \mathrm{mg}$, or $15 \mathrm{mg}$ ); and the 24-hour maximum dose (e.g., 30 $\mathrm{mg}$ or $40 \mathrm{mg}$ of methadone). Similarly, variation was observed in the OAT initiation guidance documents (e.g., protocol initiation threshold, maximum total daily dose). In contrast, there was clear practice consensus for the acute management and non-acute pain management for patients on OAT prior to hospitalization. This was reflected in the guidance documents and the informant interviews. The consensus was that, in most circumstances, daily OAT should be continued in the hospital and that buprenorphine doses could be increased or split to help manage pain.

6.2.2. Quantitative evidence. The quantitative findings echo the qualitative findings. OAT delivery performance varied across the VHA health system. Hospital OAT delivery in this integrated health system ranged from $0 \%$ of admissions, in the lowest performing hospitals, to $43 \%$ of admissions, in the highest performing hospital. Hospital OAT delivery performance differed significantly across four hospital performance quartiles (Q4, Q3, Q2, and Q1). The median performance in the highest performing quartile (Q4) was $27 \%$ and the lowest performing quartile was $5 \%$, a five-fold difference in median care practices between the highest and lowest quartiles.

6.2.3. Summary. Evidence from the qualitative and quantitative analyses suggests that OAT delivery varies across hospitals and health systems in well-resourced non-VHA hospitals and within an integrated health system. Hospital OAT delivery 
variation within the VHA system is not surprising as it reflects previous OAT delivery research in the VHA's community-based treatment system (Finlay et al., 2018). In 2017, the overall rate for OUD pharmacotherapy delivery across the VHA was $41 \%$ ( $n=$ 22,179 ), with facility performance varying between $2 \%$ to $76 \%$ (Finlay et al., 2018); in this study, the median delivery across hospitals was $12 \%$, with hospital performance varying between $0 \%$ to $43 \%$ ( $n=109$ hospitals; $n=12,407$ patients). It is possible that hospitals and health systems outside this study sample have OAT delivery variation, in particular low resourced hospitals (e.g., no addiction medicine physicians) and hospitals not affiliated with an integrated health system.

\section{Section 6.3. Finding 3: Less Effective OAT Approaches Are Used}

To date, the peer-reviewed literature has not described or distinguished the different types of OAT delivery scenarios that could occur during hospitalization. This dissertation provides the first description and assessment of different types of hospital OAT scenarios, specifically: OAT continued; OAT initiation and linkage to care; OAT withdrawal management; and OAT sustained.

6.3.1. Qualitative evidence. The non-VHA key informants described OAT delivery in their respective hospitals as broadly focused on "detox" (i.e., withdrawing patients from opioids instead of initiating or continuing OAT) outside the care delivered by AMC services. The focus of OUD-related hospital technologies (e.g., hospital guidance documents) on withdrawal management in non-VHA hospitals supported this finding. Six of the non-VHA hospitals had guidance for OAT withdrawal management; in 
contrast, only three had OAT initiation guidance documents and four had OAT continuation guidance. One non-VHA hospital protocol was restricted to adjuvant symptom-only management (i.e., clonidine to minimize the severity of opioid withdrawal). This suggests that across non-VHA hospitals, even in the presence of addiction medicine experts, there may be a need for education on how to most effectively care for people with OUD during hospitalization to match contemporary research and federal guidance (SAMHSA, 2018). Medical withdrawal, unless it is patient preference, is contrary to known best practices for OUD treatment (Clark, Samnaliev, Baxter, \& Leung, 2011). Federal guidance suggests "offering maintenance therapy with medication, not short-term medically supervised withdrawal" (SAMHSA, 2018, pp. 3-44) and states that it is "essential for the patient to continue receiving OUD medication while hospitalized" (SAMHSA, 2018, pp. 3-101). Federal guidance further recommends that providers should not "force patients to withdraw from opioid agonist therapy in the hospital" (SAMHSA, 2018, pp. 3-104).

6.3.2. Quantitative evidence. The VHA quantitative findings corroborate the non-VHA qualitative findings that articulated the common use of withdrawal management. When OAT delivery occurred in the VHA system (in only $15 \%$ of hospital admissions), it was most commonly delivered as withdrawal management (39\%), which is not the recommended first-line approach to OUD management (SAMHSA, 2018). When separated into high and low OAT performance quartiles, the highest performing hospitals (Q4) were more likely than the other quartiles to use the other more effective 
OAT delivery approaches (e.g., OAT continued, OAT initiation and linkage, and OAT sustained). Nonetheless, the occurrence of these approaches was still infrequent in the Quartile 4 hospitals. Median performance in Quartile 4 was 10\% for OAT sustained, 3\% for OAT initiated and linkage to care, and $2 \%$, for OAT continued.

6.3.3. Summary. Evidence from the qualitative and quantitative analyses of this study suggests that less effective OAT approaches are used during hospitalization. This dissertation is the first study to attempt to distinguish four different hospital-based OAT delivery care scenarios: 1) OAT continued; 2) OAT initiated and linkage; 3) OAT sustained; and 4) OAT withdrawal management. It is possible that there are other OAT scenarios not captured in this study. These designations may serve as the beginning of a foundational conversation about hospital OAT delivery quality.

\section{Section 6.4. Finding 4: Non-OAT Pharmacotherapies are Used}

An additional care delivery concern related to the treatment of patients with OUD in the hospital is the possibility that other less effective non-OAT pharmacotherapies (e.g., short-acting non-OAT opioids) are used as a substitute for OAT. Research does not exist describing this explicit concern. Prior research, however, suggests that prescription non-OAT opioid use and other non-OAT medications (e.g., benzodiazepine) post-hospital discharge are common for patients with OUD (Larochelle et al., 2016; Naeger, Ali, et al., 2016). This study confirms these previously established findings and expands upon a literature gap to describe the frequent use of non-OAT opioid pharmacotherapy in the hospital for patients with OUD. 
6.4.1. Qualitative evidence. Since all of the key informants were addiction medicine experts, the use of non-OAT modalities as substitutes for OAT was not commonly described. Two informants, however, shared that their hospital withdrawal management protocols used non-OAT approved opioids, specifically: a rapid 72-hour taper using tramadol and an injectable buprenorphine taper protocol.

6.4.2. Quantitative evidence. Three findings from the VHA cohort suggest the potential use of non-OAT pharmacotherapies for the treatment of OUD. First, non-OAT opioid prescription or administration occurred more often than OAT delivery in all three time periods: 30 days prior to index hospitalization (30\% vs. $11 \%)$; during index hospitalization ( $55 \%$ vs. $15 \%$ ); and 30 days post-hospital admission (34\% vs. $11 \%$ ). Second, patients who were discontinued from their OAT during hospitalization were more likely to receive non-OAT opioids during admission (44\% vs. $26 \%$ ) and at discharge ( $27 \%$ vs. $12 \%)$ when compared with patients who were continued on their pre-OAT during hospitalization. Discontinuing OAT during hospitalization not only disrupted OUD treatment but appeared to promote the use of non-evidence-based care (i.e., the substitution of non-OAT opioids). Third, there were other potentially problematic prescribing trends post-admission, including: gabapentin/pregabalin (26\%), benzodiazepine (8\%), and concurrent benzodiazepine and opioid prescribing (4\%).

6.4.3. Summary. Evidence primarily from the quantitative analyses suggest that non-OAT pharmacotherapies, specifically, non-OAT opioids, may be administered and prescribed frequently within the VHA system. Post-admission opioid prescription 
frequency in the VHA cohort was higher (34\%) than previously reported findings within a non-VHA population, which had $22 \%$ of patients $(n=8,225)$ fill a non-OAT opioid prescription in the 30 days after hospital discharge (Naeger, Ali, et al., 2016). These findings suggest either clinician misidentification of patients with an OUD or provider training deficits. In most clinical scenarios, patients with OUD should not receive nonOAT opioid prescriptions upon discharge because non-OAT opioid continuation after a hospitalization is associated with higher risk for a subsequent overdose (Larochelle et al., 2016).

\section{Section 6.5. Finding 5: Contextual Hospital Attributes Inform OUD-Related Services}

Hospitals are socially constructed entities comprised of physical, technological, cultural, and social milieu (Scott, 2003). The internal social structures of organizations are comprised of normative, patterned, formal and informal interactions (Scott, 2003). Social structures within an organization impart explicit and implicit values, norms, and role expectations (Davis, 1949; Scott, 2003). These expectations are promulgated through reward systems for specific behavior and enacted through socialization, mentoring, group dynamics, decision-making, communication, influence, and leadership (Kinicki \& Kreitner, 2009; Ostroff et al., 2003). Organizations exist as coordinated systems embedded within broad complex networks (Meyer \& Rowan, 1977), dependent on external relationships (Scott, 2003).

In the health care context, a hospital exists as a functional unit within the nested context of a larger health services delivery production system and comprised of smaller 
units (patients, units, divisions, departments) (Ferlie \& Shortell, 2001; Perrow, 1986). A product of this phenomenon is the continuous evolution and interaction within each level of the nested context and the influence of external factors, such as other hospitals (DiMaggio \& Powell, 1983), the prior training and socialization of hospital employees (Scott, 2003), financial incentives or disincentives promoted through external contracts (Alchian \& Demsetz, 1972; Eisenhardt, 1989; Jensen \& Meckling, 1976; Zeckhauser \& Pratt, 1985), or the enforcement of contracts (North, 1990; Zeckhauser \& Pratt, 1985). This theoretical foundation provides the rationale and justification for exploring how attributes within and outside the hospital influence and shape the delivery of evidencebased OUD services. This study is the first to explore the attributes that facilitate or impede care for patients hospitalized with OUD.

6.5.1. Qualitative evidence. The analysis of the non-VHA key informant interviews found six internal facilitators, six internal barriers, seven external facilitators, and four external barriers to AMC service establishment and hospital OAT delivery. A primary finding was that the AMC service, a hospital characteristic, was both an output of a supportive hospital environment and a modifier for service delivery and the development of other addiction-related technologies. A supportive hospital environment was generated through positive social structures and technology and resources allocated to addiction-related service activities. Fifteen key informants described six supports provided at the 14 non-VHA hospitals with established or planned AMC services: 
- Historic precedent and investment in addiction service delivery, education, and research;

- Dedicated resources for addiction services, research, and education;

- Creation or existence of an addiction medicine clinical training program;

- Use of evidence-based addiction treatment practices;

- Leadership supportive of addiction treatment; and

- Internal stakeholders supportive of addiction treatment.

Key informants $(n=17)$ from all 16 non-VHA hospitals described AMC establishment and hospital OAT service delivery barriers. Informants most commonly cited barriers related to resources and staffing, social structures, and financing:

- Restrictive hospital policies and regulations that inhibited or prevented hospital OAT delivery;

- Bureaucratic processes slowing AMC service development, OUD-service delivery, and education;

- Limited staffing and resources for addiction-related treatment and education;

- Unsupportive internal stakeholders;

- Knowledge gaps within hospital leadership and clinical staff that promulgated discriminatory behavior towards patients with OUD; and

- Unstable internal financial environment jeopardizing AMC service sustainability. 
Each of the internal barriers and facilitators to AMC service and OAT delivery, except for historic precedent, are mutable properties of a hospital or health services delivery organization that could be changed through strategic interventions.

Key informants in hospitals with established or planned AMC services described seven external influencers that positively mediated AMC service establishment and subsequently enhanced OAT delivery:

- Elevation and prioritization of this issue by non-clinical stakeholders;

- Development of formal and informal partnerships with external clinical stakeholders;

- Access to a robust community-based treatment network;

- Media coverage of the opioid overdose epidemic;

- Policies and regulations enhancing OAT access;

- Supportive political leadership and political action; and

- Supportive financial incentives.

Four external barriers were also described by informants:

- A limited community-based treatment network;

- Policies and regulations impeding OAT access;

- Unsupportive political leadership and political action; and

- Financing and funding limitations.

These external facilitators and barriers were all mutable. Unlike interventions targeted at the hospital environment, however, hospital leadership and staff had limited 
control and influence to change these external elements. Changing, removing, increasing, promoting, or enhancing external elements is possible but likely a more challenging and long-term endeavor.

6.5.2. Quantitative evidence. The quantitative analyses did not support a nuanced assessment of organizational characteristics or the external hospital environments. Each hospital's OAT delivery performance, however, was compared to the other hospitals within the VHA system $(n=109)$ to discern hospital-level characteristics contributing to variation. The characteristics observed to enhance OAT delivery included hospital size and geographic location. An impediment to OAT delivery was an elevated rate of acute OUD admissions in the hospital. Statistically significant differences in hospital size and region were also observed among the hospital OAT performance quartiles. There were more small hospitals $(26 \% ; n=7)$ in the lowest performing quartile as compared with the hospitals in the highest performing quartile $(3 \% ; n=1)$. Most hospitals $(74 \% ; n=20)$ in the lowest performing quartile were in the South as compared with the highest performing quartile $(21 \% ; n=6)$.

Further, when patient and hospital characteristics were controlled for in the multilevel logistic regression model, specific hospital characteristics increased the odds of patient OAT receipt during admission: the medium (OR 1.90; 95\% $\mathrm{Cl}[1.33,2.70]$ ) and large hospital sizes (OR $2.04 ; 95 \% \mathrm{Cl}[1.39,3.00]$ ), as compared to the small hospitals, and hospitals located in the Northeast (OR 1.80; 95\% Cl [1.30, 2.49]) or the West (OR 1.62; $95 \% \mathrm{Cl}[1.19,2.22])$, as compared to the South. In contrast, elevated acute OUD 
diagnosis volume in a hospital decreased the odds of individual OAT receipt (OR 0.98;

$95 \% \mathrm{Cl}[0.97,0.99])$. Although the size or location of a VHA hospital is essentially immutable, these characteristics likely represent potentially modifiable organizational and environmental characteristics. The fully specified multilevel model accounted for only $6 \%$ (ICC value) of the contributions to performance variation in the VHA dataset. This suggests that there were other unmeasured organizational attributes contributing to OAT delivery variation within the VHA system.

6.5.3. Summary. Evidence from this study's qualitative and quantitative analyses suggests that internal and external supply-side elements likely influence both the establishment of AMC services and hospital OAT delivery. Research in the outpatient and community-based settings suggests a variety of reasons for OAT underuse (e.g., financial, regulatory, geographic, and health care professional attitudinal factors) (Sharma et al., 2017). This study is the first to describe these phenomena in the hospital setting.

\section{Section 6.6. Finding 6: Patient Attributes Facilitate or Deter Hospital OAT Delivery}

There is a limited evidence base suggesting that patients who are hospitalized with OUD or SUD would like to receive treatment for their drug use disorder. A qualitative analysis of 32 hospitalized adults with SUD, the majority of whom had moderate-to-high-risk use of opioids (65\%), concluded that current hospitals practice was not meeting their needs and that care could be greatly improved if life stressors, trauma, or basic needs were addressed (Velez et al., 2017). Patients articulated a need 
for supported, coordinated, and timely care upon hospital discharge (Velez et al., 2017). Studies of patient attributes that enhance or deter hospital OAT delivery have not been reported. This study builds on the findings that patient attributes contribute to hospital OAT delivery.

6.6.1. Qualitative evidence. In the interviews from non-VHA hospitals, informants perceived specific patient characteristics that were an impediment to OAT access. Informants described patients with OUD who inject drugs as particularly challenging to serve in the hospital because of their complex psychosocial characteristics (e.g., uninsured, homeless, criminal justice involvement) and ongoing medical issues (e.g., serious infections, polysubstance use). Patients with these challenges, specifically those with a need for long-term antibiotics, were also generally not accepted in transitional and lower acuity facilities, creating barriers for discharge. The common barriers to OAT, from this perspective, included insurance status, care disruption by the criminal justice system, the creation of a non-therapeutic atmosphere with law enforcement involvement, and the patient's own internalized stigma about OAT. In contrast, only one specific demographic characteristic emerged qualitatively to enhance OAT delivery-pregnancy. Pregnant patients had enhanced access to treatment because hospitals' efforts focused on providing protocol driven hospitalbased services with supported linkages to prevent premature births linked to maternal opioid withdrawal. 
6.6.2. Quantitative evidence. Associations between patient characteristics and hospital OAT delivery were observed in the VHA. The findings from the fully-specified multilevel logistic regression showed that patient demographics and the receipt of specific types of care before and during admission, were either positively or negatively associated with hospital OAT delivery. The positively associated patient characteristics included:

- An OUD diagnosis or infection during admission (OR 2.30; 95\% Cl [1.99, 2.66]);

- Male gender (OR 1.52; 95\% Cl [1.16, 2.01]); and

- An opioid withdrawal diagnosis (OR 1.47; 95\% $\mathrm{Cl}[1.12,1.92]$ ).

The other care delivery scenarios positively associated with hospital OAT delivery included:

- Pre-admission OAT receipt (OR 15.3; 95\% CI [13.2, 17.7]);

- Admission adjuvant receipt (OR 1.52; 95\% $\mathrm{Cl}[1.32,1.75])$; and

- An increased length of hospital stay (OR 1.04; 95\% $\mathrm{Cl}[1.03,1.05])$.

The negatively associated patient characteristics included:

- An unintentional overdose diagnosis (OR: 0.29; 95\% $\mathrm{Cl}[0.16,0.52]$ ); and

- Having a co-occurring SUD diagnosis during admission (OR: 0.77; 95\% Cl $[0.67,0.88])$.

The other care delivery scenarios negatively associated with hospital OAT delivery included: 
- Pre-admission non-OAT opioid receipt (OR 0.49; 95\% Cl [0.41, 0.58]);

- Admission non-OAT opioid receipt (OR 0.53; $95 \mathrm{Cl}[0.46,0.61]$ );

- Pre-admission naltrexone receipt (OR 0.26; $95 \% \mathrm{Cl}[0.12,0.56])$; and

- Admission naltrexone receipt (OR 0.31; 95\% $\mathrm{Cl}[0.14,0.66])$.

6.6.3. Summary. Evidence from this study's qualitative and quantitative analyses confirms and expands upon previous findings (Velez et al., 2017) that specific patient characteristics may facilitate or deter hospital-based OAT access. Demographic characteristics described as relevant to OAT access in the qualitative findings were either not assessed (e.g., criminal justice involvement, homelessness), not relevant to the veteran patient population (e.g., insurance status), or not common among patients in the VHA (e.g., pregnancy).

\section{Section 6.7. Finding 7: The Hospital has an Important Role in the OUD Care Continuum} There is a growing evidence base exploring the influence of hospital service delivery on patient outcomes upon discharge. Retrospective analyses of AMC services (Nordeck et al., 2018; Suzuki, 2016), prospective single-site studies (Trowbridge et al., 2017; Wakeman et al., 2017), and one randomized control trial (Liebschutz et al., 2014) suggest the provision of OUD-related services in the hospital context is associated with either discontinued or continued OAT upon discharge. Further, a recent study by Moreno et al. (2019) observed that 30-day and 90-day hospital readmissions were reduced for patients who received buprenorphine during their index hospitalization 
suggesting that care in the inpatient setting has implications post-discharge. The findings from this study build and expand on these previous findings.

6.7.1. Qualitative evidence. Data from the semi-structured interviews suggest that hospitals had a role in either disrupting, starting, or continuing care for patients with OUD. Hospital involvement was dependent on the existence of addiction-related resources, specifically the AMC service. Key informants, from non-VHA hospitals, shared that the AMC services were primarily responsible for OAT initiation. They observed that, when OAT initiation was managed by non-AMC providers, treatment gaps frequently occurred because of the providers' limited knowledge of how to link patients to care upon discharge. Care disruption was also attributed to restrictive internal policies related to OAT delivery and provider training gaps and confusion about OAT federal regulations. Providers and administrators commonly believed it was illegal to administer OAT in the hospital or that a provider had to have a special wavier.

6.7.2. Quantitative evidence. The VHA dataset illustrates how hospitals serve an important role in the broader OUD care continuum. There were 1,325 patients in the study cohort who received OAT prior to hospital admission, two-thirds (65\%; $n=867$ ) of these patients were continued on OAT during admission. This is a positive finding but also reflects room for improvement, as OAT discontinuation in the hospital setting should be a rare occurrence (e.g., patient preference). Of the 1,325 patients who received OAT prior to admission, $23 \%(n=308)$ did not receive OAT within 30 days of hospital discharge and their OAT was interrupted because of hospitalization. Of the 308 
patients whose OAT engagement was interrupted post-hospitalization, $53 \%$ had their OAT discontinued upon admission $(n=163)$, and $47 \%$ had their OAT continued during admission $(n=145)$. Further, patients who were continued on OAT were statistically less likely to leave the hospital against medical advice $(3 \% ; n=26)$ as compared to those who were discontinued on their OAT $(10 \% ; n=46)$. Further, for patients who received any type of OAT during admission were statistically more likely than those who did not receive any OAT during hospitalization to receive OAT in the post-admission period (48\% vs. $5 \% ; p<0.05)$. These observations suggest that hospitalization disrupts ongoing outpatient OAT delivery, inpatient medical management of the patient's primary issue, and OAT receipt upon hospital discharge.

6.7.3. Summary. Evidence from the study's qualitative and quantitative findings suggests that hospitals play an important organizational role in the OUD care continuum, either disrupting, starting, or continuing care. This perspective is reflected in the efforts of leadership at the Centers for Medicare \& Medicaid Services [CMS]). CMS requires the use of the ASAM levels of care (ASAM. 2018b) for $\S 1115$ waivers to address the opioid overdose epidemic (CMS, 2017), emphasizing care coordination among the different ASAM levels of care, including acute care hospitals (Level 4.0).

\section{Section 6.8. Modified Conceptual Framework.}

The original study conceptual framework, presented in Chapter 2 (Figure 2.1), guided research design, and analysis, through the integrated conceptualization of the contributions of the dynamic, evolving, organizational and socio-economic forces that 
influence the delivery of hospital-based OAT. Based on the results of the dissertation, the conceptual framework was updated and refined (see Figure 6.1). The most substantial modifications include: 1) visualizing the hospitalization of the patient as a dynamic interaction and exchange; 2) drawing attention to an organizational outcomethe establishment of an AMC service-that influences the OUD-related care delivered during hospitalization; 3) distinguishing different types of service delivery for patients with OUD; and 4) refining the broader categories of the contextual attributes. In addition to these changes, the modified conceptual framework became more granular through the incorporation of the specific findings from this study (i.e., how the hospital, the events of hospitalization, the patient demand-side attributes, and the external environment influence hospital OAT delivery, and AMC service establishment). The modified conceptual framework may help researchers and policymakers visualize the complex and interdependent contributions affecting the production and delivery of evidence-based services for hospitalized patients with OUD. The positive and negative contributions are included in the updated conceptual model. The (+) symbol identifies variables that enhance OAT delivery and support AMC services, and the (-) symbol reflects elements that inhibit OAT delivery and development of AMC services. The elements written in italics were quantitative findings and the non-italicized words were the qualitative findings. It is possible for an element to have both facilitative and impeding contributions and that contextual elements may fit into more than one category. 
External Supply-Side Attributes Social Structures

- Local politics (+/-)

- Media coverage (+)

Other organizations/stakeholder Clinical (+)

Community-based network (+/-)

Non-clinical (+)

\section{Technology \& Resources}

- Financing (+/-)

- Treatment policies (+/-)
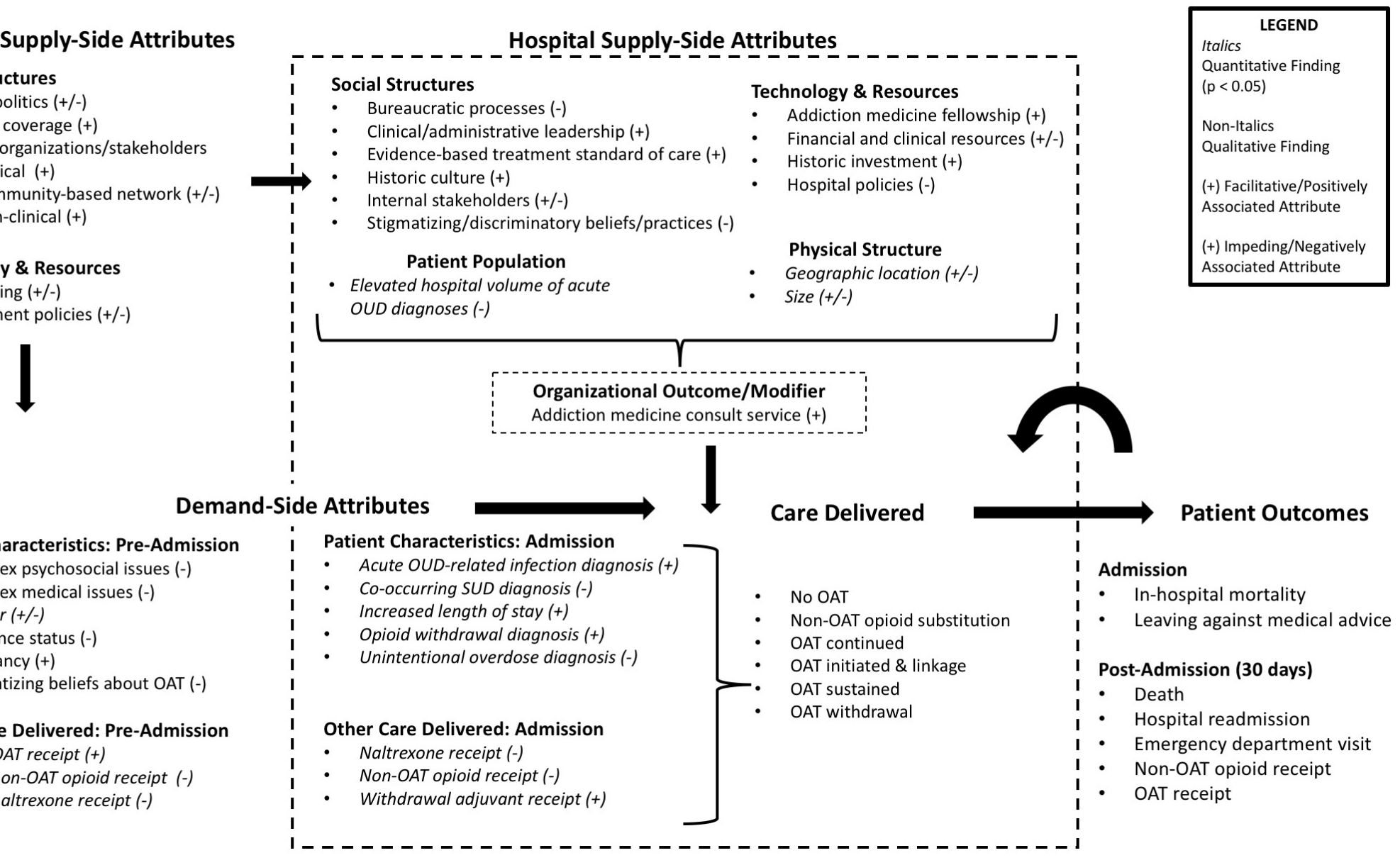

Figure 6.1. Updated Conceptual Framework

Patient Characteristics: Pre-Admission

- Complex psychosocial issues (-)

Complex medical issues (-)

- Gender (+/-)

Insurance status (-)

Pregnancy $(+)$

- Stigmatizing beliefs about OAT (-)

Other Care Delivered: Pre-Admission

- Prior OAT receipt (+)

- Prior non-OAT opioid receipt (-)

Prior naltrexone receipt (-)

\section{Figure 6.1. Updated Conceptual Framework}




\section{Section 6.9. Research Limitations}

The interpretation and conclusions generated from the findings of this dissertation are limited because of the study focus and the qualitative and quantitative methodological approaches.

6.9.1. Study scope limitations. This study was focused primarily on understanding and exploring supply-side contributions (i.e., the hospital) and not the demand-side contributions (i.e., the patient). This is a study finding limitation because health care access and delivery occurs through the dynamic interaction of the supplyside and demand-side (Levesque et al., 2013). The qualitative and quantitative datasets were limited to primarily exploring supply-side factors, aside from patient demographics. This was due to the choices made on how to reasonably scope this study, which included deciding on who to include in the qualitative study cohort. The study was limited to querying addiction medicine physician experts to ensure a narrower and more focused sampling frame to ensure timely completion of the dissertation. The inclusion of the patient perspective would have complicated the study design and methods (e.g., creation of patient survey, different sampling approach).

\subsubsection{Qualitative limitations. The main limitation of this study was}

transferability, which is inherent to findings from qualitative research. Findings from the interviews with addiction medicine experts in large well-resourced institutions may not be applicable to hospitals with more limited resources (e.g., the organizational and internal environmental attributes that facilitate AMC establishment and hospital OAT 
delivery). This study does not provide an understanding of the challenges faced in lower-resourced hospitals. Indeed, the proposed solution-the creation of AMC services - may not be possible in these contexts. Sixteen of the 17 informants were from hospitals not affiliated with an integrated delivery system, meaning that the organizational challenges particularly around care continuation differ from those working in a VHA type system. Further, this study cohort included perspectives from addiction medicine physicians at relatively well-resourced hospital with regards to addiction-related services.

Another limitation in this study was the variation in the amount of involvement that the key informants had with establishing or running their respective AMC services-some informants were observers, some were creators, and some worked on the service. This was a limitation because systems may be perceived differently based on the positionality of "the perceiver" (Lendaris, 1986). However, the quantitative analyses provided additional perspectives on the use of OAT among hospitalized patients that could more broadly translate to larger and smaller hospitals in rural and urban communities that serve a spectrum of patients. Finally, and importantly, this study did not include the patient perspective, which would have strengthened the study because patients would have identified relevant practice and policy issues unobserved in this study.

6.9.2. Quantitative limitations. There are also inherent limitations associated with retrospective secondary analyses of administrative health services data. First, as an 
observational retrospective cohort study, causal relationships cannot be established. Second, there were limitations to the generalizability of the results because of the study cohort (veterans) and the health services system design (the VHA). This was a study of VHA administrative data, and primarily applicable to the U.S. veteran population (older, white, and male). Further, the VHA is an integrated health payer and health system; thus, its linkages to care and organizational facilitators and barriers may differ from nonVHA hospitals, as was reflected in the sustained OAT data analyses. This is an important point because it means that third-party health insurance, the revenue generated through billing, and the formulary, which is the same across the VHA system, are not the external and internal environmental attributes contributing to deficiencies and variations in hospital OAT. Rather, other factors were contributing to variation in the VHA. In addition, patient inclusion criteria for quantitative aims included an OUD diagnosis in the year prior to (and including) index hospitalization. However, OUD may not have been a focus of treatment during hospitalization. Care practices may vary from what was observed in the current study when OUD-related complications are the primary reason for hospitalization. Finally, the study data are from the year 2017, a specific moment in time, and do not capture potential changes in practice over time. Nevertheless, the qualitative interviews with addiction medicine experts in non-VHA hospitals help balance the limitations in the quantitative analysis. The interviews offer insight into emerging standards of care and provide detail on how and why OAT is used for patients with OUD. 
The study cohort was also limited by the true denominator prevalence issue as described by Harris (2016). When using a diagnosis-based denominator-the number of persons hospitalized with OUD-to compare hospital delivery performance, it is possible that hospital delivery performance differences may be a result of a hospital's and clinical staff's ability to identify, diagnose, and document OUD. The findings from this study suggest that the contributions from these phenomena are likely minimal. Differences were not observed in the bivariate analyses of acute OUD diagnosis volume and OUD admission volume among hospital OAT performance quartiles. The true denominator prevalence issue is most problematic when the denominator affects performance measures. Selective identification of patients can enhance or suppress measures of hospital performance. The measures used in the quantitative analysis were not being used as quality measures and have no affect on hospital finances.

Another limitation is related to the construction of study variables, specifically, the health services utilization variables. The study was restricted to a 30-day period of observation post-index hospitalization. Previous research on opioid overdose deaths post-hospitalization used a time period longer than 30 days (Larochelle et al., 2018; Larochelle et al., 2016). A longer-term observation period (> 30 days) may provide additional information about risks of hospitalization and the OAT care delivery continuum. It is also possible that this study has underreported acute care delivery utilization in the 30-day period post-index hospitalization, as the 30-day ED and readmission flags only included readmissions and visits to VHA facilities. Finally, the 
study was likely underpowered to effectively assess the impact of infrequently occurring outcomes (e.g., 30-day death rate and in-hospital mortality).

6.9.3. Mixed methods integration limitations. There were several limitations to integrating the qualitative and quantitative findings for this mixed methods study. Key informants, and hospital guidance documents, were all from outside the VHA system. The quantitative data were from the VHA system. However, OAT care practice approaches outside the VHA informed the design of OAT practice variables and informed the selection of covariates included in the quantitative data. Unfortunately, data on the existence of AMC services or the presence of addiction-related hospitalbased services were not available for the VHA facilities, limiting the ability to connect VHA outcomes to VHA addiction-related hospital services. Nevertheless, mixing quantitative and qualitative data strengthened the conclusions of this research study. The final presentation and synthesis of study findings were enhanced because of the mixed methods approach used in this study. Importantly, the qualitative findings contextualized the quantitative findings, providing richer and policy and practice relevant applications. Further, the two sets of data were complimentary, confirmatory, and also provided insights that would not have emerged in isolation.

\section{Section 6.10. Health Systems and Policy Implications}

This section explores potential policy tools and health systems interventions that could enhance and promote health system and hospital services for patients with OUD. The evidence, provided by prior research paired with the findings from this study, 
suggests that OAT is not reaching the majority of hospitalized patients with an OUD and that systems-level attributes and policies inhibit hospital OAT delivery. This section proposes interventions for policymakers, health system and hospital leaders, clinicians, and researchers who are interested in improving services for patients hospitalized with OUD.

Policy tools are incentives or rules (Stone, 2011) that promote regulative or allocative action on policy targets (Dunn, 2012). Regulative policy action ensures compliance with certain standards or procedures, and allocative policy actions require the input of time, money, personnel, and equipment (Dunn, 2012). Policy tools may be distributive or redistributive (Lowi, 1964) and may target different actors responsible for action within a system. Health and social service policies are supposed to promote the non-punitive treatment for persons with SUDs (Babor et al., 2010). These policies affect treatment access through planning, financing, developing the professional workforce, and shaping the structural resources available to treat SUDs (e.g., number of facilities) (Klingemann et al., 1993; Klingemann \& Klingemann, 1999).

The findings from this study could inspire policy interventions inside and outside the hospital targeting a variety of actors to address OAT underuse, including: variation of OAT delivery across systems; the use of less effective treatment approaches; the frequent use of non-OAT modalities; the mutable internal and external environmental attributes impeding or facilitating hospital OAT delivery; the specific patient attributes that facilitate or impede OAT delivery; and the recognition that the hospital is an 
important component in the OUD care continuum. The suggested policy and health system interventions fall into three broad domains: 1) education; 2) service delivery; and 3) policy modifications.

6.10.1. Education interventions. An important observation from the non-VHA hospital informant interviews was the consensus across the study cohort that most hospital providers, in particular physicians, had knowledge gaps in their understanding of how to care for patients with OUD. Knowledge gaps included a lack of awareness of evidence-based practices for OUD treatment, misconceptions and discriminatory beliefs about people with SUDs, and a misunderstanding of federal OAT policies. There are at least two education-related strategies that may support the amelioration of knowledge gaps related to OUD and OAT service delivery: 1) targeted educational campaigns; and 2) educational requirements.

6.10.1a. Strategy 1: Targeted OAT educational campaigns. To reduce confusion related to federal OAT policies in the hospital setting, stakeholders could engage in educational initiatives to debunk common myths and to increase awareness about the legality and efficacy of providing OAT to hospitalized patients with OUD. SAMHSA could include specific information about these policies in its buprenorphine waiver training courses or create a special training and educational platform for hospital-based providers. Further, the DEA could improve messaging about the legality of treating patients with OAT during hospitalization and include this information in any documentation or re-licensing information related to a provider's general DEA license. 
Specific groups of providers could be targeted for intervention. Medical societies, in particular ASAM, could partner with the Society of Hospital Medicine and the American Hospital Association (AHA) to launch an educational campaign for hospital-based physicians. Further, orthopedic surgeons and emergency medicine physicians were particularly noted as having challenges in caring for patients with OUD. These specific groups could be a direct focus for policy initiatives and educational campaigns led by their respective medical societies: The American Board of Orthopaedic Surgeons and the American Academy of Emergency Medicine.

6.10.1b. Strategy 2: Educational requirements. There may also be opportunities for medical education regulatory and accrediting bodies (e.g., Association of American Medical Colleges, the Liaison Committee for Medical Education, Commission on Osteopathic College Accreditation, the American Osteopathic Association, and the Accreditation Council for Graduate Medical Education [ACGME]) to mandate evidencebased addictions training for both undergraduate and graduate medical education. The groups could incorporate training in addiction services into the learning competency requirements at each stage of medical training, and reinforce this learning with the inclusion of addiction medicine related questions in national medical licensing examinations.

Moreover, ACGME and physician specialty boards could leverage and expand upon programmatic requirements. Internal medicine residents, for example, are required to rotate at a hospital with a general medical consult service. This requirement 
means that general medical consult services must exist in hospitals that seek to employ internal medicine residents. Similarly, all internal medicine residents could be required to rotate on an AMC service. Another leverage point could be to require residents and medical students to obtain a buprenorphine waiver as a graduation requirement. This is being tested at the Brown University School of Medicine with support from state legislation (Arditi, 2017). This will likely become more common because the federal 2018 SUPPORT for Patients and Communities Act created a pathway for medical trainees to obtain a waiver to prescribe buprenorphine immediately upon entering practice (J. W. Frank, Wakeman, \& Gordon, 2018).

6.10.2. Service delivery interventions. There are at least four potential strategies to guide and enhance initiatives to improve OUD care in health systems and hospital settings: 1) guidance documents; 2) regulatory requirements; 3) statutory requirements; and 4) financial incentives.

6.10.2a. Strategy 1: Guidance documents. First, health systems and hospitals could implement standardized guidance documents to promote and enhance care delivery standards for hospital OAT delivery. Organizations can use guidance documents to address care variation to support standard practice in clinical scenarios with high levels of certainty, clinical agreement, and definitive science (Institute of Medicine Committee on Quality of Health Care in America, 2001). This intervention is proposed because significant variation was found in the quantitative and qualitative analyses. The clinical addiction medicine community could develop or endorse standard protocols and 
share these documents nationwide. AHA and ASAM could partner as initiative leaders to either create new materials or endorse the use of other materials already existence, such as the Project Support for Hospital Opioid Use Treatment (SHOUT) tools (Project SHOUT, 2018) or the recently published federal treatment guidance documents (Substance Abuse and Mental Health Services Administration, 2018). It is likely that the voluntary adoption of these guidance documents will be limited to the innovators and early adopters (Rogers, 2003); thus, the use of external coercive policies to adopt these guidelines may be necessary through the Joint Commission (see strategy 2 below).

6.10.2b. Strategy 2: Regulatory requirements. A second strategy to enhance OAT delivery may include the use of external coercive policies to require hospitals to deliver OAT by leveraging the power of hospital-related accrediting and professional regulatory bodies (e.g., the Joint Commission or American College of Surgeons [ACS]). The Joint Commission designates hospital accreditation requirements and ensures hospitals are meeting those standards. At present, there are no requirements related to the care of persons with OUD and SUDs in the hospital context. In 2008, the Joint Commission created optional performance metrics for alcohol screening and pharmacotherapy for persons with SUDs, including OUD (Joint Commission, 2015). It is within the authority of the Joint Commission to require reporting and performance measurement for OAT and mandate addiction-related technologies for hospital accreditation (e.g., presence of addiction medicine physicians or consult services). 
ACS is another regulatory body with the power to influence hospital practice, particularly at large tertiary care centers, or any hospital that strives to maintain or become trauma center certified. ACS specifically requires that all trauma patients admitted for more than 24 hours must be screened for alcohol use disorders (American College of Surgeons, 2018). The current policy could be expanded to include other SUDs or could be modified to require a consultation with a board-certified addiction medicine physician, which would require hospitals to have trained addiction medicine physicians on staff.

6.10.2c. Strategy 3: Statutory requirements. Third, local jurisdictions could enact coercive policies to improve hospital practices. An excellent example of this occurred recently in Massachusetts. In August 2018, the Massachusetts legislature passed House Bill 4866, Prevention and Access to Appropriate Care and Treatment of Addiction, comprehensive legislation focused on addressing the opioid overdose epidemic (WBUR News \& Wire Services, 2018). The legislation requires Massachusetts's emergency departments to offer and provide OAT for patients with an opioid overdose, and to link them to care upon discharge (WBUR News \& Wire Services, 2018). Similar legislation in other states could be proposed and expanded to require offering of OAT during hospital admission. Garnering the support necessary to pass this type of legislation would likely entail the creation of a broad coalition of patients, health care professionals, and legislators. An important consideration for this strategy is that there will be differences 
in how the statute is implemented and enforced in different jurisdictions, dependent on local governance structures among state health, mental health, and SUD agencies.

6.10.2d. Strategy 4: Financial incentives and penalties. Another strategy may be to create financial motivators for hospital performance related to OUD service delivery. Public payers, such as Medicaid, could incentivize appropriate hospital care through the Delivery System Reform Incentive Payment (DSRIP) program through the §1115 waiver mechanism (MACPAC, 2018). Through this program mechanism, policymakers could test reimbursement strategies, the design of interprofessional teams, penalties for delivering non-evidence-based services (e.g., funding detox beds), and financial incentives for evidence-based services. To make this a reality, CMS would need to explicitly outline these approaches in program notices for future demonstration opportunities.

Cities are also investing local dollars to create hospital AMC services. New York City Health \& Hospitals launched the Consult for Addiction Treatment and Care in Hospitals Program (CATCH). Funded in part by more than $\$ 7.5$ million from Mayor de Blasio's Healing NYC Initiative, the CATCH program is implementing AMC services at six New York public hospitals over the next one and a half years (NYC Health \& Hospitals, 2018). Replicating this citywide initiative in other jurisdictions is likely possible with the right coalition and project champions; however, similar to the variation in state law implementation and enforcement, a one-size-fits-all strategy might not be possible because of local differences in city and county health agency relationships, operations, and governance structures. 
Finally, the delivery of hospital-based OAT could be included in CMS pay-forperformance hospital programming such as Medicare's Hospital Value-Based Purchasing Program. This program uses value-based purchasing to redistribute funds to participating hospitals based on performance (CMS, 2018b) The metrics for this program could be updated to include care processes related to OAT delivery. Another potential CMS program that could incentivize OAT delivery during hospitalization is the Hospital Readmission Reduction Program (CMS, 2018a), which reduces hospital payments based on excessive readmissions. Conditions that are currently tracked do not include OUD and or OUD-related infection, and these could be added to the program. Summaries of the education and service delivery policy interventions are provided in Table 6.1, on the following page. 
Table 6.1. Interventions to Enhance OUD Services During Hospitalization

\begin{tabular}{|c|c|c|c|}
\hline $\begin{array}{l}\text { Policy Tool } \\
\text { and Strategy }\end{array}$ & $\begin{array}{l}\text { Policy } \\
\text { Action }\end{array}$ & $\begin{array}{l}\text { Responsible } \\
\text { Actors }\end{array}$ & $\begin{array}{l}\text { Target } \\
\text { Actors }\end{array}$ \\
\hline \multicolumn{4}{|l|}{ Education } \\
\hline $\begin{array}{l}\text { Targeted OAT educational } \\
\text { campaigns }\end{array}$ & N/A & $\begin{array}{l}\text { SAMHSA; DEA; } \\
\text { medical societies }\end{array}$ & $\begin{array}{l}\text { Hospital } \\
\text { physicians }\end{array}$ \\
\hline $\begin{array}{l}\text { Mandate addiction medicine } \\
\text { training as a core clinical } \\
\text { competency }\end{array}$ & Regulative & $\begin{array}{l}\text { UME regulatory } \\
\text { bodies }^{1} \text {; GME } \\
\text { regulatory bodies }\end{array}$ & $\begin{array}{l}\text { UME and GME } \\
\text { programs }\end{array}$ \\
\hline $\begin{array}{l}\text { Mandate addiction medicine } \\
\text { exam questions into licensing } \\
\text { exams }\end{array}$ & Regulative & NBME & Medical trainees \\
\hline $\begin{array}{l}\text { Mandate rotation on an AMC } \\
\text { service }\end{array}$ & Regulative & $\begin{array}{l}\text { GME regulatory } \\
\text { bodies }^{2}\end{array}$ & $\begin{array}{l}\text { GME programs; } \\
\text { health systems } \\
\text { and hospitals }\end{array}$ \\
\hline $\begin{array}{l}\text { Mandate } \\
\text { buprenorphine/naloxone training } \\
\text { for medical trainees }\end{array}$ & Regulative & $\begin{array}{l}\text { UME regulatory } \\
\text { bodies }^{1} \text {; GME } \\
\text { regulatory bodies }^{2}\end{array}$ & Medical trainees \\
\hline \multicolumn{4}{|l|}{ Service Delivery } \\
\hline $\begin{array}{l}\text { Create national hospital OAT } \\
\text { guidance documents and } \\
\text { mandate adoption }\end{array}$ & Regulative & $\begin{array}{l}\text { ASAM, AHA, Project } \\
\text { SHOUT, SAMHSA, Joint } \\
\text { Commission }\end{array}$ & $\begin{array}{l}\text { P\&T committees, } \\
\text { hospital legal } \\
\text { departments }\end{array}$ \\
\hline Mandate AMC service existence & Regulative & Joint Commission; ACS & $\begin{array}{l}\text { Health systems } \\
\text { and hospitals }\end{array}$ \\
\hline $\begin{array}{l}\text { Mandate hospital OUD care } \\
\text { quality reporting }\end{array}$ & Regulative & Joint Commission; ACS & $\begin{array}{l}\text { Health systems } \\
\text { and hospitals }\end{array}$ \\
\hline Mandate hospital OAT provision & Regulative & $\begin{array}{l}\text { State or local public } \\
\text { health department }\end{array}$ & $\begin{array}{l}\text { Health systems } \\
\text { and hospitals }\end{array}$ \\
\hline $\begin{array}{l}\text { Include OAT in the Hospital Value- } \\
\text { Based Purchasing Program }\end{array}$ & Allocative & CMS & $\begin{array}{l}\text { Health systems } \\
\text { and hospitals }\end{array}$ \\
\hline $\begin{array}{l}\text { Include OUD in the Hospital } \\
\text { Readmission Reduction Program }\end{array}$ & Allocative & CMS & $\begin{array}{l}\text { Health systems } \\
\text { and hospitals }\end{array}$ \\
\hline $\begin{array}{l}\text { Incentivize hospital OUD } \\
\text { treatment innovation with DSRIP } \\
\text { and } \$ 1115 \text { waiver mechanism }\end{array}$ & Allocative & CMS & $\begin{array}{l}\text { State Medicaid } \\
\text { program }\end{array}$ \\
\hline $\begin{array}{l}\text { Allocate local funds to pay for } \\
\text { AMC service }\end{array}$ & Allocative & Local jurisdictions & $\begin{array}{l}\text { Health systems } \\
\text { and hospitals }\end{array}$ \\
\hline \multicolumn{4}{|c|}{$\begin{array}{l}\text { Table Notes. SAMHSA = Substance Abuse and Mental Health Service Administration; DEA = Drug } \\
\text { Enforcement Administration; UME = undergraduate medical education; GME = graduate medical } \\
\text { education; NBME = National Board of Medical Examiners; AMC = addiction medicine consult service; } \\
\text { ASAM = American Society of Addiction Medicine; AHA = American Hospital Association; ACS = } \\
\text { American College of Surgeons; CMS = Centers for Medicare and Medicaid Services; DSRIP = Delivery } \\
\text { System Reform Incentive Payment; }{ }^{1} \text { American Association of Medical Colleges, the Liaison Committee } \\
\text { on Medical Education, the American Osteopathic Association; }{ }^{2} \text { Accreditation Council for Graduate } \\
\text { Medical Education and other medical specialty organizations. }\end{array}$} \\
\hline
\end{tabular}


6.10.3. Policy reform. Treatment policies, inside and outside non-VHA hospitals, were identified by key informants as creating OAT delivery barriers. Within the hospital, informants observed internal policies creating barriers through hospital formulary limitations, restrictive OAT prescribing and administering policies, and the absence of hospital-wide guidance documents for OUD-related services. The absence of guidance documents was perceived to complicate OUD care management issues because hospital standards of care for this patient population were unknown.

Policies creating barriers to hospital OAT access were created by third-party payers (e.g., coverage policies), OTPs (e.g., state assessment requirements), and federal drug treatment regulators (e.g., buprenorphine patient panel limits). Frequently, these policies negatively constrained outpatient OAT access, which in turn influenced the potential care delivery options in the hospital setting. Informants believed that streamlined access to outpatient services was necessary for establishing an AMC service (see Table 6.2).

Table 6.2. Policies for Reform Suggested by Informants

\begin{tabular}{|c|c|}
\hline Hospital & Outside the hospital \\
\hline \multirow[t]{3}{*}{$\begin{array}{l}\text { - Formulary restrictions on OAT } \\
\text { - Only allowing providers with DATA } \\
\text { waivers to prescribe } \\
\text { buprenorphine/naloxone }\end{array}$} & $\begin{array}{l}\text { Third-Party Payer Polices } \\
\text { - No methadone coverage } \\
\text { - Prior authorization for buprenorphine } \\
\text { - Utilization review for buprenorphine } \\
\text { - Formulary restrictions for buprenorphine }\end{array}$ \\
\hline & $\begin{array}{l}\text { OTP Policies } \\
\text { - State assessment requirements prior to placement } \\
\text { - Disallow concurrent polysubstance use }\end{array}$ \\
\hline & $\begin{array}{l}\text { Federal Drug Treatment Policies } \\
\text { - Buprenorphine panel limits }\end{array}$ \\
\hline
\end{tabular}


In addition to the suggestions for policy revision from informants, hospital policies such as behavioral management, visitor and room searches, and smoking policies could become more trauma-informed. Further, the removal of the 72 -hour rule, which limits the use of OAT for three days for patients admitted with opioid withdrawal, may eliminate much of the confusion held by hospital providers on the legality of providing OAT in the hospital. More broadly, policymakers could consider removing the special regulatory oversight system currently governing OAT, specifically the OTP regulations and buprenorphine/naloxone wavier system (Fiscella, Wakeman, \& Beletsky, 2018; J. W. Frank et al., 2018). Canada, for example, has discontinued its federal waiver and provider enrollment requirements for methadone to decrease administrative barriers to care (Priest et al., 2019). Despite decreasing federal government involvement, all the provinces still require or strongly suggest that providers who prescribe OAT participate in additional training (CRISM-ICRAS, 2018), which may still create barriers to OAT prescribing. Another international model with considerable success in increasing OAT access is the French system, which since 1996 has allowed providers to prescribe buprenorphine without any additional training or registration requirements (Auriacombe, Fatséas, Dubernet, Daulouede, \& Tignol, 2004). The U.S. could implement one of these approaches or a hybrid approach, either shifting responsibility to states or using the already existing federal regulatory system that oversees the distribution and prescription of scheduled pharmaceutical products (i.e., the FDA and the DEA). 


\section{Section 6.11. Future Research Questions and Areas for Exploration}

Several future research questions and areas for exploration were generated from study findings. Potential lines of inquiry include: extending and expanding upon this study; confirming the study's quantitative findings in non-VHA health systems and hospitals; confirming the study's qualitative findings in hospitals and health systems without an addiction medicine fellowship or in rural hospitals; operationalizing hospital OAT care quality; exploring barriers to provider knowledge; exploring demand-side issues from the patient perspective; and continuing research on AMC service design and operations.

6.11.1. Continue research with VHA hospitals. Future research could explore why hospital OAT variation exists across the VHA system. These explorations could include the addition of other information not in the original dataset, such as the existence of VHA affiliated community-based addiction-related services. Questions from this line of inquiry could include:

- What are the organizational or environmental (i.e., policy, regulatory, other organizations) factors that contribute to high performing VHA hospitals?

- What are the organizational or environmental (i.e., policy, regulatory, other organizations) factors that contribute to low performing VHA hospitals? Additional areas for exploration could involve a chart review to better understand why some patients were sustained or discontinued on OAT during hospitalization. This review could also include an investigation into hospital performance trends on 
sustaining OAT through a hospitalization. Another approach would be to see if different findings exist when the study population is narrowed to only patients hospitalized with opioid overdose or other OUD-related complications. Finally, different outcome measures related to OAT administration could be assessed through multilevel modeling including: in-hospital mortality, 30-day readmission, 30-day emergency department visit, 30-day death, and leaving the hospital against medical advice.

\subsubsection{Confirm and expand study findings in non-VHA hospitals. The} quantitative findings from this study could be confirmed outside the non-VHA system, specifically: Do the study's findings of OAT underuse, OAT variation, and the use of nonOAT pharmacotherapies persist in non-VHA hospitals and health systems, both in the U.S. or internationally? To answer this question, policymakers and researchers need to explore data access issues. One of the primary challenges to studying hospital OAT delivery is the widespread use of diagnosis-related groupings (DRG) in hospital billing. The DRG billing system allows hospitals to bill payers through a bundled payment algorithm to account for illness acuity (Quinn, 2008). Although DRG billing minimizes administrative billing processes, it unfortunately masks the ability for researchers and payers to determine if OAT was delivered during hospitalization, a conundrum for studying most pharmacotherapy administered during hospitalization. Another potential line of inquiry that bypasses the DRG billing complication could be to assess OAT delivery in the international hospital setting to determine if this care deficit exists in 
other care contexts or in other domestic integrated health systems (e.g., Kaiser Permanente).

\subsubsection{Confirm and expand study findings in hospitals without addiction}

medicine fellowships. The qualitative findings, in particular the barriers to AMC service establishment and the delivery of evidence-based care, could be confirmed in hospitals without addiction medicine fellowship affiliation and hospitals in rural settings. Questions for this avenue of research could include:

- Are there different contextual attributes that facilitate or impede AMC service establishment and OAT delivery in hospitals without an addiction medicine fellowship? Or in rural hospitals?

- Are the OAT and non-OAT practices different in hospitals without an addiction medicine fellowship? Or in rural hospitals?

6.11.3. Operationalize hospital OAT care quality. The findings from this study could lead to future lines of inquiry about how hospital OAT delivery quality could be defined and measured from a care quality perspective. Future questions could ask:

- Is the delivery of OAT during hospitalization enough or does linkage to care need to happen? What about patients who are already on OAT?

6.11.4. Explore barriers to provider knowledge. The findings from this study also inspire questions related to the knowledge base and training of health care professionals, in particular physicians. Future research could focus on exploring hospital provider understanding of contemporary best practices for OUD treatment and OAT 
regulations. A specific question that could be explored in the VHA setting is to assess why patients on OAT prior to hospitalization are being discontinued from OAT and being managed on short-acting opioid agonists. As was described in the qualitative findings, the continuation of OAT supplemented with short-acting opioids is the recommended approach to managing pain for patients with OUD (SAMHSA, 2018).

6.11.5. Explore demand-side perspectives. This study was designed to focus on supply-side barriers and facilitators; as such, future research could focus on the barriers to patient access to hospital OAT. This could be done by inviting the patient's perspective. Further, research could include and consider patient-informed harm reduction approaches and the impacts of hospitalization trauma. Recent research on hospitalized patients, not specific to patients with SUDs, suggests that the trauma of hospitalization itself is associated with increased access to acute care delivery services (ED visits and 30-day readmission) in the 30 days post discharge (Rawal et al., 2018). Based on the findings of this study and prior research (Velez et al., 2017), the trauma of hospitalization could disproportionately impact patients hosptialized with SUDs.

\subsubsection{Continue research on AMC service design and operations. Beyond this} study, little is known about the design and operations of AMC services. Future research could build on study findings by developing an AMC service design survey that could be administered by the AHA. This survey could further quantify the potential barriers to AMC service operations (e.g., questions related to hospital formulary), the prevalence of 
AMC services (e.g., does your hospital have an AMC service), and design elements (e.g., availability and coverage).

\section{Section 6.12. Study Conclusions and Future Challenges}

Addictions cannot be understood as merely the behavior patterns of individuals, as if they were the product of personal choices or personality characteristics alone, but also must be conceptualized as collective probabilities that are woven into the social fabric. (Reinarman \& Granfield, 2014, p. 16)

This study began the process of describing and exploring the dynamic, evolving, organizational and socio-economic phenomena influencing the delivery of hospitalbased services for patients with OUD. There are seven primary findings from this mixed methods study that confirm and expand upon prior research and also provide novel contributions. The quality of care for this patient population appears to be suboptimal (Findings 1, 2, 3, and 4). This study provides the first description of specific contextual attributes inside and outside the hospital that facilitate or impede AMC service development and hospital OAT delivery (Finding 5). This study builds upon a growing evidence base suggesting that patient attributes impede or facilitate hospital OAT delivery (Finding 6), and also provides a first look into the important role of the hospital in the broader OUD care continuum (Finding 7). The findings from this study suggest it is likely that many U.S. hospitals, particularly those without addiction medicine expertise, fail to provide adequate care for patients with OUD during hospitalization.

Although the study findings are primarily negative, there are a variety of ways in which hospitals, health systems, and policymakers may improve care for patients with OUD in the hospital setting. The proposed policy and system interventions have the 
potential to minimize harm and enhance support for people seeking treatment for their drug use. Resources should be allocated to these initiatives. There is, however, a significant challenge: the U.S. health services delivery system exists within the U.S. contemporary drug policy regime. The U.S. drug policy regime does not exist to minimize harm to persons who use drugs. It is grounded in a historical and social context that views persons with SUDs as morally deficient and incapable of making sound decisions. The construction of these views, embodied and normalized through over a century of policy and practice, run counter to the public health model of promoting human health and mitigating disease. A true public health approach to caring for persons with SUDs requires an overhaul of the U.S. drug policy regime. The current framework for addressing harmful drug use centers on the false notion that people who use drugs are immoral and must face criminal consequences for their behaviors. Until these antiquated views are stripped from federal and local policies, people with SUDs in the U.S. will continue to die and suffer unnecessarily. 


\section{References}

Academy of Managed Care Pharmacy. (2000). Principles of a sound drug formulary system. Retrieved from

http://amcp.org/WorkArea/DownloadAsset.aspx?id=9280

Aday, L. A., \& Andersen, R. M. (1974). A framework for the study of access to medical care. Health Services Research, 9(3), 208-220. Retrieved from https://www.ncbi.nlm.nih.gov/pmc/articles/PMC1071804/

Addiction Medicine Foundation. (2018). Addiction Medicine Fellowship Programs: 20182019. Retrieved from http://www.acaam.org/wpcontent/uploads/2018/11/Directory-of-Fellowships-2018-19-11-9-18.pdf

Agency for Healthcare Research and Quality. (2018). Beta Elixhauser Comorbidity Software for ICD-10-CM. Retrieved from https://www.hcupus.ahrq.gov/toolssoftware/comorbidityicd10/comorbidity icd10.jsp\#description

Ajzen, I., \& Fishbein, M. (1980). Understanding attitudes and predicting social behaviour. Englewood Cliffs, NJ: Prentice Hall, Inc.

Alchian, A. A., \& Demsetz, H. (1972). Production, information costs, and economic organization. The American Economic Review, 62(5), 777-795. doi:10.1109/EMR.1975.4306431

Alderks, C. E. (2017). Trends in the use of methadone, buprenorphine, and extendedrelease naltrexone at substance abuse treatment facilities: 2003-2015 (update). Retrieved from https://www.samhsa.gov/data/sites/default/files/report 3192/ShortReport3192.html

Ali, M. M., \& Mutter, R. (2016). Patients who are privately insured receive limited followup services after opiod-related hospitalizations. Retrieved from https://www.samhsa.gov/data/sites/default/files/report 2117/ShortReport2117.html

Allison, G. T., \& Zelikow, P. (1999). Essence of decision: Explaining the Cuban missile crisis (2nd ed.). New York, NY: Longman Publishing Group.

American College of Surgeons. (2018). Trauma verification: Q\&A web conference. Retrieved from https://www.facs.org/ /media/files/quality\%20programs/trauma/vrc\%20resour ces/vrc qa webinar slides feb2018.ashx

American Medical Association: Council on Mental Health. (1966). Review of the operation of the narcotic 'clinics' between 1919-1923. In J. O'Donnell \& J. Ball (Eds.), Narcotic addiction (pp. 180-187). New York, NY: Harper \& Row.

American Nurses Association. (2018). ANA commends U.S. Senate for passing opioid legislation package [Press release]. Retrieved from https://www.nursingworld.org/news/news-releases/2018/ana-commends-u.s.for-opioid-legislation/ 
American Psychiatric Association. (2013). Substance-related and addictive disorders Diagnostic and statistical manual of mental disorders: DSM 5 (5th ed.). Arlington, VA: American Psychiatric Association Publishing.

American Society of Addiction Medicine. (2011). Definition of addiction. Retrieved from https://www.asam.org/resources/definition-of-addiction

American Society of Addiction Medicine. (2016). The 21st Century Cures Act; Highlights for ASAM members. Retrieved from

https://www.asam.org/resources/publications/magazine/read/article/2016/12/ 07/the-21st-century-cures-act---highlights-for-asam-members

American Society of Addiction Medicine. (2018a). Nurse practitioners and physician assistants prescribing buprenorphine. Retrieved from https://www.asam.org/resources/practice-resources/nurse-practitioners-andphysician-assistants-prescribing-buprenorphine

American Society of Addiction Medicine. (2018b). What is the ASAM Criteria? Retrieved from https://www.asam.org/resources/the-asam-criteria/about

American Society of Health-System Pharmacists. (2008). ASHP statement on the pharmacy and therapeutics committee and the formulary system. Retrieved from https://www.ashp.org/-/media/assets/policy-

guidelines/docs/statements/pharmacy-and-therapeutics-committee-andformulary-system.ashx

Andersen, R., Davidson, P., \& Baumeister, S. E. (2013). Improving access to care. In G. F. Kominski (Ed.), Changing the US health care system: Key issues in health services policy and management (4th ed., pp. 33-69). San Francisco, CA: John Wiley \& Sons, Inc.

Arditi, L. (2017). Brown Medical School to pioneer addiction-treatment program. Retrieved from https://www.providencejournal.com/news/20170710/brownmedical-school-to-pioneer-addiction-treatment-program

Auriacombe, M., Fatséas, M., Dubernet, J., Daulouede, J.-P., \& Tignol, J. (2004). French field experience with buprenorphine. American Journal on Addictions, 13(sup1), S17-S28. doi:10.1080/10550490490440780

Aurin, M. (2000). Chasing the dragon: The cultural metamorphosis of opium in the United States, 1825-1935. Medical Anthropology Quarterly: International Journal for the Analysis of Health, 14(3), 414-441. doi:10.1525/maq.2000.14.3.414

Austin, P. C., \& Merlo, J. (2017). Intermediate and advanced topics in multilevel logistic regression analysis. Statistics in Medicine, 36(20), 3257-3277. doi:10.1002/sim.7336

Babor, T., Caulkins, J., Edwards, G., Fischer, B., Foxcroft, D., Humphreys, K., . . Strang, J. (2010). Drug policy and the public good. New York, NY: Oxford University Press.

Bacchi, C. (2012). Introducing the "What's the Problem Represented to be?" Approach. In A. Bletsas \& C. Beasley (Eds.), Engaging with Carol Bacchi: Strategic interventions and exchanges (pp. 21-24). Adelaide, Australia: The University of Adelaide Press. 
Baker-White, A. (2017). Emergency declarations and opioid overdose prevention. Association of State and Territorial Health Officials. Retrieved from http://www.astho.org/StatePublicHealth/Emergency-Declarations-and-OpioidOverdose-Prevention/6-8-17/

Ball, R. M. (1973). Social Security Amendments of 1972: Summary and legislative history. Retrieved from https://www.ssa.gov/policy/docs/ssb/v36n3/v36n3p3.pdf

Balu, S., Connor, P. O., \& Vogenberg, F. R. (2004). Contemporary issues affecting P\&T committees part 1: The evolution origin of formulary systems. P\&T: A PeerReviewed Journal for Managed Care \& Formulary Management, 29(11), 709-711. Retrieved from http://citeseerx.ist.psu.edu/viewdoc/download?doi=10.1.1.503.5216\&rep=rep1 $\underline{\text { \&type }=p d f}$

Bashshur, R. L., Shannon, G. W., \& Metzner, C. A. (1971). Some ecological differentials in the use of medical services. Health Services Research, 6(1), 61-75. Retrieved from https://www.ncbi.nlm.nih.gov/pmc/articles/PMC1067311/

Basole, R. C., \& Rouse, W. B. (2008). Complexity of service value networks: Conceptualization and empirical investigation. IBM Systems Journal, 47(1), 53-70. doi:10.1147/sj.471.0053

Beletsky, L. (2018). 21st Century Cures for the opioid crisis: Promise, impact, and missed opportunities. American Journal of Law \& Medicine, 44(2-3), 359-385. doi:10.1177/0098858818789417

Benyamin, R., Trescot, A. M., Datta, S., Buenaventura, R., Adllaka, R., Sehgal, N., . . Vallejo, R. (2008). Opioid complications and side effects. Pain Physician, 11(2 Suppl), S105-120. Retrieved from https://www.painphysicianjournal.com/linkout?issn=15333159\&vol=11\&page $=$ S105

Berger, P. L., \& Luckmann, T. (1966). The social construction of reality: A treatsie in the sociology of knowledge (1st ed.). Garden City, NY: Doubleday Publishing.

Berwick, D. M. (2003). Disseminating innovations in health care. JAMA, 289(15), 19691975. doi:10.1001/jama.289.15.1969

Besteman, K. J. (1992). Federal leadership in building the national drug treatment system. In D. Gerstein \& H. Harwood (Eds.), Treating drug problems: Volume 2: Commissioned papers on historical, institutional, and economic contexts of drug treatment (Vol. 2, pp. 63-88). Washington, DC: National Academies Press.

Birkland, T. A. (2015). An introduction to the policy process: Theories, concepts and models of public policy making. London, England: Routledge: Taylor \& Francis Group.

Bohnert, A. S., Ilgen, M. A., Galea, S., McCarthy, J. F., \& Blow, F. C. (2011). Accidental poisoning mortality among patients in the Department of Veterans Affairs Health System. Medical Care, 49(4), 393-396. doi:10.1097/MLR.0b013e318202aa27 
Bollinger, L. C., Burns, U. M., Chenault, K. I., Dolan, P. R., Izzo, R., \& Lane, J. B. (2012). Addiction medicine: Closing the gap between science and practice. Retrieved from

http://scholar.google.com/scholar?hl=en\&btnG=Search\&q=intitle:Addiction+Me dicine+:+Closing+the+Gap+between+Science+and+Practice\#7

Boyd, S., Carter, C. I., \& Macpherson, D. (2016). More harm than good: Drug policy in Canada. Black Point, Nova Scotia and Winnipeg, Manitoba: Fernwood Publishing.

Brown, P. (1995). Naming and framing: The social construction of diagnosis and illness. Journal of Health and Social Behavior, Spec No, 34-52. doi:10.2307/2626956

Campbell, N. D. (2010). The history of a public science: How the Addiction Research Center became the NIDA Intramural Research Program. Drug Alcohol Dependence, 107(1), 108-112. doi:10.1016/j.drugalcdep.2009.05.009

Campbell, N. D., Olsen, J. P., \& Walden, L. (2008). The narcotic farm: The rise and fall of America's first prison for drug addicts. Abrams, NY: Abrams Books.

Cantor, J. M. (1974). The alcohol and drug dependence program of the Veterans Administration. Journal of Drug Issues, 4(1), 44-51. doi:10.1177/002204267400400105

Caraceni, A., Hanks, G., Kaasa, S., Bennett, M. I., Brunelli, C., Cherny, N., . . . Zeppetella, G. (2012). Use of opioid analgesics in the treatment of cancer pain: Evidencebased recommendations from the EAPC. The Lancet Oncology, 13(2), e58-e68. doi:10.1016/S1470-2045(12)70040-2

Center for Behavioral Health Statistics and Quality. (2016). Key substance use and mental health indicators in the United States: Results from the 2015 National Survey on Drug Use And Health (HHS Publication No. SMA 16-4984, NSDUH Series H-51). Retrieved from http://www.samhsa.gov/data/

Centers for Disease Control and Prevention. (2018). Drug overdose deaths. Retrieved from https://www.cdc.gov/drugoverdose/data/statedeaths.html

Centers for Medicare \& Medicaid Services. (2017). SMD \# 17-003: RE: Strategies to Address the Opioid Epidemic. Retrieved from https://www.medicaid.gov/federal-policy-guidance/downloads/smd17003.pdf.

Centers for Medicare \& Medicaid Services. (2018a). Hospital Readmissions Reduction Program (HRRP). Retrieved from https://www.cms.gov/medicare/medicare-feefor-service-payment/acuteinpatientpps/readmissions-reduction-program.html

Centers for Medicare \& Medicaid Services. (2018b). The Hospital Value-Based Purchasing (VBP) Program. Retrieved from https://www.cms.gov/Medicare/Quality-Initiatives-Patient-AssessmentInstruments/Value-Based-Programs/HVBP/Hospital-Value-BasedPurchasing.html

Chaffee, E. E. (1985). Three models of strategy. Academy of Management Review, 10(1), 89-98. doi:10.5465/amr.1985.4277354

Chandler, A. (1962). Strategy and structure: Chapters in the history of the American enterprise. Cambridge, MA: Massachusetts Institute of Technology. 
Chandra, A., \& Skinner, J. (2012). Technology growth and expenditure growth in health care. Journal of Economic Literature, 50(3), 645-680. doi:10.1007/s13398-0140173-7.2

Christiansen, I. (2017). Commodification of healthcare and its consequences. World Review of Political Economy, 8(1), 82-103. doi:10.13169/worlrevipoliecon.8.1.0082

Church, A. (1900). Treatment of the opium habit by the bromide method. Quarterly Bulletin of the Northwestern University Medical School, 2(2), 422-430. Retrieved from https://www.ncbi.nlm.nih.gov/pmc/articles/PMC4301322/

Ciccarone, D. (2017). Fentanyl in the US heroin supply: A rapidly changing risk environment. The International Journal on Drug Policy, 46, 107-111. doi:10.1016/j.drugpo.2017.06.010

Clark, R. E., Samnaliev, M., Baxter, J. D., \& Leung, G. Y. (2011). The evidence doesn't justify steps by state Medicaid programs to restrict opioid addiction treatment with buprenorphine. Health Affairs, 30(8), 1425-1433. doi:10.1377/hlthaff.2010.0532

Clinard, M. B. (1957). The sociology of delinquency and crime. New York, NY: Reinhart \& Company.

Clinard, M. B., \& Meier, R. F. (2011). Sociology of deviant behavior (14th ed.). Boston, MA: Cengage Learning.

Committee on Pain Management and Regulatory Strategies to Address Prescription Opioid Abuse. (2017). Pain management and the opioid epidemic: Balancing societal and individual benefits and risks of prescription opioid use (R. J. Bonnie, M. A. Ford, \& J. K. Phillips Eds.): National Academies of Science, Engineering, Medicine.

Compton, W. M., \& Volkow, N. D. (2006). Abuse of prescription drugs and the risk of addiction. Drug and Alcohol Dependence, 83(Suppl 1), 4-7. doi:10.1016/j.drugalcdep.2005.10.020

CONSORT. (2010). The CONSORT flow diagram. Retrieved from http://www.consortstatement.org/consort-statement/flow-diagram

Cottler, L. B., Hu, H., Smallwood, B. A., Anthony, J. C., Wu, L. T., \& Eaton, W. W. (2016). Nonmedical opioid pain relievers and all-cause mortality: A 27-year follow-up from the epidemiologic catchment area study. American Journal of Public Health, 106(3), 509-516. doi:10.2105/AJPH.2015.302961

Courtwright, D. T. (2002). The roads to $\mathrm{H}$ : The emergence of the American heroin complex, 1898-1956. In D. F. Musto, P. Korsmeyer, \& T. W. Maulucci (Eds.), One hundred years of heroin (pp. 3-22). Westport, CT: Greenwood Publishing Group, Inc.

Courtwright, D. T. (2004). Drug wars: Policy hots and historical cools. Bulletin of the History of Medicine, 78(2), 440-450. doi:10.1353/bhm.2004.0066 
Courtwright, D. T. (2015). Preventing and treating narcotic addiction - A century of federal drug control. New England Journal of Medicine, 373(22), 2095-2097. doi:10.1056/NEJMp1508818

Covaleski, M. A., Dirsmith, M. W., \& Michelman, J. E. (1993). An institutional theory perspective on the DRG framework, case-mix accounting systems and healthcare organizations. Accounting Organizations and Society, 18(1), 65-80. doi:10.1016/0361-3682(93)90025-2

Creswell, J. W., \& Clark, V. L. P. (2011). Designing and conducting mixed methods research. Thousand Oaks, CA: SAGE Publications.

CRISM-ICRAS. (2018). CRISM national guideline for the clinical management of opioid use disorder. Retrieved from https://crism.ca/wpcontent/uploads/2018/03/CRISM NationalGuideline OUD-ENG.pdf

Crotty, M. (1998). The foundations of social research: Meaning and perspective in the research process. Thousand Oaks, CA: SAGE Publications.

Cummings, J. R., Wen, H., \& Ko, M. (2016). Decline in public substance use disorder treatment centers most serious in counties with high shares of black residents. Health Affairs, 35(6), 1036-1044. doi:10.1377/hlthaff.2015.1630

Cunningham, P., Rudowitz, R., Young, K., Garfield, R., \& Foutz, J. (2016). Understanding Medicaid hospital payments and the impact of recent policy changes. Retrieved from https://www.kff.org/medicaid/issue-brief/understanding-medicaidhospital-payments-and-the-impact-of-recent-policy-changes/

Davis, K. (1949). Human society. New York, NY: Macmillan Publishers.

De Jong, J. D. (2008). Explaining medical practice variation: Social organization and institutional mechanisms. Utrecht University, Utrecht University Repository.

DiMaggio, P. J., \& Powell, W. W. (1983). The iron cage revisited: Institutional isomorphism and collective rationality in organizational fields. American Sociological Review, 48(2), 147-160. doi:10.2307/2095101

DiMaggio, P. J., \& Powell, W. W. (1991). The new institutionalism in organizational analysis. Chicago, IL: University of Chicago Press

Dinno, A. (2017). Dunn.test: Dunn's Test of multiple comparisons using rank sums. Retrieved from https://CRAN.R-project.org/package=dunn.test

Donabedian, A. (1974). Aspects of medical care administration: Specifying requirements for health care. Health Services Research, 9(1), 86-87. doi:10.4236/abc.2014.44033

Dowell, D., Haegerich, T. M., \& Chou, R. (2016). CDC guideline for prescribing opioids for chronic pain - United States, 2016. JAMA, 315(15), 1624-1645. doi:10.1001/jama.2016.1464

Drug Enforcement Administration. (2005). Title 21 Code of Federal Regulations: Part 1306 - Prescriptions. Retrieved from https://www.deadiversion.usdoj.gov/21cfr/cfr/1306/1306 07.htm.

Drug Enforcement Administration. (n.d.). Drug scheduling. Retrieved from https://www.dea.gov/drug-scheduling 
Ducharme, L. J., Knudsen, H. K., \& Roman, P. M. (2006). Evidence-based treatment for opiate-dependent clients: Availability, variation, and organizational correlates. American Journal of Drug and Alcohol Abuse, 32(4), 569-576. doi:10.1080/00952990600920417

Dunn, W. N. (2012). Public policy analysis (5th ed.). New York, NY: Routledge: Taylor \& Francis Group.

Durán-García, E., Santos-Ramos, B., Puigventos-Latorre, F., \& Ortega, A. (2011). Literature review on the structure and operation of pharmacy and therapeutics committees. International journal of Clinical Pharmacy, 33(3), 475-483. doi:10.1007/s11096-011-9501-6

Dutton, D. (1986). Financial, organizational and professional factors affecting health care utilization. Social Science \& Medicine, 23(7), 721-735. doi:10.1016/02779536(86)90121-8

Eisenhardt, K. M. (1989). Agency theory: An assessment and review. Academy of Management Review, 14(1), 57-74. doi:10.5465/amr.1989.4279003

Executive Office of the President of the United States. (2014). National drug control strategy. Retrieved from https://obamawhitehouse.archives.gov/sites/default/files/ondcp/policy-andresearch/ndcs 2014.pdf.

Fanjiang, G., Grossman, J. H., Compton, W. D., \& Reid, P. P. (2005). Building a better delivery system: A new engineering/health care partnership. Washington, DC: National Academies Press.

Feldstein, P. J. (2005). Health care economics (6th ed.). Clifton Park, NY: Thomson Delmar Learning.

Feng, Y., He, X., Yang, Y., Chao, D., Lazarus, L. H., \& Xia, Y. (2012). Current research on opioid receptor function. Current Drug Targets, 13(2), 230-246. doi:10.2174/138945012799201612

Ferlie, E. B., \& Shortell, S. M. (2001). Improving the quality of health care in the United Kingdom and the United States: A framework for change. Milbank Quarterly, 79(2), 281-315. doi:10.1111/1468-0009.00206

Fingar, K. R., Skinner, H., Johann, J., Coenen, N., Freeman, W. J., \& Heslin, K. C. (2018). Statistical brief \#245: Geographic variation in substance-related inpatient stays across states and counties in the United States, 2013-2015. Retrieved from https://www.hcup-us.ahrq.gov/reports/statbriefs/sb245-Substance-InpatientStays-Across-US-Counties.pdf

Finlay, A. K., Binswanger, I. A., Timko, C., Smelson, D., Stimmel, M. A., Yu, M., . . Harris, A. H. S. (2018). Facility-level changes in receipt of pharmacotherapy for opioid use disorder: Implications for implementation science. Journal of Substance Abuse Treatment, 95, 43-47. doi:10.1016/j.jsat.2018.09.006 
Finlay, A. K., Harris, A. H., Rosenthal, J., Blue-Howells, J., Clark, S., McGuire, J., . . Oliva, E. (2016). Receipt of pharmacotherapy for opioid use disorder by justice-involved US Veterans Health Administration patients. Drug and Alcohol Dependence, 160, 222-226. doi:10.1016/j.drugalcdep.2016.01.013

Fiscella, K., Wakeman, S. E., \& Beletsky, L. (2018). Buprenorphine deregulation and mainstreaming treatment for opioid use disorder: $\mathrm{X}$ the $\mathrm{X}$ waiver. JAMA Psychiatry, Advance online publication. doi:10.1001/jamapsychiatry.2018.3685

Fisher, E. S., Wennberg, J. E., Stukel, T. A., \& Sharp, S. M. (1994). Hospital readmission rates for cohorts of Medicare beneficiaries in Boston and New Haven. New England Journal of Medicine, 331(15), 989-995. doi:10.1056/NEJM199410133311506

Florence, C. S., Zhou, C., Luo, F., \& Xu, L. (2016). The economic burden of prescription opioid overdose, abuse, and dependence in the United States, 2013. Medical Care, 54(10), 901-906. doi:10.1097/MLR.0000000000000625

Food and Drug Administration. (2002). Subutex and Suboxone approved to treat opiate dependence. Retrieved from https://www.fda.gov/Drugs/DrugSafety/PostmarketDrugSafetylnformationforPa tientsandProviders/ucm191521.htm

Foucault, M. (1966). Madness and civilization: A history of insanity in the age of reason. New York, NY: Pantheon Books.

Fox, J., \& Weisberg, S. (2011). Car. Thousand Oaks, CA: SAGE Publications. Retrieved from http://socserv.socsci.mcmaster.ca/jfox/Books/Companion

Frank, J. W., Wakeman, S. E., \& Gordon, A. J. (2018). No end to the crisis without an end to the waiver. Substance Abuse, 39(3), 263-265. doi:10.1080/08897077.2018.1543382

Frank, R. G., \& Pollack, H. A. (2017). Addressing the fentanyl threat to public health. The New England Journal of Medicine, 376(7), 605-607. doi:10.1056/NEJMp1615145

Frazier, W., Cochran, G., Lo-Ciganic, W., Gellad, W., Gordon, A. J., Chang, C.-C. H., \& Donohue, J. M. (2017). Medication-assisted treatment and opioid use before and after overdose in Pennsylvania Medicaid. JAMA, 318(8), 750-752. doi:10.1001/jama.2017.7818

Frenk, J., \& White, K. L. (1992). The concept and measurement of accessibility. In K. L. White (Ed.), Health services research: An anthology (pp. 842-855). Washington, DC: Pan American Health Organization.

Friedland, R., \& Alford, R. R. (1991). Bringing society back in: Symbols, practices and institutional contradictions. In P. J. DiMaggio \& W. W. Powell (Eds.), The new institutionalism in organizational anaylsis (pp. 232-266). Chicago, IL: University of Chicago Press.

Friedman, L. S. (2002). The microeconomics of public policy analysis. Princeton, NJ: Princeton University Press.

Galbraith, J. R. (1982). Designing complex organizations. Reading, MA: Addison-Wesley Company. 
Gellad, W. F., Good, C. B., \& Shulkin, D. J. (2017). Addressing the opioid epidemic in the United States: Lessons from the Department of Veterans Affairs. JAMA Internal Medicine, 177(5), 611-612. doi:10.1001/jamainternmed.2017.0147

Giddens, A. (1979). Central problems in social theory: Action, structure, and contradiction in social analysis. Berkeley, CA: University of California Press.

Gladden, R. M., Martinez, P., \& Seth, P. (2016). Fentanyl law enforcement submissions and increases in synthetic opioid-involved overdose deaths - 27 states, 20132014. Morbidity and Mortality Weekly Report, 65(33), 837-843. doi:10.15585/mmwr.mm6533a2

Glover, J. A. (1938). The incidence of tonsillectomy in school children. Proceedings of the Royal Society of Medicine: Section of Epidemiology and State Medicine, Aug 31(10), 1219-1236. doi:10.1093/ije/dym258

Goode, E. (2015a). Deviant behavior (10th ed.). New York, NY: Routledge: Taylor \& Francis Group.

Goode, E. (2015b). The sociology of deviance: An introduction. In E. Goode (Ed.), The handbook of deviance (5th ed., pp. 1-29). West Sussex, England: John Wiley \& Sons, Inc.

Goodrick, E., \& Salancik, G. R. (1996). Organizational discretion in responding to institutional practices: Hospitals and cesarean births. Administrative Science Quarterly, 41(1), 1-28. doi:10.2307/2393984

Gordon, A. J., Kavanagh, G., Krumm, M., Ramgopal, R., Paidisetty, S., Aghevli, M., . . . Liberto, J. (2011). Facilitators and barriers in implementing buprenorphine in the Veterans Health Administration. Psychology of Addictive Behaviors, 25(2), 215224. doi:10.1037/a0022776

Gordon, A. J., Trafton, J. A., Saxon, A. J., Gifford, A. L., Goodman, F., Calabrese, V. S., . . . Liberto, J. (2007). Implementation of buprenorphine in the Veterans Health Administration: Results of the first 3 years. Drug and Alcohol Dependence, 90(23), 292-296. doi:10.1016/j.drugalcdep.2007.03.010

Grogan, C. M., Andrews, C., Abraham, A., Humphreys, K., Pollack, H. A., Smith, B. T., \& Friedmann, P. D. (2016). Survey highlights differences in Medicaid coverage for substance use treatment and opioid use disorder medications. Health Affairs, 35(12), 2289-2296. doi:10.1377/hlthaff.2016.0623

Haddad, S., \& Mohindra, K. (2002). Access, opportunities and communities: Ingredients for health equity in the South. Paper presented at the Public Health and International Justice Workshop, New York, NY.

Hair, J., Tatham, R., Anderson, R. E., \& Black, W. (1998). Multivariate data analysis (5th ed.). Upper Saddle River, NJ: Prentice Hall, Inc.

Harris, A. H. S. (2016). The primitive state of quality measures in addiction treatment and their application. Addiction, 111(2), 195-196. doi:10.1111/add.13096

Hartung, D., Johnston, K., Geddes, J., Leichtling, G., Priest, K., \& PT, K. (2019). Buprenorphine coverage in the Medicare Part D program: 2007 to 2018. JAMA, Advance online publication. doi:10.1001/jama.2018.20391 
Hartwell, D., Colquitt, J., Loveman, E., Clegg, A., Brodin, H., Waugh, N., . . Mackenzie, L. (2005). Clinical effectiveness and cost-effectiveness of immediate angioplasty for acute myocardial infarction: Systematic review and economic evaluation In T. Walley (Ed.), NIHR Health Technology Assessment programme: Executive summaries (Vol. 9, pp. 1-99). South Hampton, NY: NIHR Journals Library.

Hassamal, S., Goldenberg, M., Ishak, W., Haglund, M., Miotto, K., \& Danovitch, I. (2017). Overcoming barriers to initiating medication-assisted treatment for heroin use disorder in a general medical hospital. Journal of Psychiatric Practice, 23(3), 221229. doi:10.1097/PRA.0000000000000231

Hastings, S. N., Smith, V. A., Weinberger, M., Schmader, K. E., Olsen, M. K., \& Oddone, E. Z. (2011). Emergency department visits in Veterans Affairs medical facilities. American Journal of Managed Care, 17(6 Spec No.), e215-223. Retrieved from https://www.ajmc.com/journals/issue/2011/2011-6-vol17n6/ajmc 11jun hastings e215to23

Hayes, R. D., Chang, C. K., Fernandes, A., Broadbent, M., Lee, W., Hotopf, M., \& Stewart, R. (2011). Associations between substance use disorder sub-groups, life expectancy and all-cause mortality in a large British specialist mental healthcare service. Drug and Alcohol Dependence, 118(1), 56-61. doi:10.1016/j.drugalcdep.2011.02.021

Herzig, S. J., Rothberg, M. B., Cheung, M., Ngo, L. H., \& Marcantonio, E. R. (2014). Opioid utilization and opioid-related adverse events in nonsurgical patients in U.S. hospitals. Journal of Hospital Medicine, 9(2), 73-81. doi:10.1002/jhm.2102

Hillman, A. J., Withers, M. C., \& Collins, B. J. (2009). Resource dependence theory: A review. Journal of Management, 35(6), 1404-1427. doi:10.1177/0149206309343469

Hoffmann, J. P. (1990). The historical shift in the perception of opiates: From medicine to social menace. Journal of Psychoactive Drugs, 22(1), 53-62. doi:10.1080/02791072.1990.10472197

Hoffmann, M. (2013). The right drug, but from whose perspective? A framework for analysing the structure and activities of drug and therapeutics committees. European Journal of Clinical Pharmacology, 69(SUPPL. 1), 79-87. doi:10.1007/s00228-013-1491-y

Hohenstein, K. (2001). Just what the doctor ordered: The Harrison Anti-Narcotic Act, the Supreme Court, and the federal regulation of medical practice, 1915-1919. Journal of Supreme Court History, 26(3), 231-256. doi:10.1111/1059-4329.00027

Homans, G. C. (1950). The human group. New York, NY: Harpers.

Hsieh, H-F., \& Shannon, S. E. (2005). Three approaches to qualitative content analysis. Qualitative Health Research, 15(9), 1277-1288. doi:10.1177/1049732305276687

Hsu, D. J., McCarthy, E. P., Stevens, J. P., \& Mukamal, K. J. (2017). Hospitalizations, costs and outcomes associated with heroin and prescription opioid overdoses in the United States 2001-12. Addiction, 112(9), 1588-1564. doi:10.1111/add.13795 
Hurley, R. A., Hayman, L. A., Taber, K. H., Martin, M., Hurley, R. A., \& Taber, K. H. (2007). Is opiate addiction associated with longstanding neurobiological changes? The Journal of Neuropsychiatry and Clinical Science, 19(3), 242-248.

doi:10.1176/jnp.2007.19.3.242

ICD10Data.com. (2018). The Web's Free 2019 ICD-10-CM/PCS Medical Coding Reference. Retrieved from https://www.icd10data.com/

Ingram, H., \& Schneider, A. L. (1990). Improving implementation through framing smarter statutes. Journal of Public Policy, 10(1), 67-88. doi:10.1017/S0143814X00004682

Ingram, H., \& Schneider, A. L. (1991). The choice of target populations. Administration \& Society, 23(3), 333-356. doi:10.1177/009539979102300304

Institute of Medicine Committee for the Substance Abuse Coverage Study. (1990). Treating drug problems. Volume 1: A study of effectiveness and financing of public and private drug treatment systems. Washington, DC: National Academies Press.

Institute of Medicine Committee on Federal Regulation of Methadone. (1995). Federal regulation of methadone treatment. Washington, DC: National Academies Press. Institute of Medicine Committee on Quality of Health Care in America. (2001). Crossing the quality chasm: A new health system for the 21st Century. Washington, DC: National Academies Press.

Jaffe, J. H. (1975). The maintenance option and the Special Action Office for Drug Abuse Prevention. Psychiatric Annals, 5(10), 12-39. doi:10.1080/01900698108524411

Jaffe, J. H. (1997). The history and current status of opiate agonist treatment. Paper presented at the NIH consensus development conference: Effective medical treatment of heroin addiction, Bethesda, MD.

Jaffe, J. H., \& O'Keeffe, C. (2003). From morphine clinics to buprenorphine: Regulating opioid agonist treatment of addiction in the United States. Drug and Alcohol Dependence, 70(2), S3-S11. doi:10.1016/S0376-8716(03)00055-3

Jasinski, D. R., Pevnick, J. S., \& Griffith, J. D. (1978). Human pharmacology and abuse potential of the analgesic buprenorphine: a potential agent for treating narcotic addiction. Archives of General Psychiatry, 35(4), 501-516. doi:10.1001/archpsyc.1978.01770280111012

Jensen, M. C., \& Meckling, W. H. (1976). Theory of the firm: Managerial behavior, agency costs and ownership structure. Journal of Financial Economics, 3(4), 305360. doi:10.1016/0304-405X(76)90026-X

Joint Commission. (2015). Substance use. Retrieved from https://www.jointcommission.org/substance use/

Jonnes, J. (1996). Hep-cats, narcs, and pipe dreams: A history of America's romance with illegal drugs. New York, NY: Scribner Book Company. 
Julius, D. (1976). NIDA's naltrexone research program. In D. Julius \& P. Renault (Eds.), Narcotic antagonists: Naltrexone progress report NIDA research monograph 9 (pp. 5-11). Rockville, MD: U.S. Department of Health, Education, and Welfare, Public Health Service, Alcohol, Drug Abuse; Mental Health Administration; National Institute on Drug Abuse.

Katz, D., \& Kahn, R. L. (1978). The social psychology of organizations (2nd ed.). New York, NY: John Wiley \& Sons, Inc.

Keeler, E. B., Rubenstein, L. V., Kahn, K. L., Draper, D., Harrison, E. R., McGinty, M. J., . . . Brook, R. H. (1992). Hospital characteristics and quality of care. JAMA, 268(13), 1709-1714. doi:10.1001/jama.1992.03490130097037

Kinicki, A., \& Kreitner, R. (2009). Organizational behavior: Key concepts, skills \& best practices (4th ed.). New York, NY: McGraw-Hill Irwin.

Kleber, H. D. (2002). Methadone: The drug, the treatment, the controversy. In D. F. Musto, P. Korsmeyer, \& T. W. Maulucci (Eds.), One hundred years of heroin (pp. 149-158). Westport, CT: Greenwood Publishing Group, Inc.

Klingemann, H. K., Holder, H. D., \& Gutzwiller, F. (1993). Research on and prevention of alcohol-related trauma: phases, patterns, and issues. Addiction, 88(7), 861-866. doi:10.1111/j.1360-0443.1993.tb02103.x

Klingemann, H. K., \& Klingemann, H. D. (1999). National treatment systems in global perspective. European Addiction Research, 5(3), 109-117. doi:10.1159/000018978

Knudsen, H. K., Abraham, A. J., \& Roman, P. M. (2011). Adoption and implementation of medications in addiction treatment programs. Journal of Addiction Medicine, 5(1), 21-27. doi:10.1097/ADM.0b013e3181d41ddb

Knudsen, H. K., \& Roman, P. M. (2012). Financial factors and the implementation of medications for treating opioid use disorders. Journal of Addiction Medicine, 6(4), 280-286. doi:10.1097/ADM.0b013e318262a97a

Kolodny, A., Courtwright, D. T., Hwang, C. S., Kreiner, P., Eadie, J. L., Clark, T. W., \& Alexander, G. C. (2015). The prescription opioid and heroin crisis: A public health approach to an epidemic of addiction. Annual Review of Public Health, 36, 559574. doi:10.1146/annurev-publhealth-031914-122957

Korthuis, P., McCarty, D., Weimer, M., Bougatsos, C., Blazina, I., Zakher, B., . . . Chou, R. (2017). Primary care-based models for the treatment of opioid use disorder: A scoping review. Annals of Internal Medicine, 166(4), 268-278. doi:10.7326/M162149

Kovac, V. B. (2014). The more the merrier: A multi-sourced model of addiction. In R. Granfield \& C. Reinarman (Eds.), Expanding addiction: Critical essays (pp. 296312). New York, NY: Routledge: Taylor \& Francis Group.

Kreek, M. J., \& Vocci, F. J. (2002). History and current status of opioid maintenance treatments: Blending conference session. Journal of Substance Abuse Treatment, 23(2), 93-105. doi:10.1016/S0740-5472(02)00259-3 
Kuhn, T. S. (1970). The structure of scientific revolutions (2nd ed.). Chicago, IL: University of Chicago Press.

Larochelle, M. R., Bernson, D., Land, T., Stopka, T. J., Wang, N., Xuan, Z., ... Walley, A. Y. (2018). Medication for opioid use disorder after nonfatal opioid overdose and association with mortality: A cohort study. Annals of Internal Medicine, 169(3), 137-145. doi:10.7326/M17-3107

Larochelle, M. R., Liebschutz, J. M., Zhang, F., Ross-Degnan, D., \& Wharam, J. F. (2016). Opioid prescribing after nonfatal overdose and association with repeated overdose: A cohort study. Annals of Internal Medicine, 164(1), 1-9. doi:10.7326/M15-0038.

League of Nations. (1925). Second opium convention. Retrieved from https://treaties.un.org/pages/ViewDetails.aspx?src=TREATY\&mtdsg no=VI-6a\&chapter=6\&clang= en.

League of Nations. (1931). Convention for limiting the manufacture and regulating the distribution of narcotic drugs. Retrieved from https://treaties.un.org/pages/ViewDetails.aspx?src=TREATY\&mtdsg no=VI-8a\&chapter=6\&clang= en.

Leavitt, H. J. (1962). Applied organizational change in industry: Structural, technological and humanistic approaches. Pittsburgh, PA: Carnegie Institute of Technology.

Lendaris, G. G. (1986). On systemness and the problem solver: Tutorial comments. IEEE Transactions on Systems, Man, and Cybernetics, 16(4), 603-610. doi:10.1109/TSMC.1986.289266

Leshner, A. I. (1997). Addiction is a brain disease, and it matters. Science, 278(5335), $45-$ 47. doi:10.1126/science. 278.5335 .45

Levesque, J-F., Harris, M. F., \& Russell, G. (2013). Patient-centred access to health care: Conceptualising access at the interface of health systems and populations. International Journal for Equity in Health, 12(18), 1-9. doi:10.1186/1475-9276$12-18$

Liebschutz, J. M., Crooks, D., Herman, D., Anderson, B., Tsui, J., Meshesha, L. Z., . . . Stein, M. (2014). Buprenorphine treatment for hospitalized, opioid-dependent patients: A randomized clinical trial. JAMA Internal Medicine, 174(8), 1369-1376. doi:10.1001/jamainternmed.2014.2556

Ling, W. (2016). A perspective on opioid pharmacotherapy: Where we are and how we got here. Journal of Neuroimmune Pharmacology, 11(3), 394-400. doi:10.1007/s11481-016-9663-z

Lipsitz, L. A. (2012). Understanding health care as a complex system: The foundation for unintended consequences. JAMA, 308(3), 243-244. doi:10.1001/jama.2012.7551

Lowi, T. J. (1964). American business, public policy, case-studies, and political theory. World Politics, 16(4), 677-715. doi:10.2307/2009452

Luke, D. A. (2004). Multilevel modeling, quantitative applications in the social sciences. Thousand Oaks, CA: SAGE Publications. 
MacCoun, R. J., \& Martin, K. D. (2008). Drug use and drug policy in a prohibition regime. SSRN Electronic Journal(April 2008). doi:10.2139/ssrn.1118460

MacCoun, R. J., Saiger, A. J., Kahan, J. P., \& Reuter, P. (1993). Drug policies and problems: The promises and pitfalls of cross-national comparison. Retrieved from https://www.rand.org/pubs/reprints/RP224.html

MACPAC. (2018). Delivery system reform incentive payment programs. Retrieved from https://www.macpac.gov/wp-content/uploads/2018/03/Delivery-SystemReform-Incentive-Payment-Programs.pdf.

Maddux, J. F. (1978). History of the hospital treatment programs, 1935-74. In W. R. Martin \& H. Isbell (Eds.), Drug addiction and the US Public Health Service (pp. 217-250). Rockville, MD: Department of Health, Education, and Welfare.

Manhapra, A., Quinones, L., \& Rosenheck, R. (2016). Characteristics of veterans receiving buprenorphine vs. methadone for opioid use disorder nationally in the Veterans Health Administration. Drug and Alcohol Dependence, 160, 82-89. doi:10.1016/j.drugalcdep.2015.12.035

Mark, T. L., Lubran, R., McCance-Katz, E. F., Chalk, M., \& Richardson, J. (2015). Medicaid coverage of medications to treat alcohol and opioid dependence. Journal of Substance Abuse Treatment, 55, 1-5. doi:10.1016/j.jsat.2015.04.009

Martin, W. R. (1977). General problems of drug abuse and drug dependence. In W. R. Martin (Ed.), Drug addiction I (Vol. 45, pp. 3-40). Berlin, Germany: Springer Publishing Company.

Martin, W. R. (1983). Pharmacology of opioids. Pharmacological Reviews, 35(4), 283323. Retrieved from http://pharmrev.aspetjournals.org/content/pharmrev/35/4/283.full.pdf

Mattick, R. P., Breen, C., Kimber, J., \& Davoli, M. (2003). Methadone maintenance therapy versus no opioid replacement therapy for opioid dependence. Cochrane Database of Systematic Reviews, 2(CD002209). doi:10.1002/14651858.CD002209

Mattick, R. P., Breen, C., Kimber, J., \& Davoli, M. (2009). Methadone maintenance therapy versus no opioid replacement therapy for opioid dependence. Cochrane Database of Systematic Reviews, 8(3), CD002209. doi:10.1002/14651858.CD002209.pub2

Mattick, R. P., Breen, C., Kimber, J., \& Davoli, M. (2014). Buprenorphine maintenance versus placebo or methadone maintenance for opioid dependence. Cochrane Database of Systematic Reviews, 2, CD002207. doi:10.1002/14651858.CD002207.pub4.

McAllister, W. B. (2004). The global political economy of scheduling: The internationalhistorical context of the Controlled Substances Act. Drug and Alcohol Dependence, 76(1), 3-8. doi:10.1016/j.drugalcdep.2004.02.012

McCarty, D., Priest, K. C., \& Korthuis, P. T. (2018). Treatment and prevention of opioid use disorder: Challenges and opportunities. Annual Review of Public Health, 39, 525-541. doi:10.1146/annurev-publhealth-040617-013526 
Merrall, E. L. C., Kariminia, A., Binswanger, I. A., Hobbs, M. S., Farrell, M., Marsden, J., . . . Bird, S. M. (2010). Meta-analysis of drug-related deaths soon after release from prison. Addiction, 105(9), 1545-1554. doi:10.1111/j.1360-0443.2010.02990.x

Mettler, S., \& SoRelle, M. (2014). Policy feedback theory. In P. A. Sabatier \& C. M. Weible (Eds.), Theories of the Policy Process (pp. 151-181). Boulder, CO: Westview Press.

Meyer, J. W., \& Rowan, B. (1977). Institutionalized organizations: Formal structure as myth and ceremony. American Journal of Sociology, 83(2), 340-363. doi:10.1086/226550

Microsoft. (2018). Microsoft Excel for Mac (Version 16.16.5).

Midi, H., Sarkar, S., \& Rana, S. (2010). Collinearity diagnostics of binary logistic regression model. Journal of Interdisciplinary Mathematics, 13(3), 253-267. doi:10.1080/09720502.2010.10700699

Mitchell, D., Mitchell, C., \& Ofshe, R. (1980). The light on Synanon: How a country weekly exposed a corporate cult--and won the Pulitzer Prize. New York, NY: Seaview Press.

Moreno, J. L., Wakeman, S. E., Duprey, M. S., Roberts, R. J., Jacobson, J. S., \& Devlin, J. W. (2019). Predictors for 30-Day and 90-Day hospital readmission among patients with opioid use disorder. Journal of Addiction Medicine, Advance online publication. doi:10.1097/ADM.0000000000000499

Morgan, D. L. (2014). Integrating qualitative and quantitative methods: A pragmatic approach. Thousand Oaks, CA: SAGE Publications.

Moynihan, D. P. (2002). One hundred years of heroics. In D. F. Musto, P. Korsmeyer, \& T. W. Maulucci (Eds.), One hundred years of heroin (pp. 23-38). Westport, CT: Greenwood Publishing Group, Inc.

Musto, D. F. (1973). The American disease: Narcotics in nineteenth century America. New Haven, CT: Yale University Press.

Musto, D. F. (1987). The history of legislative control over opium, cocaine, and their derivatives. In R. Hamowy (Ed.), Dealing with drugs: Consequences of government control (pp. 37-50). San Francisco, CA: Pacific Research Institute for Public Policy.

Musto, D. F. (1999). The American disease: Origins of narcotic control. New York, NY: Oxford University Press.

Musto, D. F. (2002). Introduction: The origins of heroin. In D. F. Musto, P. Korsmeyer, \& T. W. Maulucci (Eds.), One hundred years of heroin (pp. xiii-xvii): Greenwood Publishing Group, Inc.

Musumeci, M., \& Tolbert, J. (2018). Federal legislation to address the opioid crisis: Medicaid provisions in the SUPPORT Act. Henry J Kaiser Family Foundation. Retrieved from https://www.kff.org/medicaid/issue-brief/federal-legislation-toaddress-the-opioid-crisis-medicaid-provisions-in-the-support-act/ 
Naeger, S., Ali, M. M., Mutter, R., Mark, T., \& Hughey, L. (2016). Prescriptions filled following an opioid-related hospitalization. Psychiatric Services, 67(11), 12621264. doi:10.1176/appi.ps.201500538

Naeger, S., Mutter, R., Ali, M. M., Mark, T., \& Hughey, L. (2016). Post-discharge treatment engagement among patients with an opioid-use disorder. Journal of Substance Abuse Treatment, 69, 64-71. doi:10.1016/j.jsat.2016.07.004

Nagel, L. (2002). Emergency narcotic addiction treatment. Retrieved from https://www.deadiversion.usdoj.gov/pubs/advisories/emerg treat.htm.

National Center for Health Statistics. (2017). Health expenditures. Retrieved from https://www.cdc.gov/nchs/fastats/health-expenditures.htm

Nielsen, S., Larance, B., Degenhardt, L., Gowing, L., Kehler, C., \& Lintzeris, N. (2016). Opioid agonist treatment for pharmaceutical opioid dependent people. Cochrane Database of Systematic Reviews(5), CD011117. doi:10.1002/14651858.CD011117.pub2

Nixon, R. (1973). Executive Order 11727-Drug Law Enforcement [Press release]. Retrieved from http://www.presidency.ucsb.edu/ws/index.php?pid=59117

Nordeck, C. D., Welsh, C., Schwartz, R. P., Mitchell, S. G., Cohen, A., O'Grady, K. E., \& Gryczynski, J. (2018). Rehospitalization and substance use disorder (SUD) treatment entry among patients seen by a hospital SUD consultation-liaison service. Drug and Alcohol Dependence, 186, 23-28.

doi:10.1016/j.drugalcdep.2017

North, D. C. (1990). Institutions, institutional change and economic performance. Cambridge, MA: Cambridge University Press.

Noska, A., Mohan, A., Wakeman, S., Rich, J., \& Boutwell, A. (2015). Managing opioid use disorder during and after acute hospitalization: A case-based review clarifying methadone regulation for acute care settings. Journal of Addictive Behaviors, Therapy \& Rehabilitation, 4(2), pii: 000138. doi:10.4172/2324-9005.1000138

NYC Health \& Hospitals. (2018). NYC Health + Hospitals to engage 8,000 more patients with opioid use disorder in communities hardest hit by the opioid epidemic [Press release]. Retrieved from https://www.nychealthandhospitals.org/pressrelease/six-hospitals-engaging8000-more-patients-with-opioid-use-disorder/

O’Donnell, J., \& Vogenberg, F. R. (2012). Policies and procedures: Enhancing pharmacy practice and limiting risk. Pharmacy and Therapeutics, 37(6), 341-344. Retrieved from https://www.ncbi.nlm.nih.gov/pmc/articles/PMC3411206/

Oliva, E. M., Harris, A. H., Trafton, J. A., \& Gordon, A. J. (2012). Receipt of opioid agonist treatment in the Veterans Health Administration: Facility and patient factors. Drug and Alcohol Dependence, 122(3), 241-246. doi:10.1016/j.drugalcdep.2011.10.004

Oliva, E. M., Maisel, N. C., Gordon, A. J., \& Harris, A. (2011). Barriers to use of pharmacotherapy for addiction disorders and how to overcome them. Current Psychiatry Reports, 13(5), 374-381. doi:10.1007/s11920-011-0222-2 
Oliva, E. M., Trafton, J. A., Harris, A. H., \& Gordon, A. J. (2013). Trends in opioid agonist therapy in the Veterans Health Administration: Is supply keeping up with demand? The American Journal of Drug and Alcohol Abuse, 39(2), 103-107. doi:10.3109/00952990.2012.741167

Ostroff, C., Kinicki, A. J., \& Tamkins, M. M. (2003). Organizational culture and climate. Handbook of Psychology. doi:10.1002/0471264385.wei1222

Penchansky, R., \& Thomas, J. W. (1981). The concept of access: Definition and relationship to consumer satisfaction. Medical Care, 19(2), 127-140. doi:10.1097/00005650-198102000-00001

Perrow, C. (1986). Complex organizations: A critical essay (3rd Ed.). New York, NY: Random House.

Peters, D. H., Garg, A., Bloom, G., Walker, D. G., Brieger, W. R., \& Hafizur Rahman, M. (2008). Poverty and access to health care in developing countries. Annals of the New York Academy of Sciences, 1136(1), 161-171. doi:10.1196/annals.1425.011

Pfeffer, J., \& Salancik, G. R. (1978). The external control of organizations: A resource dependence perspective. New York, NY: Harper \& Row.

Pfohl, S. J. (1994). Images of deviance and social control: A sociological history (2nd ed.). New York, NY: McGraw-Hill

Pierson, P. (1993). When effect becomes cause: Policy feedback and political change. World Politics, 45(4), 595-628. doi:10.2307/2950710

Plsek, P. E., \& Greenhalgh, T. (2001). Complexity science: The challenge of complexity in health care. BMJ, 323(7313), 625-628. Retrieved from 10.1136/bmj.323.7313.625

Priest, K. C., Gorfinkel, L., Klimas, J., Jones, A., Fairbairn, N., \& McCarty, D. (2019). Comparing Canadian and United States opioid agonist therapy policies. International Journal of Drug Policy, Advance online publication. doi:10.1016/j.drugpo.2019.01.020

Priest, K. C., Leof, A., McCarty, D., \& King, V. (2017). Medicaid coverage for residential substance use disorder treatment: Addressing the institution for mental disease exclusion policy [Blog post]. Retrieved from http://www.healthaffairs.org/do/10.1377/hblog20170831.061745/full/

Priest, K. C., \& McCarty, D. (2018). Role of the hospital in the 21st Century opioid overdose epidemic: The addiction medicine consult service. Journal of Addiction Medicine, Advance online publication. doi:10.1097/ADM.0000000000000496

Project SHOUT. (2018). SHOUT: Support for Hospital Opioid Use Treatment. Retrieved from https://www.projectshout.org/

Quinn, K. (2008). New directions in Medicaid payment for hospital care. Health Affairs, 27(1), 269-280. doi:10.1377/hlthaff.27.1.269

Rawal, S., Kwan, J. L., Razak, F., Detsky, A., Guo, Y., Lapointe-Shaw, L., . . . Verma, A. (2018). Association of the trauma of hospitalization with 30-day readmission or emergency department visit. JAMA Internal Medicine, Advance online publication. doi:10.1001/jamainternmed.2018.5100 
Reddish, W. (1931). The treatment of morphine addiction by blister fluid injection. Kentucky Medical Journal, 29, 504.

Reif, S., Horgan, C. M., Hodgkin, D., Matteucci, A.-M., Creedon, T. B., \& Stewart, M. T. (2016). Access to addiction pharmacotherapy in private health plans. Journal of Substance Abuse Treatment, 66, 23-29. doi:10.1016/j.jsat.2016.03.001

Reinarman, C., \& Granfield, R. (2014). Addiction is not just a brain disease: Critical studies of addiction. In R. Granfield \& C. Reinarman (Eds.), Expanding addiction: Critical essays (pp. 1-24). New York, NY: Routledge: Taylor \& Francis Group.

Reinhardt, U. E. (2006). The pricing of U.S. hospital services: Chaos behind a veil of secrecy. Health Affairs, 25(1), 57-69. doi:10.1377/hlthaff.25.1.57

Revelle, W. (2018). Psych: Procedures for personality and psychological research. Evanston, IL: Northwestern University. Retrieved from https://CRAN.Rproject.org/package=psych

Ritchie, J., \& Spencer, L. (2002). Qualitative data analysis for applied policy research. In A. Bryman \& B. Burgess (Eds.), Analyzing qualitative data (pp. 173-194). London, England; New York, NY: Routledge: Taylor \& Francis Group.

Rogers, E. M. (2003). The diffusion of innovation (5th ed.). New York, NY: Free Press.

Ronan, M. V., \& Herzig, S. J. (2016). Hospitalizations related to opioid abuse/dependence and associated serious infections increased sharply, 2002-12. Health Affairs, 35(5), 832-837. doi:10.1377/hlthaff.2015.1424

Rosenberg, C. E. (1989). Disease in history: Frames and framers. The Milbank Quarterly, Suppl 1, 1-15. doi:10.2307/3350182

Rosenthal, E. S., Karchmer, A. W., Theisen-Toupal, J., Castillo, R. A., \& Rowley, C. F. (2015). Suboptimal addiction interventions for patients hospitalized with injection drug use-associated infective endocarditis. American Journal of Medicine, 129(5), 481-485. doi:10.1016/j.amjmed.2015.09.024

Rossman, G. B., \& Wilson, B. L. (1985). Numbers and words: Combining quantitative and qualitative methods in a single large-scale evaluation study. Evaluation Review, 9(5), 627-643. doi:10.1177/0193841X8500900505

RStudio Team. (2015). RStudio: Integrated development for R. Boston, MA RStudio, Inc. Retrieved from http://www.rstudio.com/

Ruef, M., \& Scott, W. R. (1998). A multidimensional model of organizational legitimacy: Hospital survival in changing institutional environments. Administrative Science Quarterly, 43(4), 877-904. doi:10.2307/2393619

Sacco, L. N. (2014). Drug enforcement in the United States: History, policy, and trends. Retrieved from http://www.fredsakademiet.dk/ordbog/uord/drugs.pdf

Safavi, K. C., Li, S., Dharmarajan, K., Venkatesh, A., KM, S., H, L., . . Krumholz, H. (2014). Hospital variation in the use of noninvasive cardiac imaging and its association with downstream testing, interventions, and outcomes. JAMA Internal Medicine, 174(4), 546-553. doi:10.1001/jamainternmed.2013.14407

Salkever, D. S. (1976). Accessibility and the demand for preventive care. Social Science \& Medicine, 10(9-10), 469-475. doi:10.1016/0037-7856(76)90114-1 
Sallis, J. F., Owen, N., \& Fisher, E. (2015). Ecological models of health behavior. In K. Glanz, B. K. Rimer, \& K. Viswanath (Eds.), Health behavior: Theory, research, and practice (5th ed., pp. 43-64). San Francisco, CA: Jossey-Bass Publishers.

Saloner, B., Bandara, S. N., McGinty, E. E., \& Barry, C. L. (2016). Justice-involved adults with substance use disorders: Coverage increased but rates of treatment did not in 2014. Health Affairs, 35(6), 1058-1066. doi:10.1377/hlthaff.2016.0005

Saloner, B., \& Cook, B. L. (2013). Blacks and Hispanics are less likely than whites to complete addiction treatment, largely due to socioeconomic factors. Health Affairs, 32(1), 135-145. doi:10.1377/hlthaff.2011.0983

Saloner, B., McGinty, E. E., Beletsky, L., Bluthenthal, R., Beyrer, C., Botticelli, M., \& Sherman, S. G. (2018). A public health strategy for the opioid crisis. Public Health Reports, 133(1_suppl), 24S-34S. doi:10.1177/0033354918793627

Schmidt, D. A. (1971). Addiction in Vietnam spurs Nixon and Congress to take drastic new steps. The New York Times. Retrieved from http://query.nytimes.com/mem/archivefree/pdf?res=9E03E0DF1330E73BBC4E52DFB066838A669EDE

Schneider, A. L., \& Ingram, H. (1988). Systematically pinching ideas: A comparative approach to policy design. Journal of Public Policy, 8(1), 61-80. doi:10.1017/S0143814X00006851

Schneider, A. L., \& Ingram, H. (1993). Social construction of target populations: Implications for politics and policy. American Political Science Review, 87(2), 334347. doi:10.2307/2939044

Schneider, A. L., Ingram, H., \& deLeon, P. (2014). Democratic policy design. In P. A. Sabatier \& C. M. Weible (Eds.), Theories of the Policy Process (pp. 59-103). Boulder, CO: Westview Press.

Schneider, J. (2014). Deviant drinking as disease: Alcoholism as a social accomplishment. In R. Granfield \& C. Reinarman (Eds.), Expanding addiction: Critical essays (pp. 51-61). New York, NY: Routledge: Taylor \& Francis Group.

Schuckit, M. A. (2016). Treatment of opioid-use disorders. New England Journal of Medicine, 375(4), 357-368. doi:10.1056/NEJMra1604339

Scott, W. R. (1995). Institutions and organizations. London, England: SAGE Publication.

Scott, W. R. (2003). Organizations: Rational, natural and open systems (5th ed.). Upper Saddle River, NJ: Prentice Hall, Inc.

Scott, W. R., \& Meyer, J. W. (1991). The organization of societal sectors: Propositions and early evidence. In P. J. DiMaggio \& W. W. Powell (Eds.), The new institutionalism in organizational analysis (pp. 108-142). Chicago, IL: University of Chicago Press.

Sharma, M., Lamba, W., Cauderella, A., Guimond, T. H., \& Bayoumi, A. M. (2017). Harm reduction in hospitals. Harm Reduction Journal, 14(1), 32. doi:10.1186/s12954017-0163-0 
Shengelia, B., Murray, C. J., \& Adams, O. B. (2003). Beyond access and utilization: Defining and measuring health system coverage. In C. J. Murray \& D. B. Evans (Eds.), Health systems performance assessment: Debates, methods and empiricism. (pp. 221-234). Geneva, Switzerland: World Health Organization.

Simon, H. A. (1985). Human nature in politics: The dialogue of psychology with political science. American Political Science Review, 79(2), 293-304. doi:10.2307/1956650

Skinner, J. (2011). Causes and consequences of regional variations in health care. Handbook of Health Economics, 2, 45-93. doi:10.1016/B978-0-444-535924.00002-5

Skocpol, T. (1992). Protecting soldiers and mothers (Vol. 992). Cambridge, MA: Harvard University Press.

SocioCultural Research Consultants LLC. (2016). Dedoose software.

Sordo, L., Barrio, G., Bravo, M. J., Indave, B. I., Degenhardt, L., Wiessing, L., . . PastorBarriuso, R. (2017). Mortality risk during and after opioid substitution treatment: Systematic review and meta-analysis of cohort studies. BMJ, 357, j1550. doi:10.1136/bmj.j1550

Spector, M., \& Kitsuse, J. I. (1977). Constructing social problems. Menlo Park, CA: Benjamin-Cummings Publishing Company.

Spillane, J. F. (2004). Debating the Controlled Substances Act. Drug and Alcohol Dependence, 76(1), 17-29. doi:10.1016/j.drugalcdep.2004.04.011

Spillane, J. F., \& McAllister, W. B. (2003). Keeping the lid on: A century of drug regulation and control. Drug and Alcohol Dependence, 70(3 SUPPL.), 5-12. doi:10.1016/S0376-8716(03)00096-6

StataCorp. (2017). Stata Statistical Software: Release 15. College Station, TX: StataCorp LLC.

Stevens, J. P., Wall, M. J., Novack, L., Marshall, J., Hsu, D. J., \& Howell, M. D. (2017). The critical care crisis of opioid overdoses in the United States. Annals of American Thoracic Surgery, 14(12), 1803-1809. doi:10.1513/AnnalsATS.201701-0220C

Stone, D. (2011). Policy paradox: The art of political decision making (3rd ed.). New York, NY: W. W. Norton \& Company.

Strain, E. C., \& Stoller, K. B. (1999). Introduction and historical overview. In E. C. Strain \& M. L. Stitzer (Eds.), Methadone treatment for opioid dependence (pp. 1-14). Baltimore, MD: Johns Hopkins University Press.

Stranges, E., Wier, L., Merrill, C., \& Steiner, C. (2009). Hospitalizations in which patients leave the hospital against medical advice (AMA), 2007. Retrieved from https://www.ncbi.nlm.nih.gov/books/NBK53965/

Substance Abuse and Mental Health Services Administration. (2014). Medicaid coverage and financing of medications to treat alcohol and opioid use disorders. Retrieved from https://store.samhsa.gov/product/Medicaid-Coverage-and-Financing-ofMedications-to-Treat-Alcohol-and-Opioid-Use-Disorders/sma14-4854 
Substance Abuse and Mental Health Services Administration. (2015). Certification of opioid treatment programs (OTPs). Retrieved from

https://www.samhsa.gov/medication-assisted-treatment/opioid-treatmentprograms

Substance Abuse and Mental Health Services Administration. (2016). Qualify for a physician waiver. Retrieved from https://www.samhsa.gov/medication-assistedtreatment/buprenorphine-waiver-management/qualify-for-physicianwaiver\#Physicians-in-Correctional-Facilities

Substance Abuse and Mental Health Services Administration. (2017a). SAMHSA reaffirms efforts to address the public health emergency on the opioid crisis [Press release]. Retrieved from https://www.samhsa.gov/newsroom/pressannouncements/201710260100

Substance Abuse and Mental Health Services Administration. (2017b). State targeted response to the opioid crisis grants. Retrieved from https://www.samhsa.gov/grants/grant-announcements/ti-17-014

Substance Abuse and Mental Health Services Administration. (2018). Treatment Improvement Protocol 63: Medications for opioid use disorder. Retrieved from https://store.samhsa.gov/system/files/sma18-5063fulldoc.pdf.

Surgeon General of the United States. (n.d.). Turn the tide RX. Retrieved from http://turnthetiderx.org/

Suzuki, J. (2016). Medication-assisted treatment for hospitalized patients with intravenous-drug-use related infective endocarditis. American Journal on Addictions, 25(3), 191-194. doi:10.1111/ajad.12349

Swann, J. P. (1998). FDA's origin. In G. Kurian (Ed.), A historical guide to the U.S. Government (pp. 248-254). New York, NY: Oxford University Press.

Thakarar, K., Weinstein, Z. M., \& Walley, A. Y. (2016). Optimising health and safety of people who inject drugs during transition from acute to outpatient care: Narrative review with clinical checklist. Postgraduate Medical Journal, 92(1088), 356-363. doi:10.1136/postgradmedj-2015-133720

Theisen-Toupal, J., Ronan, M. V., Moore, A., \& Rosenthal, E. S. (2017). Inpatient management of opioid use disorder: A review for hospitalists. Journal of Hospital Medicine, 12(5), 369-374. doi:10.12788/jhm.2731

Thompson, J. D. (1967). Organizations in action: Social science bases of administrative theory. New York, NY: Transaction Publishers.

Trescot, A. M., Datta, S., Lee, M., \& Hansen, H. (2008). Opioid pharmacology. Pain Physician, 11(2 Suppl), S133-153. Retrieved from https://www.painphysicianjournal.com/linkout?issn=15333159\&vol=11\&page $=$ S133

Trowbridge, P., Weinstein, Z. M., Kerensky, T., Roy, P., Regan, D., Samet, J. H., \& Walley, A. Y. (2017). Addiction consultation services - Linking hospitalized patients to outpatient addiction treatment. Journal of Substance Abuse Treatment, 79, 1-5. doi:10.1016/j.jsat.2017.05.007 
Tupper, K. W., McCrae, K., Garber, I., Lysyshyn, M., \& Wood, E. (2018). Initial results of a drug checking pilot program to detect fentanyl adulteration in a Canadian setting. Drug and Alcohol Dependence, 190, 242-245.

doi:10.1016/j.drugalcdep.2018.06.020

Turner, B. S. (1992). Regulating bodies: Essays in medical sociology (1st ed.). New York, NY: Routledge: Taylor \& Francis Group.

U.S. Census Bureau. (2015). Geography. Retrieved from https://www.census.gov/geo/reference/webatlas/regions.html

U.S. Department of Veterans Affairs. (2016a). Veterans Health Administration. Retrieved from https://www.va.gov/health/aboutVHA.asp

U.S. Department of Veterans Affairs. (2016b). VHA opioid taper decision tool. Retrieved from

https://www.pbm.va.gov/AcademicDetailingService/Documents/Pain Opioid Ta per Tool IB 10 939 P96820.pdf

U.S. Department of Veterans Affairs. (2017a). Pharmacy benefits management. Retrieved from https://www.pbm.va.gov.

U.S. Department of Veterans Affairs. (2017b). VA/DoD clinical practice guidelines: Management of substance use disorder (SUD) (2015). Retrieved from https://www.healthquality.va.gov/guidelines/mh/sud/

United Nations. (2013). The International Drug Control Conventions: Single Convention on Narcotic Drugs of 1961 as amended by the 1972 Protocol, Convention on Psychotropic Substances of 1971, United Nations Convention against Illicit Traffic in Narcotic Drugs and Psychotropic Substances of 1988. New York, NY Retrieved from

https://www.unodc.org/documents/commissions/CND/Int Drug_Control_Conve ntions/Ebook/The International_Drug Control Conventions E.pdf.

United Nations Office on Drugs and Crime. (2016). World drug report 2016. Retrieved from https://www.unodc.org/doc/wdr2016/WORLD DRUG REPORT 2016 web.pdf

United States Department of Health \& Human Services. (2016). Facing addiction in America: The Surgeon General's report on alcohol, drugs, and health. Retrieved from https://addiction.surgeongeneral.gov/surgeon-generals-report.pdf

University of Wisconsin. (2017). Opioid consumption maps - ME minus methadone, $\mathrm{mg} / \mathrm{capita}, 2015$. Retrieved from https://ppsg.medicine.wisc.edu/

VA Office of Inspector General. (2012). Independent review of VA's fiscal year 2011 detailed accounting summary report to the Office of National Drug Control Policy. Retrieved from https://www.va.gov/oig/pubs/VAOIG-12-01071-122.pdf.

Valverde, M. (1998). Diseases of the will: Alcohol and the dilemmas of freedom. Cambridge, MA: Cambridge University Press. 
Velez, C. M., Nicolaidis, C., Korthuis, P. T., \& Englander, H. (2017). "It's been an experience, a life learning experience": A qualitative study of hospitalized patients with substance use disorders. Journal of General Internal Medicine, 32(3), 296-303. doi:10.1007/s11606-016-3919-4

Vincent, B. M., Wiitala, W. L., Burns, J. A., Iwashyna, T. J., \& Prescott, H. C. (2018). Using Veterans Affairs Corporate Data Warehouse to identify 30-day hospital readmissions. Health Services and Outcomes Research Methodology, 18(3), 143154. doi:doi: 10.1007/s10742-018-0178-3

Volkow, N. D., \& Collins, F. S. (2017). The role of science in addressing the opioid crisis. New England Journal of Medicine, 377(4), 391-394. doi:10.1056/NEJMsr1706626

Volkow, N. D., Koob, G. F., \& McLellan, A. T. (2016). Neurobiologic advances from the brain disease model of addiction. New England Journal of Medicine, 374(4), 363371. doi:10.1056/NEJMra1511480

Wakeman, S. E., Metlay, J. P., Chang, Y., Herman, G. E., \& Rigotti, N. A. (2017). Inpatient addiction consultation for hospitalized patients increases post-discharge abstinence and reduces addiction severity. Journal of General Internal Medicine, 32(8), 909-916. doi:10.1007/s11606-017-4077-z

Warner, M., Trinidad, J. P., Bastian, B. A., Miniño, A. M., \& Hedegaard, H. (2016). Drugs most frequently involved in drug overdose deaths: United States, 2010-2014. Retrieved from https://www.cdc.gov/nchs/data/nvsr/nvsr65/nvsr65 10.pdf

Wasey, J. O. (2018). icd: Comorbidity calculations and tools for ICD-9 and ICD-10 codes. Retrieved from https://CRAN.R-project.org/package=icd

WBUR News \& Wire Services. (2018). Lawmakers send opioid bill to Baker's desk. Retrieved from https://www.wbur.org/commonhealth/2018/08/01/opioidlegislation-to-governor

Weeks, W. B., \& West, A. N. (2018). Veterans health administration hospitals outperform non-veterans health administration hospitals in most health care markets. Annals of Internal Medicine, Advance online publication. doi:10.7326/M18-1540

Weick, K. E. (1995). Sensemaking in organizations (1st ed.). Thousand Oaks, CA: SAGE Publications.

Weinstein, Z. M., Wakeman, S. E., \& Nolan, S. (2018). Inpatient addiction consult service: Expertise for hospitalized patients with complex addiction problems. Medical Clinics of North America, 102(4), 587-601. doi:10.1016/j.mcna.2018.03.001

Weiss, A. J., Bailey, M. K., O’Malley, L., Barret, M. L., Elixhauser, A., \& Steiner, C. A. (2017a). HCUP statistical brief \#224: Patient characteristics of opioid-related inpatient stays and emergency department visits nationally and by state, 2014. Retrieved from https://www.hcup-us.ahrq.gov/reports/statbriefs/sb224-PatientCharacteristics-Opioid-Hospital-Stays-ED-Visits-by-State.pdf 
Weiss, A. J., Bailey, M. K., O'Malley, L., Barret, M. L., Elixhauser, A., \& Steiner, C. A. (2017b). HCUP statistical brief \#226: Patient residence characteristics of opioidrelated inpatient stays and emergency department visits nationally and by state, 2014. Retrieved from https://www.hcup-us.ahrq.gov/reports/statbriefs/sb226Patient-Residence-Opioid-Hospital-Stays-ED-Visits-by-State.pdf

Weiss, A. J., \& Elixhauser, A. (2014). HCUP statistical brief \#180: Overview of hospital stays in the United States, 2012. Retrieved from https://www.hcupus.ahrq.gov/reports/statbriefs/sb180-Hospitalizations-United-States-2012.pdf

Weiss, A. J., Elixhauser, A., Barret, M. L., Steiner, C. A., Bailey, M. K., \& O’Malley, L. (2016). HCUP statistical brief \#219: Opioid-related inpatient stays and emergency department visits by state, 2009-2014. Retrieved from https://www.hcupus.ahrq.gov/reports/statbriefs/sb219-Opioid-Hospital-Stays-ED-Visits-byState.pdf

Weiss, A. J., \& Heslin, K. C. (2018). HCUP statistical brief \#239: Payers of opioid-related inpatient stays and emergency department visits nationally and by state, 2010 and 2015. Retrieved from https://www.hcupus.ahrq.gov/reports/statbriefs/sb239-Opioid-Payer-Hospital-Stays-ED-Visits-byState.pdf

Wennberg, J. E., Fisher, E. S., \& Skinner, J. S. (2002). Geography and the debate over Medicare reform. Health Affairs, Suppl Web Exclusives, W96-114. doi:10.1377/hlthaff.w2.96.

Wesson, D. R., \& Ling, W. (2003). The clinical opiate withdrawal scale (COWS). Journal of Psychoactive Drugs, 35(2), 253-259. doi:10.1080/02791072.2003.10400007

White House. (2017). President's commission on combating drug addiction and the opioid crisis. Retrieved from https://www.whitehouse.gov/ondcp/presidentscommission

White, W. L. (2002). Trick or treat? A century of American responses to heroin addiction. In D. F. Musto, P. Korsmeyer, \& T. W. Maulucci (Eds.), One hundred years of heroin (pp. 131-148). Westport, CT: Greenwood Publishing Group, Inc.

Wickham, H. (2011). plyr: The split-apply-combine strategy for data analysis. Journal of Statistical Software, 40(1). doi:10.18637/jss.v040.i01

Wickham, H. (2017). tidyverse: Easily install and load the 'Tidyverse'. . Retrieved from https://CRAN.R-project.org/package=tidyverse

Williamson, O. E. (1979). Transaction-cost economics: The governance of contractual relations. The Journal of Law and Economics, 22(2), 233-261. doi:10.1086/466942

Williamson, O. E. (1985). The economic intstitutions of capitalism. New York, NY: Simon and Schuster.

Williamson, O. E. (1994). Transaction cost economics and organization theory. In N. Smelser \& R. Swedberg (Eds.), Handbook of economic sociology (pp. 77-107). Princeton, NJ: Princeton University Press. 
Williamson, O. E., \& Ouchi, W. G. (1981). The markets and hierarchies and visible hand perspectives. In A. Van de Ven \& W. F. Joyce (Eds.), Perspectives on Organization Design and Behavior (pp. 347-370). New York, NY: John Wiley \& Sons, Inc.

World Health Organization. (2009). Guidelines for the psychosocially assisted pharmacological treatment of opioid dependence (9789241547543). Retrieved from http://www.who.int/substance abuse/publications/opioid dependence guideli nes.pdf

World Health Organization. (2017). WHO model list of essential medicines: 20th list. Retrieved from

http://www.who.int/medicines/publications/essentialmedicines/20th EML2017. pdf?ua=1

Wyse, J. J., Gordon, A. J., Dobscha, S. K., Morasco, B. J., Tiffany, E., Drexler, K., . . . Lovejoy, T. I. (2018). Medications for opioid use disorder in the Department of Veterans Affairs (VA) health care system: Historical perspective, lessons learned, and next steps. Substance Abuse, 39(2), 139-144. doi:10.1080/08897077.2018.1452327

Yablonsky, L. (1967). Synanon: The tunnel back. Baltimore, MD: Penguin Books.

Zeckhauser, R. J., \& Pratt, J. W. (1985). Principals and agents: The structure of business. Cambridge, MA: Harvard Business School Press Boston.

Zhou, C., Florence, C. S., \& Dowell, D. (2016). Payments for opioids shifted substantially to public and private insurers while consumer spending declined, 1999-2012. Health Affairs, 35(5), 824-831. doi:10.1377/hlthaff.2015.1103 


\section{Appendix A: The DSM 5 Opioid Use Disorder Diagnostic Criteria}

From the American Psychiatric Association (2013):

"A problematic pattern of opioid use leading to clinically significant impairment or distress, as manifested by at least two of the following, occurring within a 12-month period:

1. Opioids are often taken in larger amounts or over a longer period than was intended.

2. There is a persistent desire or unsuccessful efforts to cut down or control opioid use.

3. A great deal of time is spent in activities necessary to obtain the opioid, use the opioid, or recover from its effects.

4. Craving, or a strong desire or urge to use opioids.

5. Recurrent opioid use resulting in a failure to fulfill major role obligations at work, school, or home.

6. Continued opioid use despite having persistent or recurrent social or interpersonal problems caused or exacerbated by the effects of opioids.

7. Important social, occupational, or recreational activities are given up or reduced because of opioid use.

8. Recurrent opioid use in situations in which it is physically hazardous.

9. Continued opioid use despite knowledge of having a persistent or recurrent physical or psychological problem that is likely to have been caused or exacerbated by the substance.

10. Tolerance, as defined by either of the following: A need for markedly increased amounts of opioids to achieve intoxication or desired effect; or a markedly diminished effect with continued use of the same amount of an opioid. * This criterion is not considered to be met for those taking opioids solely under appropriate medical supervision.

11. Withdrawal, as manifested by either of the following: The characteristic opioid withdrawal syndrome (refer to Criteria A and B of the criteria set for opioid withdrawal, pp. 547-548); or opioids (or a closely related substance) are taken to relieve or avoid withdrawal symptoms. ${ }^{*}$ This criterion is not considered to be met for those individuals taking opioids solely under appropriate medical supervision.

Specify if:

In early remission: After full criteria for opioid use disorder were previously met, none of the criteria for opioid use disorder have been met for at least 3 months but for less than 12 months (with the exception that Criterion A4, "Craving, or a strong desire or urge to use opioids," may be met).

In sustained remission: After full criteria for opioid use disorder were previously met, none of the criteria for opioid use disorder have been met at any time during a period of 12 months or longer (with the exception that Criterion A4, "Craving, or a strong desire or urge to use opioids," may be met).

On maintenance therapy: This additional specifier is used if the individual is taking a prescribed agonist medication such as methadone or buprenorphine and none of the criteria for opioid use 
disorder have been met for that class of medication (except tolerance to, or withdrawal from, the agonist). This category also applies to those individuals being maintained on a partial agonist, an agonist/antagonist, or a full antagonist such as oral naltrexone or depot naltrexone.

In a controlled environment: This additional specifier is used if the individual is in an environment where access to opioids is restricted.

Coding based on current severity: Note for ICD-10-CM codes: If an opioid intoxication, opioid withdrawal, or another opioid-induced mental disorder is also present, do not use the codes below for opioid use disorder. Instead, the comorbid opioid use disorder is indicated in the 4th character of the opioid-induced disorder code (see the coding note for opioid intoxication, opioid withdrawal, or a specific opioid-induced mental disorder). For example, if there is comorbid opioid-induced depressive disorder and opioid use disorder, only the opioid-induced depressive disorder code is given, with the 4th character indicating whether the comorbid opioid use disorder is mild, moderate, or severe: F11.14 for mild opioid use disorder with opioidinduced depressive disorder or F11.24 for a moderate or severe opioid use disorder with opioidinduced depressive disorder.

305.50 (F11.10) Mild: Presence of 2-3 symptoms.

304.00 (F11.20) Moderate: Presence of 4-5 symptoms.

304.00 (F11.20) Severe: Presence of 6 or more symptoms."

(American Psychiatric Association, 2013) 


\section{Appendix B: OHSU IRB Approved Informational Sheet}

TITLE: Opioid Agonist Treatment in Hospitals

PRINCIPAL INVESTIGATOR: Dennis McCarty, PhD (503) 494-1177

CO-INVESTIGATOR: Kelsey Priest, MPH

FUNDED BY: National Institutes of Health (NIH), National Institute on Drug Abuse (NIDA)

PURPOSE:

You have been invited to be in this research study because you are either a national hospital addiction medicine expert (e.g., physician, pharmacist, nurse, social worker, hospital administrator, health services researcher, or government official), a member of a hospital pharmacy and therapeutics committee for at least 6 months (e.g., nursing, physician, pharmacist, hospital administrator), or a health professional (e.g., nursing, physician, pharmacist, hospital administrator) who works on a hospital addiction consult service.

The purpose of this interview is to explore your perspectives about your organization's hospital opioid use disorder care delivery policies, procedures, and practice, specifically:

1. Hospital OUD care delivery policies, procedures, and practice.

2. Barriers and facilitators to hospital OUD treatment and OAT delivery.

3. Ideas or solutions for enhancing hospital OUD treatment and OAT delivery.

\section{PROCEDURES:}

This study requires one 45-to 60-minute telephone (or in-person) audio recorded interview. If you mention a specific hospital policy during the course of the interview I will request a copy and I may follow-up with clarifying questions.

We are recruiting approximately 30 key informants to participate in this study who are professionals identified from across the United States as hospital-based OUD treatment experts.

The recordings of the interviews will be professionally transcribed (meaning that what is said will be written down exactly as stated), reviewed, summarized, and entered into a software program that is designed to facilitate analysis of the interviews. Notes will be taken during the interviews and entered into the same software program. All data from the interviews will be deidentified and coded with a special number when entered and stored in the software program.

If you have any questions, concerns or complaints regarding this study now or in the future, or you think you may have been injured or harmed by the study, contact Dr. Dennis McCarty (503) 494-1177 or mccartyd@ohsu.edu. 


\section{RISKS:}

Although we have made every effort to protect your identity, there is a minimal risk of loss of confidentiality. The recording of the interview will be stored on OHSU encrypted and passwordprotected servers for no longer than 3 years after study completion. Access to the data will be granted only to the study researchers. All of the data kept in notes, recordings, and transcriptions related to the research will be destroyed as soon as the research is complete.

\section{BENEFITS:}

You will not benefit from being in this study. However, by serving as a participant, you may help us learn how to benefit patients with opioid use disorder in the future.

\section{CONFIDENTIALITY:}

We will take steps to keep your personal information confidential, but we cannot guarantee total privacy. All NIDA sponsored studies have an automatic federal certificate of confidentiality to help protect the confidentiality of the data.

\section{PARTICIPATION:}

This research is being overseen by an Institutional Review Board ("IRB"). You may talk to the OHSU IRB at (503) 494-7887 or irb@ohsu.edu if:

- Your questions, concerns, or complaints are not being answered by the research team.

- You want to talk to someone besides the research team.

- You have questions about your rights as a research subject.

- You want to get more information or provide input about this research.

You may also submit a report to the OHSU Integrity Hotline online at https://secure.ethicspoint.com/domain/media/en/gui/18915/index.html or by calling toll-free (877) 733-8313 (anonymous and available 24 hours a day, 7 days a week).

You do not have to join this or any research study. If you do join, and later change your mind, you may quit at any time. If you refuse to join, or withdraw early from the study, there will be no penalty or loss of any benefits to which you are otherwise entitled. 


\section{Appendix C: OHSU IRB Approved Email Recruitment Script}

Subject Line: OHSU Research Study: Opportunity for Participation

\section{Email Primary Contact}

"Dear

My name is Kelsey Priest, and I am a fourth year MD/PhD student at Oregon Health \& Science University (OHSU) and a doctoral student at OHSU-Portland State University School of Public Health in the Health Systems and Policy program. I am contacting you in connection with a research study (OHSU IRB approval \#STUDY00018092) that I am conducting as part of my dissertation, under the supervision of Dr. Dennis McCarty who is the chair of my dissertation committee. This project seeks to learn more about the health care service patterns of opioid agonist therapy (OAT) delivery in the hospital setting for persons with opioid use disorder.

I am seeking to speak with an expert from your hospital on hospital-based practices and policies related to the treatment of persons with opioid use disorder. Preferably this will be someone who has worked on opioid use disorder related initiatives for several years and/or has seen the implementation of programs, like an addiction consult services over time. The purpose of the interview is to capture this expert's perspectives about your organization's hospital opioid use disorder care delivery policies, procedures, and practice, specifically:

1. Hospital OUD care delivery policies, procedures, and practice.

2. Barriers and facilitators to hospital OUD treatment and OAT delivery.

3. Ideas or solutions for enhancing hospital OUD treatment and OAT delivery.

I am emailing to see if you would be interested in participating as a key informant in our research study or if you could refer me to someone at your hospital who you think would be better able to answer these questions.

In either case, please respond to this email with either your recommended contact or indicate whether or not you are interested in participating.

If you are interested in participating, I will arrange a time over email for us to have a 45 to 60minute confidential phone interview (or in-person meeting) to talk at your convenience.

If you are recommending a colleague, I will reach out to them directly.

If you have concerns about this research please contact Dr. Dennis McCarty at (503) 494-1177 and mccartyd@ohsu.edu.If you have questions about your rights as a research subject or research-related injuries, you can call the OHSU Research Integrity Office at 503-494-7887.

\section{Email Secondary Contact}

“Dear

My name is Kelsey Priest, and I am a fourth year MD/PhD student at Oregon Health\& Science University (OHSU) and a doctoral student at OHSU-Portland State University School of Public Health in the Health Systems and Policy program. I am contacting you in connection with a 
research study (OHSU IRB approval \#STUDY00018092) that I am conducting as part of my dissertation, under the supervision of Dr. Dennis McCarty who is the chair of my dissertation committee. This project seeks to learn more about the health care service patterns of opioid agonist therapy (OAT) delivery in the hospital setting for persons with opioid use disorder.

I am seeking to speak with an expert from your hospital on hospital-based practices and policies related to the treatment of persons with opioid use disorder. Preferably this will be someone who has worked on opioid use disorder related initiatives for several years and/or has seen the implementation of programs, like an addiction consult services over time. The purpose of the interview is to capture this expert's perspectives about your organization's hospital opioid use disorder care delivery policies, procedures, and practice, specifically:

1. Hospital OUD care delivery policies, procedures, and practice.

2. Barriers and facilitators to hospital OUD treatment and OAT delivery.

3. Ideas or solutions for enhancing hospital OUD treatment and OAT delivery.

You have been identified by [references name] as an expert in hospital-based practices and policies related to the treatment of persons with opioid use disorder at your hospital.

I am emailing to see if you would be interested in participating as a key informant in our research study. If you are interested please respond to this email, and I will arrange a time for us to have a 45 to 60 -minute confidential phone interview (or in-person meeting) to talk at your convenience.

If you have concerns about this research please contact Dr. Dennis McCarty at (503) 494-1177 and mccartyd@ohsu.edu.

If you have questions about your rights as a research subject or research-related injuries, you can call the OHSU Research Integrity Office at 503-494-7887. 


\section{Appendix D: OHSU IRB Approved Key Informant Demographic Survey}

What is your age?

- $<25$

- $25-29$

- $30-34$

- $\quad 35-39$

- $40-44$

- $45-49$

- $50-54$

- 55-59

- 60-64

- 65-69

- 70 or older

$\bullet$

With what gender do you identity?

- Woman

- Man

- Other

- Decline to Answer

How do you racially identify?

- American Indian/Alaska Native

- Asian

- Native Hawaiian or Other Pacific Islander

- Black or African American

- White

- More than one race

- Decline to Answer

What ethnicity best describes you? [pick one]

- Hispanic or Latino

- Not Hispanic or Latino

- Decline to Answer

What health professional degree(s) do you have? And what year did you graduate from each $\operatorname{program}(\mathrm{s})$ ?

Which profession best describes your current work?

- Hospital administrator/manager

- Nurse

- Pharmacist

- Physician

○ Specialty: 
- Addiction Medicine Board Certification? $(\mathrm{y} / \mathrm{n})$

- Social worker

- Behavioral health specialist

- Legal counsel

- Other

For how many years have you worked at your respective hospital?

- $<1$

- $1-2$

- 3-5

- more than 5

For how many years have you been the fellowship program director?

- $<1$

- $1-2$

- 3-5

- more than 5

For how many years have you worked on the addiction consult service (if applicable)?

- $<1$

- $1-2$

- 3-5

- more than 5

Does your hospital have methadone on the formulary for treatment of OUD?

- Yes

- No

- Unknown

Does your hospital have buprenorphine on the formulary for treatment of OUD?

- Yes

- No

- Unknown 


\section{Appendix E: OHSU IRB Approved Semi-Structured Interview Guide}

ID \#:

Date:

\section{Introduction}

My name is Kelsey Priest, and I am a fourth year MD/PhD student at Oregon Health \& Science University (OHSU) and a doctoral student at OHSU-Portland State University School of Public Health in the Health Systems and Policy program. As part of the requirements of the doctoral program, I am conducting dissertation research to study the treatment of persons with opioid use disorder (OUD) in the hospital setting. As the [position of research participant] for the [hospital name], you have been identified as knowledgeable about the treatment of persons with opioid use disorder at [hospital name].

This project is an opportunity to learn more about the health care service patterns of opioid agonist therapy (OAT) delivery in the hospital setting for persons with opioid use disorder. The purpose of this interview is to capture your perspectives about your organization's hospital opioid use disorder care delivery policies, procedures, and practice, specifically:

1. Hospital OUD care delivery policies, procedures, and practice.

2. Barriers and facilitators to hospital OUD treatment and OAT delivery.

3. Ideas or solutions for enhancing hospital OUD treatment and OAT delivery.

The interview will last between 45 and 60 minutes, depending on the length of your answers. If you describe any hospital-based policies during the interview I will follow-up via email after the interview with a request to see them with your permission and I may also follow up with additional clarifying questions.

\section{Consent}

I have provided the consent form ahead of time, and I want to ensure that you are clear on the expectations of participation. I assume that your presence here today indicates you have read the consent form. Do you have any questions about your participation in this research study? I would now like to receive verbal consent to participate from you.

\section{$<$ Obtain verbal consent $>$}

\section{Audio Recording Instructions}

With your permission, I will take notes and record the interview. Your participation in this interview is voluntary; you do not have to answer any question that you do not want to answer and you may stop the interview at any time. All individual responses will be kept confidential.

The recording and my notes will help me to accurately represent our discussion; no one else will ever hear the recordings or see the written transcripts. If there are things that you tell me that you do not wish repeated, please indicate this so that I do not include those comments in any summaries or reports that I develop from this interview. 
Similarly, if at any time you would like me to stop recording, please indicate this and I will turn off the recorder. Findings will be reported in the aggregate with larger themes identified within and across hospitals. Quotes will be selected to illustrate these broader themes and will be presented without attribution to individuals. Do I have your permission to record this interview?

$<$ Obtain verbal consent, and turn on the recording device>

At this time, do you have any other questions or concerns?

$<$ After addressing any questions and/or concerns $>$

Then let us begin.

\section{Questions}

1. We will start with a brief demographic survey.

<Reads questions from key informant demographic survey>

2. Role and Introductions. Please tell me briefly about your role at your institution and within the hospital.

3. Responsibility of Care. Who (what service) within the hospital is responsible for the clinical management of an OUD during hospitalization?

a. Probe: Dependent on clinical scenario (e.g., surgical vs. non-surgical or acuity).

4. Care Delivery Mechanisms. Does your hospital have an addiction consult service?

a. If yes, when did the service start? What was the context or catalyst for this change?

i. Probe: Since that time, what shifts in culture and/or stigma of persons with OUD or other addictive disorders have occurred?

ii. Probe: How have hospital policies, procedures, or guidelines changed over time related to care for persons with OUD since the establishment of the service?

iii. Probe: What were the facilitators for starting the service?

1. Probe: Within the organization?

2. Probe: Outside the organization?

iv. Probe: What were the barriers to starting the service?

1. Probe: Within the organization?

2. Probe: Outside the organization?

b. If no, is this something that is being considered? If not, why do you think that is?

5. Addiction Consult Service Structure and Design. What is the current design of the addiction consult service?

a. Probe: Describe the team composition (e.g., what professionals are on the team). 
b. Probe: Describe the services provided by the consult service (e.g., medication management, pain consultation, harm reduction).

c. Probe: Describe the availability of the service (e.g., weekdays only, 24-7 coverage).

6. Current Policies. What are your hospital's current policies and procedures (e.g., guidelines) for OUD management and/or withdrawal?

a. Probe: Specifically, does your hospital have policies or procedures on the continuation of methadone or buprenorphine for OUD treatment during hospitalization?

i. If so, please describe the policies.

ii. Have these changed over time? If yes, how so?

b. Probe: Does your hospital have any policies or procedures on the induction of methadone or buprenorphine during hospitalization?

i. If so, please describe the policies

ii. Have these changed over time? If yes, how so?

c. Probe: Does your hospital have any policies or procedures on the use of buprenorphine or methadone to manage withdrawal?

i. If so, please describe the policies.

ii. Have these changed over time? If yes, how so?

d. Probe: If applicable, do these policies or procedures reflect practice generally?

7. Organizational Barriers. What do you think are some of the organizational barriers to implementing policies and procedures for caring for persons with OUD at your hospital?

a. Probe: Physical environment/location?

b. Probe: Leadership?

c. Probe: Resources and staffing?

d. Probe: Policy implementation process?

e. Probe: Hospital culture and understanding of OUD?

8. External Barriers. What do you think are some of the external barriers to implementing policies and procedures for caring for persons with OUD at your hospital?

a. Probe: Federal or local policies? (e.g., federal regulations or insurance regulations)

b. Probe: System service delivery issues (e.g., care transitions)?

c. Probe: Other local hospitals?

9. Organizational Facilitators. What do you think are some of the organizational facilitators for implementing policies and procedures for caring for persons with opioid use disorder at your hospital?
a. Probe: Physical environment/location?
b. Probe: Leadership?
c. Probe: Resources and staffing?
d. Probe: Policy implementation process?
e. Probe: Hospital culture and understanding of OUD? 
10. External Facilitators. What do you think are some of the external facilitators for implementing policies and procedures for caring for persons with opioid use disorder at your hospital?
a. Probe: Federal or local policies?
b. Probe: System?
c. Probe: Other local hospitals?

11. Is there anything else that we have not discussed about the treatment of OUD in your hospital?

\section{Closing the Interview}

Thank you for participating in this interview, and for your thoughtful comments, insights and candor. I am meeting with approximately 19 other key informants from other hospitals across the United States. I will be analyzing and synthesizing the key themes and issues that emerge over the course of the study. If you think of anything else, please contact me. Findings from the interviews will be included in my dissertation and read by the OHSU-PSU School of Public Health faculty serving on my dissertation committee. At the conclusion of the study, I would be happy to share a report of the aggregated findings with you. May I contact you if I have any follow up questions? Again, thank you for your time and willingness to participate. 
Appendix F: Final Qualitative Analyses Codebook \& Code Distribution

\begin{tabular}{|c|c|}
\hline Category (code) & $\begin{array}{l}\text { Code } \\
\text { Count }\end{array}$ \\
\hline 1. Addiction Consult Service: Elements related to AMC service & 239 \\
\hline 2. Culture and Practice Shift & 9 \\
\hline 3. Design & 44 \\
\hline 4. Historical Context & 77 \\
\hline 5. Policy Influence & 2 \\
\hline 6. Team Composition & 32 \\
\hline 7. Care Delivery Topics: Elements related to OUD treatment within hospital & 217 \\
\hline 8. Criminal Justice & 5 \\
\hline 9. Culture Change & 8 \\
\hline 10. Education & 34 \\
\hline 11. Electronic Health Record & 8 \\
\hline 12. Emergency Department & 30 \\
\hline 13. Endocarditis & 11 \\
\hline 14. Formulary & 20 \\
\hline 15. Harm Reduction & 12 \\
\hline 16. Injectable Naltrexone & 2 \\
\hline 17. Linkage to Care & 63 \\
\hline 18. Naloxone & 11 \\
\hline 19. Non-evidence-based care & 24 \\
\hline 20. Nursing involvement/initiative & 10 \\
\hline 21. Pain Consult & 12 \\
\hline 22. Peers & 19 \\
\hline 23. Psychiatry Consult & 36 \\
\hline 24. Script on DX & 8 \\
\hline 25. Security and Public Safety & 5 \\
\hline 26. Stigma & 43 \\
\hline 27. Stimulant Use & 5 \\
\hline 28. Tobacco/Nicotine & 4 \\
\hline 29. Trainees & 61 \\
\hline 30. Trauma & 2 \\
\hline 31. Demand-Side Attributes: Elements related to patient characteristics & 27 \\
\hline 32. Patient Characteristics & 29 \\
\hline $\begin{array}{l}\text { 33. External Environment: Elements external to the hospital influencing care } \\
\text { delivery }\end{array}$ & 66 \\
\hline 34. External Treatment Organizations & 49 \\
\hline 35. Media Coverage & 12 \\
\hline 36. Normative social structure & 12 \\
\hline 37. Policies and Regulations & 19 \\
\hline 38. Politics and Political Leadership & 24 \\
\hline 39. Quality Metrics & 9 \\
\hline 40. Hospital Policy: Policy and practice related to the treatment of OUD & 205 \\
\hline 41. Aberrant Drug Use/Behavioral Agreement & 25 \\
\hline 42. BYOD & 1 \\
\hline 43. Induction & 43 \\
\hline 44. OAT continuation & 23 \\
\hline 45. PICC & 17 \\
\hline
\end{tabular}




\begin{tabular}{|c|c|}
\hline 46. Pain Management & 24 \\
\hline 47. Policy Frame & 5 \\
\hline 48. Policy Process & 22 \\
\hline 49. Pregnancy & 16 \\
\hline 50. Security & 6 \\
\hline 51. Withdrawal Management & 47 \\
\hline $\begin{array}{l}\text { 52. Hospital Supply-Side Attributes: Elements within the hospital influencing } \\
\text { care }\end{array}$ & 92 \\
\hline 53. Leadership & 44 \\
\hline 54. Location/Geography & 3 \\
\hline 55. Misunderstanding of Policy & 13 \\
\hline 56. Normative Social Structures & 18 \\
\hline 57. Research, Evaluation, Data & 22 \\
\hline 58. Resources and Staffing & 71 \\
\hline 59. Stakeholders & 8 \\
\hline 60. Other Models of Care & 62 \\
\hline 61. Ad Hoc Consults & 13 \\
\hline 62. Bridge Clinic & 6 \\
\hline 63. Detox & 20 \\
\hline 64. Longitudinal Care (within the same institution) & 4 \\
\hline 65. OTP & 33 \\
\hline 66. Service or Policy Design Lever & 21 \\
\hline $\begin{array}{l}\text { 67. Financing: Elements related to financing care and delivery of services for } \\
\text { persons with OUD }\end{array}$ & 97 \\
\hline
\end{tabular}




\section{Appendix G: RStudio Packages}

dunn.test

Dinno, A. (2017). Dunn.test: Dunn's Test of multiple comparisons using rank sums. Retrieved from https://CRAN.R-project.org/package=dunn.test

car

Fox, J., \& Weisberg, S. (2011). Car. Thousand Oaks, CA: SAGE Publications. Retrieved from http://socserv.socsci.mcmaster.ca/ifox/Books/Companion

psych

Revelle, W. (2018). Psych: Procedures for personality and psychological research. Evanston, IL: Northwestern University. Retrieved from https://CRAN.R-

project.org/package=psych

icd

Wasey, J. O. (2018). icd: Comorbidity calculations and tools for ICD-9 and ICD-10 codes. Retrieved from https://CRAN.R-project.org/package=icd

plyr

Wickham, H. (2011). plyr: The split-apply-combine strategy for data analysis. Journal of Statistical Software, 40(1). doi:10.18637/jss.v040.i01

tidyverse

Wickham, H. (2017). tidyverse: Easily install and load the 'Tidyverse.'Retrieved from https://CRAN.R-project.org/package=tidyverse 
Appendix H: OUD Diagnosis Codes Queried

\begin{tabular}{|c|c|}
\hline $\begin{array}{l}\text { ICD-10-CM } \\
\text { Code }\end{array}$ & ICD-10-CM Description \\
\hline F11.10 & Opioid abuse, uncomplicated \\
\hline F11.11 & Opioid abuse, in remission \\
\hline F11.120 & Opioid abuse with intoxication, uncomplicated \\
\hline F11.121 & Opioid abuse with intoxication delirium \\
\hline F11.122 & Opioid abuse with intoxication with perceptual disturbance \\
\hline F11.129 & Opioid abuse with intoxication, unspecified \\
\hline F11.14 & Opioid abuse with opioid-induced mood disorder \\
\hline F11.150 & Opioid abuse with opioid-induced psychotic disorder with delusions \\
\hline F11.151 & Opioid abuse with opioid-induced psychotic disorder with hallucinations \\
\hline F11.159 & Opioid abuse with opioid-induced psychotic disorder, unspecified \\
\hline F11.181 & Opioid abuse with opioid-induced sexual dysfunction \\
\hline F11.182 & Opioid abuse with opioid-induced sleep disorder \\
\hline F11.188 & Opioid abuse with other opioid-induced disorder \\
\hline F11.19 & Opioid abuse with unspecified opioid-induced disorder \\
\hline F11.20 & Opioid dependence, uncomplicated \\
\hline F11.220 & Opioid dependence with intoxication, uncomplicated \\
\hline F11.21 & Opioid dependence, in remission \\
\hline F11.221 & Opioid dependence with intoxication delirium \\
\hline F11.222 & Opioid dependence with intoxication with perceptual disturbance \\
\hline F11.229 & Opioid dependence with intoxication, unspecified \\
\hline F11.23 & Opioid dependence with withdrawal \\
\hline F11.24 & Opioid dependence with opioid-induced mood disorder \\
\hline F11.250 & Opioid dependence with opioid-induced psychotic disorder with delusions \\
\hline F11.251 & Opioid dependence with opioid-induced psychotic disorder with hallucinations \\
\hline F11.259 & Opioid dependence with opioid-induced psychotic disorder, unspecified \\
\hline F11.281 & Opioid dependence with opioid-induced sexual dysfunction \\
\hline F11.282 & Opioid dependence with opioid-induced sleep disorder \\
\hline F11.288 & Opioid dependence with other opioid-induced disorder \\
\hline F11.29 & Opioid dependence with unspecified opioid-induced disorder \\
\hline F11.90 & Opioid use, unspecified, uncomplicated \\
\hline F11.920 & Opioid use, unspecified, with intoxication, uncomplicated \\
\hline F11.921 & Opioid use, unspecified, with intoxication delirium \\
\hline F11.922 & Opioid use, unspecified, with intoxication with perceptual disturbance \\
\hline F11.929 & Opioid use, unspecified, with intoxication, unspecified \\
\hline F11.93 & Opioid use, unspecified with withdrawal \\
\hline F11.94 & Opioid use, unspecified with opioid-induced mood disorder \\
\hline F11.950 & Opioid use, unspecified with opioid-induced psychotic disorder with delusions \\
\hline F11.951 & $\begin{array}{l}\text { Opioid use, unspecified with opioid-induced psychotic disorder with } \\
\text { hallucinations }\end{array}$ \\
\hline F11.959 & Opioid use, unspecified with opioid-induced psychotic disorder, unspecified \\
\hline
\end{tabular}




\begin{tabular}{|c|c|}
\hline F11.981 & Opioid use, unspecified with opioid-induced sexual dysfunction \\
\hline F11.982 & Opioid use, unspecified with opioid-induced sleep disorder \\
\hline F11.988 & Opioid use, unspecified with other opioid-induced \\
\hline F11.99 & Opioid use, unspecified with unspecified opioid-induced disorder \\
\hline T40.0X1A & Poisoning by opium, accidental (unintentional), initial encounter \\
\hline T40.0X1D & Poisoning by opium, accidental (unintentional), subsequent encounter \\
\hline $\mathrm{T} 40.0 \times 1 \mathrm{~S}$ & Poisoning by opium, accidental (unintentional), sequelae \\
\hline T40.0X4A & Poisoning by opium, undetermined, initial encounter \\
\hline T40.0X4D & Poisoning by opium, undetermined, subsequent encounter \\
\hline $\mathrm{T} 40.0 \times 4 \mathrm{~S}$ & Poisoning by opium, undetermined, sequelae \\
\hline $\mathrm{T} 40.0 \times 5 \mathrm{~A}$ & Adverse effect of opium, initial encounter \\
\hline T40.0X5D & Adverse effect of opium, subsequent encounter \\
\hline $\mathrm{T} 40.0 \times 5 \mathrm{~S}$ & Adverse effect of opium, sequelae \\
\hline $\mathrm{T} 40.1 \times 1 \mathrm{~A}$ & Poisoning by heroin, accidental (unintentional), initial encounter \\
\hline T40.1X1D & Poisoning by heroin, accidental (unintentional), subsequent encounter \\
\hline T40.1X1S & Poisoning by heroin, accidental (unintentional), sequelae \\
\hline T40.1X4A & Poisoning by heroin, undetermined, initial encounter \\
\hline T40.1X4D & Poisoning by heroin, undetermined, subsequent encounter \\
\hline $\mathrm{T} 40.1 \mathrm{X} 4 \mathrm{~S}$ & Poisoning by heroin, undetermined, sequelae \\
\hline $\mathrm{T} 40.2 \times 1 \mathrm{~A}$ & Poisoning by other opioids, accidental (unintentional), initial encounter \\
\hline T40.2X1D & Poisoning by other opioids, accidental (unintentional), subsequent encounter \\
\hline T40.2X1S & Poisoning by other opioids, accidental (unintentional), sequelae \\
\hline T40.2X4A & Poisoning by other opioids, undetermined, initial encounter \\
\hline T40.2X4D & Poisoning by other opioids, undetermined, subsequent encounter \\
\hline T40.2X4S & Poisoning by other opioids, undetermined, sequelae \\
\hline $\mathrm{T} 40.2 \times 5 \mathrm{~A}$ & Adverse effect of other opioids, initial encounter \\
\hline T40.2X5D & Adverse effect of other opioids, subsequent encounter \\
\hline T40.2X5S & Adverse effect of other opioids, sequelae \\
\hline T40.3X1A & Poisoning by methadone, accidental (unintentional), initial encounter \\
\hline T40.3X1D & Poisoning by methadone, accidental (unintentional), subsequent encounter \\
\hline T40.3X1S & Poisoning by methadone, accidental (unintentional), sequelae \\
\hline T40.3X4A & Poisoning by methadone, undetermined, initial encounter \\
\hline T40.3X4D & Poisoning by methadone, undetermined, subsequent encounter \\
\hline T40.3X4S & Poisoning by methadone, undetermined, sequelae \\
\hline T40.3X5A & Adverse effect of methadone, initial encounter \\
\hline T40.3X5D & Adverse effect of methadone, subsequent encounter \\
\hline T40.3X5S & Adverse effect of methadone, sequelae \\
\hline T40.4X1A & Poisoning by synthetic narcotics, accidental (unintentional), initial encounter \\
\hline T40.4X1D & $\begin{array}{l}\text { Poisoning by synthetic narcotics, accidental (unintentional), subsequent } \\
\text { encounter }\end{array}$ \\
\hline $\mathrm{T} 40.4 \mathrm{X} 1 \mathrm{~S}$ & Poisoning by synthetic narcotics, accidental (unintentional), sequelae \\
\hline $\mathrm{T} 40.4 \mathrm{X} 4 \mathrm{~A}$ & Poisoning by synthetic narcotics, undetermined, initial encounter \\
\hline T40.4X4D & Poisoning by synthetic narcotics, undetermined, subsequent encounter \\
\hline $\mathrm{T} 40.4 \mathrm{X} 4 \mathrm{~S}$ & Poisoning by synthetic narcotics, undetermined, sequelae \\
\hline T40.4X5A & Adverse effect of synthetic narcotics, initial encounter \\
\hline
\end{tabular}




\begin{tabular}{l|l}
\hline T40.4X5D & Adverse effect of synthetic narcotic, subsequent encounter \\
\hline T40.4X5S & Adverse effect of synthetic narcotic, sequelae \\
\hline T40.601A & Poisoning by unspecified narcotics, accidental (unintentional), initial encounter \\
\hline T40.601D & $\begin{array}{l}\text { Poisoning by unspecified narcotics, accidental (unintentional), subsequent } \\
\text { encounter }\end{array}$ \\
\hline T40.601S & Poisoning by unspecified narcotics, accidental (unintentional), sequelae \\
\hline T40.604A & Poisoning by unspecified narcotics, undetermined, initial encounter \\
\hline T40.604D & Poisoning by unspecified narcotics, undetermined, subsequent encounter \\
\hline T40.604S & Poisoning by unspecified narcotics, undetermined, sequelae \\
\hline T40.605A & Adverse effect of unspecified narcotics, initial encounter \\
\hline T40.605D & Adverse effect of unspecified narcotics, subsequent encounter \\
\hline T40.605S & Adverse effect of unspecified narcotics, sequelae \\
\hline T40.691A & Poisoning by other narcotics, accidental (unintentional), initial encounter \\
\hdashline T40.691D & Poisoning by other narcotics, accidental (unintentional), subsequent encounter \\
\hline T40.691S & Poisoning by other narcotics, accidental (unintentional), sequelae \\
\hline T40.694A & Poisoning by other narcotics, undetermined, initial encounter \\
\hline T40.694D & Poisoning by other narcotics, undetermined, subsequent encounter \\
\hline T40.694S & Poisoning by other narcotics, undetermined, sequelae \\
\hline T40.695A & Adverse effect of other narcotics, initial encounter \\
\hline T40.695D & Adverse effect of other narcotics, subsequent encounter \\
\hline T40.695S & Adverse effect of other narcotics, sequelae \\
\hline
\end{tabular}

(ICD10Data.com, 2018) 


\section{Appendix I: VHA Study Cohort Consort Diagram}

139 Unique Facilities

15,420 Unique Index Hospitalizations

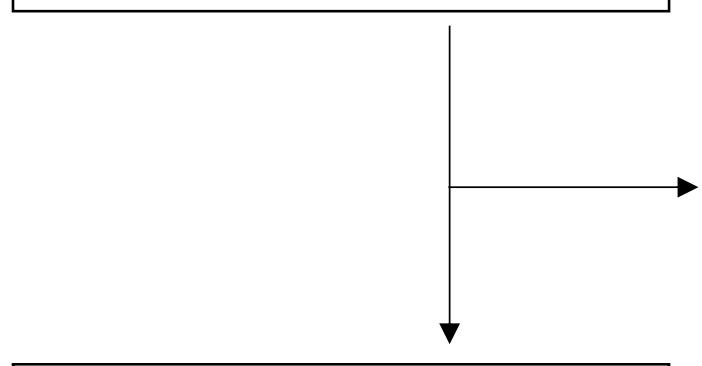

132 Unique Facilities

15,136 Unique Index Hospitalizations

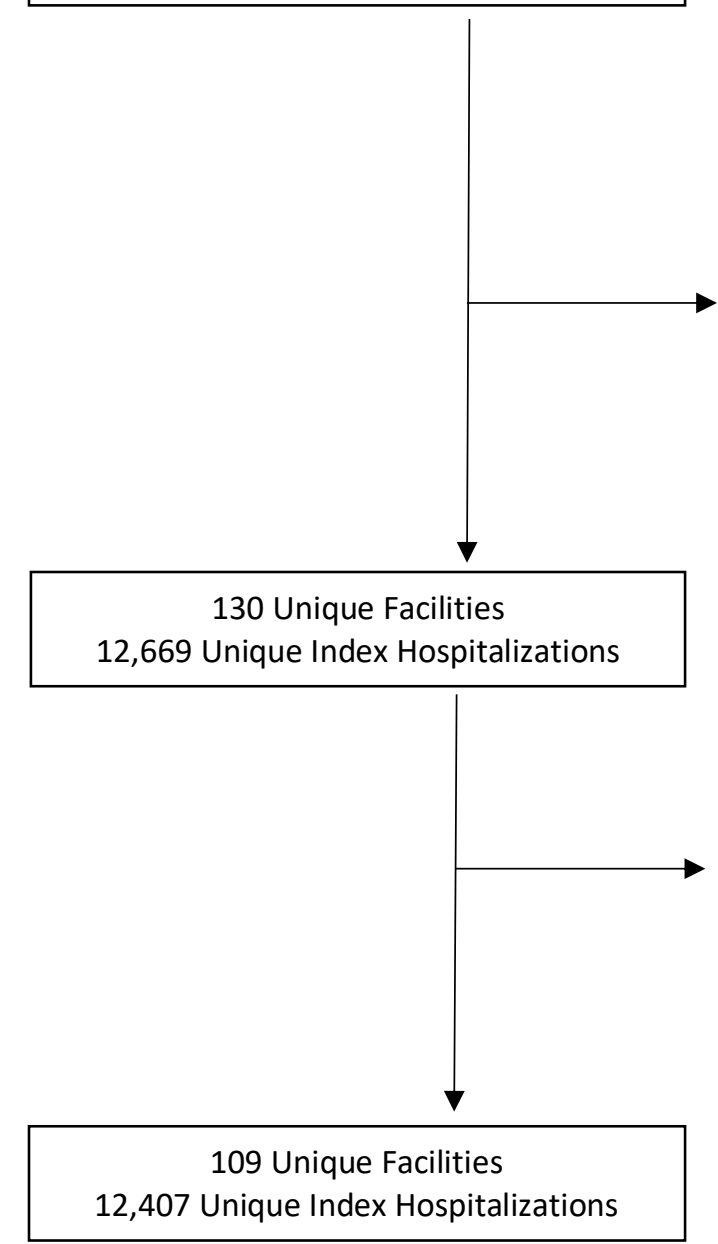

Step 1. Exclude Puerto Rico facility (hospitals $=1$; cases $=134$ ).

Step 2. Exclude facilities with bed category discrepancies (hospitals $=6$; cases $=141$ ).

Step 3. Exclude cases with non-Hospital place admissions (cases $=7$ ) or missing place of admission (cases $=2$ ).

Step 4. Exclude cases missing primary or secondary admission diagnosis $(n=271)$.

Step 5. Exclude cases with inconsistent death variable (cases $=4$ ); lost a facility here (hospitals = 1).

Step 6. Exclude cases with cancer flag (see definition in next section) - solid tumor w/o metastatic, metastatic cancer, and lymphoma (cases $=2062$ ).

Step 7. Exclude cases with a length of stay >= 51 days ( $99^{\text {th }}$ percentile) (cases $\left.=130\right)$; lost a facility here (hospitals $=1$ ).

Step 8. Excluded facilities based on minimum observations $<25$ (hospitals $=21$; cases $=$ 262).

(CONSORT, 2010) 


\section{Appendix J: Patient and Hospital Study Cohort Definitions}

\section{Acronyms}

$\mathrm{CDW}=$ corporate data warehouse; $\mathrm{VHA}=$ Veterans Health Administration.

\section{Index Hospitalization}

Index hospitalization was defined as the first acute hospitalization per patient with an OUD diagnosis as defined below in the VHA 2017 fiscal year (discharge date between 10/1/2016 and 10/1/2017). Index hospitalizations were included in the study cohort for an OUD diagnosis at the time of hospitalization in any of the "Present on Admission" fields or any number of the diagnosis fields for that hospital stay or any OUD diagnosis for any encounter outpatient or inpatient within the 12 months prior to index hospitalization.

\section{OUD Diagnosis}

Patients with an OUD diagnosis, from any source, inpatient or outpatient, between 10/1/2015 and 10/1/2017 in the year preceding the discharge date of index hospitalization. This could include an OUD diagnosis during index hospitalization and the diagnosis could be at any of the 24 diagnoses positions.

\section{Hospitalization fields searched}

1. Present on admission: "Inpat.PresentOnAdmission"

2. Summary diagnoses: "Inpat.InpatientDiagnosis"

3. Discharge diagnoses: "Inpat.InpatientDischargeDiagnosis"

4. Diagnoses associated with patient transfer within the hospital: "Inpat.PatientTransferDiagnosis"

5. Diagnoses associated with specialty transfer within the hospital: "Inpat.SpecialtyTransferDiagnosis"

6. Fee Basis inpatient diagnoses tables

Outpatient fields searched

1. Outpatient diagnosis table

2. Fee Basis Outpatient diagnoses tables

\section{Identification of Cancer Codes: Present on Admission}

Comorbidities were identified in several sources within the CDW (Outpatient, Outpatient FEE BASIS, Inpatient Diagnosis, Inpatient Discharge, Inpatient Transfer Diagnosis, Inpatient Present on Admission, Inpatient Specialty Transfer Diagnosis, Inpatient FEE BASIS). At least one ICD10 diagnosis was present for a patient to receive a flag. Conditions were identified within 365 days prior to the index admission date. The Elixhauser classification system (Agency for Healthcare Research and Quality, 2018) was used: LYMPH = 'Lymphoma'; METS = 'Metastatic cancer'; TUMOR = 'Solid tumor w/out metastasis.'

\section{Hospital Selection}

Included hospitals were restricted to "acute care hospitals" from a report produced internally at the VHA, which only included hospitals with at least 500 "acute" bed days of service during fiscal year 2017. 


\section{Hospital Size}

Hospital size was defined by the number of operating beds from a document downloaded from the VHA Support Service Center reporting portal. Operating beds were staffed and available for admission of patients. The operating bed count did not include unavailable beds. Occupancy rates were determined for each facility based on current approved operating bed levels. Further, the bed type was restricted to "acute" (e.g., internal medicine, surgery, neurology) and did not include counts for nursing beds or hospice beds (e.g., beds that cannot accommodate acute care patients).

\section{Hospital Identifier}

The hospital identifier, the parent facility, was created by using the VHA station number. A variable from the VHA CDW. 


\section{Appendix K: Patient and Admission Definitions}

\section{Acronyms}

$\mathrm{CDW}=$ corporate data warehouse; $\mathrm{VHA}=$ Veterans Health Administration; SUD = substance use disorder; OUD= opioid use disorder.

Age

Calculated continuous variable for age of patient on the index hospitalization admission date.

Values ranged from 21 to 90 . Patients aged $>90$ were listed at 90.

\section{Co-Occurring Mental Health Condition: Present on Admission}

Co-occurring mental health conditions were identified in several sources within the CDW (Outpatient, Outpatient FEE BASIS, Inpatient Diagnosis, Inpatient Discharge, Inpatient Transfer Diagnosis, Inpatient Present on Admission, Inpatient Specialty Transfer Diagnosis, Inpatient FEE BASIS). At least one ICD-10 diagnosis must have been present for a patient to receive a positive flag. See Appendix L for ICD-10 code list. Conditions were identified within 365 days prior to the index hospitalization admission date: Adjustment disorder other; anxiety disorder; mood disorder; non-mood psychotic disorder; post-traumatic stress disorder; and self-harm.

\section{Co-Occurring SUD: Present on Admission}

Co-occurring SUDs were identified in several sources within the CDW (Outpatient, Outpatient FEE BASIS, Inpatient Diagnosis, Inpatient Discharge, Inpatient Transfer Diagnosis, Inpatient Present on Admission, Inpatient Specialty Transfer Diagnosis, Inpatient FEE BASIS). At least one ICD-10 diagnosis must have been present for a patient to receive a positive flag. See Appendix $L$ for ICD-10 code list. Conditions were identified within 365 days prior to the index hospitalization admission date: Alcohol use disorder; cannabis use disorder; cocaine use disorder; hallucinogen use disorder; nicotine dependence; other psychoactive use disorders; other stimulant related disorders; other substance use disorder; and sedative hypnotic disorder.

\section{Gender}

VHA variable for gender of the patient: male or female.

\section{Ethnicity}

VHA variable describing the patient's ethnicity: Not Hispanic or Latino; Hispanic Or Latino; and Unknown.

\section{OUD-Related Infection: During Admission}

OUD-related infections during index hospitalization were captured using the following tables from the CDW: Inpatient Diagnosis, Inpatient Discharge, Inpatient Transfer Diagnosis, Inpatient Present on Admission, Inpatient Specialty Transfer Diagnosis. The presence of a least one ICD-10 diagnosis in one of those tables must have been present during admission for a patient to receive a positive flag. See Appendix M for ICD-10 code list: Endocarditis; candida endocarditis; osteomyelitis; bacteremia; discitis; septic arthritis; brain abscess; joint infection; necrotizing fasciitis; empyema; and lung abscess. 


\section{OUD-Related Diagnosis: During Admission}

An OUD-related diagnosis during admission occurred in either the primary or secondary diagnosis code location for the index hospitalization from the "Inpat.InpatientDiagnosis" table. The ICD-10 codes were transformed using the ICD Rstudio package (Wasey, 2018).

- All OUD Codes: Primary: A variable that indicates whether an F or T code for OUD was in the primary diagnosis position. Included codes are listed in Appendix $\mathrm{H}$.

- All OUD Codes: Secondary: A variable that indicates whether an F or T code for OUD was in the secondary. Included codes are listed in Appendix $\mathrm{H}$.

- Any OUD Code: A primary or secondary OUD code. Included codes are listed in Appendix $\mathrm{H}$.

\section{Primary or Secondary Diagnosis Code: During Admission}

The primary or secondary diagnosis code captured the primary or secondary diagnosis codes for the index hospitalization from the "Inpat.InpatientDiagnosis" table. The ICD-10 codes were transformed using the ICD RStudio package (Wasey, 2018).

\section{Race}

The race variable used in this study was the "Race 1" category. The original 8 categories were collapsed to 6 categories: Unknown; Native American; Black; Asian; Native Islander; and White.

\section{Admission Source}

The admission source variable indicated the source for the admission. These entries come from VISTA, and each local health care system devises their own coding scheme and abbreviations. The original 18 categories were collapsed into 3 categories: outpatient treatment; other direct admission; and other.

\section{ICU Services Received}

The ICU variable indicated whether there was an ICU bed stay (i.e., medical, surgical, cardiac, neurosurgery) associated with the index hospitalization.

\section{Surgical Services Received}

The surgical services variable indicated whether there was a surgery bed stay (i.e., general surgery, cardiac surgery, surgical step-down, thoracic surgery, oral surgery, plastic surgery) associated with the index hospitalization.

\section{Length of Stay}

The length of stay variable was calculated based on the number of days of index hospitalization. If admitted/discharged on the same day this was represented as 1 day. The values ranged from 1 to 1652 , and $90 \%$ of values were between 1 and 15 days. 


\section{Appendix L: Co-Occurring Mental Health and Substance Use Disorder Diagnosis Codes Queried}

\begin{tabular}{|c|c|}
\hline $\begin{array}{c}\text { ICD10 } \\
\text { Code }\end{array}$ & ICD10 Long Description \\
\hline F4321 & Adjustment disorder with depressed mood \\
\hline F4322 & Adjustment disorder with anxiety \\
\hline F4323 & Adjustment disorder with mixed anxiety and depressed mood \\
\hline F4324 & Adjustment disorder with disturbance of conduct \\
\hline F4325 & Adjustment disorder with mixed disturbance of emotions and conduct \\
\hline F4329 & Adjustment disorder with other symptoms \\
\hline F438 & Other reactions to severe stress \\
\hline F439 & Reaction to severe stress, unspecified \\
\hline F4320 & Adjustment disorders, unspecified \\
\hline F1010 & Alcohol abuse, uncomplicated \\
\hline F10120 & Alcohol abuse with intoxication, uncomplicated \\
\hline F10121 & Alcohol abuse with intoxication delirium \\
\hline F10129 & Alcohol abuse with intoxication, unspecified \\
\hline F1014 & Alcohol abuse with alcohol-induced mood disorder \\
\hline F10150 & Alcohol abuse with alcohol-induced psychotic disorder with delusions \\
\hline F10151 & Alcohol abuse with alcohol-induced psychotic disorder with hallucinations \\
\hline F10159 & Alcohol abuse with alcohol-induced psychotic disorder, unspecified \\
\hline F10180 & Alcohol abuse with alcohol-induced anxiety disorder \\
\hline F10181 & Alcohol abuse with alcohol-induced sexual dysfunction \\
\hline F10182 & Alcohol abuse with alcohol-induced sleep disorder \\
\hline F10188 & Alcohol abuse with other alcohol-induced disorder \\
\hline F1019 & Alcohol abuse with unspecified alcohol-induced disorder \\
\hline F1020 & Alcohol dependence, uncomplicated \\
\hline F1021 & Alcohol dependence, in remission \\
\hline F10220 & Alcohol dependence with intoxication, uncomplicated \\
\hline F10221 & Alcohol dependence with intoxication delirium \\
\hline F10229 & Alcohol dependence with intoxication, unspecified \\
\hline F10230 & Alcohol dependence with withdrawal, uncomplicated \\
\hline F10231 & Alcohol dependence with withdrawal delirium \\
\hline F10232 & Alcohol dependence with withdrawal with perceptual disturbance \\
\hline F10239 & Alcohol dependence with withdrawal, unspecified \\
\hline F1024 & Alcohol dependence with alcohol-induced mood disorder \\
\hline F10250 & Alcohol dependence with alcohol-induced psychotic disorder with delusions \\
\hline F10251 & Alcohol dependence with alcohol-induced psychotic disorder with hallucinations \\
\hline
\end{tabular}




\begin{tabular}{|c|c|}
\hline F10259 & Alcohol dependence with alcohol-induced psychotic disorder, unspecified \\
\hline F1026 & Alcohol dependence with alcohol-induced persisting amnestic disorder \\
\hline F1027 & Alcohol dependence with alcohol-induced persisting dementia \\
\hline F10280 & Alcohol dependence with alcohol-induced anxiety disorder \\
\hline F10281 & Alcohol dependence with alcohol-induced sexual dysfunction \\
\hline F10282 & Alcohol dependence with alcohol-induced sleep disorder \\
\hline F10288 & Alcohol dependence with other alcohol-induced disorder \\
\hline F1029 & Alcohol dependence with unspecified alcohol-induced disorder \\
\hline F10920 & Alcohol use, unspecified with intoxication, uncomplicated \\
\hline F10921 & Alcohol use, unspecified with intoxication delirium \\
\hline F10929 & Alcohol use, unspecified with intoxication, unspecified \\
\hline F1094 & Alcohol use, unspecified with alcohol-induced mood disorder \\
\hline F10950 & Alcohol use, unspecified with alcohol-induced psychotic disorder with delusions \\
\hline F10951 & Alcohol use, unspecified with alcohol-induced psychotic disorder with hallucinations \\
\hline F10959 & Alcohol use, unspecified with alcohol-induced psychotic disorder, unspecified \\
\hline F1096 & Alcohol use, unspecified with alcohol-induced persisting amnestic disorder \\
\hline F1097 & Alcohol use, unspecified with alcohol-induced persisting dementia \\
\hline F10980 & Alcohol use, unspecified with alcohol-induced anxiety disorder \\
\hline F10981 & Alcohol use, unspecified with alcohol-induced sexual dysfunction \\
\hline F10982 & Alcohol use, unspecified with alcohol-induced sleep disorder \\
\hline F10988 & Alcohol use, unspecified with other alcohol-induced disorder \\
\hline F1099 & Alcohol use, unspecified with unspecified alcohol-induced disorder \\
\hline F4000 & Agoraphobia, unspecified \\
\hline F4001 & Agoraphobia with panic disorder \\
\hline F4002 & Agoraphobia without panic disorder \\
\hline F4010 & Social phobia, unspecified \\
\hline F4011 & Social phobia, generalized \\
\hline F40210 & Arachnophobia \\
\hline F40218 & Other animal type phobia \\
\hline F40220 & Fear of thunderstorms \\
\hline F40228 & Other natural environment type phobia \\
\hline F40230 & Fear of blood \\
\hline F40231 & Fear of injections and transfusions \\
\hline F40232 & Fear of other medical care \\
\hline F40233 & Fear of injury \\
\hline F40240 & Claustrophobia \\
\hline F40241 & Acrophobia \\
\hline F40242 & Fear of bridges \\
\hline F40243 & Fear of flying \\
\hline
\end{tabular}




\begin{tabular}{|c|c|}
\hline F40248 & Other situational type phobia \\
\hline F40290 & Androphobia \\
\hline F40291 & Gynephobia \\
\hline F40298 & Other specified phobia \\
\hline F408 & Other phobic anxiety disorders \\
\hline F409 & Phobic anxiety disorder, unspecified \\
\hline F410 & Panic disorder [episodic paroxysmal anxiety] without agoraphobia \\
\hline F411 & Generalized anxiety disorder \\
\hline F413 & Other mixed anxiety disorders \\
\hline F418 & Other specified anxiety disorders \\
\hline F419 & Anxiety disorder, unspecified \\
\hline F42 & Obsessive-compulsive disorder \\
\hline F1210 & Cannabis abuse, uncomplicated \\
\hline F12120 & Cannabis abuse with intoxication, uncomplicated \\
\hline F12121 & Cannabis abuse with intoxication delirium \\
\hline F12122 & Cannabis abuse with intoxication with perceptual disturbance \\
\hline F12129 & Cannabis abuse with intoxication, unspecified \\
\hline F12150 & Cannabis abuse with psychotic disorder with delusions \\
\hline F12151 & Cannabis abuse with psychotic disorder with hallucinations \\
\hline F12159 & Cannabis abuse with psychotic disorder, unspecified \\
\hline F12180 & Cannabis abuse with cannabis-induced anxiety disorder \\
\hline F12188 & Cannabis abuse with other cannabis-induced disorder \\
\hline F1219 & Cannabis abuse with unspecified cannabis-induced disorder \\
\hline F1220 & Cannabis dependence, uncomplicated \\
\hline F1221 & Cannabis dependence, in remission \\
\hline F12220 & Cannabis dependence with intoxication, uncomplicated \\
\hline F12221 & Cannabis dependence with intoxication delirium \\
\hline F12222 & Cannabis dependence with intoxication with perceptual disturbance \\
\hline F12229 & Cannabis dependence with intoxication, unspecified \\
\hline F12250 & Cannabis dependence with psychotic disorder with delusions \\
\hline F12251 & Cannabis dependence with psychotic disorder with hallucinations \\
\hline F12259 & Cannabis dependence with psychotic disorder, unspecified \\
\hline F12280 & Cannabis dependence with cannabis-induced anxiety disorder \\
\hline F12288 & Cannabis dependence with other cannabis-induced disorder \\
\hline F1229 & Cannabis dependence with unspecified cannabis-induced disorder \\
\hline F1290 & Cannabis use, unspecified, uncomplicated \\
\hline F12920 & Cannabis use, unspecified with intoxication, uncomplicated \\
\hline F12921 & Cannabis use, unspecified with intoxication delirium \\
\hline F12922 & Cannabis use, unspecified with intoxication with perceptual disturbance \\
\hline
\end{tabular}




\begin{tabular}{|c|c|}
\hline F12929 & Cannabis use, unspecified with intoxication, unspecified \\
\hline F12950 & Cannabis use, unspecified with psychotic disorder with delusions \\
\hline F12951 & Cannabis use, unspecified with psychotic disorder with hallucinations \\
\hline F12959 & Cannabis use, unspecified with psychotic disorder, unspecified \\
\hline F12980 & Cannabis use, unspecified with anxiety disorder \\
\hline F12988 & Cannabis use, unspecified with other cannabis-induced disorder \\
\hline F1299 & Cannabis use, unspecified with unspecified cannabis-induced disorder \\
\hline F14121 & Cocaine abuse with intoxication with delirium \\
\hline F14122 & Cocaine abuse with intoxication with perceptual disturbance \\
\hline F14129 & Cocaine abuse with intoxication, unspecified \\
\hline F1414 & Cocaine abuse with cocaine-induced mood disorder \\
\hline F14150 & Cocaine abuse with cocaine-induced psychotic disorder with delusions \\
\hline F14151 & Cocaine abuse with cocaine-induced psychotic disorder with hallucinations \\
\hline F14159 & Cocaine abuse with cocaine-induced psychotic disorder, unspecified \\
\hline F14180 & Cocaine abuse with cocaine-induced anxiety disorder \\
\hline F14181 & Cocaine abuse with cocaine-induced sexual dysfunction \\
\hline F14182 & Cocaine abuse with cocaine-induced sleep disorder \\
\hline F14188 & Cocaine abuse with other cocaine-induced disorder \\
\hline F1419 & Cocaine abuse with unspecified cocaine-induced disorder \\
\hline F14220 & Cocaine dependence with intoxication, uncomplicated \\
\hline F14221 & Cocaine dependence with intoxication delirium \\
\hline F14222 & Cocaine dependence with intoxication with perceptual disturbance \\
\hline F14229 & Cocaine dependence with intoxication, unspecified \\
\hline F1423 & Cocaine dependence with withdrawal \\
\hline F1424 & Cocaine dependence with cocaine-induced mood disorder \\
\hline F14250 & Cocaine dependence with cocaine-induced psychotic disorder with delusions \\
\hline F14251 & Cocaine dependence with cocaine-induced psychotic disorder with hallucinations \\
\hline F14259 & Cocaine dependence with cocaine-induced psychotic disorder, unspecified \\
\hline F14280 & Cocaine dependence with cocaine-induced anxiety disorder \\
\hline F14281 & Cocaine dependence with cocaine-induced sexual dysfunction \\
\hline F14282 & Cocaine dependence with cocaine-induced sleep disorder \\
\hline F14288 & Cocaine dependence with other cocaine-induced disorder \\
\hline F1429 & Cocaine dependence with unspecified cocaine-induced disorder \\
\hline F14920 & Cocaine use, unspecified with intoxication, uncomplicated \\
\hline F14921 & Cocaine use, unspecified with intoxication delirium \\
\hline F14922 & Cocaine use, unspecified with intoxication with perceptual disturbance \\
\hline F14929 & Cocaine use, unspecified with intoxication, unspecified \\
\hline F1494 & Cocaine use, unspecified with cocaine-induced mood disorder \\
\hline F14950 & Cocaine use, unspecified with cocaine-induced psychotic disorder with delusions \\
\hline
\end{tabular}




\begin{tabular}{|c|c|}
\hline F14951 & Cocaine use, unspecified with cocaine-induced psychotic disorder with hallucinations \\
\hline F14959 & Cocaine use, unspecified with cocaine-induced psychotic disorder, unspecified \\
\hline F14980 & Cocaine use, unspecified with cocaine-induced anxiety disorder \\
\hline F14981 & Cocaine use, unspecified with cocaine-induced sexual dysfunction \\
\hline F14982 & Cocaine use, unspecified with cocaine-induced sleep disorder \\
\hline F14988 & Cocaine use, unspecified with other cocaine-induced disorder \\
\hline F1499 & Cocaine use, unspecified with unspecified cocaine-induced disorder \\
\hline F1610 & Hallucinogen abuse, uncomplicated \\
\hline F16120 & Hallucinogen abuse with intoxication, uncomplicated \\
\hline F16121 & Hallucinogen abuse with intoxication with delirium \\
\hline F16122 & Hallucinogen abuse with intoxication with perceptual disturbance \\
\hline F16129 & Hallucinogen abuse with intoxication, unspecified \\
\hline F1614 & Hallucinogen abuse with hallucinogen-induced mood disorder \\
\hline F16150 & Hallucinogen abuse with hallucinogen-induced psychotic disorder with delusions \\
\hline F16151 & Hallucinogen abuse with hallucinogen-induced psychotic disorder with hallucinations \\
\hline F16159 & Hallucinogen abuse with hallucinogen-induced psychotic disorder, unspecified \\
\hline F16180 & Hallucinogen abuse with hallucinogen-induced anxiety disorder \\
\hline F16183 & Hallucinogen abuse with hallucinogen persisting perception disorder (flashbacks) \\
\hline F16188 & Hallucinogen abuse with other hallucinogen-induced disorder \\
\hline F1619 & Hallucinogen abuse with unspecified hallucinogen-induced disorder \\
\hline F1620 & Hallucinogen dependence, uncomplicated \\
\hline F1621 & Hallucinogen dependence, in remission \\
\hline F16220 & Hallucinogen dependence with intoxication, uncomplicated \\
\hline F16221 & Hallucinogen dependence with intoxication with delirium \\
\hline F16229 & Hallucinogen dependence with intoxication, unspecified \\
\hline F1624 & Hallucinogen dependence with hallucinogen-induced mood disorder \\
\hline F16250 & Hallucinogen dependence with hallucinogen-induced psychotic disorder with delusions \\
\hline F16251 & Hallucinogen dependence with hallucinogen-induced psychotic disorder with hallucinations \\
\hline F16259 & Hallucinogen dependence with hallucinogen-induced psychotic disorder, unspecified \\
\hline F16280 & Hallucinogen dependence with hallucinogen-induced anxiety disorder \\
\hline F16283 & Hallucinogen dependence with hallucinogen persisting perception disorder (flashbacks) \\
\hline F16288 & Hallucinogen dependence with other hallucinogen-induced disorder \\
\hline F1629 & Hallucinogen dependence with unspecified hallucinogen-induced disorder \\
\hline F1690 & Hallucinogen use, unspecified, uncomplicated \\
\hline F16920 & Hallucinogen use, unspecified with intoxication, uncomplicated \\
\hline F16921 & Hallucinogen use, unspecified with intoxication with delirium \\
\hline F16929 & Hallucinogen use, unspecified with intoxication, unspecified \\
\hline F1694 & Hallucinogen use, unspecified with hallucinogen-induced mood disorder \\
\hline F16950 & Hallucinogen use, unspecified with hallucinogen-induced psychotic disorder with delusions \\
\hline
\end{tabular}




\begin{tabular}{|c|c|}
\hline F16951 & $\begin{array}{l}\text { Hallucinogen use, unspecified with hallucinogen-induced psychotic disorder with } \\
\text { hallucinations }\end{array}$ \\
\hline F16959 & Hallucinogen use, unspecified with hallucinogen-induced psychotic disorder, unspecified \\
\hline F16980 & Hallucinogen use, unspecified with hallucinogen-induced anxiety disorder \\
\hline F16983 & Hallucinogen use, unspecified with hallucinogen persisting perception disorder (flashbacks) \\
\hline F16988 & Hallucinogen use, unspecified with other hallucinogen-induced disorder \\
\hline F1699 & Hallucinogen use, unspecified with unspecified hallucinogen-induced disorder \\
\hline F18121 & Inhalant abuse with intoxication delirium \\
\hline F18129 & Inhalant abuse with intoxication, unspecified \\
\hline F1814 & Inhalant abuse with inhalant-induced mood disorder \\
\hline F18150 & Inhalant abuse with inhalant-induced psychotic disorder with delusions \\
\hline F18151 & Inhalant abuse with inhalant-induced psychotic disorder with hallucinations \\
\hline F18159 & Inhalant abuse with inhalant-induced psychotic disorder, unspecified \\
\hline F1817 & Inhalant abuse with inhalant-induced dementia \\
\hline F18180 & Inhalant abuse with inhalant-induced anxiety disorder \\
\hline F18188 & Inhalant abuse with other inhalant-induced disorder \\
\hline F1819 & Inhalant abuse with unspecified inhalant-induced disorder \\
\hline F1824 & Inhalant dependence with inhalant-induced mood disorder \\
\hline F18250 & Inhalant dependence with inhalant-induced psychotic disorder with delusions \\
\hline F18251 & Inhalant dependence with inhalant-induced psychotic disorder with hallucinations \\
\hline F18259 & Inhalant dependence with inhalant-induced psychotic disorder, unspecified \\
\hline F1827 & Inhalant dependence with inhalant-induced dementia \\
\hline F18280 & Inhalant dependence with inhalant-induced anxiety disorder \\
\hline F18288 & Inhalant dependence with other inhalant-induced disorder \\
\hline F1829 & Inhalant dependence with unspecified inhalant-induced disorder \\
\hline F18920 & Inhalant use, unspecified with intoxication, uncomplicated \\
\hline F18921 & Inhalant use, unspecified with intoxication with delirium \\
\hline F18929 & Inhalant use, unspecified with intoxication, unspecified \\
\hline F1894 & Inhalant use, unspecified with inhalant-induced mood disorder \\
\hline F18950 & Inhalant use, unspecified with inhalant-induced psychotic disorder with delusions \\
\hline F18951 & Inhalant use, unspecified with inhalant-induced psychotic disorder with hallucinations \\
\hline F18959 & Inhalant use, unspecified with inhalant-induced psychotic disorder, unspecified \\
\hline F1897 & Inhalant use, unspecified with inhalant-induced persisting dementia \\
\hline F18980 & Inhalant use, unspecified with inhalant-induced anxiety disorder \\
\hline F18988 & Inhalant use, unspecified with other inhalant-induced disorder \\
\hline F1899 & Inhalant use, unspecified with unspecified inhalant-induced disorder \\
\hline F1810 & Inhalant abuse, uncomplicated \\
\hline F18120 & Inhalant abuse with intoxication, uncomplicated \\
\hline F1820 & Inhalant dependence, uncomplicated \\
\hline F1821 & Inhalant dependence, in remission \\
\hline
\end{tabular}




\begin{tabular}{|c|c|}
\hline F18220 & Inhalant dependence with intoxication, uncomplicated \\
\hline F18221 & Inhalant dependence with intoxication delirium \\
\hline F18229 & Inhalant dependence with intoxication, unspecified \\
\hline F1890 & Inhalant use, unspecified, uncomplicated \\
\hline F3010 & Manic episode without psychotic symptoms, unspecified \\
\hline F3011 & Manic episode without psychotic symptoms, mild \\
\hline F3012 & Manic episode without psychotic symptoms, moderate \\
\hline F3013 & Manic episode, severe, without psychotic symptoms \\
\hline F302 & Manic episode, severe with psychotic symptoms \\
\hline F303 & Manic episode in partial remission \\
\hline F304 & Manic episode in full remission \\
\hline F308 & Other manic episodes \\
\hline F309 & Manic episode, unspecified \\
\hline F310 & Bipolar disorder, current episode hypomanic \\
\hline F3110 & Bipolar disorder, current episode manic without psychotic features, unspecified \\
\hline F3111 & Bipolar disorder, current episode manic without psychotic features, mild \\
\hline F3112 & Bipolar disorder, current episode manic without psychotic features, moderate \\
\hline F3113 & Bipolar disorder, current episode manic without psychotic features, severe \\
\hline F312 & Bipolar disorder, current episode manic severe with psychotic features \\
\hline F3130 & Bipolar disorder, current episode depressed, mild or moderate severity, unspecified \\
\hline F3131 & Bipolar disorder, current episode depressed, mild \\
\hline F3132 & Bipolar disorder, current episode depressed, moderate \\
\hline F314 & Bipolar disorder, current episode depressed, severe, without psychotic features \\
\hline F315 & Bipolar disorder, current episode depressed, severe, with psychotic features \\
\hline F3160 & Bipolar disorder, current episode mixed, unspecified \\
\hline F3161 & Bipolar disorder, current episode mixed, mild \\
\hline F3162 & Bipolar disorder, current episode mixed, moderate \\
\hline F3163 & Bipolar disorder, current episode mixed, severe, without psychotic features \\
\hline F3164 & Bipolar disorder, current episode mixed, severe, with psychotic features \\
\hline F3170 & Bipolar disorder, currently in remission, most recent episode unspecified \\
\hline F3171 & Bipolar disorder, in partial remission, most recent episode hypomanic \\
\hline F3172 & Bipolar disorder, in full remission, most recent episode hypomanic \\
\hline F3173 & Bipolar disorder, in partial remission, most recent episode manic \\
\hline F3174 & Bipolar disorder, in full remission, most recent episode manic \\
\hline F3175 & Bipolar disorder, in partial remission, most recent episode depressed \\
\hline F3176 & Bipolar disorder, in full remission, most recent episode depressed \\
\hline F3177 & Bipolar disorder, in partial remission, most recent episode mixed \\
\hline F3178 & Bipolar disorder, in full remission, most recent episode mixed \\
\hline F3181 & Bipolar II disorder \\
\hline
\end{tabular}




\begin{tabular}{|c|c|}
\hline F3189 & Other bipolar disorder \\
\hline F319 & Bipolar disorder, unspecified \\
\hline F320 & Major depressive disorder, single episode, mild \\
\hline F321 & Major depressive disorder, single episode, moderate \\
\hline F322 & Major depressive disorder, single episode, severe without psychotic features \\
\hline F323 & Major depressive disorder, single episode, severe with psychotic features \\
\hline F324 & Major depressive disorder, single episode, in partial remission \\
\hline F325 & Major depressive disorder, single episode, in full remission \\
\hline F328 & Other depressive episodes \\
\hline F329 & Major depressive disorder, single episode, unspecified \\
\hline F330 & Major depressive disorder, recurrent, mild \\
\hline F331 & Major depressive disorder, recurrent, moderate \\
\hline F332 & Major depressive disorder, recurrent severe without psychotic features \\
\hline F333 & Major depressive disorder, recurrent, severe with psychotic symptoms \\
\hline F3340 & Major depressive disorder, recurrent, in remission, unspecified \\
\hline F3341 & Major depressive disorder, recurrent, in partial remission \\
\hline F3342 & Major depressive disorder, recurrent, in full remission \\
\hline F339 & Major depressive disorder, recurrent, unspecified \\
\hline F341 & Dysthymic disorder \\
\hline F390 & Unspecified mood [affective] disorder \\
\hline F17203 & Nicotine dependence unspecified, with withdrawal \\
\hline F17208 & Nicotine dependence, unspecified, with other nicotine-induced disorders \\
\hline F17209 & Nicotine dependence, unspecified, with unspecified nicotine-induced disorders \\
\hline F17213 & Nicotine dependence, cigarettes, with withdrawal \\
\hline F17218 & Nicotine dependence, cigarettes, with other nicotine-induced disorders \\
\hline F17219 & Nicotine dependence, cigarettes, with unspecified nicotine-induced disorders \\
\hline F17223 & Nicotine dependence, chewing tobacco, with withdrawal \\
\hline F17228 & Nicotine dependence, chewing tobacco, with other nicotine-induced disorders \\
\hline F17229 & Nicotine dependence, chewing tobacco, with unspecified nicotine-induced disorders \\
\hline F17293 & Nicotine dependence, other tobacco product, with withdrawal \\
\hline F17298 & Nicotine dependence, other tobacco product, with other nicotine-induced disorders \\
\hline F17299 & Nicotine dependence, other tobacco product, with unspecified nicotine-induced disorders \\
\hline F200 & Paranoid schizophrenia \\
\hline F201 & Disorganized schizophrenia \\
\hline F202 & Catatonic schizophrenia \\
\hline F203 & Undifferentiated schizophrenia \\
\hline F205 & Residual schizophrenia \\
\hline F2081 & Schizophreniform disorder \\
\hline F2089 & Other schizophrenia \\
\hline
\end{tabular}




\begin{tabular}{|c|c|}
\hline F209 & Schizophrenia, unspecified \\
\hline F22 & Delusional disorders \\
\hline F23 & Brief psychotic disorder \\
\hline F24 & Shared psychotic disorder \\
\hline $\mathrm{F} 250$ & Schizoaffective disorder, bipolar type \\
\hline F251 & Schizoaffective disorder, depressive type \\
\hline F258 & Other schizoaffective disorders \\
\hline F259 & Schizoaffective disorder, unspecified \\
\hline F28 & Other psychotic disorder not due to a substance or known physiological condition \\
\hline F29 & Unspecified psychosis not due to a substance or known physiological condition \\
\hline F4489 & Other dissociative and conversion disorders \\
\hline F1910 & Other psychoactive substance abuse, uncomplicated \\
\hline F19120 & Other psychoactive substance abuse with intoxication, uncomplicated \\
\hline F19121 & Other psychoactive substance abuse with intoxication delirium \\
\hline F19122 & Other psychoactive substance abuse with intoxication with perceptual disturbances \\
\hline F19129 & Other psychoactive substance abuse with intoxication, unspecified \\
\hline F1914 & Other psychoactive substance abuse with psychoactive substance-induced mood disorder \\
\hline F19150 & $\begin{array}{l}\text { Other psychoactive substance abuse with psychoactive substance-induced psychotic } \\
\text { disorder with delusions }\end{array}$ \\
\hline F19151 & $\begin{array}{l}\text { Other psychoactive substance abuse with psychoactive substance-induced psychotic } \\
\text { disorder with hallucinations }\end{array}$ \\
\hline F19159 & $\begin{array}{l}\text { Other psychoactive substance abuse with psychoactive substance-induced psychotic } \\
\text { disorder, unspecified }\end{array}$ \\
\hline F1916 & $\begin{array}{l}\text { Other psychoactive substance abuse with psychoactive substance-induced persisting } \\
\text { amnestic disorder }\end{array}$ \\
\hline F1917 & $\begin{array}{l}\text { Other psychoactive substance abuse with psychoactive substance-induced persisting } \\
\text { dementia }\end{array}$ \\
\hline F19180 & Other psychoactive substance abuse with psychoactive substance-induced anxiety disorder \\
\hline F19181 & $\begin{array}{l}\text { Other psychoactive substance abuse with psychoactive substance-induced sexual } \\
\text { dysfunction }\end{array}$ \\
\hline F19182 & Other psychoactive substance abuse with psychoactive substance-induced sleep disorder \\
\hline F19188 & Other psychoactive substance abuse with other psychoactive substance-induced disorder \\
\hline F1919 & $\begin{array}{l}\text { Other psychoactive substance abuse with unspecified psychoactive substance-induced } \\
\text { disorder }\end{array}$ \\
\hline F1920 & Other psychoactive substance dependence, uncomplicated \\
\hline F1921 & Other psychoactive substance dependence, in remission \\
\hline F19220 & Other psychoactive substance dependence with intoxication, uncomplicated \\
\hline F19221 & Other psychoactive substance dependence with intoxication delirium \\
\hline F19222 & Other psychoactive substance dependence with intoxication with perceptual disturbance \\
\hline F19229 & Other psychoactive substance dependence with intoxication, unspecified \\
\hline F19230 & Other psychoactive substance dependence with withdrawal, uncomplicated \\
\hline F19231 & Other psychoactive substance dependence with withdrawal delirium \\
\hline F19232 & Other psychoactive substance dependence with withdrawal with perceptual disturbance \\
\hline
\end{tabular}




\begin{tabular}{|c|c|}
\hline F19239 & Other psychoactive substance dependence with withdrawal, unspecified \\
\hline F1924 & $\begin{array}{l}\text { Other psychoactive substance dependence with psychoactive substance-induced mood } \\
\text { disorder }\end{array}$ \\
\hline F19250 & $\begin{array}{l}\text { Other psychoactive substance dependence with psychoactive substance-induced psychotic } \\
\text { disorder with delusions }\end{array}$ \\
\hline F19251 & $\begin{array}{l}\text { Other psychoactive substance dependence with psychoactive substance-induced psychotic } \\
\text { disorder with hallucinations }\end{array}$ \\
\hline F19259 & $\begin{array}{l}\text { Other psychoactive substance dependence with psychoactive substance-induced psychotic } \\
\text { disorder, unspecified }\end{array}$ \\
\hline F1926 & $\begin{array}{l}\text { Other psychoactive substance dependence with psychoactive substance-induced persisting } \\
\text { amnestic disorder }\end{array}$ \\
\hline F1927 & $\begin{array}{l}\text { Other psychoactive substance dependence with psychoactive substance-induced persisting } \\
\text { dementia }\end{array}$ \\
\hline F19280 & $\begin{array}{l}\text { Other psychoactive substance dependence with psychoactive substance-induced anxiety } \\
\text { disorder }\end{array}$ \\
\hline F19281 & $\begin{array}{l}\text { Other psychoactive substance dependence with psychoactive substance-induced sexual } \\
\text { dysfunction }\end{array}$ \\
\hline F19282 & $\begin{array}{l}\text { Other psychoactive substance dependence with psychoactive substance-induced sleep } \\
\text { disorder }\end{array}$ \\
\hline F19288 & $\begin{array}{l}\text { Other psychoactive substance dependence with other psychoactive substance-induced } \\
\text { disorder }\end{array}$ \\
\hline F1929 & $\begin{array}{l}\text { Other psychoactive substance dependence with unspecified psychoactive substance- } \\
\text { induced disorder }\end{array}$ \\
\hline F1990 & Other psychoactive substance use, unspecified, uncomplicated \\
\hline F19920 & Other psychoactive substance use, unspecified with intoxication, uncomplicated \\
\hline F19921 & Other psychoactive substance use, unspecified with intoxication with delirium \\
\hline F19922 & $\begin{array}{l}\text { Other psychoactive substance use, unspecified with intoxication with perceptual } \\
\text { disturbance }\end{array}$ \\
\hline F19929 & Other psychoactive substance use, unspecified with intoxication, unspecified \\
\hline F19930 & Other psychoactive substance use, unspecified with withdrawal, uncomplicated \\
\hline F19931 & Other psychoactive substance use, unspecified with withdrawal delirium \\
\hline F19932 & Other psychoactive substance use, unspecified with withdrawal with perce \\
\hline F19939 & Other psychoactive substance use, unspecified with withdrawal, unspecified \\
\hline F1994 & $\begin{array}{l}\text { Other psychoactive substance use, unspecified with psychoactive substance-induced mood } \\
\text { disorder }\end{array}$ \\
\hline F19950 & $\begin{array}{l}\text { Other psychoactive substance use, unspecified with psychoactive substance-induced } \\
\text { psychotic disorder with delusions }\end{array}$ \\
\hline F19951 & $\begin{array}{l}\text { Other psychoactive substance use, unspecified with psychoactive substance-induced } \\
\text { psychotic disorder with hallucinations }\end{array}$ \\
\hline F19959 & $\begin{array}{l}\text { Other psychoactive substance use, unspecified with psychoactive substance-induced } \\
\text { psychotic disorder, unspecified }\end{array}$ \\
\hline F1996 & $\begin{array}{l}\text { Other psychoactive substance use, unspecified with psychoactive substance-induced } \\
\text { persisting amnestic disorder }\end{array}$ \\
\hline F1997 & $\begin{array}{l}\text { Other psychoactive substance use, unspecified with psychoactive substance-induced } \\
\text { persisting dementia }\end{array}$ \\
\hline F19980 & $\begin{array}{l}\text { Other psychoactive substance use, unspecified with psychoactive substance-induced anxiety } \\
\text { disorder }\end{array}$ \\
\hline F19981 & $\begin{array}{l}\text { Other psychoactive substance use, unspecified with psychoactive substance-induced sexual } \\
\text { dysfunction }\end{array}$ \\
\hline
\end{tabular}




\begin{tabular}{|c|c|}
\hline F19982 & $\begin{array}{l}\text { Other psychoactive substance use, unspecified with psychoactive substance-induced sleep } \\
\text { disorder }\end{array}$ \\
\hline F19988 & $\begin{array}{l}\text { Other psychoactive substance use, unspecified with other psychoactive substance-induced } \\
\text { disorder }\end{array}$ \\
\hline F1999 & $\begin{array}{l}\text { Other psychoactive substance use, unspecified with unspecified psychoactive substance- } \\
\text { induced disorder }\end{array}$ \\
\hline F1510 & Other stimulant abuse, uncomplicated \\
\hline F15120 & Other stimulant abuse with intoxication, uncomplicated \\
\hline F15121 & Other stimulant abuse with intoxication delirium \\
\hline F15122 & Other stimulant abuse with intoxication with perceptual disturbance \\
\hline F15129 & Other stimulant abuse with intoxication, unspecified \\
\hline F1514 & Other stimulant abuse with stimulant-induced mood disorder \\
\hline F15150 & Other stimulant abuse with stimulant-induced psychotic disorder with delusions \\
\hline F15151 & Other stimulant abuse with stimulant-induced psychotic disorder with hallucinations \\
\hline F15159 & Other stimulant abuse with stimulant-induced psychotic disorder, unspecified \\
\hline F15180 & Other stimulant abuse with stimulant-induced anxiety disorder \\
\hline F15181 & Other stimulant abuse with stimulant-induced sexual dysfunction \\
\hline F15182 & Other stimulant abuse with stimulant-induced sleep disorder \\
\hline F15188 & Other stimulant abuse with other stimulant-induced disorder \\
\hline F1519 & Other stimulant abuse with unspecified stimulant-induced disorder \\
\hline F1520 & Other stimulant dependence, uncomplicated \\
\hline F1521 & Other stimulant dependence, in remission \\
\hline F15220 & Other stimulant dependence with intoxication, uncomplicated \\
\hline F15221 & Other stimulant dependence with intoxication delirium \\
\hline F15222 & Other stimulant dependence with intoxication with perceptual disturbance \\
\hline F15229 & Other stimulant dependence with intoxication, unspecified \\
\hline F1523 & Other stimulant dependence with withdrawal \\
\hline F1524 & Other stimulant dependence with stimulant-induced mood disorder \\
\hline F15250 & Other stimulant dependence with stimulant-induced psychotic disorder with delusions \\
\hline F15251 & Other stimulant dependence with stimulant-induced psychotic disorder with hallucinations \\
\hline F15259 & Other stimulant dependence with stimulant-induced psychotic disorder, unspecified \\
\hline F15280 & Other stimulant dependence with stimulant-induced anxiety disorder \\
\hline F15281 & Other stimulant dependence with stimulant-induced sexual dysfunction \\
\hline F15282 & Other stimulant dependence with stimulant-induced sleep disorder \\
\hline F15288 & Other stimulant dependence with other stimulant-induced disorder \\
\hline F1529 & Other stimulant dependence with unspecified stimulant-induced disorder \\
\hline F1590 & Other stimulant use, unspecified, uncomplicated \\
\hline F15920 & Other stimulant use, unspecified with intoxication, uncomplicated \\
\hline F15921 & Other stimulant use, unspecified with intoxication delirium \\
\hline F15922 & Other stimulant use, unspecified with intoxication with perceptual disturbance \\
\hline F15929 & Other stimulant use, unspecified with intoxication, unspecified \\
\hline
\end{tabular}




\begin{tabular}{|c|c|}
\hline F1593 & Other stimulant use, unspecified with withdrawal \\
\hline F1594 & Other stimulant use, unspecified with stimulant-induced mood disorder \\
\hline F15950 & Other stimulant use, unspecified with stimulant-induced psychotic disorder with delusions \\
\hline F15951 & $\begin{array}{l}\text { Other stimulant use, unspecified with stimulant-induced psychotic disorder with } \\
\text { hallucinations }\end{array}$ \\
\hline F15959 & Other stimulant use, unspecified with stimulant-induced psychotic disorder, unspecified \\
\hline F15980 & Other stimulant use, unspecified with stimulant-induced anxiety disorder \\
\hline F15981 & Other stimulant use, unspecified with stimulant-induced sexual dysfunction \\
\hline F15982 & Other stimulant use, unspecified with stimulant-induced sleep disorder \\
\hline F15988 & Other stimulant use, unspecified with other stimulant-induced disorder \\
\hline F1599 & Other stimulant use, unspecified with unspecified stimulant-induced disorder \\
\hline F550 & Abuse of antacids \\
\hline F551 & Abuse of herbal or folk remedies \\
\hline F552 & Abuse of laxatives \\
\hline F553 & Abuse of steroids or hormones \\
\hline F554 & Abuse of vitamins \\
\hline F558 & Abuse of other non-psychoactive substances \\
\hline 099320 & Drug use complicating pregnancy, unspecified trimester \\
\hline 099321 & Drug use complicating pregnancy, first trimester \\
\hline 099322 & Drug use complicating pregnancy, second trimester \\
\hline 099323 & Drug use complicating pregnancy, third trimester \\
\hline 099324 & Drug use complicating childbirth \\
\hline 099325 & Drug use complicating the puerperium \\
\hline F4310 & Post-traumatic stress disorder, unspecified \\
\hline F4311 & Post-traumatic stress disorder, acute \\
\hline F4312 & Post-traumatic stress disorder, chronic \\
\hline F13121 & Sedative, hypnotic or anxiolytic abuse with intoxication delirium \\
\hline F13129 & Sedative, hypnotic or anxiolytic abuse with intoxication, unspecified \\
\hline F1314 & $\begin{array}{l}\text { Sedative, hypnotic or anxiolytic abuse with sedative, hypnotic or anxiolytic-induced mood } \\
\text { disorder }\end{array}$ \\
\hline F13150 & $\begin{array}{l}\text { Sedative, hypnotic or anxiolytic abuse with sedative, hypnotic or anxiolytic-induced } \\
\text { psychotic disorder with delusions }\end{array}$ \\
\hline F13151 & $\begin{array}{l}\text { Sedative, hypnotic or anxiolytic abuse with sedative, hypnotic or anxiolytic-induced } \\
\text { psychotic disorder with hallucinations }\end{array}$ \\
\hline F13159 & $\begin{array}{l}\text { Sedative, hypnotic or anxiolytic abuse with sedative, hypnotic or anxiolytic-induced } \\
\text { psychotic disorder, unspecified }\end{array}$ \\
\hline F13180 & $\begin{array}{l}\text { Sedative, hypnotic or anxiolytic abuse with sedative, hypnotic or anxiolytic-induced anxiety } \\
\text { disorder }\end{array}$ \\
\hline F13181 & $\begin{array}{l}\text { Sedative, hypnotic or anxiolytic abuse with sedative, hypnotic or anxiolytic-induced sexual } \\
\text { dysfunction }\end{array}$ \\
\hline F13182 & $\begin{array}{l}\text { Sedative, hypnotic or anxiolytic abuse with sedative, hypnotic or anxiolytic-induced sleep } \\
\text { disorder }\end{array}$ \\
\hline F13188 & $\begin{array}{l}\text { Sedative, hypnotic or anxiolytic abuse with other sedative, hypnotic or anxiolytic-induced } \\
\text { disorder }\end{array}$ \\
\hline
\end{tabular}




\begin{tabular}{|c|c|}
\hline F1319 & $\begin{array}{l}\text { Sedative, hypnotic or anxiolytic abuse with unspecified sedative, hypnotic or anxiolytic- } \\
\text { induced disorder }\end{array}$ \\
\hline F13220 & Sedative, hypnotic or anxiolytic dependence with intoxication, uncomplicated \\
\hline F13221 & Sedative, hypnotic or anxiolytic dependence with intoxication delirium \\
\hline F13229 & Sedative, hypnotic or anxiolytic dependence with intoxication, unspecified \\
\hline F13230 & Sedative, hypnotic or anxiolytic dependence with withdrawal, uncomplicated \\
\hline F13231 & Sedative, hypnotic or anxiolytic dependence with withdrawal delirium \\
\hline F13232 & Sedative, hypnotic or anxiolytic dependence with withdrawal with perceptual disturbance \\
\hline F13239 & Sedative, hypnotic or anxiolytic dependence with withdrawal, unspecified \\
\hline F1324 & $\begin{array}{l}\text { Sedative, hypnotic or anxiolytic dependence with sedative, hypnotic or anxiolytic-induced } \\
\text { mood disorder }\end{array}$ \\
\hline F13250 & $\begin{array}{l}\text { Sedative, hypnotic or anxiolytic dependence with sedative, hypnotic or anxiolytic-induced } \\
\text { psychotic disorder with delusions }\end{array}$ \\
\hline F13251 & $\begin{array}{l}\text { Sedative, hypnotic or anxiolytic dependence with sedative, hypnotic or anxiolytic-induced } \\
\text { psychotic disorder with hallucinations }\end{array}$ \\
\hline F13259 & $\begin{array}{l}\text { Sedative, hypnotic or anxiolytic dependence with sedative, hypnotic or anxiolytic-induced } \\
\text { psychotic disorder, unspecified }\end{array}$ \\
\hline F1326 & $\begin{array}{l}\text { Sedative, hypnotic or anxiolytic dependence with sedative, hypnotic or anxiolytic-induced } \\
\text { persisting amnestic disorder }\end{array}$ \\
\hline F1327 & $\begin{array}{l}\text { Sedative, hypnotic or anxiolytic dependence with sedative, hypnotic or anxiolytic-induced } \\
\text { persisting dementia }\end{array}$ \\
\hline F13280 & $\begin{array}{l}\text { Sedative, hypnotic or anxiolytic dependence with sedative, hypnotic or anxiolytic-induced } \\
\text { anxiety disorder }\end{array}$ \\
\hline F13281 & $\begin{array}{l}\text { Sedative, hypnotic or anxiolytic dependence with sedative, hypnotic or anxiolytic-induced } \\
\text { sexual dysfunction }\end{array}$ \\
\hline F13282 & $\begin{array}{l}\text { Sedative, hypnotic or anxiolytic dependence with sedative, hypnotic or anxiolytic-induced } \\
\text { sleep disorder }\end{array}$ \\
\hline F13288 & $\begin{array}{l}\text { Sedative, hypnotic or anxiolytic dependence with other sedative, hypnotic or anxiolytic- } \\
\text { induced disorder }\end{array}$ \\
\hline F1329 & $\begin{array}{l}\text { Sedative, hypnotic or anxiolytic dependence with unspecified sedative, hypnotic or } \\
\text { anxiolytic-induced disorder }\end{array}$ \\
\hline F13920 & Sedative, hypnotic or anxiolytic use, unspecified with intoxication, uncomplicated \\
\hline F13921 & Sedative, hypnotic or anxiolytic use, unspecified with intoxication delirium \\
\hline F13929 & Sedative, hypnotic or anxiolytic use, unspecified with intoxication, unspecified \\
\hline F13930 & Sedative, hypnotic or anxiolytic use, unspecified with withdrawal, uncomplicated \\
\hline F13931 & Sedative, hypnotic or anxiolytic use, unspecified with withdrawal delirium \\
\hline F13932 & $\begin{array}{l}\text { Sedative, hypnotic or anxiolytic use, unspecified with withdrawal with perceptual } \\
\text { disturbances }\end{array}$ \\
\hline F13939 & Sedative, hypnotic or anxiolytic use, unspecified with withdrawal, unspecified \\
\hline F1394 & $\begin{array}{l}\text { Sedative, hypnotic or anxiolytic use, unspecified with sedative, hypnotic or anxiolytic- } \\
\text { induced mood disorder }\end{array}$ \\
\hline F13950 & $\begin{array}{l}\text { Sedative, hypnotic or anxiolytic use, unspecified with sedative, hypnotic or anxiolytic- } \\
\text { induced psychotic disorder with delusions }\end{array}$ \\
\hline F13951 & $\begin{array}{l}\text { Sedative, hypnotic or anxiolytic use, unspecified with sedative, hypnotic or anxiolytic- } \\
\text { induced psychotic disorder with hallucinations }\end{array}$ \\
\hline F13959 & $\begin{array}{l}\text { Sedative, hypnotic or anxiolytic use, unspecified with sedative, hypnotic or anxiolytic- } \\
\text { induced psychotic disorder, unspecified }\end{array}$ \\
\hline
\end{tabular}




\begin{tabular}{|c|c|}
\hline F1396 & $\begin{array}{l}\text { Sedative, hypnotic or anxiolytic use, unspecified with sedative, hypnotic or anxiolytic- } \\
\text { induced persisting amnestic disorder }\end{array}$ \\
\hline F1397 & $\begin{array}{l}\text { Sedative, hypnotic or anxiolytic use, unspecified with sedative, hypnotic or anxiolytic- } \\
\text { induced persisting dementia }\end{array}$ \\
\hline F13980 & $\begin{array}{l}\text { Sedative, hypnotic or anxiolytic use, unspecified with sedative, hypnotic or anxiolytic- } \\
\text { induced anxiety disorder }\end{array}$ \\
\hline F13981 & $\begin{array}{l}\text { Sedative, hypnotic or anxiolytic use, unspecified with sedative, hypnotic or anxiolytic- } \\
\text { induced sexual dysfunction }\end{array}$ \\
\hline F13982 & $\begin{array}{l}\text { Sedative, hypnotic or anxiolytic use, unspecified with sedative, hypnotic or anxiolytic- } \\
\text { induced sleep disorder }\end{array}$ \\
\hline F13988 & $\begin{array}{l}\text { Sedative, hypnotic or anxiolytic use, unspecified with other sedative, hypnotic or anxiolytic- } \\
\text { induced disorder }\end{array}$ \\
\hline F1399 & $\begin{array}{l}\text { Sedative, hypnotic or anxiolytic use, unspecified with unspecified sedative, hypnotic or } \\
\text { anxiolytic-induced disorder }\end{array}$ \\
\hline T1491 & Suicide attempt \\
\hline T1491XA & Suicide attempt, initial encounter \\
\hline T1491XD & Suicide attempt, subsequent encounter \\
\hline T1491XS & Suicide attempt, sequelae \\
\hline T39012A & Poisoning by aspirin, intentional self-harm, initial encounter \\
\hline T39012D & Poisoning by aspirin, intentional self-harm, subsequent encounter \\
\hline T39012S & Poisoning by aspirin, intentional self-harm, sequelae \\
\hline T39092A & Poisoning by salicylates, intentional self-harm, initial encounter \\
\hline T39092D & Poisoning by salicylates, intentional self-harm, subsequent encounter \\
\hline T39092S & Poisoning by salicylates, intentional self-harm, sequelae \\
\hline $\mathrm{T} 391 \times 2 \mathrm{~A}$ & Poisoning by 4-Aminophenol derivatives, intentional self-harm, initial encounter \\
\hline T391X2D & Poisoning by 4-Aminophenol derivatives, intentional self-harm, subsequent encounter \\
\hline T391X2S & Poisoning by 4-Aminophenol derivatives, intentional self-harm, sequelae \\
\hline T392X2A & Poisoning by pyrazolone derivatives, intentional self-harm, initial encounter \\
\hline T392X2D & Poisoning by pyrazolone derivatives, intentional self-harm, subsequent encounter \\
\hline $\mathrm{T} 392 \times 2 \mathrm{~S}$ & Poisoning by pyrazolone derivatives, intentional self-harm, sequelae \\
\hline T39312A & Poisoning by propionic acid derivatives, intentional self-harm, initial encounter \\
\hline T39312D & Poisoning by propionic acid derivatives, intentional self-harm, subsequent encounter \\
\hline T39312S & Poisoning by propionic acid derivatives, intentional self-harm, sequelae \\
\hline T39392A & $\begin{array}{l}\text { Poisoning by other nonsteroidal anti-inflammatory drugs [NSAID], intentional self-harm, } \\
\text { initial encounter }\end{array}$ \\
\hline T39392D & $\begin{array}{l}\text { Poisoning by other nonsteroidal anti-inflammatory drugs [NSAID], intentional self-harm, } \\
\text { subsequent encounter }\end{array}$ \\
\hline T39392S & $\begin{array}{l}\text { Poisoning by other nonsteroidal anti-inflammatory drugs [NSAID], intentional self-harm, } \\
\text { sequelae }\end{array}$ \\
\hline T394X2A & $\begin{array}{l}\text { Poisoning by antirheumatics, not elsewhere classified, intentional self-harm, initial } \\
\text { encounter }\end{array}$ \\
\hline T394X2D & $\begin{array}{l}\text { Poisoning by antirheumatics, not elsewhere classified, intentional self-harm, subsequent } \\
\text { encounter }\end{array}$ \\
\hline $\mathrm{T} 394 \times 2 \mathrm{~S}$ & Poisoning by antirheumatics, not elsewhere classified, intentional self-harm, sequelae \\
\hline
\end{tabular}




\begin{tabular}{|c|c|}
\hline T398X2A & $\begin{array}{l}\text { Poisoning by other nonopioid analgesics and antipyretics, not elsewhere classified, } \\
\text { intentional self-harm, initial encounter }\end{array}$ \\
\hline T398X2D & $\begin{array}{l}\text { Poisoning by other nonopioid analgesics and antipyretics, not elsewhere classified, } \\
\text { intentional self-harm, subsequent encounter }\end{array}$ \\
\hline $\mathrm{T} 398 \times 2 \mathrm{~S}$ & $\begin{array}{l}\text { Poisoning by other nonopioid analgesics and antipyretics, not elsewhere classified, } \\
\text { intentional self-harm, sequelae }\end{array}$ \\
\hline T3992XA & $\begin{array}{l}\text { Poisoning by unspecified nonopioid analgesic, antipyretic and antirheumatic, intentional } \\
\text { self-harm, initial encounter }\end{array}$ \\
\hline T3992XD & $\begin{array}{l}\text { Poisoning by unspecified nonopioid analgesic, antipyretic and antirheumatic, intentional } \\
\text { self-harm, subsequent encounter }\end{array}$ \\
\hline T3992XS & $\begin{array}{l}\text { Poisoning by unspecified nonopioid analgesic, antipyretic and antirheumatic, intentional } \\
\text { self-harm, sequelae }\end{array}$ \\
\hline $\mathrm{T} 400 \times 2 \mathrm{~A}$ & Poisoning by opium, intentional self-harm, initial encounter \\
\hline T400X2D & Poisoning by opium, intentional self-harm, subsequent encounter \\
\hline $\mathrm{T} 400 \times 2 \mathrm{~S}$ & Poisoning by opium, intentional self-harm, sequelae \\
\hline T401X2A & Poisoning by heroin, intentional self-harm, initial encounter \\
\hline $\mathrm{T} 401 \mathrm{X} 2 \mathrm{D}$ & Poisoning by heroin, intentional self-harm, subsequent encounter \\
\hline $\mathrm{T} 401 \mathrm{X} 2 \mathrm{~S}$ & Poisoning by heroin, intentional self-harm, sequelae \\
\hline T402X2A & Poisoning by other opioids, intentional self-harm, initial encounter \\
\hline $\mathrm{T} 402 \times 2 \mathrm{D}$ & Poisoning by other opioids, intentional self-harm, subsequent encounter \\
\hline T402X2S & Poisoning by other opioids, intentional self-harm, sequelae \\
\hline $\mathrm{T} 403 \times 2 \mathrm{~A}$ & Poisoning by methadone, intentional self-harm, initial encounter \\
\hline T403X2D & Poisoning by methadone, intentional self-harm, subsequent encounter \\
\hline $\mathrm{T} 403 \times 2 \mathrm{~S}$ & Poisoning by methadone, intentional self-harm, sequelae \\
\hline T404X2A & Poisoning by other synthetic narcotics, intentional self-harm, initial encounter \\
\hline $\mathrm{T} 404 \mathrm{X} 2 \mathrm{D}$ & Poisoning by other synthetic narcotics, intentional self-harm, subsequent encounter \\
\hline $\mathrm{T} 404 \times 2 \mathrm{~S}$ & Poisoning by other synthetic narcotics, intentional self-harm, sequelae \\
\hline $\mathrm{T} 405 \mathrm{X} 2 \mathrm{~A}$ & Poisoning by cocaine, intentional self-harm, initial encounter \\
\hline $\mathrm{T} 405 \mathrm{X} 2 \mathrm{D}$ & Poisoning by cocaine, intentional self-harm, subsequent encounter \\
\hline T405X2S & Poisoning by cocaine, intentional self-harm, sequelae \\
\hline T40602A & Poisoning by unspecified narcotics, intentional self-harm, initial encounter \\
\hline T40602D & Poisoning by unspecified narcotics, intentional self-harm, subsequent encounter \\
\hline T40602S & Poisoning by unspecified narcotics, intentional self-harm, sequelae \\
\hline T40692A & Poisoning by other narcotics, intentional self-harm, initial encounter \\
\hline T40692D & Poisoning by other narcotics, intentional self-harm, subsequent encounter \\
\hline T40692S & Poisoning by other narcotics, intentional self-harm, sequelae \\
\hline T407X2A & Poisoning by cannabis (derivatives), intentional self-harm, initial encounter \\
\hline $\mathrm{T} 407 \mathrm{X} 2 \mathrm{D}$ & Poisoning by cannabis (derivatives), intentional self-harm, subsequent encounter \\
\hline $\mathrm{T} 407 \times 2 \mathrm{~S}$ & Poisoning by cannabis (derivatives), intentional self-harm, sequelae \\
\hline T408X2A & Poisoning by lysergide [LSD], intentional self-harm, initial encounter \\
\hline $\mathrm{T} 408 \mathrm{X} 2 \mathrm{D}$ & Poisoning by lysergide [LSD], intentional self-harm, subsequent encounter \\
\hline $\mathrm{T} 408 \times 2 \mathrm{~S}$ & Poisoning by lysergide [LSD], intentional self-harm, sequelae \\
\hline
\end{tabular}




\begin{tabular}{|c|c|}
\hline T40902A & $\begin{array}{l}\text { Poisoning by unspecified psychodysleptics [hallucinogens], intentional self-harm, initial } \\
\text { encounter }\end{array}$ \\
\hline T40902D & $\begin{array}{l}\text { Poisoning by unspecified psychodysleptics [hallucinogens], intentional self-harm, } \\
\text { subsequent encounter }\end{array}$ \\
\hline $\mathrm{T} 40902 \mathrm{~S}$ & Poisoning by unspecified psychodysleptics [hallucinogens], intentional self-harm, sequelae \\
\hline T40992A & Poisoning by other psychodysleptics [hallucinogens], intentional self-harm, initial encounter \\
\hline T40992D & $\begin{array}{l}\text { Poisoning by other psychodysleptics [hallucinogens], intentional self-harm, subsequent } \\
\text { encounter }\end{array}$ \\
\hline T40992S & Poisoning by other psychodysleptics [hallucinogens], intentional self-harm, sequelae \\
\hline $\mathrm{T} 423 \times 2 \mathrm{~A}$ & Poisoning by barbiturates, intentional self-harm, initial encounter \\
\hline $\mathrm{T} 423 \mathrm{X} 2 \mathrm{D}$ & Poisoning by barbiturates, intentional self-harm, subsequent encounter \\
\hline $\mathrm{T} 423 \times 2 \mathrm{~S}$ & Poisoning by barbiturates, intentional self-harm, sequelae \\
\hline $\mathrm{T} 424 \mathrm{X} 2 \mathrm{~A}$ & Poisoning by benzodiazepines, intentional self-harm, initial encounter \\
\hline T424X2D & Poisoning by benzodiazepines, intentional self-harm, subsequent encounter \\
\hline $\mathrm{T} 424 \mathrm{X} 2 \mathrm{~S}$ & Poisoning by benzodiazepines, intentional self-harm, sequelae \\
\hline T4272XA & $\begin{array}{l}\text { Poisoning by unspecified antiepileptic and sedative-hypnotic drugs, intentional self-harm, } \\
\text { initial encounter }\end{array}$ \\
\hline 2XD & $\begin{array}{l}\text { Poisoning by unspecified antiepileptic and sedative-hypnotic drugs, intentional self-harm, } \\
\text { subsequent encounter }\end{array}$ \\
\hline T4272XS & $\begin{array}{l}\text { Poisoning by unspecified antiepileptic and sedative-hypnotic drugs, intentional self-harm, } \\
\text { sequela }\end{array}$ \\
\hline T43012A & Poisoning by tricyclic antidepressants, intentional self-harm, initial encounter \\
\hline T43012D & Poisoning by tricyclic antidepressants, intentional self-harm, subsequent encounter \\
\hline T43012S & idepressants, intentional self-harm, sequelae \\
\hline T43022A & Poisoning by tetracyclic antidepressants, intentional self-harm, initial encounter \\
\hline T43022D & Poisoning by tetracyclic antidepressants, intentional self-harm, subsequent encounter \\
\hline T43022S & Poisoning by tetracyclic antidepressants, intentional self-harm, sequelae \\
\hline $\mathrm{X} 2 \mathrm{~A}$ & $\begin{array}{l}\text { Poisoning by monoamine-oxidase-inhibitor antidepressants, intentional self-harm, initial } \\
\text { encounter }\end{array}$ \\
\hline T431X2D & $\begin{array}{l}\text { Poisoning by monoamine-oxidase-inhibitor antidepressants, intentional self-harm, } \\
\text { subsequent encounter }\end{array}$ \\
\hline $\mathrm{T} 431 \mathrm{X} 2 \mathrm{~S}$ & Poisoning by monoamine-oxidase-inhibitor antidepressants, intentional self-harm, se \\
\hline T43202A & Poisoning by unspecified antidepressants, intentional self-harm, initial encounter \\
\hline T43202D & Poisoning by unspecified antidepressants, intentional self-harm, subsequent encounter \\
\hline T43202S & Poisoning by unspecified antidepressants, intentional self-harm, sequelae \\
\hline T43212A & $\begin{array}{l}\text { Poisoning by selective serotonin and norepinephrine reuptake inhibitors, intentional self- } \\
\text { harm, initial encounter }\end{array}$ \\
\hline T43212D & $\begin{array}{l}\text { Poisoning by selective serotonin and norepinephrine reuptake inhibitors, intentional self- } \\
\text { harm, subsequent encounter }\end{array}$ \\
\hline T43212S & $\begin{array}{l}\text { Poisoning by selective serotonin and norepinephrine reuptake inhibitors, intentional self- } \\
\text { harm, sequela }\end{array}$ \\
\hline T43222A & Poisoning by selective serotonin reuptake inhibitors, intentional self-harm, initial encounter \\
\hline $\mathrm{T} 43222 \mathrm{D}$ & $\begin{array}{l}\text { Poisoning by selective serotonin reuptake inhibitors, intentional self-harm, subsequent } \\
\text { encounter }\end{array}$ \\
\hline T43222S & y selective serotonin reuptake inhibitors, intentional self-harm, sequelae \\
\hline
\end{tabular}




\begin{tabular}{|c|c|}
\hline T43292A & Poisoning by other antidepressants, intentional self-harm, initial encounter \\
\hline T43292D & Poisoning by other antidepressants, intentional self-harm, subsequent encounter \\
\hline T43292S & Poisoning by other antidepressants, intentional self-harm, sequelae \\
\hline $\mathrm{T} 433 \times 2 \mathrm{~A}$ & $\begin{array}{l}\text { Poisoning by phenothiazine antipsychotics and neuroleptics, intentional self-harm, initial } \\
\text { encounter }\end{array}$ \\
\hline $\mathrm{T} 433 \times 2 \mathrm{D}$ & $\begin{array}{l}\text { Poisoning by phenothiazine antipsychotics and neuroleptics, intentional self-harm, } \\
\text { subsequent encounter }\end{array}$ \\
\hline $\mathrm{T} 433 \times 2 \mathrm{~S}$ & Poisoning by phenothiazine antipsychotics and neuroleptics, intentional self-harm, sequelae \\
\hline $\mathrm{T} 434 \mathrm{X} 2 \mathrm{~A}$ & $\begin{array}{l}\text { Poisoning by butyrophenone and thiothixene neuroleptics, intentional self-harm, initial } \\
\text { encounter }\end{array}$ \\
\hline $\mathrm{T} 434 \mathrm{X} 2 \mathrm{D}$ & $\begin{array}{l}\text { Poisoning by butyrophenone and thiothixene neuroleptics, intentional self-harm, } \\
\text { subsequent encounter }\end{array}$ \\
\hline $\mathrm{T} 434 \mathrm{X} 2 \mathrm{~S}$ & Poisoning by butyrophenone and thiothixene neuroleptics, intentional self-harm, sequelae \\
\hline $\mathrm{T} 43502 \mathrm{~A}$ & $\begin{array}{l}\text { Poisoning by unspecified antipsychotics and neuroleptics, intentional self-harm, initial } \\
\text { encounter }\end{array}$ \\
\hline T43502D & $\begin{array}{l}\text { Poisoning by unspecified antipsychotics and neuroleptics, intentional self-harm, subsequent } \\
\text { encounter }\end{array}$ \\
\hline T43502S & Poisoning by unspecified antipsychotics and neuroleptics, intentional self-harm, sequelae \\
\hline T43592A & Poisoning by other antipsychotics and neuroleptics, intentional self-harm, initial encounter \\
\hline T43592D & $\begin{array}{l}\text { Poisoning by other antipsychotics and neuroleptics, intentional self-harm, subsequent } \\
\text { encounter }\end{array}$ \\
\hline T43592S & Poisoning by other antipsychotics and neuroleptics, intentional self-harm, sequelae \\
\hline T43602A & Poisoning by unspecified psychostimulants, intentional self-harm, initial encounter \\
\hline T43602D & Poisoning by unspecified psychostimulants, intentional self-harm, subsequent encounter \\
\hline T43602S & Poisoning by unspecified psychostimulants, intentional self-harm, sequelae \\
\hline T43612A & Poisoning by caffeine, intentional self-harm, initial encounter \\
\hline T43612D & Poisoning by caffeine, intentional self-harm, subsequent encounter \\
\hline T43612S & Poisoning by caffeine, intentional self-harm, sequelae \\
\hline T43622A & Poisoning by amphetamines, intentional self-harm, initial encounter \\
\hline T43622D & Poisoning by amphetamines, intentional self-harm, subsequent encounter \\
\hline T43622S & Poisoning by amphetamines, intentional self-harm, sequelae \\
\hline T43632A & Poisoning by methylphenidate, intentional self-harm, initial encounter \\
\hline T43632D & Poisoning by methylphenidate, intentional self-harm, subsequent encounter \\
\hline T43632S & Poisoning by methylphenidate, intentional self-harm, sequelae \\
\hline T43692A & Poisoning by other psychostimulants, intentional self-harm, initial encounter \\
\hline T43692D & Poisoning by other psychostimulants, intentional self-harm, subsequent encounter \\
\hline T43692S & Poisoning by other psychostimulants, intentional self-harm, sequelae \\
\hline $\mathrm{T} 438 \times 2 \mathrm{~A}$ & Poisoning by other psychotropic drugs, intentional self-harm, initial encounter \\
\hline $\mathrm{T} 438 \times 2 \mathrm{D}$ & Poisoning by other psychotropic drugs, intentional self-harm, subsequent encounter \\
\hline $\mathrm{T} 438 \times 2 \mathrm{~S}$ & Poisoning by other psychotropic drugs, intentional self-harm, sequelae \\
\hline T4392XA & Poisoning by unspecified psychotropic drug, intentional self-harm, initial encounter \\
\hline T4392XD & Poisoning by unspecified psychotropic drug, intentional self-harm, subsequent encounter \\
\hline T4392XS & Poisoning by unspecified psychotropic drug, intentional self-harm, sequelae \\
\hline
\end{tabular}




\begin{tabular}{|c|c|}
\hline T50902A & $\begin{array}{l}\text { Poisoning by unspecified drugs, medicaments and biological substances, intentional self- } \\
\text { harm, initial encounter }\end{array}$ \\
\hline T50902D & $\begin{array}{l}\text { Poisoning by unspecified drugs, medicaments and biological substances, intentional self- } \\
\text { harm, subsequent encounter }\end{array}$ \\
\hline T50902S & $\begin{array}{l}\text { Poisoning by unspecified drugs, medicaments and biological substances, intentional self- } \\
\text { harm, sequelae }\end{array}$ \\
\hline T50992A & $\begin{array}{l}\text { Poisoning by other drugs, medicaments and biological substances, intentional self-harm, } \\
\text { initial encounter }\end{array}$ \\
\hline T50992D & $\begin{array}{l}\text { Poisoning by other drugs, medicaments and biological substances, intentional self-harm, } \\
\text { subsequent encounter }\end{array}$ \\
\hline T50992S & $\begin{array}{l}\text { Poisoning by other drugs, medicaments and biological substances, intentional self-harm, } \\
\text { sequelae }\end{array}$ \\
\hline T5802XA & $\begin{array}{l}\text { Toxic effect of carbon monoxide from motor vehicle exhaust, intentional self-harm, initial } \\
\text { encounter }\end{array}$ \\
\hline T5802XD & $\begin{array}{l}\text { Toxic effect of carbon monoxide from motor vehicle exhaust, intentional self-harm, } \\
\text { subsequent encounter }\end{array}$ \\
\hline T5802XS & $\begin{array}{l}\text { Toxic effect of carbon monoxide from motor vehicle exhaust, intentional self-harm, } \\
\text { sequelae }\end{array}$ \\
\hline T5812XA & Toxic effect of carbon monoxide from utility gas, intentional self-harm, initial encounter \\
\hline T5812XD & $\begin{array}{l}\text { Toxic effect of carbon monoxide from utility gas, intentional self-harm, subsequent } \\
\text { encounter }\end{array}$ \\
\hline T5812XS & Toxic effect of carbon monoxide from utility gas, intentional self-harm, sequelae \\
\hline T582X2A & $\begin{array}{l}\text { Toxic effect of carbon monoxide from incomplete combustion of other domestic fuels, } \\
\text { intentional self-harm, initial encounter }\end{array}$ \\
\hline $\mathrm{T} 582 \times 2 \mathrm{D}$ & $\begin{array}{l}\text { Toxic effect of carbon monoxide from incomplete combustion of other domestic fuels, } \\
\text { intentional self-harm, subsequent encounter }\end{array}$ \\
\hline $\mathrm{T} 582 \mathrm{X} 2 \mathrm{~S}$ & $\begin{array}{l}\text { Toxic effect of carbon monoxide from incomplete combustion of other domestic fuels, } \\
\text { intentional self-harm, sequelae }\end{array}$ \\
\hline $\mathrm{T} 588 \mathrm{X} 2 \mathrm{~A}$ & Toxic effect of carbon monoxide from other source, intentional self-harm, initial encounter \\
\hline T588X2D & $\begin{array}{l}\text { Toxic effect of carbon monoxide from other source, intentional self-harm, subsequent } \\
\text { encounter }\end{array}$ \\
\hline T588X2S & Toxic effect of carbon monoxide from other source, intentional self-harm, sequelae \\
\hline T5892XA & $\begin{array}{l}\text { Toxic effect of carbon monoxide from unspecified source, intentional self-harm, initial } \\
\text { encounter }\end{array}$ \\
\hline T5892XD & $\begin{array}{l}\text { Toxic effect of carbon monoxide from unspecified source, intentional self-harm, subsequent } \\
\text { encounter }\end{array}$ \\
\hline T58 & Toxic effect of carbon monoxide from unspecified source, intentional self-harm, sequelae \\
\hline X710XXA & nersion while in bathtub, initial encounter \\
\hline X710XXD & Intentional self-harm by drowning and submersion while in bathtub, subsequent encounter \\
\hline X710XXS & Intentional self-harm by drowning and submersion while in bathtub, sequelae \\
\hline X711XXA & Intentional self-harm by drowning and submersion while in swimming pool, initial encounter \\
\hline X711XXD & $\begin{array}{l}\text { Intentional self-harm by drowning and submersion while in swimming pool, subsequent } \\
\text { encounter }\end{array}$ \\
\hline X711XXS & Intentional self-harm by drowning and submersion while in swimming pool, sequelae \\
\hline X712XXA & $\begin{array}{l}\text { Intentional self-harm by drowning and submersion after jump into swimming pool, initial } \\
\text { encounter }\end{array}$ \\
\hline X712XXD & $\begin{array}{l}\text { Intentional self-harm by drowning and submersion after jump into swimming pool, } \\
\text { subsequent encounter }\end{array}$ \\
\hline
\end{tabular}




\begin{tabular}{|c|c|}
\hline X712XXS & Intentional self-harm by drowning and submersion after jump into swimming pool, sequela \\
\hline X713XXA & Intentional self-harm by drowning and submersion in natural water, initial encounter \\
\hline X713XXD & Intentional self-harm by drowning and submersion in natural water, subsequent encounter \\
\hline X713XXS & Intentional self-harm by drowning and submersion in natural water, sequelae \\
\hline X718XXA & Other intentional self-harm by drowning and submersion, initial encounter \\
\hline X718XXD & Other intentional self-harm by drowning and submersion, subsequent encounter \\
\hline X718XXS & Other intentional self-harm by drowning and submersion, sequelae \\
\hline X719XXA & Intentional self-harm by drowning and submersion, unspecified, initial encounter \\
\hline X719XXD & Intentional self-harm by drowning and submersion, unspecified, subsequent encounter \\
\hline X719XXS & Intentional self-harm by drowning and submersion, unspecified, sequelae \\
\hline X72XXXA & Intentional self-harm by handgun discharge, initial encounter \\
\hline X72XXXD & Intentional self-harm by handgun discharge, subsequent encounter \\
\hline X72XXXS & Intentional self-harm by handgun discharge, sequelae \\
\hline X730XXA & Intentional self-harm by shotgun discharge, initial encounter \\
\hline X730XXD & Intentional self-harm by shotgun discharge, subsequent encounter \\
\hline X730XXS & Intentional self-harm by shotgun discharge, sequelae \\
\hline X731XXA & Intentional self-harm by hunting rifle discharge, initial encounter \\
\hline X731XXD & Intentional self-harm by hunting rifle discharge, subsequent encounter \\
\hline X731XXS & Intentional self-harm by hunting rifle discharge, sequelae \\
\hline X732XXA & Intentional self-harm by machine gun discharge, initial encounter \\
\hline X732XXD & Intentional self-harm by machine gun discharge, subsequent encounter \\
\hline X732XXS & Intentional self-harm by machine gun discharge, sequelae \\
\hline X738XXA & Intentional self-harm by other larger firearm discharge, initial encounter \\
\hline X738XXD & Intentional self-harm by other larger firearm discharge, subsequent encounter \\
\hline X738XXs & Intentional self-harm by other larger firearm discharge, sequelae \\
\hline X739XXA & Intentional self-harm by unspecified larger firearm discharge, initial encounter \\
\hline X739XXD & Intentional self-harm by unspecified larger firearm discharge, subsequent encounter \\
\hline X739XXS & Intentional self-harm by unspecified larger firearm discharge, sequelae \\
\hline X7401XA & Intentional self-harm by airgun, initial encounter \\
\hline X7401XD & Intentional self-harm by airgun, subsequent encounter \\
\hline X7401XS & Intentional self-harm by airgun, sequelae \\
\hline X7402XA & Intentional self-harm by paintball gun, initial encounter \\
\hline X7402XD & Intentional self-harm by paintball gun, subsequent encounter \\
\hline X7402XS & Intentional self-harm by paintball gun, sequelae \\
\hline X7409XA & Intentional self-harm by other gas, air or spring-operated gun, initial encounter \\
\hline X7409XD & Intentional self-harm by other gas, air or spring-operated gun, subsequent encounter \\
\hline X7409XS & Intentional self-harm by other gas, air or spring-operated gun, sequelae \\
\hline X748XXA & Intentional self-harm by other firearm discharge, initial encounter \\
\hline X748XXD & Intentional self-harm by other firearm discharge, subsequent encounter \\
\hline X748XXS & Intentional self-harm by other firearm discharge, sequelae \\
\hline
\end{tabular}




\begin{tabular}{|c|c|}
\hline X749XXA & Intentional self-harm by unspecified firearm discharge, initial encounter \\
\hline X749XXD & Intentional self-harm by unspecified firearm discharge, subsequent encounter \\
\hline X749XXS & Intentional self-harm by unspecified firearm discharge, sequelae \\
\hline X75XXXA & Intentional self-harm by explosive material, initial encounter \\
\hline X75XXXD & Intentional self-harm by explosive material, subsequent encounter \\
\hline X75XXXS & Intentional self-harm by explosive material, sequelae \\
\hline X76XXXA & Intentional self-harm by smoke, fire and flames, initial encounter \\
\hline X76XXXD & Intentional self-harm by smoke, fire and flames, subsequent encounter \\
\hline X76XXXS & Intentional self-harm by smoke, fire and flames, sequelae \\
\hline X770XXA & Intentional self-harm by steam or hot vapors, initial encounter \\
\hline X770XXD & Intentional self-harm by steam or hot vapors, subsequent encounter \\
\hline X770XXS & Intentional self-harm by steam or hot vapors, sequelae \\
\hline X771XXA & Intentional self-harm by hot tap water, initial encounter \\
\hline X771XXD & Intentional self-harm by hot tap water, subsequent encounter \\
\hline X771XXS & Intentional self-harm by hot tap water, sequelae \\
\hline X772XXA & Intentional self-harm by other hot fluids, initial encounter \\
\hline X772XXD & Intentional self-harm by other hot fluids, subsequent encounter \\
\hline X772XXS & Intentional self-harm by other hot fluids, sequelae \\
\hline X773XXA & Intentional self-harm by hot household appliances, initial encounter \\
\hline X773XXD & Intentional self-harm by hot household appliances, subsequent encounter \\
\hline X773XXs & Intentional self-harm by hot household appliances, sequelae \\
\hline X778XXA & Intentional self-harm by other hot objects, initial encounter \\
\hline X778XXD & Intentional self-harm by other hot objects, subsequent encounter \\
\hline X778XXS & Intentional self-harm by other hot objects, sequelae \\
\hline X779XXA & Intentional self-harm by unspecified hot objects, initial encounter \\
\hline X779XXD & Intentional self-harm by unspecified hot objects, subsequent encounter \\
\hline X779XXS & Intentional self-harm by unspecified hot objects, sequelae \\
\hline X780XXA & Intentional self-harm by sharp glass, initial encounter \\
\hline X780XXD & Intentional self-harm by sharp glass, subsequent encounter \\
\hline X780XXS & Intentional self-harm by sharp glass, sequelae \\
\hline X781XXA & Intentional self-harm by knife, initial encounter \\
\hline X781XXD & Intentional self-harm by knife, subsequent encounter \\
\hline X781XXS & Intentional self-harm by knife, sequelae \\
\hline X782XXA & Intentional self-harm by sword or dagger, initial encounter \\
\hline X782XXD & Intentional self-harm by sword or dagger, subsequent encounter \\
\hline X782XXS & Intentional self-harm by sword or dagger, sequelae \\
\hline X788XXA & Intentional self-harm by other sharp object, initial encounter \\
\hline X788XXD & Intentional self-harm by other sharp object, subsequent encounter \\
\hline X788XXS & Intentional self-harm by other sharp object, sequelae \\
\hline
\end{tabular}




\begin{tabular}{|c|c|}
\hline X789XXA & Intentional self-harm by unspecified sharp object, initial encounter \\
\hline X789XXD & Intentional self-harm by unspecified sharp object, subsequent encounter \\
\hline X789XXS & Intentional self-harm by unspecified sharp object, sequelae \\
\hline X79XXXA & Intentional self-harm by blunt object, initial encounter \\
\hline X79XXXD & Intentional self-harm by blunt object, subsequent encounter \\
\hline X79XXXS & Intentional self-harm by blunt object, sequelae \\
\hline X80XXXA & Intentional self-harm by jumping from a high place, initial encounter \\
\hline X80XXXD & Intentional self-harm by jumping from a high place, subsequent encounter \\
\hline X80XXXS & Intentional self-harm by jumping from a high place, sequelae \\
\hline X810XXA & Intentional self-harm by jumping or lying in front of motor vehicle, initial encounter \\
\hline X810XXD & Intentional self-harm by jumping or lying in front of motor vehicle, subsequent encounter \\
\hline X810XXS & Intentional self-harm by jumping or lying in front of motor vehicle, sequelae \\
\hline X811XXA & Intentional self-harm by jumping or lying in front of (subway) train, initial encounter \\
\hline X811XXD & Intentional self-harm by jumping or lying in front of (subway) train, subsequent encounter \\
\hline X811XXS & Intentional self-harm by jumping or lying in front of (subway) train, sequelae \\
\hline X818XXA & Intentional self-harm by jumping or lying in front of other moving object, initial encounter \\
\hline X818XXD & $\begin{array}{l}\text { Intentional self-harm by jumping or lying in front of other moving object, subsequent } \\
\text { encounter }\end{array}$ \\
\hline X818XXS & Intentional self-harm by jumping or lying in front of other moving object, sequelae \\
\hline X820XXA & Intentional collision of motor vehicle with other motor vehicle, initial encounter \\
\hline X820XXD & Intentional collision of motor vehicle with other motor vehicle, subsequent encounter \\
\hline X820XXS & Intentional collision of motor vehicle with other motor vehicle, sequelae \\
\hline X821XXA & Intentional collision of motor vehicle with train, initial encounter \\
\hline X821XXD & Intentional collision of motor vehicle with train, subsequent encounter \\
\hline X821XXS & Intentional collision of motor vehicle with train, sequelae \\
\hline X822XXA & Intentional collision of motor vehicle with tree, initial encounter \\
\hline X822XXD & Intentional collision of motor vehicle with tree, subsequent encounter \\
\hline X822XXS & Intentional collision of motor vehicle with tree, sequelae \\
\hline X828XXA & Other intentional self-harm by crashing of motor vehicle, initial encounter \\
\hline X828XXD & Other intentional self-harm by crashing of motor vehicle, subsequent encounter \\
\hline X828XXS & Other intentional self-harm by crashing of motor vehicle, sequelae \\
\hline X830XXA & Intentional self-harm by crashing of aircraft, initial encounter \\
\hline X830XXD & Intentional self-harm by crashing of aircraft, subsequent encounter \\
\hline X830XXS & Intentional self-harm by crashing of aircraft, sequelae \\
\hline X831XXA & Intentional self-harm by electrocution, initial encounter \\
\hline $\mathrm{X} 831 \mathrm{XXD}$ & Intentional self-harm by electrocution, subsequent encounter \\
\hline X831XXS & Intentional self-harm by electrocution, sequelae \\
\hline X832XXA & Intentional self-harm by exposure to extremes of cold, initial encounter \\
\hline $\mathrm{X} 832 \mathrm{XXD}$ & Intentional self-harm by exposure to extremes of cold, subsequent encounter \\
\hline $\mathrm{X} 832 \mathrm{XXS}$ & Intentional self-harm by exposure to extremes of cold, sequelae \\
\hline $\mathrm{X} 838 \mathrm{XXA}$ & Intentional self-harm by other specified means, initial encounter \\
\hline X838XXD & Intentional self-harm by other specified means, subsequent encounter \\
\hline X838XXS & Intentional self-harm by other specified means, sequelae \\
\hline
\end{tabular}

(ICD10Data.com, 2018) 
Appendix M: OUD-Related Infection Codes Queried

\begin{tabular}{|c|c|}
\hline $\begin{array}{l}\text { Infection Related to } \\
\text { Intravenous Drug Use }\end{array}$ & ICD-10 \\
\hline Endocarditis & $133 . x$ \\
\hline Candida endocarditis & b37.6 \\
\hline Osteomyelitis & m86.x \\
\hline Bacteremia & R78.81 \\
\hline Epidural Abscess/ Discitis & m46.4; m51.9; m50.x; m51.8 \\
\hline Septic Arthritis & mo0. \\
\hline Brain Abscess & g06.x \\
\hline Prosthetic Joint Infection & t84.5x \\
\hline Necrotizing fasciitis & m72.6 \\
\hline Empyema & $j 86$ \\
\hline Lung Abscess & j85 \\
\hline
\end{tabular}

Retrieved from: H. Englander, personal communication, January 25, 2018 


\section{Appendix N: Non-OAT Pharmacotherapy Definitions}

\section{Acronyms}

OAT = opioid agonist therapy; HCPCS = Healthcare Common Procedure Coding System .

\section{Pre-Admission}

\section{Pre-Opioid}

All formularies and routes of administration were included as present in the dataset. The preopioid variable included the presence of one of these medications: acetaminophen/codeine, acetaminophen/hydrocodone, acetaminophen/oxycodone, belladonna/opium, chlorpheniramine/hydrocodone, codeine, fentanyl, hydromorphone, meperidine, morphine, oxycodone, oxymorphone, tapentadol, and tramadol.

\section{Pre-Benzodiazepine}

All formularies and routes of administration were included as present in the dataset. The prebenzodiazepine variable included the presence of one of these medications: alprazolam, clonazepam, diazepam, temazepam, and lorazepam.

\section{Pre-Gabapentin/Pregabalin}

All formularies and routes of administration were included as present in the dataset. The variable included the presence of one of these medications: gabapentin or pregabalin.

\section{Pre-Naltrexone}

All formularies and routes of administration were included as present in the dataset. The variable included the presence of the tablet or injectable or HCPCS code J2315.

\section{Pre-Concurrent Opioid and Benzodiazepine}

Cases with both an opioid and benzodiazepine fill in the time period.

\section{Pre-Naloxone}

All formularies and routes of administration were included as present in the dataset (i.e., injection solution, kit, solution nasal spray).

\section{Admission Pharmacotherapy}

\section{Admission}

The majority of inpatient medications reported in this study came from the "Bar Code Medication Administration" table. Outpatient appointments are possible during admission; thus, resulting in an outpatient pharmacy prescription. In this cohort less than $1 \%(5,861$ of 715,277$)$ of medications received during hospitalization were recorded as outpatient prescriptions rather than medication administered.

\section{Admission-Opioid}

All formularies and routes of administration were included as present in the dataset (e.g., tablet, oral solution, injection, suppository, nasal spray). The admission opioid variable included the presence of one of these medications: acetaminophen/codeine, acetaminophen/hydrocodone, 
acetaminophen/oxycodone, belladonna/opium, butorphanol, bupivacaine/fentanyl, codeine, fentanyl, hydromorphone, meperidine, morphine, oxycodone, oxymorphone, tapentadol, and tramadol.

\section{Any Adjuvant Therapy}

The presence of a first line withdrawal management adjuvant or a second-line adjuvant as defined below.

\section{First-Line Adjuvant: Clonidine}

The first-line variable included the presence of clonidine. Patch or tablet formularies were included in the query for the dataset.

\section{Second-Line Adjuvant}

The second-line adjuvant was defined using the VHA recommendations for withdrawal management for OUD (U.S. Department of Veterans Affairs, 2016b). All formularies and routes of administration were included as present in the dataset. The variable included the presence of one of these medications: baclofen, gabapentin, pregabalin, and tizanidine.

\section{Admission-Gabapentin/Pregabalin}

All formularies and routes of administration were included as present in the dataset. The variable included the presence of one of these medications: gabapentin or pregabalin.

\section{Admission-Benzodiazepine}

All formularies and routes of administration were included as present in the dataset. The admission-benzodiazepine variable included the presence of one of these medications: alprazolam, clonazepam, diazepam, midazolam, temazepam, and lorazepam.

\section{Admission-Naltrexone}

All formularies and routes of administration (tablet and injectable) were included as present in the dataset. HCPCS code $\mathrm{J} 2315$ was queried but not present in dataset.

\section{Admission-No Opioid and No OAT}

Cases without an opioid present or OAT present during admission.

\section{Post-Opioids}

\section{Post-Admission}

All formularies and routes of administration (e.g., suppository vs. oral) were included as present in the dataset. The post-opioid variable included the presence of one of these medications: belladonna/opium, chlorpheniramine/hydrocodone, codeine, fentanyl, hydromorphone, meperidine, morphine, oxycodone, oxymorphone, tapentadol, and tramadol.

\section{Post-Gabapentin/Pregabalin}

All formularies and routes of administration were included as present in the dataset. The variable included the presence of one of these medications: gabapentin or pregabalin. 


\section{Post-Benzodiazepine}

All formularies and routes of administration were included as present in the dataset. The postbenzodiazepine variable included the presence of one of these medications: alprazolam, clonazepam, diazepam, midazolam, temazepam, and lorazepam.

\section{Post-Naltrexone}

All formularies and routes of administration were included as present in the dataset. The variable included the presence of the tablet or injectable or HCPCS code J2315.

\section{Post-Naloxone}

All formularies and routes of administration were included as present in the dataset (i.e., injection solution, kit, solution nasal spray).

Post-Concurrent Opioid and Benzodiazepine

Cases with both an opioid AND benzodiazepine fill in the time period. 


\section{Appendix 0: OAT Pharmacotherapy Definitions}

\section{Pre-Admission OAT}

Pre-admission OAT included a visit to an VHA OTP (counted by VHA stop code) or Healthcare Common Procedure Coding System (HCPCS) codes (H0033: non-specific oral medication direct observation; J0574: buprenorphine/naloxone 6.1-10 mg; J0575: buprenorphine/naloxone > 10mg; J0571: oral buprenorphine $1 \mathrm{mg}$; S0109: methadone oral $5 \mathrm{mg}$ ) or outpatient buprenorphine filled prescriptions (only FDA-approved OAT formularies and routes of administration were included: buprenorphine film buccal; buprenorphine sublingual tablet; buprenorphine/naloxone film sublingual; buprenorphine/naloxone sublingual tablet).

\section{Admission OAT}

Admission OAT was defined as a visit to an VHA OTP (counted by VHA stop code) or HCPCS codes (H0033: non-specific oral medication direct observation; J0571: oral buprenorphine $1 \mathrm{mg}$; S0109: methadone oral $5 \mathrm{mg}$ ) or any formulation of buprenorphine or methadone administered during admission: buprenorphine sublingual tablet; buprenorphine/naloxone sublingual film; buprenorphine/naloxone sublingual tablet; buprenorphine injection; buprenorphine patch; methadone injection; methadone solution concentrate; methadone solution oral; methadone tablet; methadone tablet effervescent; and methadone unknown formulation.

\section{Post-Admission OAT}

Post-admission OAT included a visit to an VHA OTP (counted by VHA stop code) or HCPCS codes (H0033: non-specific oral medication direct observation; J0574: buprenorphine/naloxone 6.1-10 mg; J0575: buprenorphine/naloxone > 10 mg; J0571: oral buprenorphine $1 \mathrm{mg}$; S0109:

methadone oral $5 \mathrm{mg}$ ) or outpatient buprenorphine filled prescriptions (only FDA-approved OAT formularies and routes of administration were included: buprenorphine film buccal; buprenorphine sublingual tablet; buprenorphine/naloxone film sublingual; and buprenorphine/naloxone sublingual tablet).

\section{Specific Admission OAT Scenarios}

Using the definitions in this appendix for pre, admission, and post OAT.

- OAT Continuation: OAT delivery in the 30-days prior to and during admission, but not after.

- OAT Initiation \& Linkage to Care: OAT delivery during admission and post-admission, but not prior to admission.

- OAT Sustained: OAT delivery before admission, during admission, and post-admission.

- OAT Withdrawal: OAT delivery during admission, but not prior to admission and after admission. 


\section{Appendix P: Patient Health \& Utilization Definitions}

\section{In-Hospital Mortality}

In-hospital mortality was constructed using the VHA disposition variables (\#6 and \#7): death with autopsy or death without autopsy during index hospitalization.

\section{Left Against Medical Advice}

Left against medical advice was constructed from VHA disposition variables (\#2 and \#4):

irregular or NBC or While ASIH (Absent Sick in Hospital).

\section{0-Day Death}

30-day death from index hospitalization discharge was captured from the VHA death data sources: died during care provided by the VHA, BIRLS (i.e., national cemetery database), CMS, Social Security Administration, VHA Benefits Division, and FEE Basis Care. This does not include cases that were registered as having a death but health care resources were utilized post-death.

\section{0-Day Hospital Readmission}

30-day hospital readmission was captured when a patient was re-admitted to a VHA facility for acute care within 30 days after the index hospitalization.

\section{0-Day Emergency Department Visit}

30-day emergency department visit was captured when a patient was seen in a VHA Emergency Department (stop code 130) within 30 days after the index hospitalization. 


\section{Appendix Q: Hospital Characteristic Definitions}

\section{Admission Volume}

The number of admissions in that facility with the study cohort population.

\section{Acute OUD Diagnosis Volume}

The percentage of admissions out of all admissions in that facility with an acute OUD diagnosis. As defined in Appendix $M$ as either an OUD-related infection or any OUD diagnosis in the primary or secondary admission diagnosis spot.

\section{Hospital Census Region}

Using the hospital state location hospitals were labeled as existing in one of the four U.S. Geographic Census Regions (U.S. Census Bureau, 2015): Northeast; Midwest; South; and West.

\section{Hospital Size}

Bed counts for "acute care hospitals" (an additional report produced internally at the VHA) included hospitals that had at least 500 acute bed days of care during the year. Bed size was collapsed from four categories ( 1 to 49 beds; 50 to 99 beds; 100 to 199 beds; $\geq 200$ beds) to three for analyses: 1) Small: 1 to 49 beds; 2) Medium: 50 to 99 beds; and 3) Large: $\geq 100$. 


\section{Appendix R: Sensitivity Analysis: OAT Definition}

The models were re-run using a narrower OAT definition for admission delivery; specifically, excluding any non-FDA approved versions of OAT (i.e., injectable formulations of methadone and buprenorphine). There were no differences in OAT quartile cut-offs during data preparation.

Model 1: The Variance Component Model. Meaningful differences were not observed for the narrower OAT definition. The coefficient was the same (and same direction), as were the random effects (ICC: 0.13 vs. 0.13 ).

Model 2: The Intermediate Model with Level 1 Covariates. There were no differences in the intermediate model (Model 2) with regards to covariate direction or statistically significant associations; except for the surgical variable. Which was statistically significant in the model with the broader OAT definition. There was no difference in the ICC between the two models (0.10 vs. 0.10).

Model 3: The Full Model with Level 1 and Level $\mathbf{2}$ Covariates. There were no differences in the final model (Model 3) with regards to covariate direction or statistically significant associations; except for, the surgical variable. Which was statistically significant in the model with the broader OAT definition. The ICCs were the same (0.062).

\begin{tabular}{|c|c|c|c|c|c|c|}
\hline \\
\hline $\begin{array}{l}\text { Output: } \\
\text { Narrow OAT Definition }\end{array}$ & OR & SE & $\mathbf{Z}$ & $\mathbf{P}$ & $95 \% \mathrm{Cl}$ & \\
\hline admit_age & 1.000299 & 0.002539 & 0.12 & 0.9060 & 1.00 & 1.01 \\
\hline male_dum & 1.503346 & 0.2121659 & 2.89 & 0.0040 & 1.14 & 1.98 \\
\hline racecollapse_nonwhite_dum & 0.9459724 & 0.0738042 & -0.71 & 0.4770 & 0.81 & 1.10 \\
\hline racecollapse_unk2_dum & 0.8397542 & 0.149254 & -0.98 & 0.3260 & 0.59 & 1.19 \\
\hline ethnicity_hisp_dum & 0.9842062 & 0.1419715 & -0.11 & 0.9120 & 0.74 & 1.31 \\
\hline ethnicity_unk_dum & 1.201423 & 0.24997 & 0.88 & 0.3780 & 0.80 & 1.81 \\
\hline _loud_dxinf_1 & 2.298961 & 0.1720454 & 11.12 & 0.0000 & 1.99 & 2.66 \\
\hline _lcosud_all_1 & 0.7782597 & 0.0543631 & -3.59 & 0.0000 & 0.68 & 0.89 \\
\hline _Icomentalh_1 & 0.9536874 & 0.0677582 & -0.67 & 0.5050 & 0.83 & 1.10 \\
\hline _lunintent__ 1 & 0.2717729 & 0.0836167 & -4.23 & 0.0000 & 0.15 & 0.50 \\
\hline _Iwd_codes_1 & 1.50842 & 0.206946 & 3 & 0.0030 & 1.15 & 1.97 \\
\hline index_los & 1.043432 & 0.0045775 & 9.69 & 0.0000 & 1.03 & 1.05 \\
\hline _licu_fflag_1 & 0.8893198 & 0.0745036 & -1.4 & 0.1610 & 0.75 & 1.05 \\
\hline _Isurgery_f_1 & 0.766244 & 0.1058284 & -1.93 & 0.0540 & 0.58 & 1.00 \\
\hline admitsource_other_dum & 0.8804784 & 0.1260072 & -0.89 & 0.3740 & 0.67 & 1.17 \\
\hline admitsource_direct_dum & 0.9829147 & 0.0771065 & -0.22 & 0.8260 & 0.84 & 1.15 \\
\hline _lanyopioid_1 & 0.5408422 & 0.0376208 & -8.84 & 0.0000 & 0.47 & 0.62 \\
\hline _lanyadj_co_1 & 1.51515 & 0.1087461 & 5.79 & 0.0000 & 1.32 & 1.74 \\
\hline _lbenzo_1 & 0.9098837 & 0.06681 & -1.29 & 0.1980 & 0.79 & 1.05 \\
\hline _Inaltrexon_1 & 0.3129734 & 0.1215771 & -2.99 & 0.0030 & 0.15 & 0.67 \\
\hline _Ipre_anyoa_1 & 15.04093 & 1.143896 & 35.64 & 0.0000 & 12.96 & 17.46 \\
\hline _Ipre_anyop_1 & 0.482209 & 0.0418063 & -8.41 & 0.0000 & 0.41 & 0.57 \\
\hline Ipre_benzo_1 & 0.9870739 & 0.1243564 & -0.1 & 0.9180 & 0.77 & 1.26 \\
\hline _Ipre_naltr_1 & 0.2692671 & 0.1035082 & -3.41 & 0.0010 & 0.13 & 0.57 \\
\hline _Ipre_gaba_1 & 0.8937247 & 0.0687118 & -1.46 & 0.1440 & 0.77 & 1.04 \\
\hline oud_dxinf_rel_vol_percent & 0.9824978 & 0.0069802 & -2.49 & 0.0130 & 0.97 & 1.00 \\
\hline bed_cat_collapse_med_dum & 1.89009 & 0.3410116 & 3.53 & 0.0000 & 1.33 & 2.69 \\
\hline
\end{tabular}




$\begin{array}{lrrrrrr}\text { bed_cat_collapse_large_dum } & 1.905646 & 0.3767839 & 3.26 & 0.0010 & 1.29 & 2.81 \\ \text { census_reg_mw_dum } & 1.25838 & 0.1970601 & 1.47 & 0.1420 & 0.93 & 1.71 \\ \text { census_reg_ne_dum } & 1.600228 & 0.2682119 & 2.81 & 0.0050 & 1.15 & 2.22 \\ \text { census_reg_w_dum } & 1.626464 & 0.2603606 & 3.04 & 0.0020 & 1.19 & 2.23 \\ \text { admissions } & 0.9989553 & 0.0010102 & -1.03 & 0.3010 & 1.00 & 1.00 \\ \text { _cons } & 0.0599586 & 0.0234484 & -7.2 & 0.0000 & 0.03 & 0.13 \\ \text { fac_study_id: var (cons) } & 0.2176029 & 0.0507659 & - & - & 1.00 & 1.01\end{array}$

\begin{tabular}{|c|c|c|c|c|c|c|}
\hline $\begin{array}{l}\text { Output: } \\
\text { Broader OAT Definition }\end{array}$ & OR & SE & $\mathbf{Z}$ & $\mathbf{P}$ & $95 \% \mathrm{Cl}$ & \\
\hline admit_age & 1.00 & 0.0025329 & 0.17 & 0.86800 & 1.00 & 1.01 \\
\hline male_dum & 1.52 & 0.2151393 & 2.98 & 0.00300 & 1.16 & 2.01 \\
\hline racecollapse_nonwhite_dum & 0.95 & 0.0738871 & -0.65 & 0.51600 & 0.82 & 1.11 \\
\hline racecollapse_unk2_dum & 0.84 & 0.1484017 & -1 & 0.31700 & 0.59 & 1.19 \\
\hline ethnicity_hisp_dum & 1.02 & 0.1444581 & 0.13 & 0.89700 & 0.77 & 1.34 \\
\hline ethnicity_unk_dum & 1.20 & 0.250349 & 0.88 & 0.37700 & 0.80 & 1.81 \\
\hline _loud_dxinf_1 & 2.30 & 0.1719125 & 11.170 & 0.00000 & 1.99 & 2.66 \\
\hline _lcosud_all_1 & 0.77 & 0.0537238 & -3.730 & 0.00000 & 0.67 & 0.88 \\
\hline _Icomentalh_1 & 0.97 & 0.0684419 & -0.49 & 0.62100 & 0.84 & 1.11 \\
\hline _lunintent__1 & 0.29 & 0.0871154 & -4.120 & 0.00000 & 0.16 & 0.52 \\
\hline _Iwd_codes_1 & 1.47 & 0.2013285 & 2.79 & 0.00500 & 1.12 & 1.92 \\
\hline index_los & 1.04 & 0.0045693 & 9.760 & 0.00000 & 1.03 & 1.05 \\
\hline _licu_flag_1 & 0.88 & 0.0732433 & -1.58 & 0.11500 & 0.74 & 1.03 \\
\hline _Isurgery_f_1 & 0.75 & 0.1041471 & -2.06 & 0.04000 & 0.57 & 0.99 \\
\hline admitsource_other_dum & 0.88 & 0.1255941 & -0.92 & 0.36000 & 0.66 & 1.16 \\
\hline admitsource_direct_dum & 0.99 & 0.0773472 & -0.16 & 0.87600 & 0.85 & 1.15 \\
\hline _lanyopioid_1 & 0.53 & 0.0367234 & -9.1700 & 0.00000 & 0.46 & 0.61 \\
\hline _lanyadj_co_1 & 1.52 & 0.1089526 & 5.8600 & 0.00000 & 1.32 & 1.75 \\
\hline _Ibenzo_1 & 0.92 & 0.0672824 & -1.15 & 0.24900 & 0.80 & 1.06 \\
\hline _Inaltrexon_1 & 0.31 & 0.120364 & -3.02 & 0.00300 & 0.14 & 0.66 \\
\hline _Ipre_anyoa_1 & 15.26 & 1.164097 & 35.720 & 0.00000 & 13.14 & 17.72 \\
\hline _Ipre_anyop_1 & 0.49 & 0.0420021 & -8.350 & 0.00000 & 0.41 & 0.58 \\
\hline _Ipre_benzo_1 & 1.03 & 0.1280635 & 0.25 & 0.80400 & 0.81 & 1.32 \\
\hline _Ipre_naltr_1 & 0.26 & 0.1017697 & -3.46 & 0.00100 & 0.12 & 0.56 \\
\hline _Ipre_gaba_1 & 0.89 & 0.0683887 & -1.5 & 0.13300 & 0.77 & 1.04 \\
\hline oud_dxinf_rel_vol_percent & 0.98 & 0.0069608 & -2.7 & 0.00700 & 0.97 & 0.99 \\
\hline bed_cat_collapse_med_dum & 1.90 & 0.3416866 & 3.560000 & 0.00000 & 1.33 & 2.70 \\
\hline bed_cat_collapse_large_dum & 2.04 & 0.4020263 & 3.62 & 0.00000 & 1.39 & 3.00 \\
\hline census_reg_mw_dum & 1.27 & 0.1978938 & 1.5 & 0.13300 & 0.93 & 1.72 \\
\hline census_reg_ne_dum & 1.80 & 0.2990432 & 3.52 & 0.00000 & 1.30 & 2.49 \\
\hline census_reg_w_dum & 1.62 & 0.259615 & 3.03 & 0.00200 & 1.19 & 2.22 \\
\hline admissions & 1.00 & 0.0010067 & -1.47 & 0.14300 & 1.00 & 1.00 \\
\hline _cons & 0.06 & 0.0243274 & -7.11000 & 0.00000 & 0.03 & 0.13 \\
\hline fac_study_id: var (cons) & 0.22 & 0.05 & -- & -- & 0.14 & 0.34 \\
\hline
\end{tabular}

